\title{
A Neurophysiological Interpretation of the Respiratory Act.
}

\author{
By \\ RoBeRT GESELL-Ann Arbor, Michigan ${ }^{1}$.
}

With 174 figures.

Contents.

1. The Nature of the Problem Pages

1. The Nature of the Problem . . . . . . . . . . . . . . . . . . 481

2. Muscular Energy Requirements for Breathing . . . . . . . . . . . . . 482

3. The Basic Mechanisms of Gradation of the Intensity of Respiratory Contractions . 486

4. Central Activity Patterns . . . . . . . . . . . . . . . . . . . 495

5. The Respiratory Center . . . . . . . . . . . . . . . . . . . . 499

6. The Origin of Respiratory Activity Patterns . . . . . . . . . . . . . . . . 502

7. The Electrotonic Theory of Nerve Cell Activity . . . . . . . . . . . . . . . 504

8. The Electrical Requirements of Electrotonic Excitation . . . . . . . . . . . 507

9. The Gradation of the Self Engendered Electrotonic Excitation . . . . . . . . 508

10. The Electrotonic Theory of Synaptic Drive . . . . . . . . . . . . 510

11. After-Discharge . . . . . . . . . . . . . . . . . . . . . . 512

12. The Recurrent Collateral as a Challenge to the Theory of Synaptic Transmission . 517

13. An Electrotonic Theory of Nerve Cell Inhibition. . . . . . . . . . . . . . 520

14. An Hypothetical Structural Basis of Respiratory Activity Patterns . . . . . . . . 525

15. A central Neuro Structural Basis for Recruitment. . . . . . . . . . . . 527

16. Central Reciprocating Interaction of the Respiratory Half-Center . . . . . . . . . 529

17. The Central Excitatory State and the Central Inhibitory State . . . . . . . . 531

18. The Dual Driving Action (Inspiratory and Expiratory) of the Chemoceptor Signals . 534

19. The Double Driving Action of the Nociceptor Signals . . . . . . . . . . . . 536

20. The Reflexogenic Support of the Vagal Proprioceptive Signals . . . . . . . . . . 537

21. The Reflexogenic Support of the GoLGI Signals . . . . . . . . . . . . . 555

22. The Reflexogenic Support of the Muscle Spindles . . . . . . . . . . . . . . . 559

23. A Comparison of the Respiratory Act with Rhythmic Motor Activities of the Spinal Cord 559

24. The Individual Localization of the Inspiratory and Expiratory Centers . . . . . . . 570

25. Inspiratory Tetanus or Apneustic Breathing . . . . . . . . . . . . . . 579

26. The Role of the Spinal Cord . . . . . . . . . . . . . . . . 592

27. The Harmony of Physical and Chemical Drives . . . . . . . . . . . 596

28. The Influence of Chemical Equilibrium Upon Respiratory Reflexes . . . . . . . . 601

29. The Nature of the Chemical Stimulus of Breathing . . . . . . . . . . . 606

30. The Interaction of Central and Peripheral Chemical Control of Breathing. . . . . 622

31. A Comparison of Hypooxic and Hypercapnic Hyperpnea . . . . . . . . . . 633

1 Department of Physiology University of Michigan. Ann Arbor, Michigan. U. S. A. 


\section{Bibliography.}

1. Gasert, R.: Erg. Physiol. 28, 339 (1929).

2. - Amer. J. Physiol. 115, 168 (1936).

3. Brown, R. C., A. K. Atkinson and R. Gesell: Amer. J. Physiol. 126, 447 (1939).

4. Gesele, R.: Amer. J. Physiol. 116, 228 (1936).

5. Adrian, E. D. and D. W. Broxk: J. of Physiol. 66, 81 (1928).

6. - - J. of Physiol. 67, 119 (1929).

7. Bronk, D. W. and L. K. Ferguson: Amer. J. Physiol. 110, 700 (1935).

8. RiJlant, P.: Arch. internat. Physiol. 44, 351 (1937).

9. Atkinson, A. K., R. C. Brown and R. Gesell: Amer. J. Physiol. 129, 303 (1940).

10. Creed, R. S., D. Denny Brown, J. C. Eccles, E. G. T. Liddelle and C. S. SherringTon: Reflex Activity of the Spinal Cord, p. 179. Oxford: Clarendon Press 1932.

11. Cooper, S. and J. C. Eccles: J. of Physiol. 69, 378 (1930).

12. Geselt, R., J. Bricker and C. Magee: Amer. J. Physiol. 117423 (1936).

13. Matthews, B. H. C.: J. of Physiol. 79, 153 (1931).

14. Longet, A.: Arch. gén. Med., IV.s. 13, 374 (1847).

15. Flourens, J. P. M.: C. r. Acad. Sci. Paris 47, 803 (1858).

16. Mislawsky, N.: Zbl. med. Wiss. 23, 465 (1885).

17. LaBorde, J.: C. r. Soc. Biol. Paris, N. s. 2, 620 (1890).

18. Gad, J. and G. Mafinescu: C. r. Acad. Sci. Paris 115, 444 (1892).

19. Arnwein: Arch. f. (Anat. u.) Physiol. 1 (I894).

20. Kohnstamm, O.: Mschr. Psychiatr. (1900).

21. BeCHTEREw, W.: Die Functionen der Nervencentra. Jena 1908.

22. Fintex, K. H.: Arch. of Neur. 26, 754 (1931).

23. PAPEZ, J. W.: J. comp. Neur. 41, 365 (1926).

24. Alien, W. F.: J. comp. Neur. 48, 45I (1927).

25. Heinderson, V. E. and E. H. Craigte: Amer. J. Physiol. 115, 520 (1936).

26. Ramon y Carad: Histologie du système Nervenx. Vol. 1, p. 753. Paris: A. Maloine 1909.

27. PitTs, R. F., H. W. Magoun and S. W. Ratison: Amer. J. Physiol. 126, 673 (1939).

28. Gesell, R., A. K. Atkinson and R. C. Browa: Amer. J. Physiol. 128, 629 (1940).

29. Henges, E. S. and J. E. MeYers: The Problem of Physico-Chemical Periodicity, p. 95. London: Green and Co. 1926.

30. Romandes, G. J.: Jelly-fish, Starfish and Sea-Urchins, p. 323. London: Kegan Paul, Trench and Co. 1885.

31. Carlsov, A. J.: Erg. Physiol. 3, 371 (1909).

32. Adrian, E. D. and F. J. J. BuytendiJk: J. of Physiol. 71, 121 (1931).

33. Rosenthau, J.: Die Atembewegungen und ihre Beziehungen zum Nervus vagus, S. 272. Berlin: August Hirschwald 1862.

34. Marckwacd, M.: The Movements of Respiration, p. 175. Glasgow: Blackie and Son 1888.

35. Hexmans et Bouctianit: Erg. Physiol. 41, 28 (1939).

36. Geselt, R. : Proc. Soc. exper. Biol. a. Med. 24, 263 (1926).

37. - Erg. Physiol. 28, 340 (1929).

38. - Univ. Hosp. Bull. (Michigan) 5, 12 (1939).

39. - Amer. J. Physiol. 126, 500 (1939).

40. - Science (N. Y.) 91, 229 (1940).

41. - Heart, Blood and Circulation (Amer. Assoc. Adv. Sci. Monograph). Lancaster, Pa.: Science Press 1940.

42. LimLte, R. S.: Protoplasmic Action and Nervous Action, p. 417. Chicago: Univ. Press 1923.

43. GARTEN, S.: Z. Biol. 52, 534 (1909).

44. IJILIII, R. S.: Arch. Sei. Biol. 12, 102 (1928).

45. Osterhout, W. J. V. and S. E. Hils: J. gen. Physiol. 13, 459 (1930).

46. Adrian, E. D.: Proc. roy. Soc. Lond. 106 (B), 596 (1930).

47. Holmes: Biochemic. J. 26, 2005 (1932).

48. Chaigte, E. H.: J. comp. Neur. 42, 57 (1926). 
49. Gerard, R. W.: Amer. J. Physiol. 92, 498 (1930).

50. - Physiologic. Rev. 12, 469 (1932).

51. - Proc. Assoc. Res. Nerv. Mental Dis. 18, 316 (1938).

52. Karpers, A., G. C. Hubgr and E. C. Crosby: Comparative Anatomy of the Nervous System of Vertebrates Including Man., Vol. 1, p. 864. New York: Macmillan 1936.

53. Garrex, W. E.: J. gen. Physiol. 3, 41 (1920).

54. - J. gen. Physiol. 3, 49 (1920).

55. Hoagrand: Cold Spring Harbor Symposia on Quantitative Biology, Vol. 4, p. 267. 1936.

56. Bartelemez, G. W.: J. comp. Neur. 25, 87 (1915).

57. LORGNTE DF No, R.: J. of Neurophysiol. 1, 195 (1938).

58. BoDIAN, D.: J. comp. Neur. 68, 117 (1937).

59. Creed, R. S., D. Denny Brown, J. C. Eccles, E. G. T. Liddell and C. S. Sherrington: Reflex activity of the spinal cord, p. 179. Oxford: Clarendon Press 1932.

60. BaRD, P.: MACLmods Physiology in Modern Medicine, 8th ed. St. Louis: C. V. Mosby Comp. 1938.

61. Sherrington, C. S.: Integrative Action of the Nervous System, XVI, p. 4l1. New York: Scribners 1906.

62. Bronk, D. W.: J. of Neurophysiol. 2, 380 (1939).

63. --- and M. G. Larabee: Amer. J. Physiol. 119, 279 (1937).

64. Geseld, R., M. A. Hamilton and C. R. Brassfield: To be published.

65. Gasser, H. S.: J. of Neurophysiol, 2, 361 (1939).

66. Ertanger, J.: J. of Neurophysiol. 2, 370 (1939).

67. LoRHATE DE No, R.: J. of Neurophysiol. 2, 402 (1939).

68. Forbes, A.: J. of Neurophysiol. 2, 465 (1939).

69. Eccless, J. C. and H. E. Hoff: Proc. roy. Soc. Lond. 110 (B), 483 (1932).

70. ErLangER, J. and H. S. GASSER: Electrical signs of nervous activity. (The Eldridge Reeves Johnson Foundation Lectures.) X, p. 221. Philadelphia Penn.; Univ. Press 1937.

71. Creed, R. S., D. Dennx Brown, J. C. Eccues, E. G. T. Liddell and C. S. Sherringron: Reflex activity of the spinal cord, Vol. VII, p. 183. Oxford: Clarendon Press. 1932.

72. Forbes, A.: Physiologic. Rev. 2, 361 (1922).

73. Gasser, H. S.: The control of excitation in the nervous system. Harvey Lectures 169 $\{1936 / 37)$.

74. - and H. Grundfest: Amer. J. Physiol. 117, 113 (1936).

75. Brown, T. G.: Proc. roy. Soc. Lond. 84,308 (1911).

76. - J. of Physiol. 48, 18 (1914).

77. Sherrington, C. S.: Selected writings of (edited by D. Denny-Brown). New York: Hoeber 1940.

78. GeSele, R. and F. White: Amer. J. Physiol. 122, 48 (1938).

79. - and C. A. Moyer: Quart. J. exper. Physiol. 25, 13 (1935).

80. Herive, E. and J. Brever: Die Selbststeuerung der Atmung durch den Nervus vagus. Sitzgsber. Akad. Wiss. Wien, Math.-naturwiss. Kl. 58, (2. Abt.), 909 (1868).

81. Gad, J.: Arch. f. Anat. Physiol. (Physiol. Abt.) Lpz. 1, 1 (1880).

82. Head, H.: J. of Physiol. 10, 279 (1889).

83. Adrian, E. D.; J. of Physiol. 79, 332 (1933).

84. Hirlembrand and Boyd: Amer. J. Physiol. 116, 380 (1936).

85. BoYi, T. E. and C. A. MaAske: J. of Neurophysiol. 12, 533 (1939).

86. Hammotda, M. and W. H. Wilson: J. of Physiol. 74, 81 (1932).

87. - - J. of Physiol. 80, 26 (1933).

88. - - J. of Physiol. 94, 497 (1939).

89. LaRseld, O. and R. S. Dow: Amer. J. Anat. 52, 125 (1933).

90. Hess, W. R.: Die Regulierung der Atmung. Leipzig: Georg Thieme 1931.

91. - Pflügers Arch. 226, 198 (1930).

92. - and O. A.M. Wxss: Pflügers Areh. 287, 761 (1936).

93. WYss, O. A. M.: Pflügers Arch. 242, 215 (1939). 
94. Gestex, R.: In „Livro de Homenagem" aos Professores Alvaro e Miguel Ozorio de Almidid, p. 295. Rio de Janeiro, Brasil. 1939.

95. Hess, W. R.: Sonderabdruck aus den Verhandlungen der Freien Vereinigung schweizerischer Physiologen. Januar 1938.

96. Fulton, F. G. and J. P. SuñER: Amer. J. Physiol. 83, 554 (1928).

97. Worzntak, J. J. and R. Geselt. Amer. J. Physiol. 126, 658 (1939).

98. Magae, C. S., J. W. Brickar and R. Geseld: Amer. J. Physiol. 129, 415 (1940).

99. Fleisch, A.: Erg. Physiol. 36, 249 (1934).

100. - and TrTpod: Pflügers Arch. 241, 676 (1933).

101. Brown, T. G.: Proc. roy. Soc. Lond. 84, 308 (1911).

102. - J. of Physial. 48, 18 (1914).

103. Nager: Quoted from SherRingtons Integrative Action of the Nervous System. 1894.

104. Hamition, M. A. and R. Geseltl: Amer. J. Physiol. 129, 373 (1940).

105. Gessild, R., E. Srefrensen and J. M. Brooknart: Amer. J. Physiol. 120, 105 (1938).

106. Sherrington, C. S. and Sowton: Selected writings of Sir Charles Sherrington (edited by D. Denny-Brown) New York: Hoeber 1940.

107. Wyss, O. A. M.: Pflügers Arch. 124, 524 (1939).

108. Pitts, R. F., H. W. Magoun and S. W. Ranison: Amer. J. Physiol. 126, 689 (1939).

109. - - - Amer. J. Physiol. 127, 654 (1939).

110. Marckwald, M.: Z. Biol. 5, 149 (1887).

111. Brookhakt, J. M.: Amer. J. Physiol. 126, 446 (1939).

112. Furton, J. F.: Ann. Rev. Physiol. 2, 243 (1940).

113. Steliza, G.: J. of Physiol. 93, 10 (1938).

114. Lumsden, T.: J. of Physiol. 57, 153 (1922).

115. - J. of Physiol. 57, 354 (1922).

116. - J. of Physiol. 58, 81 (1923).

117. - J. of Physiol. 58, 111 (1923).

118. - J. of Physiol. 58, 259 (1923).

119. Nicholson, H. C.: Amer. J. Physiol. 115, 402 (1936).

120. - and D. BreziN: Amer. J. Physiol. 118, 441 (1937).

121. - and S. Sobin: Amer. J. Physiol. 128, 766 (1938).

122. SoвIx, S. and H. C. Nrcrotson: Amer. J. Physiol. 124, 491 (1938).

123. Steli.a, G.: J. of Physiol. 93, 263 (1938).

124. MacDowaL, R. J. S.: Quart. J. exper. Physiol. 16, 291 (1926).

125. Asher, L. and F. Ltscher: Z. Biol. 38, 499 (1899).

126. Geseld, R. and C. Moyer: Quart. J. exper. Physiol. 24, 315 (1935).

127. - - Quart. J. exper. Physiol. 24, 331 (1935).

128. Harrison, T. R., J. A. Calhoun, G. E. Cullin, W. E. Wilzins and C. Pilcher: J. elin. Invest. 11, 133 (1932).

129. - W. G. Harrison, Jr. and J. P. Marsh: Amer. J. Physiol. 100, 417 (1932).

130. - R. Ashman and R. M. Lanson: Arch. int. Med. 49, 151 (1932).

13I. - W. G. Harrison, J. A. CalHoun and J. P. Marsh: Arch. int. Med. 50, 690 (1932).

132. - J. A. CalHoun and W. G. Harrison, Jr.: Arch. int. Med. 53, 911 (1934).

133. WiNder, C.: Amer. J. Physiol. 118, 379 (1937).

134. Kocr, E.: Die Reflektorische Selbststeuerung des Kreislaufes. Dresden u. Leipzig 1931.

135. Lrm, R. K. S. and F. Y. Hst: Chinese J. Physiol. 5, 29 (1931).

136. SchNeXeR, K.: Pflügers Arch. 225, 176 (1934).

137. Бснмiот, C. F.: Amer. J. Physiol. 102, 94 (1932).

138. Brown: Quart. J. exper. Physiol. 2, 243 (1909).

139. KAYA and E. STaringe: J. of Physiol. 39, 346 (1909).

140. Glazer, W.: Amer. J. Physiol. 88, 562 (1929).

141. Winkler, A. W.: Amer. J. Physiol. 89, 243 (1929).

142. - Amer. J. Physiol. 94, 224 (1930).

143. GAY, H.: Amer. J. Physiol. 95, 519 (1930). 
144. Meyerhof, O.: Pflügers Arch. 188, 114 (1921).

145. Hrul, A. V.: Physiologic. Rev, 2, 310 (1922).

146. Gesell, R.: Physiologic. Rev. 5, 551 (1925).

147. - Ann. Rev. Physiol. 1, 185 (1939).

148. Winterstein, H.: Pflügers Arch. 138, 167 (1911).

149. Heymans, C., J. J. Botckalert and L. Dautrebande: Arch. internat. Pharmacodynamie $39,400(1930)$.

150. Gesell, R., H. Krueger, G. Gorham and T. Bernteal: Amer. J. Physiol. 94, 300 (1930).

151. - - H. Nicholson, C. Brassfield and M. Pelecovich: Amer. J. Physiol. 100, 202 (1932).

152. Bernthal, T.: Amer. J. Pbygiol. 121, 1 (1938).

153. Brassfield, C. and V. Behrmann: Amer. J. Physiol. 122, 21 (1938).

154. LUNDSGAARD: Biochem. Z. 217, 162 (1930).

155. - Biochem. Z. 220, 1 (1930).

156. - Biochem. Z. 220, 8 (1930).

157. - Biochem. Z. 227, 51 (1930).

158. Henderson, Y. and L. A. Greenserg: Amer. J. Physiol. 107, 37 (1934).

159. Winder, C.: Amer. J. Physiol. 118, 389 (1937).

160. - T. Bernthal and W. F. Weeks: Amer. J. Physiol. 124, 238 (1938).

161. Bernthal, T. and W. F. Weezs: Amer. J. Physiol. 127, 94 (1939).

162. Euler, U. S. von, G. LluJestrand and Y. Zotterman: Skand. Arch. Physiol. (Berl. u. Lpz.) 83, 132 (1939).

163. Gelleorn, E. and E. W. Lambert: The vasomotor system in anoxia and asphyxia. Urbana: Univ. Illionois Press 1939.

164. Difr, D. B., J. H. Talbott and W. V. Consolario: J. of biol. Chem. 118, 649 (1937).

165. Cromer, S. P. and A. C. Ivy: Proc. Soc. exper. Biol. a. Med. 28, 565 (1931).

166. MoGinty, D. A. and R. Geselle: Amer. J. Physiol. 75, 70 (1925).

167. Dadtrebande, L.: Proc. 16. internat. Physiol. Congr. Zürich 1938. Rio de Janeiro, Brasil. 1939.

168. Dattrebande: Livro de Homenagem aos Professores Alvaro e Miguel, Ozorio de Almeida 1939.

169. Samaan, A. and G. Stellia: J. of Physiol. 85, 309 (1935).

170. Сомroe, J. and C. F. Sснмidт: Amer. J. Physiol. 121, 75 (1938).

171. Schmidt, C. F. and J. H. Comroe: Physiologic. Rev. 20, 115 (1940).

172. Johrson, R. E., W. H. Forbes, D. B. DhL and L. J. Henderson: Ann. Rev. Physiol. 2, 21 (1940).

173. Geseld, R., J. Lapides and M. Levin: Amer. J. Physiol. 130, 155 (1940).

174. Rice, H. V.: Amer. J. Physiol. 124, 535 (1938).

\section{The Nature of the Problem.}

At the time of our last review of respiration in „,Die Ergebnisse der Physiologie" [GeSELx (1)] interest in the chemical control of breathing was confined entirely to the center. But since then the discovery by Heymans of the peripheral chemical control of respiration by the carotid and aortic bodies has changed the outlook on the chemical control of breathing in most important ways. It became desirable to determine not only the relative importance of the central and peripheral chemical control of breathing but also to learn the relative sensitivity of the central and peripheral mechanisms to oxygen lack and carbon dioxide excess. The infinitely greater sensitivity of the chemoceptors to oxygen lack and the relatively unimportance of the chemoceptors 
in hypercapnia were most perplexing questions. The confusion which these findings produced seems now to be approaching a solution for an interaction of the peripheral and central chemical controls gives promise of an explanation as simple and direct as before. There are many indications that acidity can again be considered the main factor of chemical control.

Never before have we been in so favorable a position to attempt a fundamental understanding of the forces which impel the respiratory muscles to ventilate the lungs in proportion to the oxidative needs of the body for advances in neuro-muscular physiology have paved the way. I refer especially to the recent developments in our information on the gradation of muscular contraction, because a fundamental solution of respiratory control must spring from a basic knowledge of the activity of the respiratory muscles. Only after the patterns of activity are adequately revealed can the central integrating forces producing these end activities be definitely established. This in itself means a new treatment of the subject of respiratory control in terms of the fundamentals of neurophysiology. But in addition our own particular treatment must be unique for we have been compelled to abandon the principles of "synaptic transmission" and nerve cell activity upon which older concepts of neurophysiology have been built. Our review thus serves a double purposethat of explaining the adjustment of the respiratory act to the respiratory requirements of the body on a fundamental basis and that of offering new outlooks on the problem of nervous integration in general.

\section{Muscular energy Requirements for Breathing.}

The specific needs of muscular energy for the individual phases of breathing are well recognized in the generally accepted view that active respiration is primarily an inspiratory phenomenon. With the advent of suitable electrical methods the passive nature of eupneic breathing, readily observed in a qualitative way by visual inspection or manual palpation, has now been verified in a more quantitative way [GESELL (2)]. Systematic registration of action potentials of respiratory muscles reveals the greatest variations in expiratory activity. Total absence of expiratory contractions is relatively uncommon. Slight activity on the other hand is very common. Great activity is relatively rare. Thus the general conclusions of the older workers that inspiratory contractions supply the major energy for pulmonary ventilation are confirmed. Only when ventilation is increased above normal are the expiratory muscles thought to play a more important role. Reasonable as this view may seem, electrical methods show the dangers of its general application, for during hypooxic hyperpnea expiratory contractions may actually diminish and withdraw their support completely as ventilation increases [Brown, Atrinson and Geselu (3)]. (See figures 170, 171 and 172.) 
The exclusive use of the inspiratory muscles during graded hyperpnea is a most interesting observation, for it emphasizes the high degree of coordination of the use of the active and passive forces of breathing. As increasing inspirations increasingly distort the lungs and torso, increasing muscular energy must be provided if the inspiratory act is to continue, but in that very adjustment the energy for expiration is also automatically provided. Great exhalations thus have great stores of potential energy at their disposal at the moment the inspiratory muscles relax, thus an extremely simple mechanism allows both deep and rapid breathing essential to high pulmonary ventilation under variable conditions.

The course of inspiratory contraction and relaxation accordingly assume great interest for it determines the rate of movement of air in and out of the lungs (see fig. 1). The records indicate a slow and even filling of the lungs by the uniform downward gradient of the tidal air tracing, and by the relatively small and uniform negative tracheal pressure changes. This even movement of air is accomplished by a gradually increasing strength of contraction of the inspiratory muscles reflected in the progressively rising shadow of the electrogram of the diaphragm.

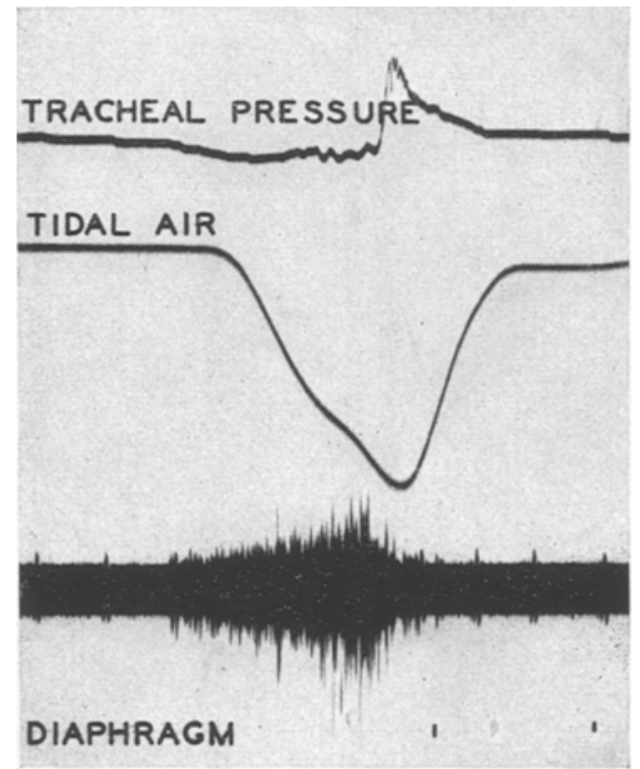

Fig. 1. A record of the action potentials of the diaphragm (slowly augmenting inspiratory fussillade) accompanied by tracheal pressure and tidal air tracings. Downstroke of the tracheal and tidal air tracings correspond to inspiration. To eorrectfor the lag of the spirometer, place the trough of the tidal air tracing immediately above the highest point of the electrioal record. [Brown, ATKINson and Geiseld: Amer. J. Physiol. 126, 447 (1939)].

Expiration follows an entirely different course. The tidal air record rises abruptly, the sudden gush of air produces the momentary expiratory puff in the tracheal pressure record which subsides as the lungs collapse. This rapid powerful expiration, which in this particular instance is purely passive, is produced by the sudden relaxation of the inspiratory muscles and sudden liberation of the potential energy stored in the lungs and torso. The speed of relaxation of the diaphragm is evident in the electrical record. It should be added that as a rule the configuration of the tidal air and tracheal pressure record, such as seen in fig. 1, is little affected by active expiratory contractions unless they be very powerful, indicating once more that expiration is, as a rule, mainly a passive phenomenon. (It is desirable to recognize the universal sluggish response of the spirometer as compared with the tracheal pressure 
record so that the necessary correction may be made on inspection of the records which are to follow.)

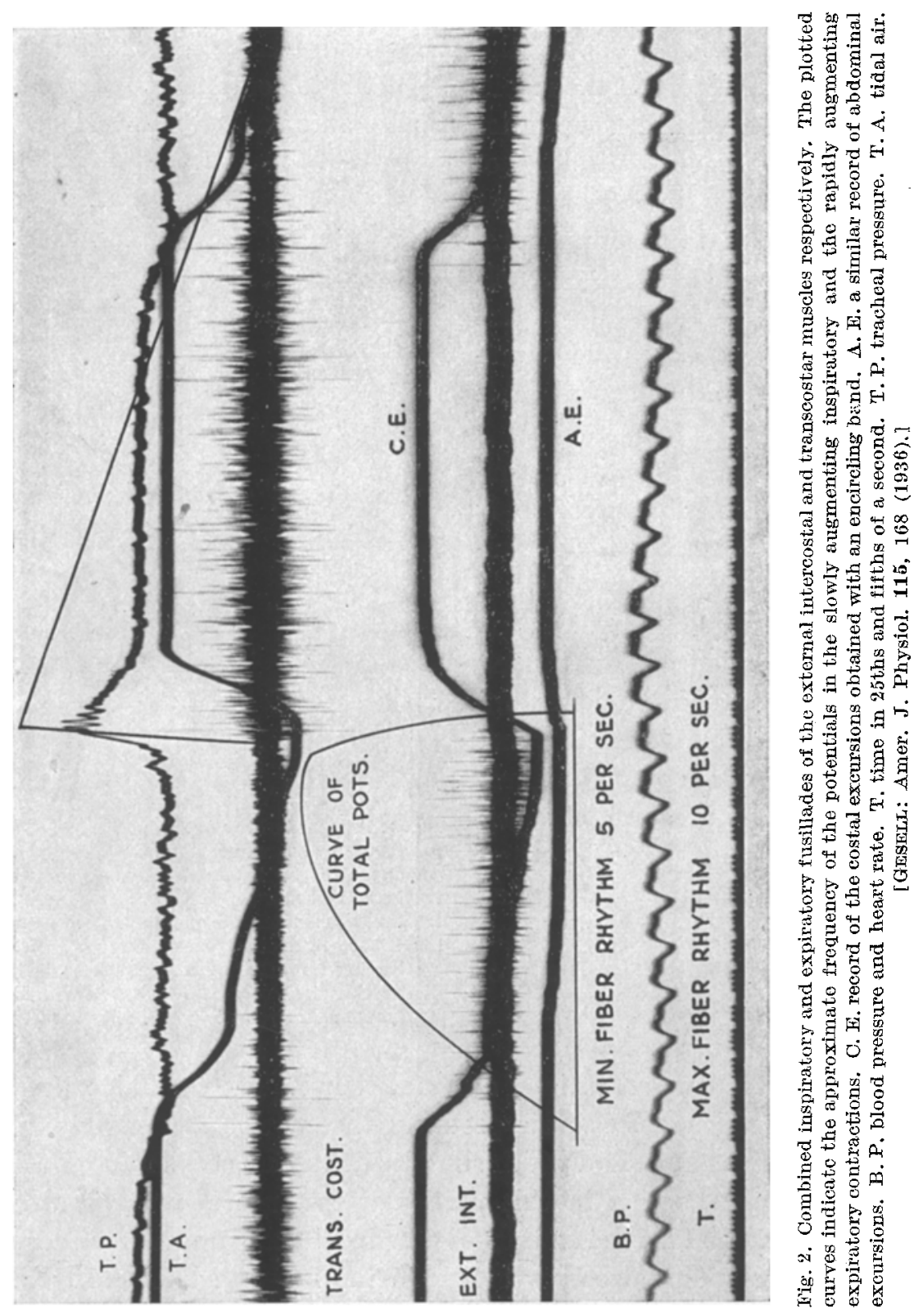

When active expiratory contractions do occur they conform as a rule to one of two common types. They may start with a burst of force at the beginning of the expiratory phase, and decline gradually as illustrated in the contraction of the transcostar muscle in fig. 2. This type of contraction we have referred to as the suddenly augmenting contraction to contrast it with the slowly 
augmenting type of contraction found almost invariably in the inspiratory muscles. The plotted curves, representing the approximate frequency of the
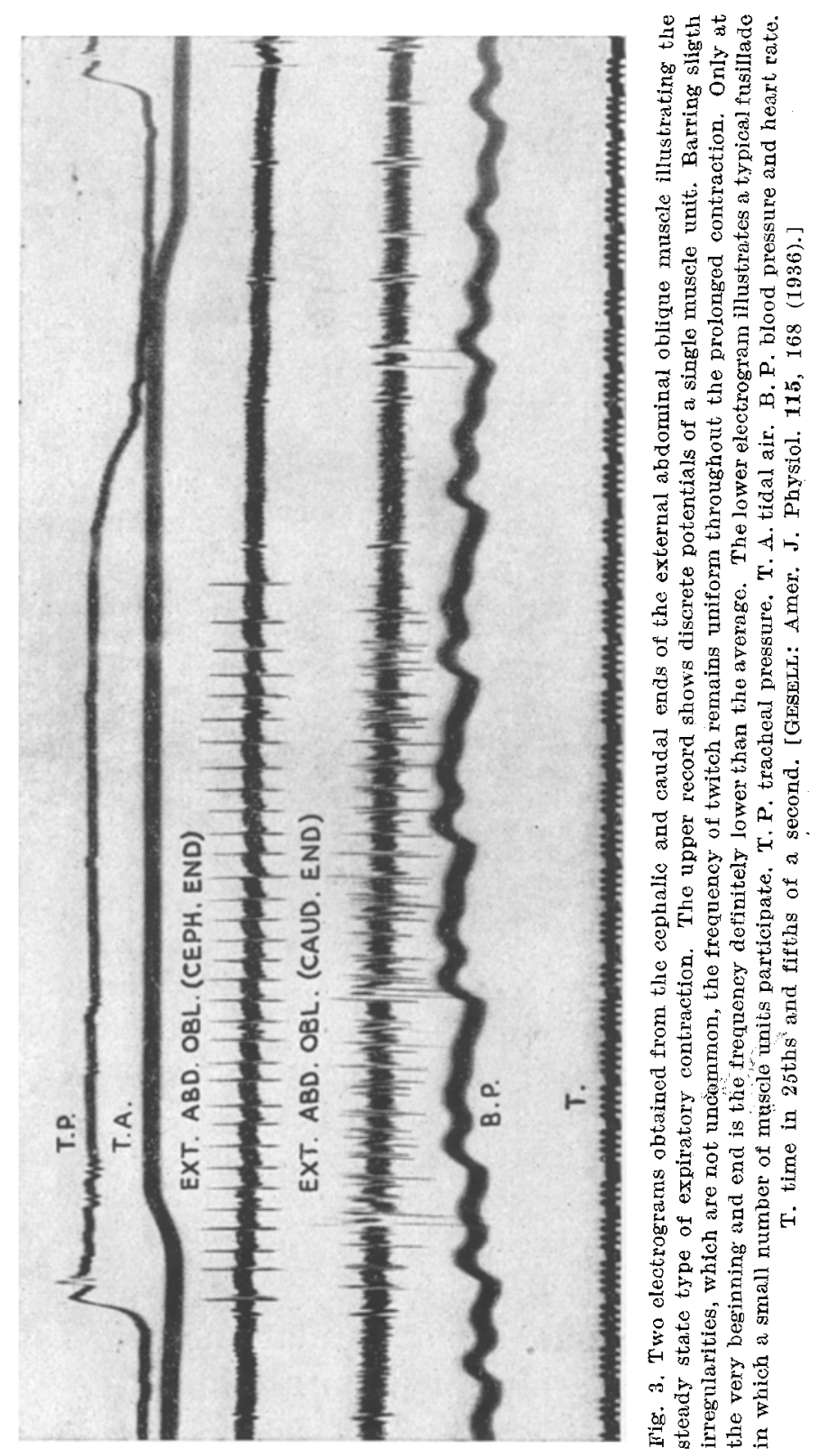

potentials in the inspiratory and expiratory contractions serve to emphasize the contrast. The expiratory contraction corresponds to Sherrington's d'emblee type. As was previously mentioned, fig. 2 shows that these contractions have little influence upon the contour of the tracheal pressure and tidal air records. If the expiratory contractions are to be effective they must 
be very rapid indeed, so as not to fall behind the sudden and purely passive recession of the lungs.

The second type of expiratory contraction, illustrated in fig. 3, was designated as steady state contraction. These contractions are relatively weak and may begin early or late in the expiratory phase, often after the lungs are

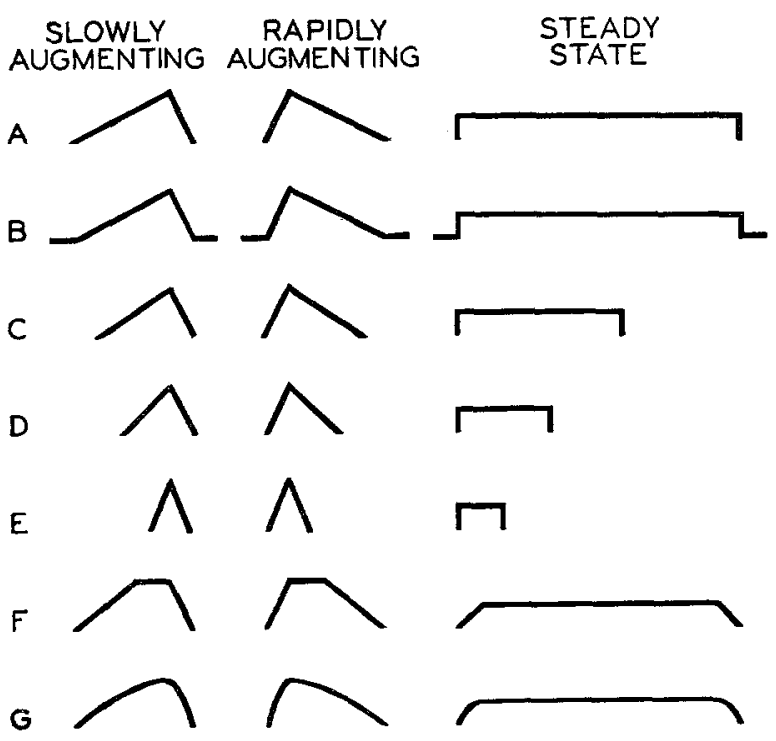

Fig. 4. Schematic representation of the three primary patterns of activity of respiratory muscles. The variations which are shown arise primarily from differences and changes in the frequency of breathing and from the differences in the velocity of development of contraction and relaxation. This schema, serves to illustrate the primary patterns of activity and their variations inthe central respiratory nervous mechanism as well. [Gesell, Magee and Bricker: Amer. J. Physiol. 128, 615 (1940).]

present the three primary types of respiratory contractions. Changes in frequency of breathing and slight variations in the manner of acceleration of muscle fiber discharge and recruitment of new units to be described in the following section must change the appearance of the electrograms. Fig. 4 shows variations frequently met.

\section{The Basic Mechanisms of Gradation of the Intensity of Respiratory Contractions.}

The admirable adjustment of inspiratory contractions to the changing mechanical condition of each inspiratory act is one of the outstanding phenomena of respiration, no doubt the product of a prolonged evolutionary development. The means by which this progressively increasing flow of energy is released to meet the increasing resistance of distortion concerns us now. Thanks to the recent studies on muscular contraction initiated by Adrian and Bronk 
(5 and 6 ) this question is now partially answered. They registered the action potentials of individual units of several motor nerves and muscles and demonstrated that frequency of muscle twitch and number of active muscle units, or recruitment, are the factors determining the strength of muscular contraction. According to these workers the relative importance of variations in frequency of twitch and in number of active units participating in contractions differs with the muscle. For example, in their studies on the single fibers of the phrenic nerve of the rabbit they conclude that the impulses in each nerve fiber recur at a frequency of about 20-30 a second throughout the period of a normal inspiration and "when forcible inspirations are produced by clamping the air tubes to the trachea the frequency of the impulses rises to $50-80$ a second, the highest recorded frequency being 112". "They find that "with low frequency of discharge, as in normal breathing the different nerve fibers do not work in unison, though there is some tendency towards grouping of the discharge; and

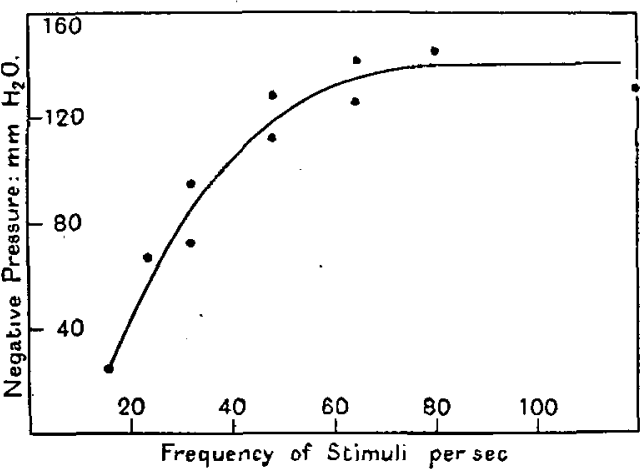

Fig. 5. A plotted curve showing the suction produced in the trachea of the rabbit by stimulation of the phrenic nerve at increasing frequency. [Adrian and Bronk: J. Physiol. 66, 81 (1928).] with high frequencies (in forcible breathing) there is much greater synchronisation, and the discharge consists of successive volleys from the majority of the nerve cells". They consequently believe that "The grading of the contraction in the diaphragm appears to depend mainly on the changes in frequency of the impulse discharge in each nerve fiber. At low frequencies each group of muscle fibers will give an incomplete tetanus but as the groups are not working in unison, the whole muscle will contract smoothly. When the phrenic is stimulated artificially by a series of shocks an increase in frequency from 25 to 60 a sec. doubles the suction produced by the diaphragm". They believe that "variations in frequency of discharge between the limits of 20-80 a second are excellently adapted for producing contractions of graded intensity without bringing fresh muscle fibers into play". Fig. 5 illustrates the increasing suction produced by the diaphragm artificially stimulated at varying frequencies through the phrenic nerve. As support of their contention they present the following statement.

"In the experiments on the rabbit we have looked for evidence of the accession of fresh fibres as the contraction develops by comparing the duration of the single fibre discharge with that of the whole nerve. If more and more fibres come into play during the course of inspiration, the discharge of the whole nerve would usually outlast that of the single fibre, for it is unlikely that the fibre we isolate would always turn out to be one which comes into action at the beginning of inspiration. In general, however, we have found no clear evidence of the single fibre discharge being much shorter than that of the whole nerve." 
In their studies on the frequency of discharge in reflex and voluntary contractions they found in the flexion reflex that "the grading of the contraction appears to be due mainly to this change in frequency, for there is little evidence of changes in the number of neurons in action". In the extension reflexes they found "that many fresh neurones come into play as the contraction develops". Bronk and Ferauson (7) reporting on the internal intercostal muscles conclude that "variations in depth of intercostal respiration are a result of variations in the frequency of discharge from the individual nerve cells, the duration of their discharge and the number of nerve cells in
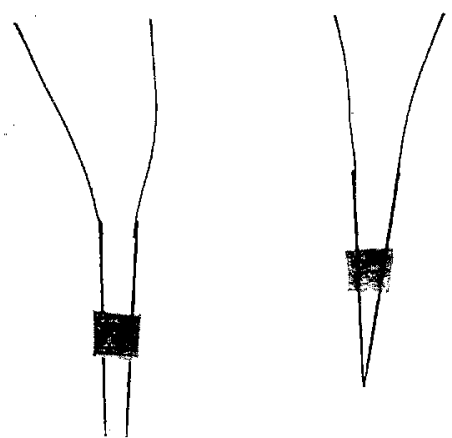

Fig. 6. Floating electrodes employed for the study of action potentials of the respiratory muscles. Fine needles, insulated with shellac and bared only at the very tip, are cemented on tiny flakes of balsa wood. The lead-off wires are phosphor-bronze. A wide separation of leads is suitable for the registration of fusillades. Closerapproximation is moresnitable for the registration of discrete potentials of a single muscle unit. [GEsELL: Amer. J. Physiol. 115, 168 (1936).] action". A really confusing issue is why the gradation of contraction of inspiratory muscles should differ in such important details. Why should the relatively unimportant intercostal muscles deliver a nicely graded contraction suitable for overcoming increasing resistance when the more important diaphragm delivers energy in an explosive block twitching at the very outset with a maximum frequency and maximum number of active units? RiJlant (8) is also of the opinion that the contractions of the diaphragm are of the d'emblee type in the cat, rabbit and dog.

Because a knowledge of the activity patterns of the respiratory muscles seemed to be the first essential to a logical approach of the solution of the problem of the integration of the respiratory act we have studied it in some detail. By simply exposing the main inspiratory muscles and listening to the potentials as the electrodes are moved indiscriminately from one point to another a great deal can be learned of the nature of inspiratory contraction. For that purpose we have employed floating electrodes which consist of two needles, of the finest calibre purchasable, mounted in the manner illustrated in fig. 6 . They rise and fall with the surface of the muscle without the appearance of interfering electrical artefacts. Discrete potentials of single muscle units are readily led off, if the points are closely approximated and carefully placed along the axis of the muscle fibres. When the latter method is used the number of sounds, that is individual twitches, one hears during a single inspiration will vary. With the electrodes in one location there may be as many as 20 . or 30 twitches per inspiration. At another point there may be only one. Such a single twitch always occurs at the close of or near the end of inspiration. When a longer series of twitches occur they begin early in the phase of inspiration. Patient exploration will invariably reveal all intervening variations. 
In those fibers which contract sufficiently long, an acceleration is clearly audible during each inspiration. These results are obtained on the diaphragm as well as on all other inspiratory muscles and, therefore, differ significantly from those of Adrian and Bronk (5).

Fig. 7 is a typical example of the changes in twitch frequency during a single inspiration [Atkinson, Brown and Geseld (9)]. Frequency increases gradually up to the middle or end of inspiration, depending somewhat upon the duration of contraction, and falls to zero at the onset of expiration. Plotted on the ordinates against time on the abscisses, frequency commonly presents a curve of triangular appearance. When the electrodes are more widely separated, a composite record of the activity of many fibers is obtained, and like the frequency curve, the serrated shadow, as is illustrated in fig. 1, presents the same slowly augmenting contour.

The causes of the slowly rising shadow of the electrogram of fig. 1 are traceable to the phenomena of acceleration of frequency of twitch of the individual units and of recruitment of new active units which result in an increasing coincidence of poten-

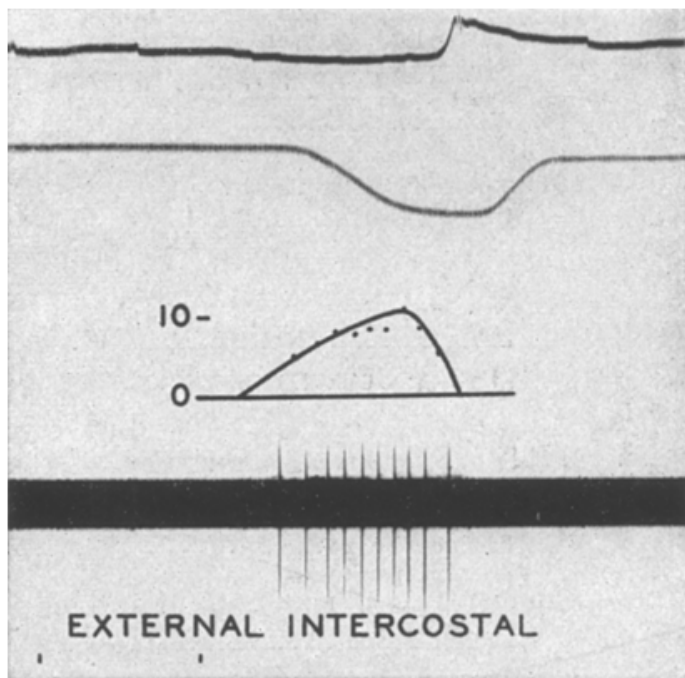

Fig. 7. Disorete inspiratory action potentials of the ex temal intercostal muscle of the dog, showing the slowly augmenting frequency pattern of activity. Frequency per second is plotted immediately above the electrogram. The striking point is the low rate of twiteh. The upper eurves are of tracheal pressure and tidal air. Correction must be made for the lag of the spirometer. [ATKINson, BrowN and GEseld: Amer. J. Physiol. 129, 303 (1940).] tials, such as is illustrated in the schematic inspiratory fusillades of fig. 8. Twenty-nine fiber units were arbitrarily chosen as participating in the eupneic fusillades on the left. Fiber unit \# 1, at the bottom of the schema, opens the contraction and goes through its normal course of acceleration. Fiber \# 2 follows very shortly on \# 1 and Fiber \#3 on \#2, etc. Each fiber begins at its minimum frequency and accelerates up to the end of inspiration (in this schema). The fiber units recruited late in inspiration, therefore, never reach the maximum high frequency of twitch of fiber unit \# 1. This is more clearly seen in the sample discharges of units $1,7,14,21$, and 28 in the skeleton fusillade.

By passing the vertical edge of a card along the fusillade, coincidence of potentials will be seen to increase from left to right, as more and more fibers are added to the contraction and as the frequency of twitch increases. One potential thus adds to the other and raises the level of the electrical record as 
illustrated in the curve of coincidence of fiber activity below. Since each muscle fiber twitch adds its quota of mechanical energy to that of other simultaneously contracting fibers, as it adds its coincident potentials, the rising shadow of a muscle fusillade becomes an index of the strength of muscular contraction. This will explain the automatic increase of contraction occurring during any single inspiration.

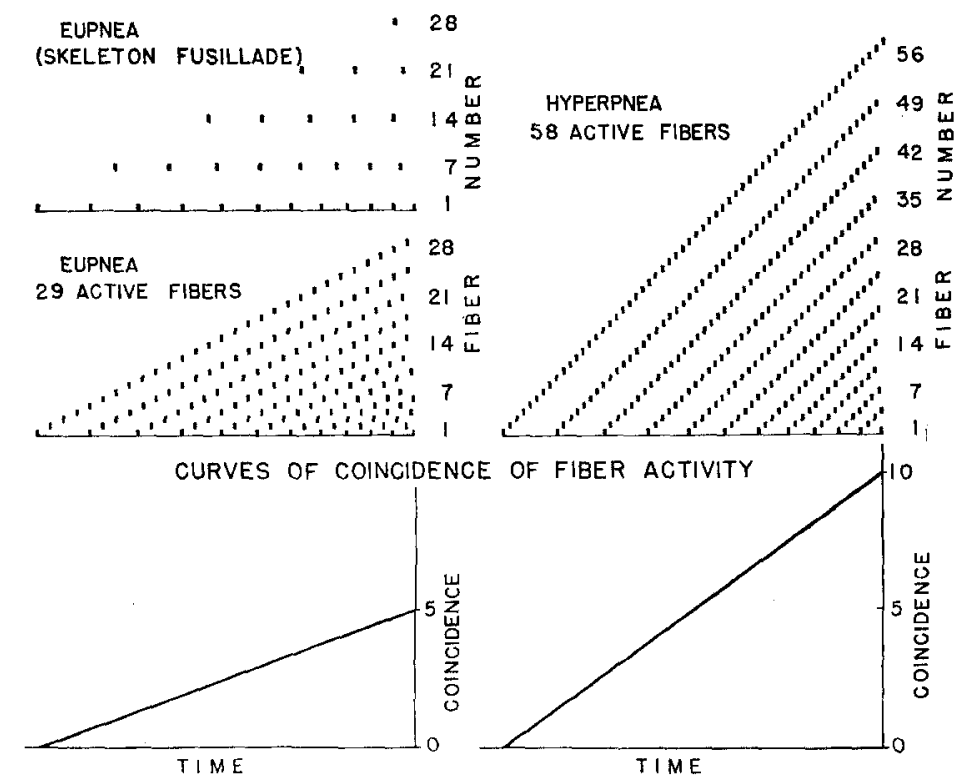

Fig. 8. Schematic inspiratory fusillades illustrating the phenomena of increasing frequency of twitch, of recruitment and of coincidence of muscle unit activity. The left half of the figure is a representation of eupnea and the right half of hyperpnea. Inspiration progresses from left to right. As this happens one active muscle unit after another is added to the contraction thus illustrating the orderly progress of recruitment. The active muscle units are numbered at the right of each fusillade. Each muscle unit increases its frequency of twitch as inspiration waxes. This is more readily seen in the skeleton fusillade. Passing a vertical edge along the fusillade, from left to right, establishes the degree of coincident activity of muscle units which is plotted in the curve below. Doubling the number of active nnits doubles the coincidence of activity and thereby increases the strength of muscular contraction. [ATKINSON, BROWN and GESELL: Amer. J. Physiol. 129, 303 (1940).]

But what are the mechanisms responsible for the progressive increase of breathing which occurs from one inspiration to another during a progressive $\mathrm{O}_{2}$ lack or $\mathrm{CO}_{2}$ excess? They are precisely the same. As the chemical drive increases, more and more fibers are involved with each succeeding breath. For example, during the progressive anoxemia illustrated in fig. 9, muscle fiber \# 1 begins with 7 twitches in respiration \# 1 . In breath 2 the number of twitches has increased to 10. In breaths $3,4,5$ and 6 the twitches have reached a maximum steady number of 12 . Fiber $\# 2$ begins with but a single twitch at the end of inspiration 2 . In breaths 3,4 and 5 it twitches 3,4 and 6 times respectively, and in breath 6 there are 11 twitches in all.

The schematic composite fusillades in figure 8 show in greater detail how eupnea is thus transformed into hypernea. First of all, it will be noted that 
twenty-nine additional fiber units have been added to the eupneic contraction on the right to conform to an hypothetically increased chemical stimulation. But more than that, the initially active fibers have been pressed into greater service. Fibers $7,14,21$ and 28 instead of contracting $8,5,3$ and 1 times are now twitching 10,8,6 and 5 times per inspiration and the newly acquired fibers, numbers 30 to 58 are topping off the inspiratory act. Not only are the
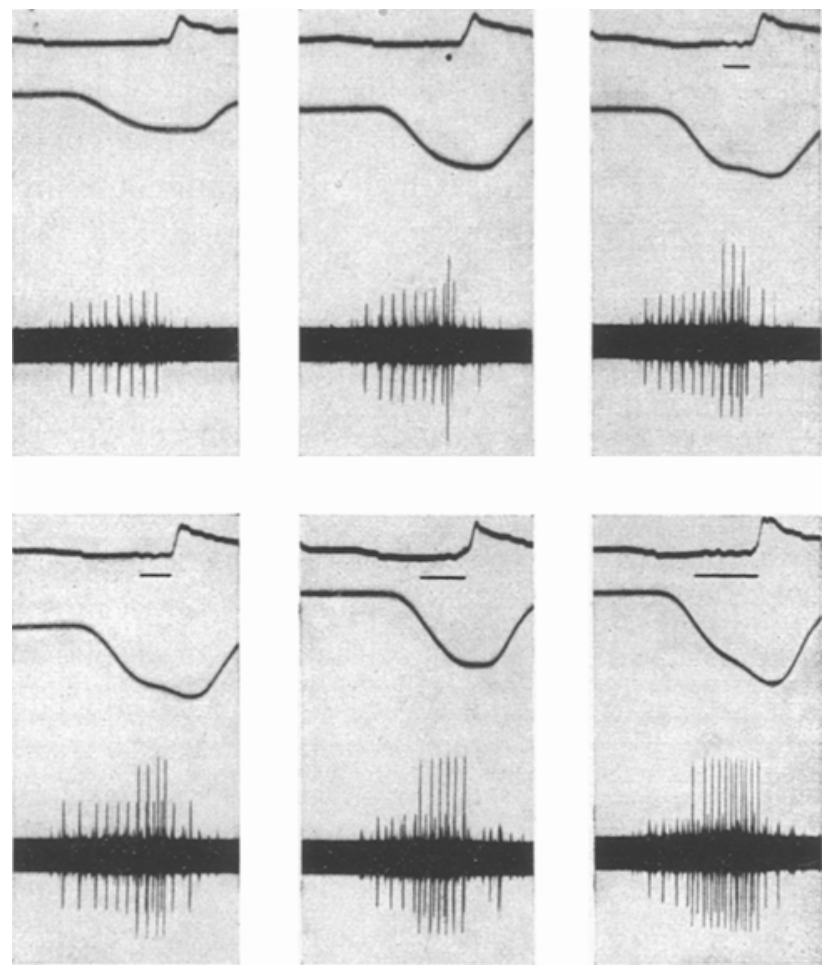

Fig. 9. Inspiratory action potentials of the external intercostal muscle of the dog showing the phenomena of acceleration of twitch of the individual muscle units and the recruitment of new active units during an increasing chemical stimulation produced by rebreathing a small volume of air. The individual activity changes of two muscle units are readily followed. As stimulation increases the duration of activity, the number and frequency of twitches increases for both units. For further analysis see text. [ATKINson, Brown and Geseli: Amer. J. Physiol. 129, 303 (1940).]

older fibers contributing a greater number of twitches but they begin to contract earlier in the phase of inspiration. As a matter of fact, the moment of onset of activity determines importantly the number of times a muscle fiber will twitch.

This advancement of initial activity of each muscle unit towards the beginning of inspiration is shown in fig. 10 in which the actual action potentials of successive respirations during a progressive rebreathing hyperpnea were transposed under a single respiratory schema. Unit \# 1 when first recorded began contracting in the middle of inspiration but as hyperpnea advanced it contracted earlier and earlier as shown by the displacement of the curve to 


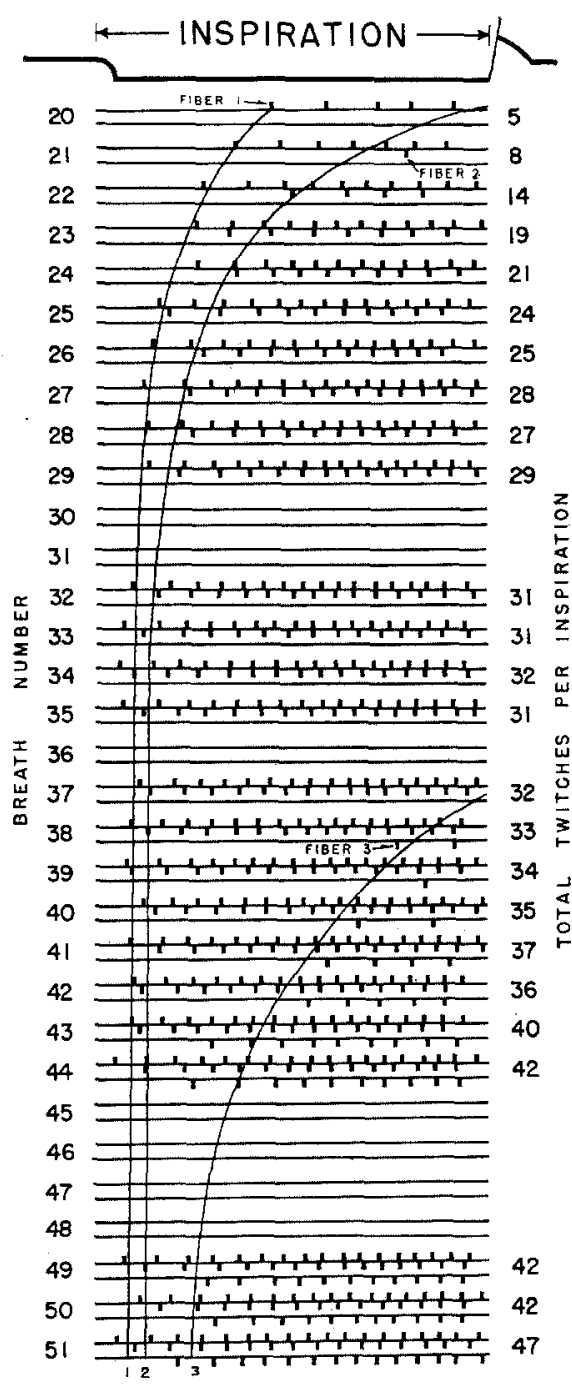

Fig. 10. A serial transposition of electrical records of three muscle units during progressive chemical hyperpnea under a single respiratory schema illustrating primarily the phenomenon of "inspiratory encroachment" of muscle activity. The initial twitches of units 1,2 and 3 connected by curves 1,2 and 3 show that each of the respective units begin to contract earlier in inspiration as the chemical drive increases. The phenomena of recruitment, increasing frequency and increasing coincident activity are illustrated as well. [AKTINSON, BROWN and Grsels: Amer. J. Physiol. 129, 303 (1940). 1 cies of 80 or more in the rabbit. But in the few experiments performed upon the rabbit we too found high frequencies of twitch. Bearing in mind the high frequency of breathing and general agility of the rabbit it is interesting the left. The middle curve shows the inspiratory encroachment of unit \#2 and the lower right hand curve of unit \#3.

It is readily seen that the total number of twitches per inspiration increases for each unit as rebreathing continues. The course of this increase is better shown in fig. 11 in which the total number of twitches for these same fibres is plotted on the ordinates against time or breath number after the beginning of rebreathing on the abscissas. The degree of respiratory stimulation may be assumed to run parallel with the curve of tidal air. As each fiber unit comes into activity the twitch number increases, rapidly at first, then decidedly more slowly, and ends with a tendency to strike a relatively uniform level as compared with the more rapidly rising curve of tidal air. This happens for each muscle unit regardless of the stage of respiratory stimulation at which activity first begins. It is therefore, concluded that there is little agreement between tidal air and total twitch number of any single muscle unit.

Maximum twitch frequency changes in much the same way (see fig. 12). By maximum twitch frequency is meant the highest frequency which a single muscle unit attains during inspiration. This increases rapidly at first with a steadily increasing chemical stimulation, then more slowly, and finally tends to strike a uniform level. Never have we seen a high maximum frequency of twitch in the dog. It ranged between 10 and 20 in the intercostals and 15 and 30 in the diaphragm. In this respect our results might seem to differ also from those of ADRIAN and BRoNK (5) in which they report frequen- 
to speculate whether motor integrations of high tempo do not require a high level of frequency activity of the integrating units.

Since the maximum frequency of twitch of any single unit fails to increase as the strength of contraction of the muscle as a whole continues to increase with increasing chemical stimulation, twitch frequency cannot be the prime factor controlling the strength or depth of inspiratory contraction. This stands in marked contrast to the views of Adrian and Bronk (5). The prime deciding factor is undoubtedly the sum total of muscle fiber twitches occurring during a contraction. When this is plotted for the three muscle units under consideration against intensity of chemical stimulation the curve runs parallel with that of tidal air. Thus each fiber twitch, like each horse added to a team, adds its quota of mechanical energy. This we believe constitutes an important diversion from prevailing views on the gradation of diaphragmatic contractions as set forth by Adrian and Bronk (5). Acceptance of the figures of Sherrington (10), Ecches and Cooper (11), Adrian and Bronk (5), that tetanic contraction may quadruple the

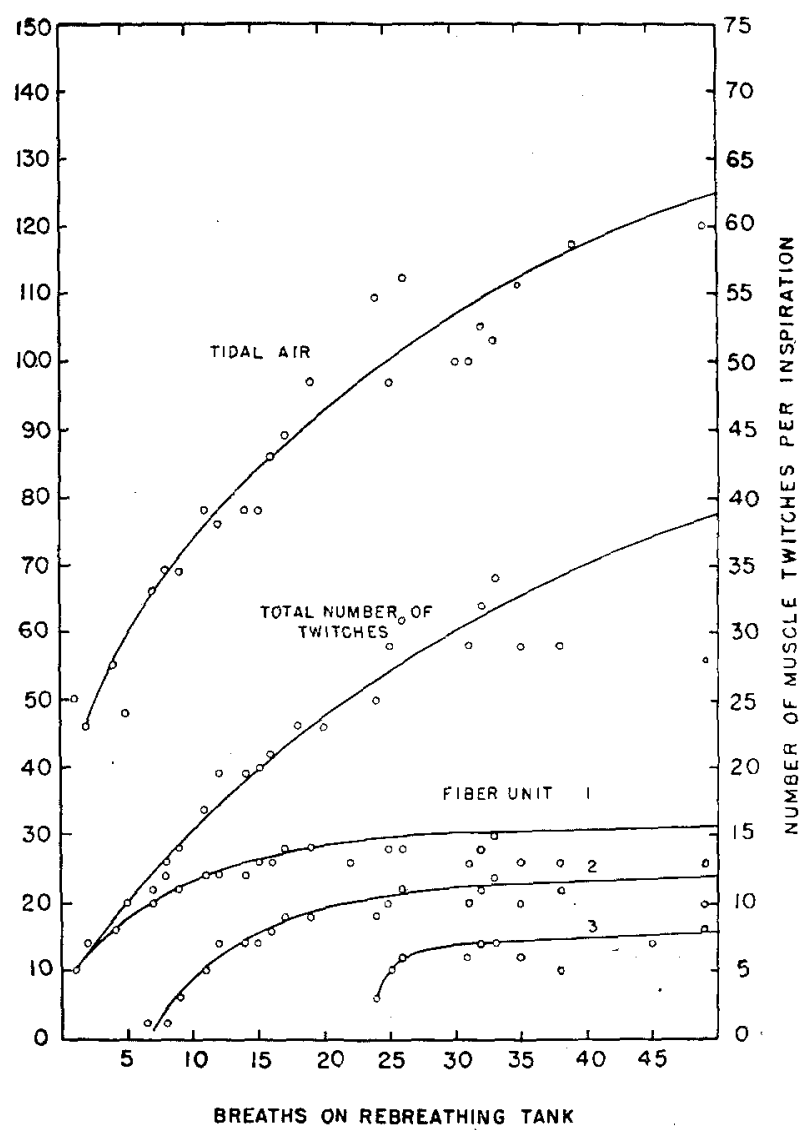

Fig. 11. The three lower curves show the relation of the number of twitches per muscle unit on the ordinates, to the intensity of chemical stimulation on the abscissae as indicated by the breath number following the onset of rebreathing. The number of twitches increases rapidly when first recruited but more slowly as the stimulus continues to increase. Only when the curve of the total number of twitches of all fibers is plotted against increasing stimulus is there any correspondence with the curve of tidal air which is of course dependent upon the strength of the inspiratory contractions. [ATkinson, BRown and Gesels: Amer. J. Physiol. 129, 303 (1940).] tension of a single twitch, yields but a small amount of gradation as compared with the possibilities of recruitment. Admitting that the diaphragm has thousands of muscle units and that a finely graded contraction can spread progressively from a single unit at the close of apnea to all remaining units during the height of hyperpnea, it is clear that the gradation producable by recruitment may be many thousand 
times finer and greater than that of frequency alone. The force of that statement is readily appreciated by listening to the diaphragmatic potentials as muscular activity passes through this range- from complete silence to maximum hyperpnea. The mechanisms by which the slowly augmenting contraction may theoretically be accomplished will be discussed in other sections of this paper.

Attention has already been called to the similarity of appearance of the slowly and rapidly augmenting activity patterns. The rapidly augmenting

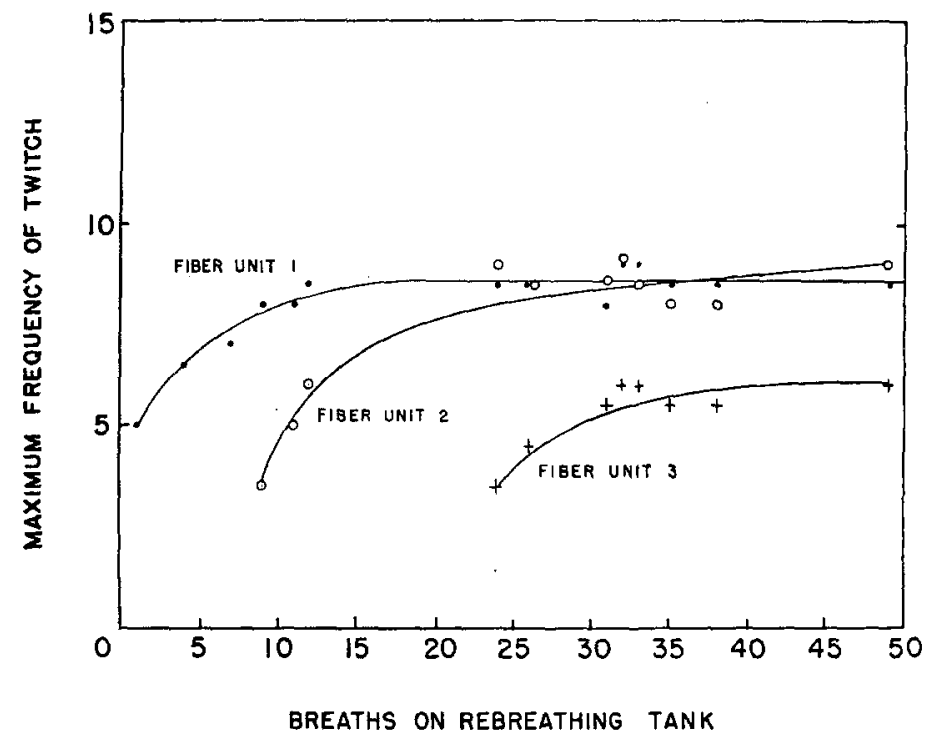

Fig. 12. Maximum frequency of twitch at the height of inspiration, plotted on the ordinates, against increasing chemical stimulation indicated by the number of breaths on the rebreathing tank on the abscissae. The lack of correspondence between the twitch of frequency of any single unit and the curve of tidal air gives significance to the parallelism of sum total of twitches and tidal air in Fig. 11. [ATKINsos, Brows and Geserc: Amer. J .Physiol. 129, 303 (1940).]

expiratory contraction is but a mirror image of the slowly augmenting inspiratory contraction. The contraction begins with a burst of force and the weakening is but a reversal of the augmentation of contraction. The forces producing this sudden burst of force and its subsequent weakening will be considered in greater detail under another heading.

Changes in strength of the steady state contraction depend upon the number of units twitching and the frequency of twitch of the respective units which may vary extensively. These contractions will also be considered in greater detail below.

In summarizing the activity pattern of inspiratory contractions we may say that frequency of twitch and recruitment and decruitment of active muscle units are the basic mechanisms upon which the integration of breathing are built. These tools are not only employed in each eupneic respiration to meet changing mechanical resistance but they are used to compensate for chemical 
threats such as lack of oxygen or excess of carbon dioxide. As we see the problem of respiratory control, it consists of determining the specific forces and machinery which bring about an orderly adjustment of frequency of twitch and of the number of active muscle units twitching. The following section showing the existence of an orderly system of frequency and recruitment adjustments running throughout the respiratory reflex arcs, naturally leads one to attempt a simple and fundamental interpretation of the respiratory act.

\section{Central Activity Patterns.}

The only conceivable method of studying the nervous integrating mechanisms of breathing is to register their activity patterns by electrical methods. This was done by a systematic exploration of the brain stem including regions well below and above the obex of the medulla with extremely fine insulated needles, bared only at the very tips [Geseld, Bricker and Magee (12)]. While listening for respiratory potentials with the aid of a loud speaker in electrical circuit, the needle electrodes were intermittently advanced about $0,1 \mathrm{~mm}$. at a time into the substance of the brain. When respiratory potentials in phase with either inspiration or expiration were heard a photographic record of the discharge and tidal air changes was made (see fig. 13 and 14). A small lesion about $1 \mathrm{~mm}$. in diameter was then placed by the passage of a weak galvanic current between the exposed needle points. This served to identify the source of any given set of action potentials on later histological examination. The slightest movement of the electrodes during exploration would frequently give rise to a change from inspiratory to expiratory potentials or vice versa, indicating a high selectivity of the electrodes.

A preliminary speculation on the type and original source of potentials likely to be encountered in the brain will assist the analysis of potentials to be described. Like those of muscle, the central potentials are divided into inspiratory and expiratory discharges according to the phase of breathing during which they occur. Obviously, inspiratory motor cells must discharge to activate the inspiratory muscles and logically, this discharge may be expected to be identical with the discharge of corresponding inspiratory muscles. Records of the activity of the diaphragm, of the phrenic nerve, and of the ventral horn cell, accordingly show the same triangular configuration of frequency activity and the typical triangular fusillade indicative of a rising coincidence of nerve cell activity from recruitment (see fig. $13 \mathrm{~A}, \mathrm{~B}, \mathrm{C}$ and $\mathrm{D}$ ). The ventral horn cells are in turn activated by impulses arriving by the reticular spinal tracts from the reticular cells near the obex of the medulla. These structures in turn show the same slowly augmenting patterns of frequency and recruitment as noted in the lower motor units (see fig. $13 \mathrm{E}$ and F). Contraction of the inspiratory muscles must give rise to a stretching of GolgI endings due to a rising tension in the tendinous insertions. Potentials may, therefore, be expected 

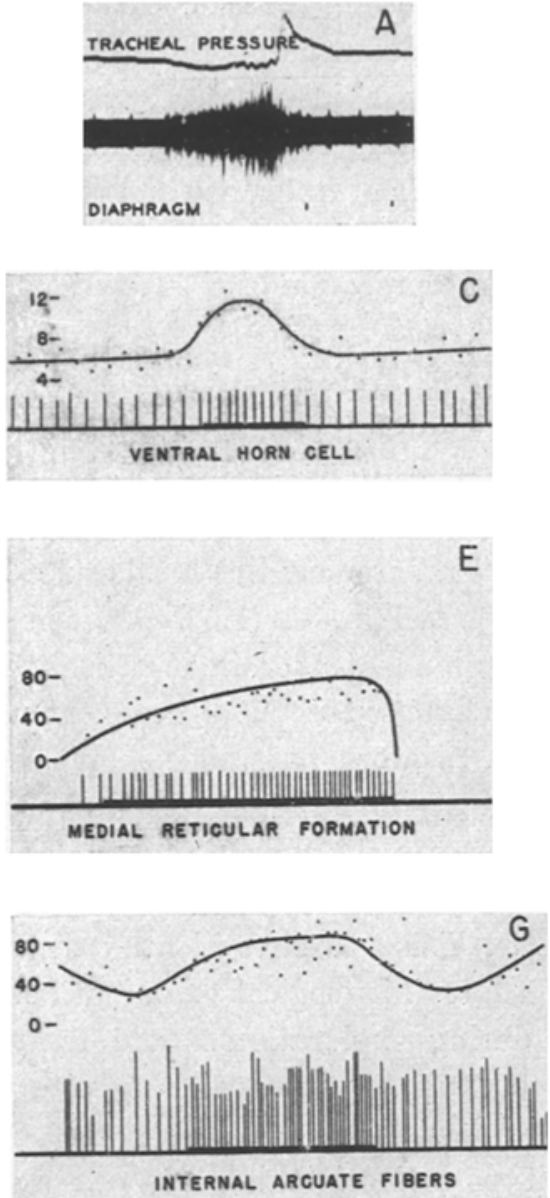
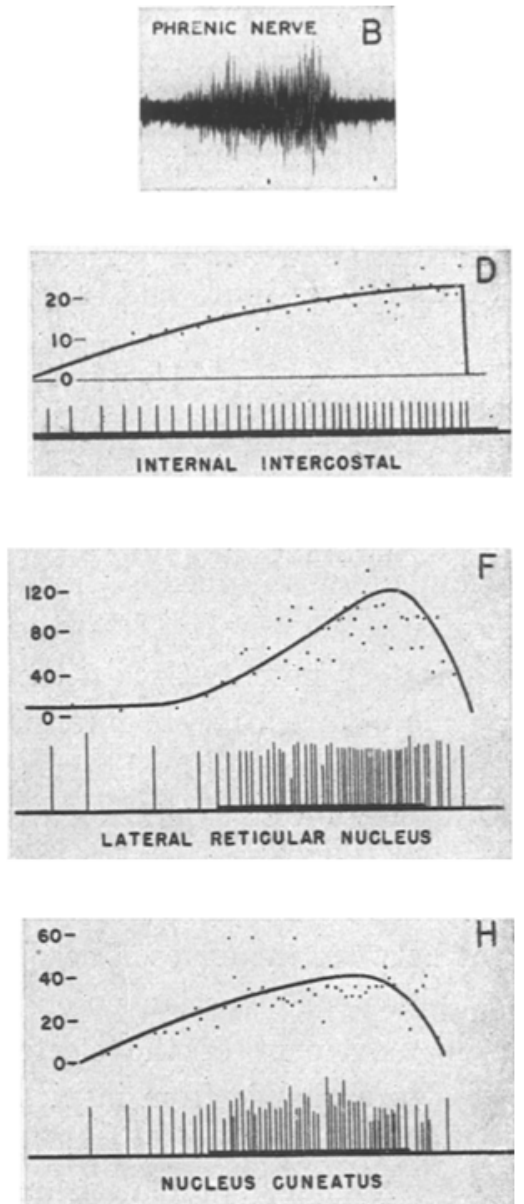
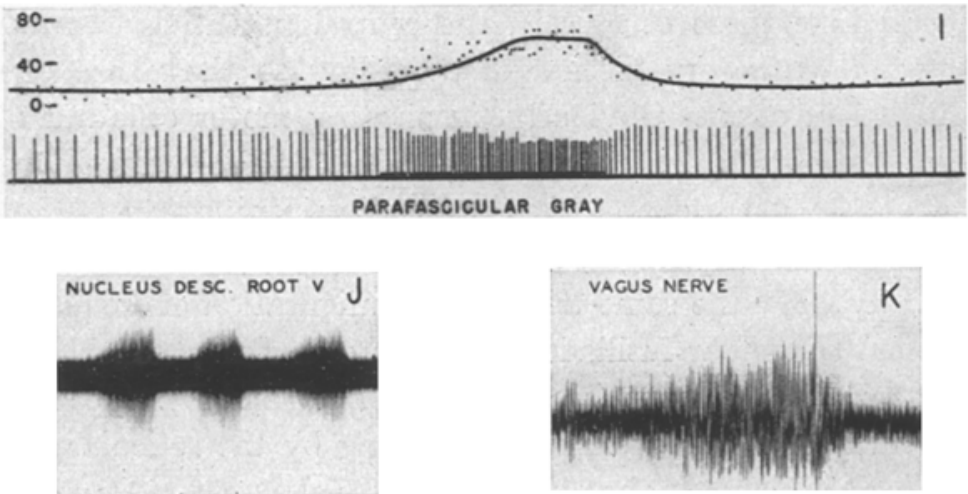

Fig. 13. Inspiratory activity patterns, peripheral and central, showing the typically slow augmentation of frequency and recruitment activity. The universal incidence of these basic phenomena indicates a similarity and simplicity of motor integration at all stations in the inspiratory are. [GEgELL, MAGEE and Bricker: Amer. J. Physiol. 128, 615 (1940).] 

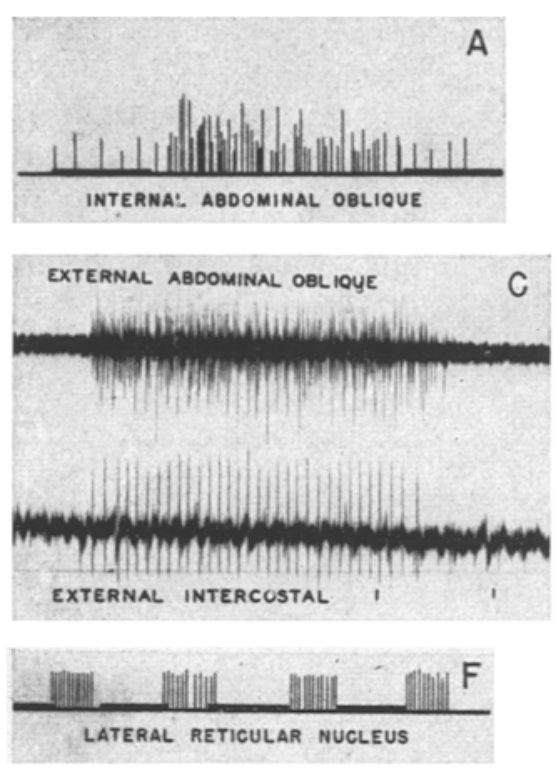
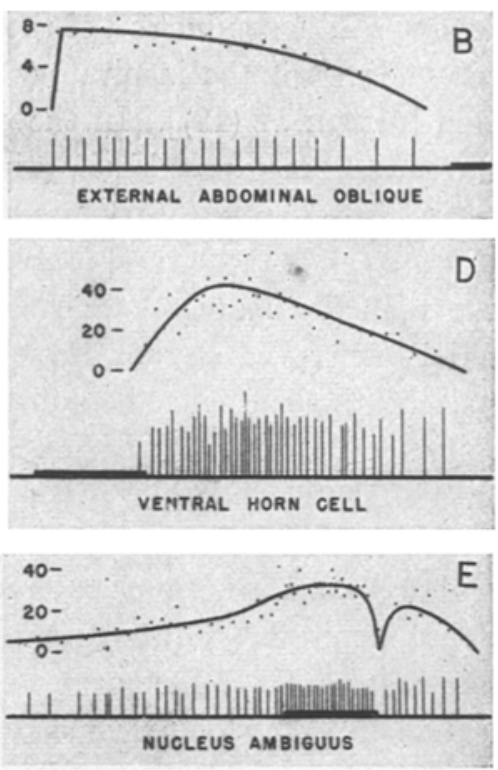

G
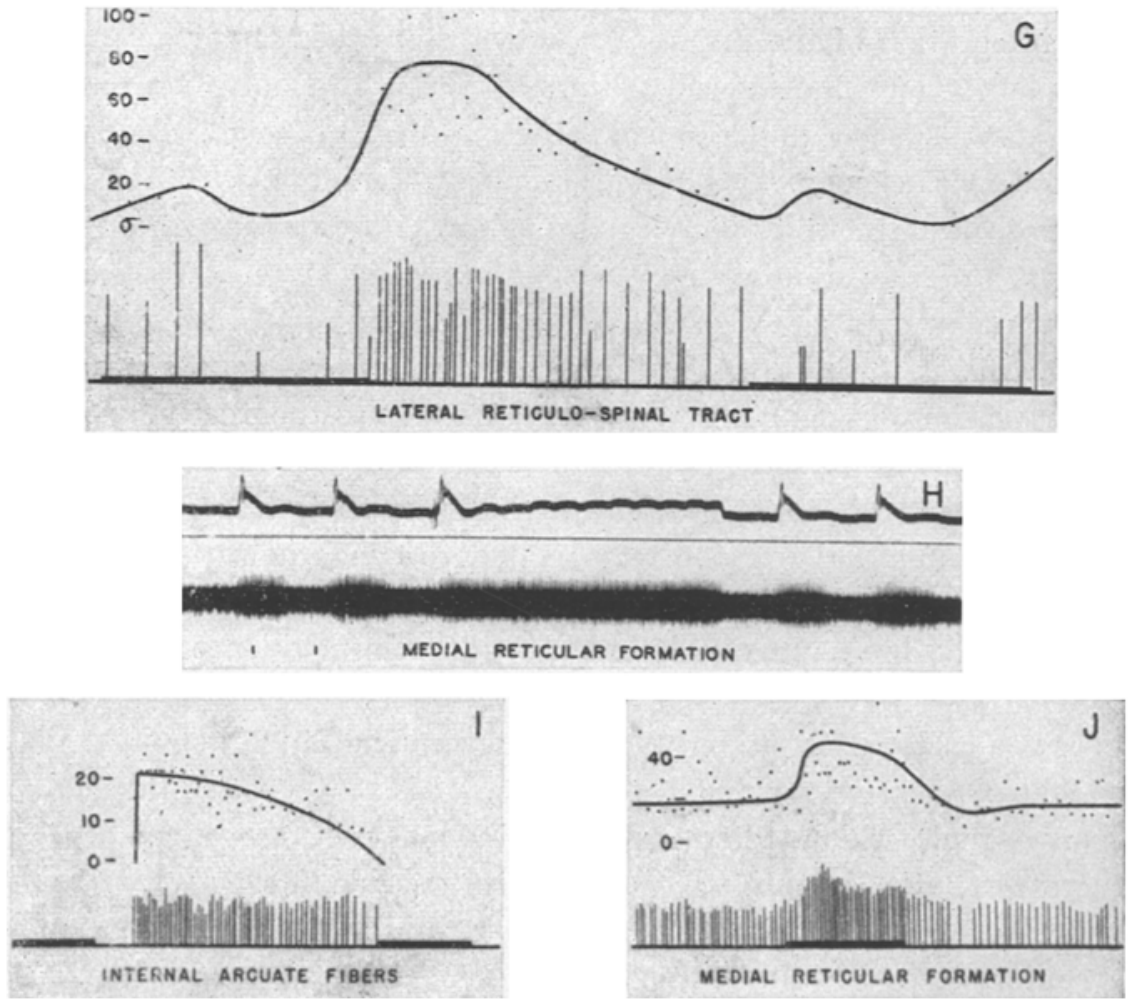

Fig. 14. Expiratory activity patterns of motor, sensory and intermediate stations of both rapidly augmen. ting and steady state types. Fig. F and G are of special interest in showing discrete discharges from both inspiratory and expiratory structures at a single setting of the electrodes. Because the electrodes are bighly selective such records indicate the proxinity of inspiratory and expiratory structures, one with the other. The prolongation of the steady state activity in fig. $H$ is also worthy of note. [Gesel, Magee and Brtcker: Amer, J. Physiol, 128, 615 (1940).] 
along the path of conduction of these signals (see fig. $13 \mathrm{G}$ and $\mathrm{H}$ ). The muscle spindle endings of the inspiratory muscles are presumably protected by contraction [Mathews (13)]. In the expiratory muscles both Gougr and muscle spindle endings may possibly be stimulated by the inspiratory contractions and, also give rise to inspiratory potentials. The inflation of the lungs must of necessity stretch the vagal proprioceptive endings which discharge in proportion with the degree of their stretch. It is, therefore, reasonable to expect potentials wherever any of these signals travel and because the distortion of each of the specialized endings must undoubtedly correspond with the intensity of the inspiratory contractions, inspiratory activity patterns similar to those of the inspiratory muscles are to be anticipated (see fig. $13 \mathrm{I}$ and $\mathrm{K}$ ). Inspection of sample records from such structures as the reticular formation, internal arcuate fibers, nucleus cuneatus, parafascicular gray and vagus nerve show that inspiratory muscular activity is mirrored in respiratory units throughout the respiratory reflex arcs.

This similarity of muscular activity and of activity of the central integrating structures is a most encouraging observation, for it seems to promise that the integration of the respiratory act or any other motor act, may be a very simple phenomenon indeed. After all, the use of only two exceedingly elementary tools such as frequency and recruitment could readily resolve itself into a most elementary integration. It is, therefore, with equal interest that we note a harmony of activity throughout the expiratory reflex ares in the existence of the rapidly augmenting and steady state activity along the expiratory reflex arcs (see fig. 14).

Just as it is the sum total of muscle activities that counts in work performed or tension developed, so it is the sum total of neurocellular activities that is important in nervous integration which brings this adjustment about; and so far as we are able to see parallelism of activity along either the inspiratory or expiratory reflex arc is in no way related to synchronization or temporal arrangements as have heretofore been assumed to exist. In all our electrical soundings of the brain we could find no supporting evidence for synchronization or any other temporal scheme of integration. There are no indications whatever of a correspondence between the frequency of discharge of one neuron and of that of the next neuron in the chain. It is rather the total number of impinging signals which determines the frequency of nerve cell discharge. In other words the quantitative control responsible for activity gradation and coordination is a number and not a temporal factor. This concept is in direct conformity with our theory of synaptic drive as opposed to that of synaptic transmission based on temporal arrangements. In our opinion motor integration is an example par excellence of a smoothly operating process resulting from a train of asynchronous activities, a point which we shall attempt to develop as we proceed. 


\section{The Respiratory Center.}

In formulating our concept of the respiratory center the statement of Sherrington and his colleagues that the "Principle of Convergence is the basis of coordinated function of the nervous system" must serve as our main guide, for certainly the respiratory act is a highly coordinated nervous integration which is dependent upon the convergence of a host of varying types of signals. This heterogenous mass of signals coming from the carotid and aortic bodies, the GoLGI and muscle spindle endings, the vagal stretch receptors, the recruiting and reciprocating collaterals and the fiber tracts descending from above, all converges upon the scattered reticular cells in the medulla. These cells are then assumed to convert these signals into a new and orderly group which travels in turn down the reticulo spinal tracts to activate the respiratory muscles in the orderly way already described.

On the basis of neurohistological findings just described and experimental and pathological findings the reticular cells have long been suspected as being the coordinating stations for respiratory control. Thus in 1847, LoNGET (14) destroyed the pyramids and the restiform bodies in the neighborhood of the vagus nucleus, without interfering with breathing, but "the isolated destruction of the 'faisceau intermédiaire du bulbe', at the same level, caused instantaneous suspension of breathing". He added that this bundle contained numerous nerve cells and gray matter and for that reason appeared to be well suited to serve as a center of some particular function. According to Mislawsrey (16) this appears to be the medial reticular formation. In 1858 Flourens (16) recognized the dorsal part of the reticular formation just above the obex as the center. In 1885 Mislawsky (16) placed it in the reticular formation mainly internal to the roots of the hypoglossal nerves which would correspond to the medial reticular formation. Destruction of this area by pricking abolished the respiratory movements immediately while stimulation of the area electrically resulted in a more rapid rate of respiration. In 1890 LABonde (17) found that destruction of a small region just above the point of the calamus stopped respiration. This perhaps corresponds to the medial reticular formation. GaD and Marinesicu (18) (1892) noted that only destruction of the reticular formation stopped respiration. Cauterizing Flourens vital node, the external part of the alae cinereae and the solitary bundle was without effect (regions of Fiourens, Longet, Schiff, Gierke and Mistawsky). Electrical stimulation of the reticular formation produced an acceleration of breathing. In 1894 Arnherm (19) found that stimulation between the nucleus of VI and the calamus accelerated respiration if the electrodes touched the reticular gray. Koнnstamm (20) in 1900 considered the reticular formation as an important regulatory mechanism for the nuclei supplying respiratory muscles. After section of the spinal cord in its upper part he obtained a degeneration of the 
reticular formation in the region BECHTEREW (21) described as his center (1908). On the basis of pathological findings in man FinLey (22) (1931) concludes that one of the main integrative levels for respiratory impulses lies in
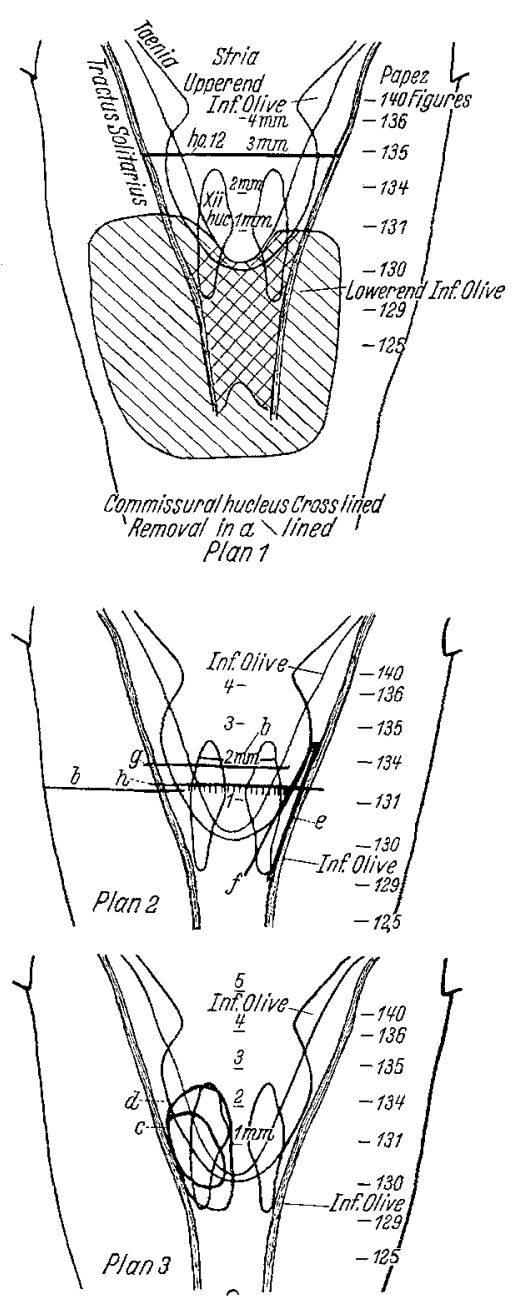

Fig. 15. Plan to scale of the medullary area prepared as described in the text, showing the relative positions of the important structures referred to in the text, and also the levels of the sections published by Papez. [HENDERson and CraigIE: Amer. J. Physiol. 115, 520 (1936).] the reticular formation. According to PaPez (23). and Aluen (24), the reticulospinal tracts are employed to conduct the impulses to the motor cells of the respiratory muscles.

More recently Henderson and Crargue (25) have removed the commissural nucleus completely without disturbance of breathing thus disposing of CaJaLs (26) contention that the respiratory center resides in this structure and at the same time supporting the suggestion of BECHTEREW that the center lies in the reticular substance ventral to the nucleus of XII. By other transverse and sagittal lesions they limit the respiratory cells to the medial third of the medulla from about the level of the junction of the middle and upper thirds of the hypeglossal nucleus to the pyramidal crossing below.

These experimental findings indicating that the reticular formation serves as the main station for the integration of the respiratory signals are amply confirmed by the electrical soundings of Geseli, Bricker and MAGeE (12). No other structures yielded respiratory potentials in such abundance. With the aid of these potentials they set the upper limit of the center across the upper quarter of the $\mathrm{N}$. hypoglossus, $\mathrm{N}$. ambiguns and N. fasciculus solitarius (see fig. 16). The incidence of respiratory potentials extending continously from the medulla through the cord prevents similar establishment of the lower border, but a line marking the disappearance of the reticular formation potentials places the lower border at the cephalic edge of the pyramidal decussation. The frequent occurrence of potentials in the lateral reticular nuclei marking a more lateral extension of the respiratory center is the only point of difference between our results and those of HENDERson and Craigre.

The diffuseness or compactness of a center is a matter of some interest in relation to localization upon which the experiments of Geseld, Brrcker and MAGEE seem to shed some light. 
"Dense riddling of the entire lower medulla in some animals seemed to have no effects whatever on breathing and permitted extensive observations. To be sure, the animals deteriorated, some very slowly and others rapidly, but previous decortication seemed more related to collapse than did sounding for potentials. The fineness of our electrodes could easily account for the lack of damage but repeated galvanic lesions seemed to produce only momentary effects while the current was flowing. This immunity to damage is probably related to the structural and
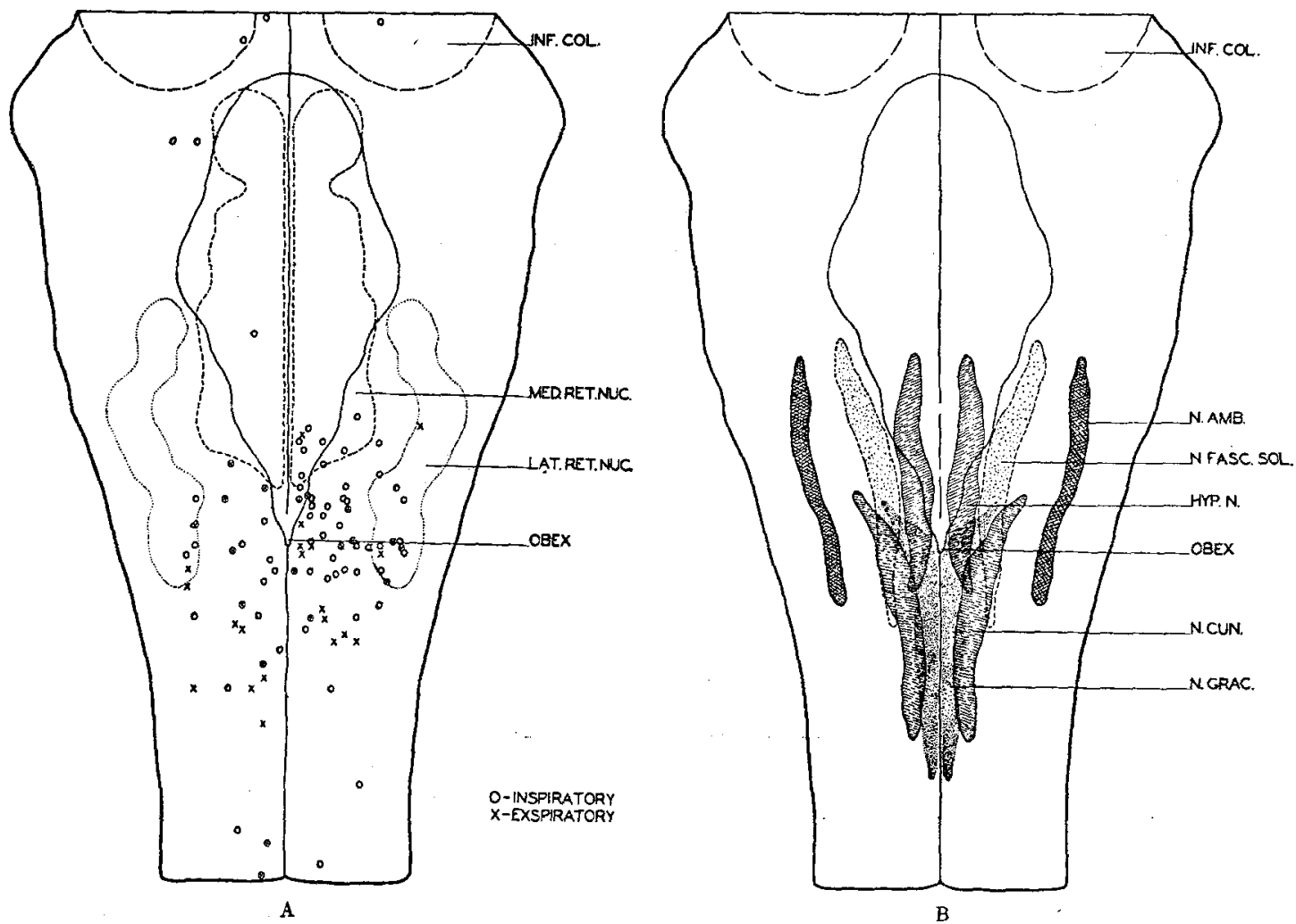

Fig. 16. Gross localization of the respiratory center in the $\operatorname{dog}$ by the method of electrical sounding. The location of inspiratory potentials are designated by an open circle and of the expiratory potentials by an $X$. Where both sets of potentials were obtained at one setting of the electrodes the position is marked by an $\mathrm{X}$ enclosed within a circle. This combination is likely to look like a solid circle. [GESELL, BRIOKER and Magee: Amer. J. Physiol, 117, 423 (1936).]

functional organization of the reticular formation. The impression we have gained is that the potentials are relatively few and scattered and that the anatomical description 'scattered reticular gray matter' is descriptive of its function as well. The relaive scarcity of reticular potentials, the large size and spacing of the cells, considered with the copious innervation of muscles as represented in the ventral column of the cord suggest a multiple innervation of the ventral horn cells with a high step-up ratio. '- . . ' - The absence of a systematio or localized arrangement of inspiratory and expiratory potentials, as plotted in fig. 17, indicates a lack of grouping of cells into inspiratory and expiratory nuclei and changing potentials with progressive penetration of the electrodes yields the same conclusion. This point is illustrated by an example of a single sounding of $3 \mathrm{~mm}$. extent, which successively tapped off seven sets of potentials in the order given-inspiratory, expiratory, expiratory (new set), regular frequency without respiratory rhythm, expiratory, inspiratory, and expiratory. The previous conceptions of compact, definitely localized inspiratory and expiratory centers, held by some investigators, probably should be revised in the light of our findings." 
In more recent studies on the respiratory center of the cat, Prxts, Magoun and RANSon (27) also localized the respiratory center in the formatio reticularis by exploratory stimulation as illustrated in fig. 16. In disagreement with the

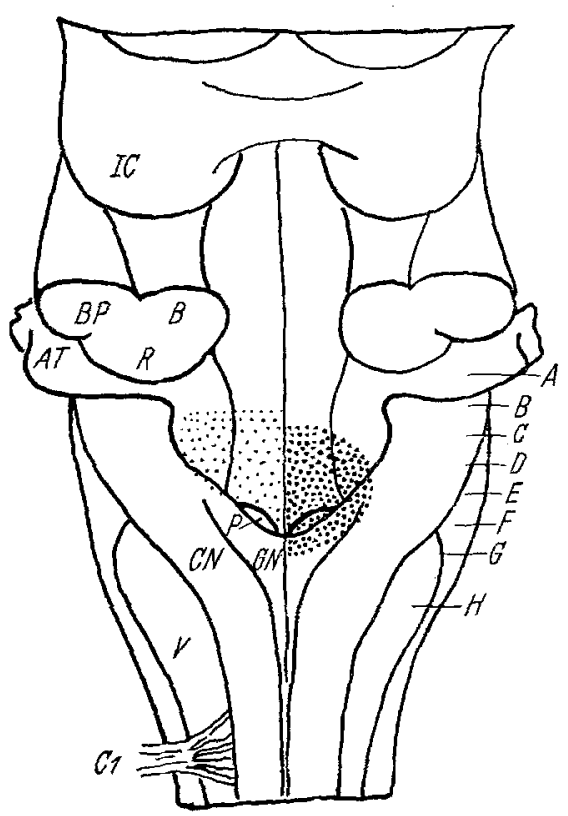

Fig. 17, Dorsal view of the lower brain stem of the cat with the cerebellum removed. The location of the maximal inspiratory (heary stippling) and maximal expiratory (light stippling) responses is diagrammatically shown projected on the floor of the fourth ventricle. To avoid overlapping, the expiratory reactive region is indicated only on the left, inspiratory onfy on the right. On the right side of the figure the lettering from $A$ to $H$ indicates the level of the sections shown in fig. 91 and 92. Acoustic tubercle, $A T$; brachium conjunctivum, $B$; brachium pontis, $B P$; first cervical dorsal root, $C_{1}$; ouneate nucleus, $C N$; gracile nueleus, $G N$; inferior colliculus, $I C$; area postrema, $P$; restiform body, $R$; tuberculum cinereum, $V$. [PITTs, Magoun and Ranson: Amer. J, Physiol. $126,673(1939)$.] electrical sounding experiments they claim a delineation of this center into sharply defined expiratory and inspiratory centers. Another probable interpretation of their results will be discussed in detail in section 24 .

\section{The Origin of Respiratory Activity Patterns.}

In section 4 on the activity patterns of the motor, sensory and intermediate units we attempted to explain why the activity is the same along the full extent of either the inspiratory or expiratory arc. We now inquire more specifically how each of the three activity patterns originates. Theoretically they may arise in two ways- centrogenically and reflexogenically. They might develop centrogenically from some peculiar structural arrangement or some physico chemical property of the reticular cells which forces these cells to discharge in their characteristic manner. If, for example, the reticular cells are the pace setters of inspiratory activity, it is clear that the entire inspiratory arc would be forced to follow the activity patterns wh th these cells initiate. The inspiratory anterior horn cells and the corresponding muscles would necessarily respond in kind to the motor impulses dispatched from above by the reticular cells and the setting up of stresses and strains within the inspiratory muscles would stimulate their proprioceptive endings in similar fashion and thus complete the train of like inspiratory activities at all of the sensory stations of the inspiratory ares.

On the other hand the slowly augmenting pattern could be a purely reflexogenic phenomenon. We may picture the reticular cells as beginning inspiratory activity with a steady state discharge of relatively low intensity. The inspiratory muscles contract at once. The so distorted Goxal and vagal endings dispatch an increased volume of signals back to the reticular cells and stimulate 
them to greater activity. The newly augmented contraction then increases the proprioceptive distortion and superimposes a second augmentation of activity on the reticular cells and thus the contraction continues to increase up to the end of the inspiratory phase. :

Direct experiments [Gesell, Atkinson and Brown (28)] show that slowly augmenting inspiratory activity originates both centrogenically and reflexogenically, and that both mechanisms have a mutual interaction leading to an accentuation of the inherent inspiratory discharge. The proof of the existence of a centrally inherent inspiratory activity is provided with the

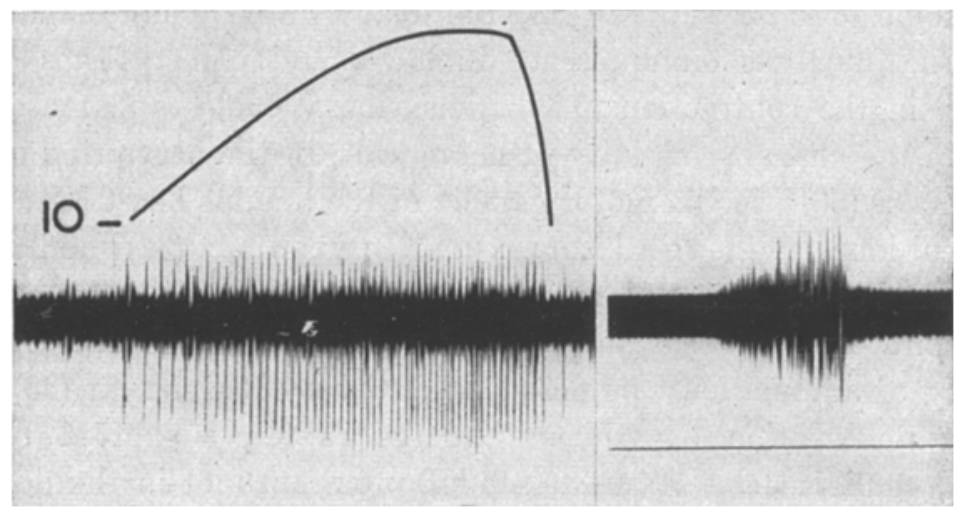

Fig. 18. Action potentials of the phrenic nerve of the dog during complete curare paralysis designed to remove all periodic respiratory afferent impulses. The persistence of the slowly augmenting frequency of discharge of the individual nerve fiber and the continuance of the slowly augmenting recruitment of additional active fibers demonstrate the existence of a central mechanism capable of transforming steady drives into the characteristic inspiratory activity found in the physiologically intact animal. [GESELL, ATkInson and Brown: Amer. J. Physiol. 128, 629 (1940).]

help of paralytic injections of curare which abolishes all periodic proprioceptive reflexes. The animals are made apneic by artificial ventilation. The ventilation is then stopped and the chemical stimulus allowed to rebuild and reinitiate activity of the respiratory center which is then followed electrically in the motor nerves of the respiratory muscles. Fig. 18 is an example of phrenic nerve activity in a totally paralyzed dog. Since both acceleration of the discharge frequency and recruitment of nerve cell activity are invariably demonstrable in these curarized animals, it must be concluded that there is some central mechanism capable of discharging rhythmically in the characteristic slowly augmenting manner under a steady state environment and steady state reflexogenic drive. This mechanism of centrogenic augmentation though tentatively placed in the reticular cells of the medulla might also reside in the anterior horn cells. Proof of the reflex bolstering of the inherent central discharges will be presented in the sections dealing with the afferent drives.

Of the two expiratory patterns of respiratory activity only the steady state type was demonstrable in the absence of periodic sensory impulses. 
It was, therefore, tentatively concluded that this type of activity is dependent upon peculiar properties or arrangements of cells in the expiratory half-center differing from those of the inspiratory half-center, and that the rapidly augmenting type is dependent upon the play of periodic proprioceptive signals on the steady state activity. Proof of the reflexogenic origin of the rapidly augmenting expiratory activity will be presented below.

\section{The Electrotonic Theory of Nerve Cell Activity.}

The unbelievably high incidence of periodicity in chemical and physical systems (some go so far as to say that the steady state is non-existent [HEDGEs and Meyers (29)], its omnipresence in lower and higher forms of life, its occurrence in the contraction of cilia, flagella, vacuoles and muscle and its presence in the changing activity of nerve cells, in the nerve ring of the jelly fish [Romanes (30)], in the motor ganglia of the limulus heart [Carlson (31)] in the vagal lobes of the fish [Adrian and BuytendiJK (32)] and most of all in the medulla of the mammal, requires the consideration of automatic activity of the respiratory center in any complete system of respiratory integration. The theory of automaticity proposed originally by Rosenteal (33) as a conclusion of denervation experiments in the rabbit, supported by MARcKwatd (34), confirmed by Heymans and Bouckaert (35) in the dog, and rid of the objection arising from continued irritation of the cut afferent fibers by Adrian and BurtendiJk (32) now stands on firmer ground, and allows more detailed speculation on its nature.

In looking for a mechanism of automatic activity of the respiratory center we have re-employed an electrotonic theory of nerve cell discharge proposed as early as 1926 [GESELL $(36,37)]$. In subsequent years this theory has found increasing experimental support and promises to play a most important role in neurophysiology. In its present form, it is based on two well established facts- first, that metabolic gradients give rise to electrotonic currents, and second, that such currents if sufficiently strong produce a rhythmical discharge at their point of emergence. According to our theory, [Gesert (38, $39,41)$ ] current flows within the nerve cell from regions of high metabolism in the dendrites through the cell body to a region of low metabolic rate at the axon hillock (see fig. 19). Meeting high resistance at this point it leaves the cell at the axon hillock, and returns in the immediate environment back to the numerous dendrites. At the site of emergence the neuromembrane alternately breaks down and rebuilds, producing periodic activation and recovery, thus mimicking the rhythmical discharge so readily demonstrated in the iron nerve model of LiLuie (42).

It is our belief that this simple mechanism of self excitation by self engendered electrotonic currents in the respiratory nerve cells may have a 
universal application to automatic biological activity in general, for we envisage the electrotonic current as the master tool of nervous integration. Not only is it thought to generate rhythmical signals in the respiratory center but to be the possible source of rhythmical firing wherever it occurs-sensory receptors, nerve cells in general, heart muscle etc. It is known to be the means of transport of signals in nerve fibers and muscle and would well be considered in the explanation of brain waves, so intensively studied at this time.

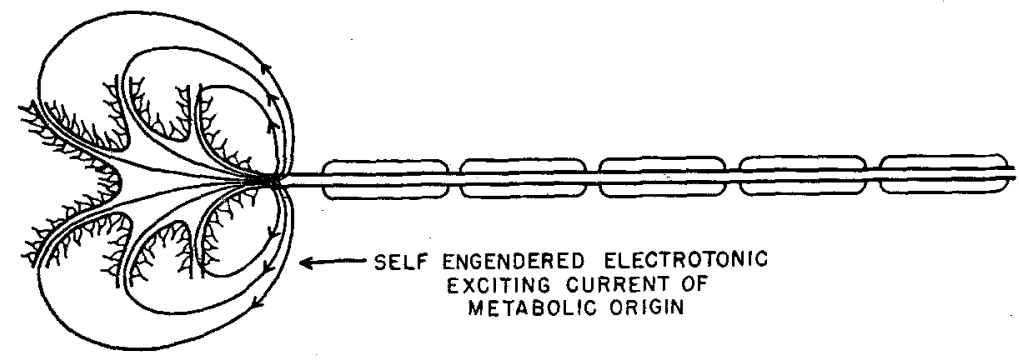

A STEADY ELECTROCHEMICAL DRIVE PRODUGING A. STEADY STATE DISCHARGE

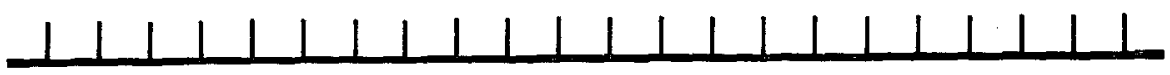

Fig. 19. An electrotonic mechanism of nerve cell discharge based on the existence of metabolic and potential gradients. Conditions peculiar to the cell generate a steady flow of current and confine it to the course shown by the arrows. The point of outflow is at the axon hillock which the current discharges at the arbitrary frequency shown in the lower record. [GESELL: Science (N.Y.) 91, 229 (1940).]

Bold as such a view may seem it has the tremendous advantage of narrowing the origin of rhythmicity to a single tool, and unifying and simplifying many of our concepts. Such broad hypothesis rests on substantial biological support for it has long been known that the quiescent ventricle of the turtle's heart responds with rhythmical activity to a constant flow of current. GARTEN (43) has shown a similar rhythmical response in the isolated nerve, so often seen in the tetanus produced in the demonstration of PrLÜGers table. The results are not surprising for they occur in LrLLtes (44) unbelievably life-like inorganic nerve model under the influence of a uniform galvanic current. But the clearest proof of the electrotonic theory of nerve cell discharge comes from Ostenhout and Hruc (45) who applied chemical irritants to the Nitella and observed the rhythmical electrical discharges which resulted (see fig. 20 and its description). They concluded "that a negative variation may be started whenever it is possible to set up along the protoplasm a gradient of potential difference sufficiently steep to produce the necessary outflow of current. Successive variations are thus set up." They stated further that "These facts suggest the possibility of producing successive variations in muscle and in nerve in somewhat the same way as in the Nitella. Experiments are 
being made to test this suggestion". Shortly thereafter Adrran (46) confirmed this suggestion and found that cut nerve fibers respond rhythmically to their own current of injury. More recently we have learned of unpublished results

(A)

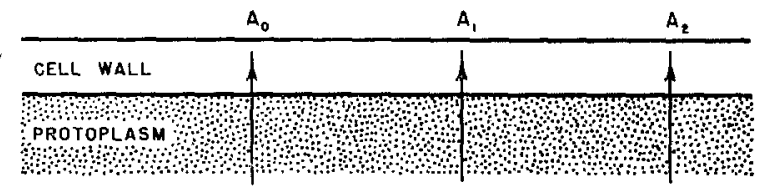

(B)

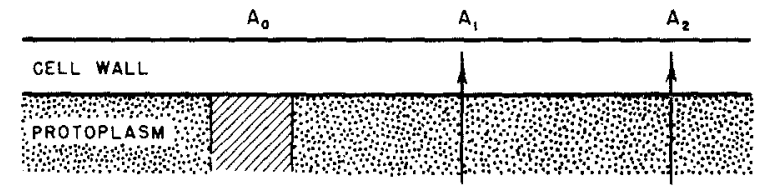

(C)

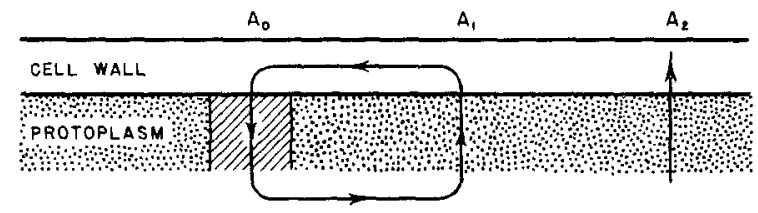

(a)
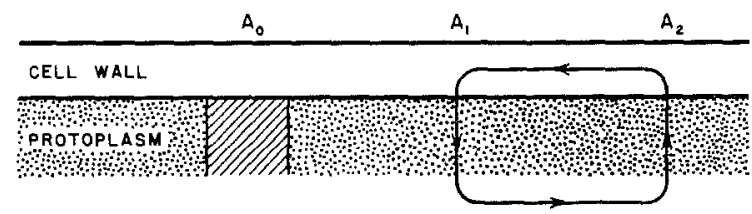

(E)

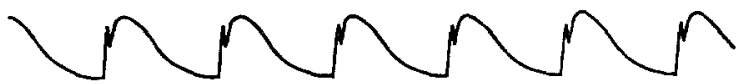

Fig. 20. The mechanism of the rhythmic discharge of the Nitella produced by localized chemical irritation. This irritation, fig. B, generates a circulating current which excites the intact cellmembrane at its point of outflow ( $A_{1}$ fig. $C$ ). The point of outflow then becomes electrically negative and initiates a new outflow at $A_{2}$ fig. $D$ and thus the disturbance is propagated. The electrical record below shows the rhythmical activation of the Nitella of the constant electrotonic current of injury set up at $A_{0}$. [OsTERHOUT and Hixx: J, gen. Physiol. 13, 459 (1930).]

of Kenneth CoLE on heart muscle cultures which he has kindly allowed us to cite. At the stage when muscle cells develop rhythmicity a careful exploration of the immediate environment of the muscle with monopolar exploration reveals local regions of negativity. These negative spots could well provide the local circuit already alluded to in the experiments of Osterhout and HiLL on the Nitella and initiate the contractions which Cole has observed. 


\section{The Electrical Requirements of Electrotonic Excitation.}

A basic theory of this nature demands a consideration of the electrical conditions which exist in and about a nerve cell to determine whether or not they meet the necessary requirements. First of all some logical source of potential difference and confinement of current to the channels already described must be presented. This is necessary because the present views of nerve cell activity assume that impulses are propagated along the dendrites and cell bodies just as they are in the nerve fiber (see current monographs and journals). We have, therefore looked for conditions which would tend to maintain an electrotonic current in well directed external and internal circuits and also for conditions which would oppose the development of local circulatory currents along the dendrites and cell body such as are demanded by impulse conduction in the nerve.

The bigh metabolic rate of gray matter as compared with white [HoLmes (47) places it as 10 to 1] serves as the basis of our conception. According to some, this metabolic gradient depends upon the distribution of synapses, for metabolism is highest and circulation is most adequate where dendrites and their synapses are most numerous [Cratgie (48), Holmes (47), Dunning and WoLFF (48)]. Since the synapses virtually form a continuous covering over the cell body and dendrites, it must of necessity follow from the law of surface- mass relation that the synapses are most highly concentrated where the calibre of the dendrites is the smallest. The metabolism of the neuron should on this basis be highest in its fine dendritic extensions. GErard (49, $50,51)$ on the other hand is inclined to discount the influence of the synapses upon nerve cell metabolism and rely rather on the simple surface mass relation of the dendrites themselves to explain the higher metabolism of grey matter. Whatever the outcome of this difference of opinion may be, each tapering dendrite, widening as it approaches the cell, should provide a potential drop and a lowering of the resistance to ease the flow of current towards the cell body. Like tributaries to a stream, they bring their current, which now flows still more easily and more diffusedly in the widened lake of cytoplasm. Confined by the barriers of the neuromembrane plus the double membranes of the tightly packed synapses impinging on the neuron, the current converges in concentrating lines toward the axon hillock which lies at the end of the potential gradient. The excessive longitudinal resistance of the exiting neuraxon, the absence of further potential drop in the neuroaxon, and the absence of synapses and their electrical resistance at the axon hillock shunts the current through the neuron membrane at this point into the outer environment of the cell. Thus a current generated by the activity of a great expanse of cellular surface is concentrated before its exit at the axon hillock. Ideal conditions for local excitation are thus obtained. 
Here may enter a most significant role of the neurodendrites. Highly negative, and spreading like tentacles in all directions, they absorb the returning current. More significantly, they contrive to sweep the current in large circuits and add their bit to the avoidance of highly localized membrane circuits so fatal to our hypothetical functioning of nerve cells. By thus offering a tremendous surface, a high dilution of returning currents is accomplished and the disorganizing effects catelectrotonic excitation and anelectrotonic blocking of outlying fibers are minimized. Moreover the synaptic covering of the dendrites, we suggest, works like the myelin sheath in preventing highly localized cireulating currents necessary for impulse conduction.

Highly active and important as the dendrites seem to be in the establishment of potential drop, there must be adequate provision for metabolic exchange. The favorable relation of surface to mass in the fine and tapering processes and their extension into highly vascularized tissue have long attracted the attention of anatomists (Golgi) quoted by Kappers, Huber and Crosby (52). It is interesting, therefore, to look upon these delicate and little understood structures as generators and conductors of current flowing within the cell, as antennae for the absorption of returning currents, as combination villi and gills for metabolic exchange, as receiving stations for synaptic drive, and as controllers of potential drop. Ideal conditions are present for the nerve cell to function as an electrochemical generator of a self exciting current.

\section{The Gradation of the Self Engendered Electrotonic Excitation.}

Granted that the electrotonic current which we have described is capable of producing a continuous train of nerve cell discharges, it becomes necessary to determine how that frequency of discharge is graded and how it is interrupted to produce the rhythmical respiratory act. A gradation of the intensity of the electrotonic current would be a possible mechanism of frequency control. The direct proportionality of the frequency of discharge of the iron nerve model of LILLIE to the intensity of the voltage impressed supports that possibility and encourages us to look for comparable situations within the respiratory center. Due to the fact that the center is capable of direct chemical stimulation as well as reflex excitation, theory requires a search for two mechanisms of gradation of the electrotonic excitation current- an inherent electrochemical mechanism of metabolic origin and a reflexogenic mechanism possibly building upon the former.

Among the various situations which must be met by the respiratory system in its adjustments to changing requirements is an increased metabolism consequent upon a rise of temperature. In that connection the experiments of GARREY $(58,54)$ on the effects of temperature upon the frequency of the 
rhythmic discharges of the pace setting cardiac ganglion of the Limulus are significant (see fig. 21 A, B). They are particularly interesting in that Garrey determined the increased rate of carbon dioxide formation in the ganglion as well as the increased frequency of periodic ganglionic discharges and was

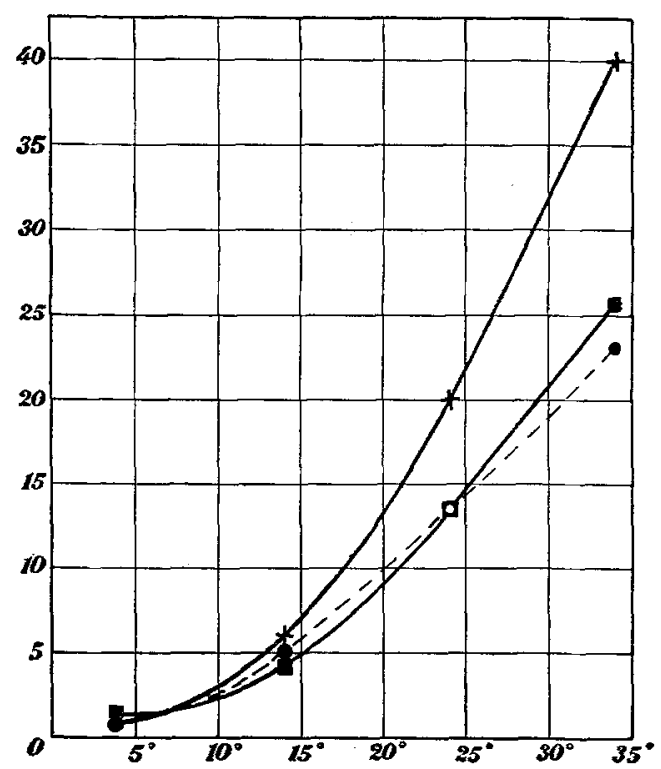

Fig. 21 A. Curves of the relative velocities of carbon dioxide production by the Limulus heart ganglion at different temperatures. [GARREY: J, gen. Physiol. 3, 49 (1920).] able to correlate the frequency of beat with the intensity of the gaseous meta-

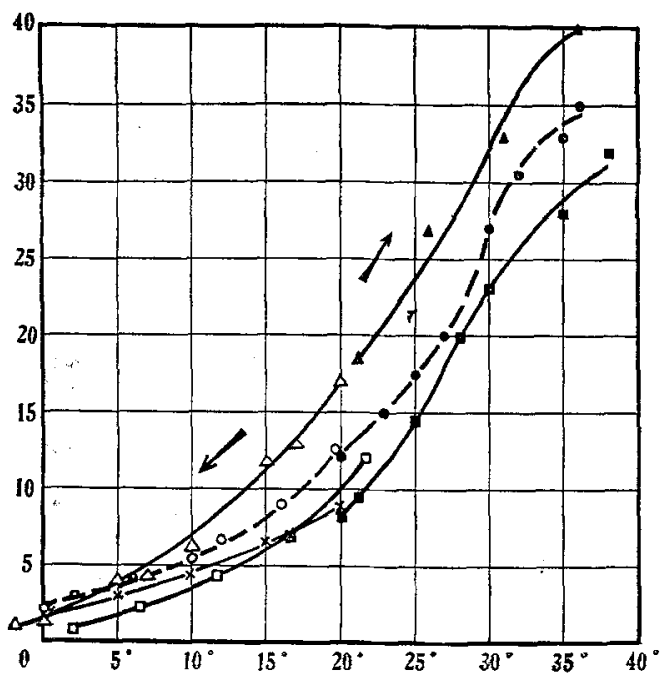

Fig. $21 \mathrm{~B}$, Curves of relative frequencey of discharge of the Limulus heart ganglion at different temperatures. [Garrey: J. gen. Physiol. 3, 41 (1920).]

bolism. The frequency of brain waves is known to show similar relations to temperature [HoAGLAND (55)].

Our interpretation of results of this kind are based on the experiments of Houmes in which he concludes that the rate of metabolism in the dendrites is ten times that of the neuraxon. Granting that a rise of temperature increases the normal rate of metabolism of the dendrites and neuraxon from 10 and 1 respectively to 20 and 2 a new intensity difference of metabolism, from 9 to 18, is established. Theoretically this means a doubling of the potential gradient and a doubling of the frequency of discharge of the axon billock. Thus we see the possibility of a simple regulative mechanism capable of controlling the activity of the respiratory center in proportion to the metabolic requirements as produced by temperature fluctuations.

The mechanism by which carbon dioxide stimulates the respiratory center must likewise be only a matter of conjecture. If it be assumed that carbon dioxide produces a greater degree of depolarisation of the neurocellular membrane at the finer dendrites where metabolism is the highest, than in the regions where metabolism is lower an extremely simple mechanism of control is at hand. The finer dendrites will turn more negative with respect to the 
axon hillock, the greater electrotonic current thus established will increase the frequency of discharge proportional to the existing pressures of carbon dioxyde.

\section{The Electrotonic Theory of Synaptic Drive.}

Granting that the central chemical control of breathing is basically a gradation of self engendered electrotonic currents we are virtually forced to look for an amplification of this gradation in the reflexogenic chemical control

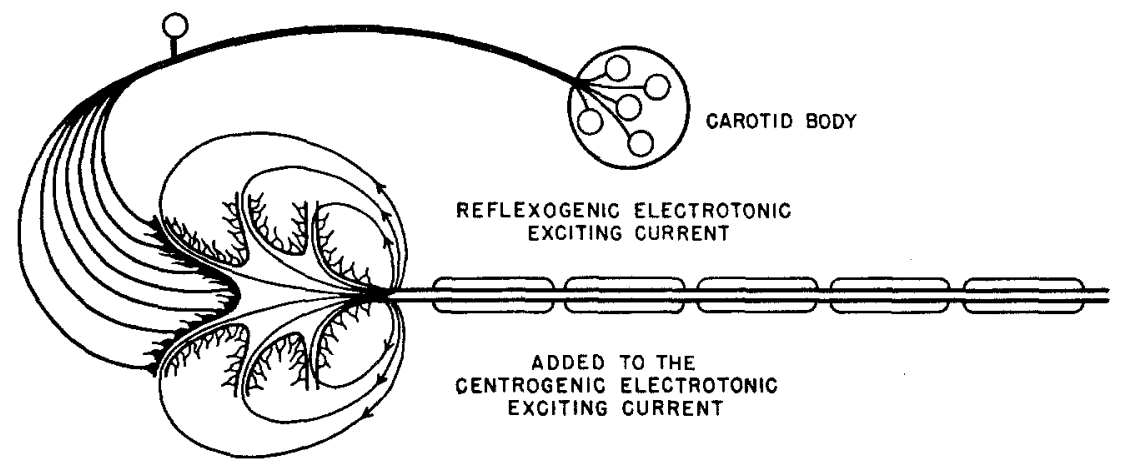

THE RESULTANT DRIVE PRODUCING A HIGHER FREQUENCY OF STEADY STATE DISGHARGE

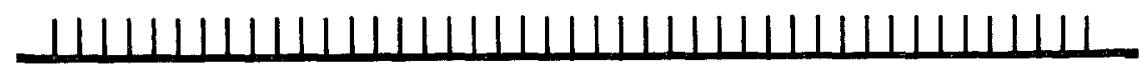

Fig. 22. An hypothetical schema of the synaptic activation of nerve cells. For simplicity only one type of signal is depicted as impinging upon the excitatory end of a reticular cell, namely those arising in the chemoceptors of the carotid body. These signals are assumed to produce a condition of local negativity in the immediate vicinity of their impingement. This negativity adds to that aIready established by the metabolfe gradient of the cell. If the combined negativities of these two sources establishes a threshold electrotonic current the cell will fire continuously at the arbitrary frequency indicated in the lower record. (Geseld, Blood. Heart and Circulation. Lancaster, Pa.: Science Press 1940.)

of breathing. In this search we have invoked the implications of the neurohistological descriptions of Rayon y Cajal (26), Bartelemez (56), Lorente DE No (57), BodIAN (58) and others, according to which nerve cells are literally covered with an almost continuous layer of hundreds or even thousands of tiny boutons. These are the synapses for fibers converging on the cells.

Since the signals impinging on any single neuron must be temporally scattered, without a ghost of a system, the orderly rhythmical outflow of impulses at the axon hillock would seem to demand a new and special mechanism of transfer of influence from one nerve cell to another. Highly doubtful, as we believe it is, that dendrites and nerve cell bodies conduct impulses in the sense that nerve cells do, we have adopted an entirely new outlook. Each impulse impinging on the neuromembrane is pictured as producing a local negativity in the immediate region of the exciting bouton. Each local negativity adds its tiny quota to the existing inherent electrotonic current in the 
manner suggested in fig. 22. If the sum of the inherent and reflexogenic electrotonic current is below threshold value the cell will fail to fire regardless of the number of signals impinging on the cell. If it increases above that value it will fire with increasing frequency as the current increases with increasing impingement of signals. Should the signals straggle the discharge will again diminish.

Such an hypothesis calls for no precise requirements of temporal sequence of stimuli demanded by the prevailing theories of synaptic transmission.

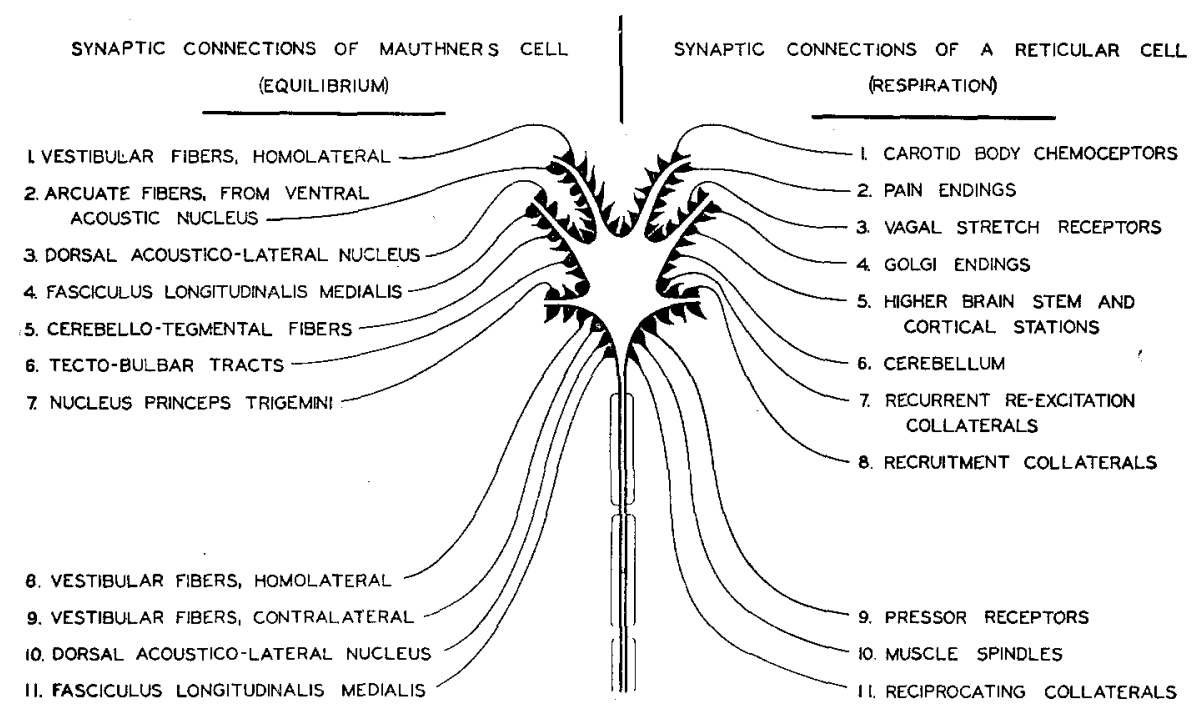

Fig. 23. A comparison of the heterogeneous convergence of signals in MAUTHNER's cells of the fish and in the reticular cells of the respiratory center. The outflow of but one type of signal along the neuraxons of the pair of MAUTHNER's cells which are produced by a heterogeneous and a temporal group of signals is regarded as evidence against temporal theories of synaptic transmission and in favor of the theory of mass drive. The extent of active surface unrelated to such arrangemet of synaptic scales becomes the determining factor. (GEselL: Heart, Blood and Circulation. Lancaster, Pa.: Science Press 1940.)

Mechanisms of temporal organization, for which there is no evidence, are not essential. Quantity of current arising from the effects of scattered signals of heterogenous sources is the underlying principle. The thoroughly studied MAUThNer cells will illustrate our way of thinking more clearly [BARTELmez (56)] (see fig. 23, 32 and 33). These remarkable cells, of which there are only two in a single individual, control the equilibrium of the fish. They are gigantic, visible to the naked eye, and are provided with thousands of synapses conceivably bringing as many as a million signals per second to each cell. These signals arrive in a variety of 12 groups of fibers all related to motor equilibrium impinge upon a common neuromembrane which through a common response to any negativity regardless of its origin creates but one new type of signal. These leave each cell for all levels of the cord by one neuraxon only.

These two cells must be regarded as a center. For simplicity, they are unexcelled in revealing the machinery of convergence. They imply a common 
action of impingement of a large volume of heterogenous signals which according to older views is supposed to work by some miraculous temporal arrangement. There can be little opportunity of a selective use of signals as suggested by the writings of Lorente de No. Each cell would seem, rather to act in the simple fashion of a funnel or adding machine. The faster the inflow of water or figures the greater the outflow or sum. It is with that in mind that we have arranged a diagram comparing the simple center composed of two MaUthners cells with the more diffuse respiratory center of reticular cells in the medulla (see fig. 23). Each reticular cell is assumed to receive its drive from a comparably large variety of fibers: 1. chemoceptors, 2. pain endings, 3. Gouar endings, 4. muscle spindles, 5. proprioceptive endings of the lungs, 6 . pressoreceptors of the carotid sinus and aortic arch, 7. veno-pressor receptors at the heart, 8. recurrent reexcitation collaterals, 9. recruitment collaterals, 10. reciprocating collaterals and others. That a miscellaneous group of signals such as those arriving by these fibers could function on a temporal mechanism seems beyond all possibility, despite continued attempts of interpretation in that direction. For reasons such as these and others to follow, we have substituted the theory of synaptic drive for that of synaptic transmission.

\section{After-Discharge.}

The continued activity of a nerve cell after cessation of stimulation of its converging fibers has been a well recognized phenomenon for many years. Perhaps the most familiar example is the continued inhibition of the heart outlasting faradic stimulation of the peripheral end of the vagus. After-discharge has been repeatedly described in the higher integrations by SHERRINGTON and his colleagues [Creed, Denny-Brown, Ecches, Liddeld and Sierrington (59). To quote from BARD $(60)$ :

"This characteristic is found in almost all reflexes. When a single shock applied to an afferent nerve elicits a motor response, the mechanical and electrical records of the contraction reveal repetitive discharges over the motor neurons. Since the stimulus does not set up more than one impulse in each afferent fiber, it follows that the motor neurons continue to discharge impulses for some time after the single volley of afferent impulses has entered the cord. In the case of the flexor-reflex this after-discharge, as it is called, may last $100 \mathrm{msec}$. or longer. It is so pronounced in the crossed extensor reflex that mere observation of the limb movement suffices for its detection. In the flexor reflex it is not due to the contracting muscle reexciting itself reflexly through stimulation of proprioceptors by its own contraction, for it is not affected by deafferenting the muscle by section of the appropriate dorsal roots. In the crossed extensor reflex it is partly due to a proprioceptive autogenous excitation. We must conclude, therefore, that after-discharge is largely the result of a prolonged excitatory conditions somewhere along the central pathway."

The after-discharge following reflex stimulation of the scratch-reflex in fig. 24 may serve as a typical example [Sherrinaton (61)]. It will be seen that the stronger the stimulation the greater is the after-discharge. The discharges of sympathic ganglia persisting for a minute or more after the end 
of preganglionic stimulation [BRonk (62), LARABEE and Bronk (68)] illustrates the phenomenon in a much simpler preparation. Here too duration and intensity of the after-discharge vary in proportion to the duration and
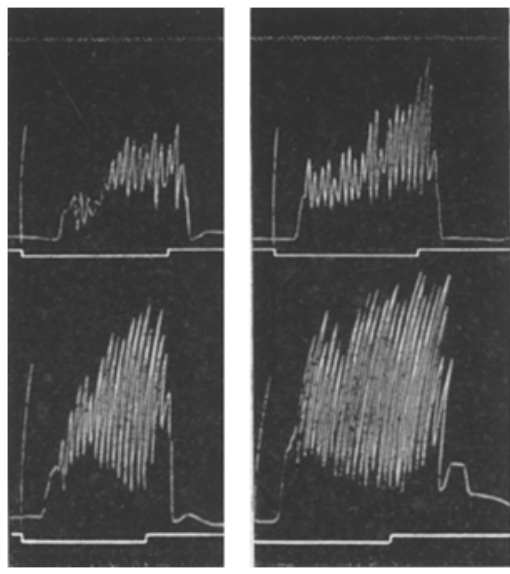

A
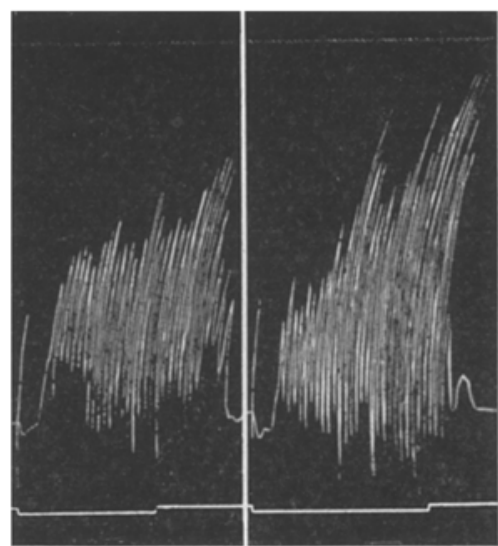

$\mathrm{B}$

Fig. 24. "A and B. Scratch-reflex graded by intensity of stimulus. The reflexes were elicited by unipolar faradization with needle as kathode to shoulder skin. Double shocks at rate of 17 per second. were used. The examples are taken from a series of 12 , exhibiting twelve grades of amplitude. The twelve grades of intensity of stimulus used were, reckoned in units of KRoNECKER inductorium scale, $250,350,475,690$, $1001,1900,3000,4000,5200,6300,7500$. The examples in the figure were the second, fourth, sixth, eighth, tenth, and twelfth of the series. Time above in fifths of seconds. The time of applieation of the stimulation is shown on the signal line below. Intervals of one minute elapsed between commencement of each reflex. The needle-kathode remained unmoved throughout," (SHERRINGron: Integrative Action of the Nervous System. New York: Charles Scribner's 1906.)

intensity of the preganglionic excitation. In like manner respiratory reflexes outlast electrical stimulation of all types of sensory nerves. After-stoppage of breathing from vagal stimulation and after-acceleration from stimulation of
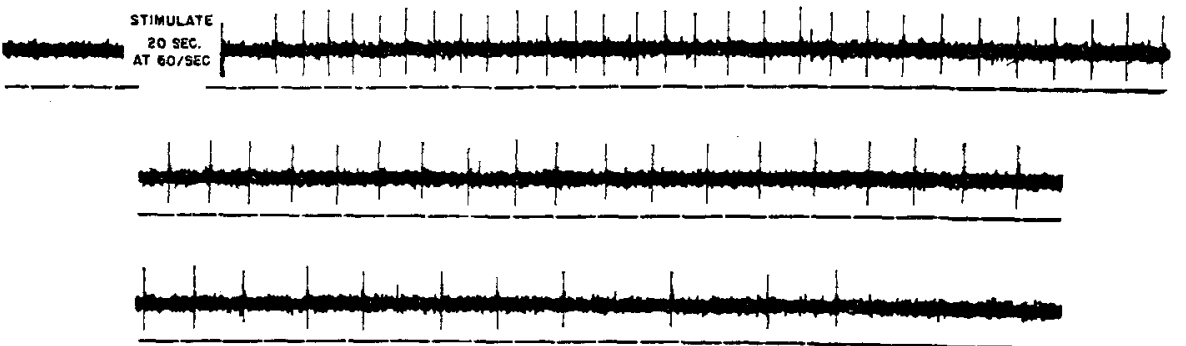

Fig. 25. Repetitive after-discharge from a ganglion cell following 20 sec. of preganglionic stimulation at 60 per sec. Continuous record. Last impulse 27 sec. after end of stimulation. Time: 0,5 sec. [LARABEE and Broxk: Proc. Soc. exper. a. Biol. a. Med. 38, 921 (1938).]

the saphenous nerve are readily denuonstrable. The phenomenon need not necessarily be considered abnormal for it is observed to occur during physiological stimulation as well. For example the inspiratory discharges cut off poorly during hypooxic hyperpnea as compared with eupnea and straggle on into the expiratory phase of breathing (see fig. 172). Since such hyperpnea 
is a purely reflexogenic response arising in the chemoceptors the continuation of inspiratory activity following the main inspiratory discharge may be regarded as an after-discharge of the inspiratory half center. In conformity with this interpretation it is found that faradic stimulation of the central end of the Hertngs nerve produces hyperpneas which outlast stimulation in proportion to the strength and duration of the current employed (Geseli, Hamruton and Brassfielo (64)] (fig. 26). These particular results show the extent of the after-discharge following a moderate stimulation. The decreased hydrogen ion concentration of the blood persisting into the period of hyperventilation,

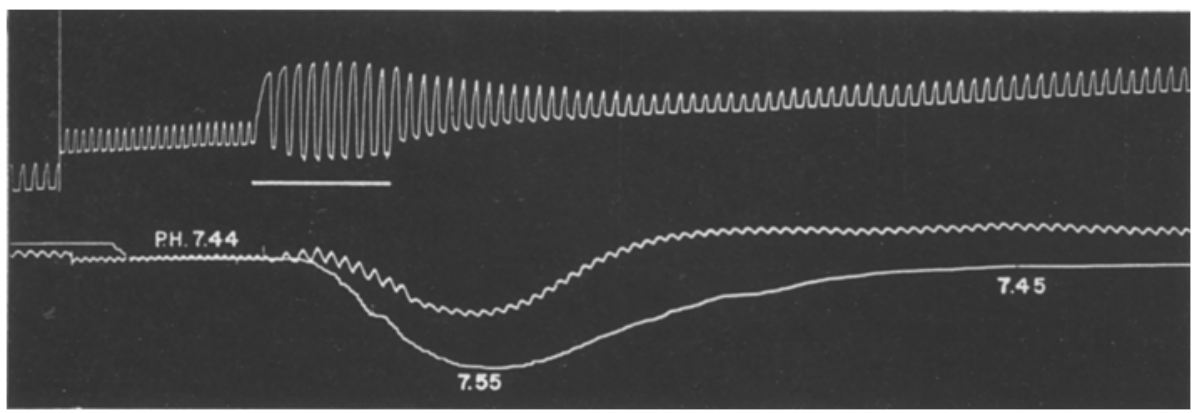

Fig. 26. Continuation of hyperpnea after cessátion of faradic stimulation of Merras nerve despite a

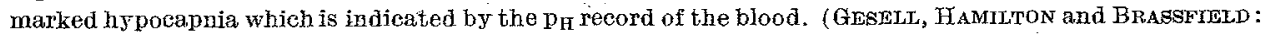
Unpublished.)

acting alone, should have produced apnea. The results, therefore, permit no questioning of the nature of the phenomenon. The short period of subnormal breathing shows that the after-discharge disappeared before the blood had returned to normal acidity.

It is clear that the phenomenon of after-discharge must be included in a complete understanding of respiratory control or for that matter of nervous integration in general. We are rather inclined to regard it as a naturally occurring phenomenon of considerable importance. But on that point there seems to be room for difference of opinion. To quote from Bronk:

"Because it (after-discharge) seldom appears following frequencies (of stimulation) of less than 50 per second it must be considered a clue to the nature of synaptic processes and not as a phenomenon associated with a naturally activated ganglion."

In harmony with our theory of electrotonic activation of nerve cell discharge we see distinct advantages to the processes underlying the phenomenon of after-discharge, for it is quantity of signals irrespective of temporal arrangement and, therefore, quantity of electrotonic excitation current that counts. For that reason an extension of the effects of a single synaptic discharge gives that discharge a longer driving action. Where coordination is of a low order, as in outlying ganglia, after-action of a signal on the neuromembrane permits the cell to operate with the aid of a smaller number of 
synapses. For simplicity of explanation we employ a nerve cell with but a single synapse such as illustrated in fig. 27,8 . The synapse, known as the calyx type, is exceptionally large and covers much of the excitatory pole opposite the axon hillock. The calyx synapse is of special interest because it excludes all possibility of convergence and, therefore, challenges the statement commonly made that a single synapse or single impulse will not fire a nerve cell. Undoubtedly the calyx synapse must have been overlooked in this connection. The large active area created by the arrival of a single impulse, theoretically at least may be expected to fire the cell, for LIt.LIE has established in the automatically discharging nerve cell model that the frequency of discharge is directly proportional to the area of the active metal exposed to the acid, or the amount of current flowing.

If it be assumed that the activity of the neuromembrane underlying the calyx synapse be of short duration, we may expect a corresponding short lived potential drop between the excitatory pole of the cell and the axon hillock. This is indicated by the spikes labelled ,synapse discharge" (fig. 28). As a result, each synaptic discharge would" produce but one discharge of the axon hillock of the receiving cell as indicated in the lower record labeled axon hillock discharge. That discharge would occur the moment the potential drop reached threshold value. Since the short lived electrotonic current subsides below threshold level during the refractory period of the axon hillock a second discharge could not occur. But if the activity of the neuromembrane beneath the synapse diminishes more slowly, as indicated in fig. 28-2 the cell would discharge so long as the electrotonic current continues above the threshold strength, at a frequency largely determined by the refractory period of the axon hillock. Lesser prolongation of synaptic activity such as represented in 28-3 could not increase the frequency of discharge above that in 28-1 unless the synaptic discharges were accelerated as shown in 28-4. Then the electrotonic current of the receiving cell would remain above threshold value and fire the axon hillock at a higher frequency. The rate of discharge would bear no relation to the incoming signals however. If next we assume that the frequency of axon hillock discharge is capable of varying with the magnitude of the electrotonic current an irregular synaptic discharge would tend to produce an irregularly changing potential drop and therefore an irregular discharge of the axon hillock (see fig. 28-5). In cells supplied with a dense covering of synapses, irregularity would be less probable due to the smoothing out effects of multitudinous local negativities. Fig. 28-6 illustrates our conception of true after-discharge. When the synapses stop firing the post electrotonic current subsides and the frequency of discharge retards and disappears the moment the subthreshold level is reached. In conclusion it may be suggested that after-action at the synapse will be found related to magnitude of impingement of signals on the cell, that the greater the number 


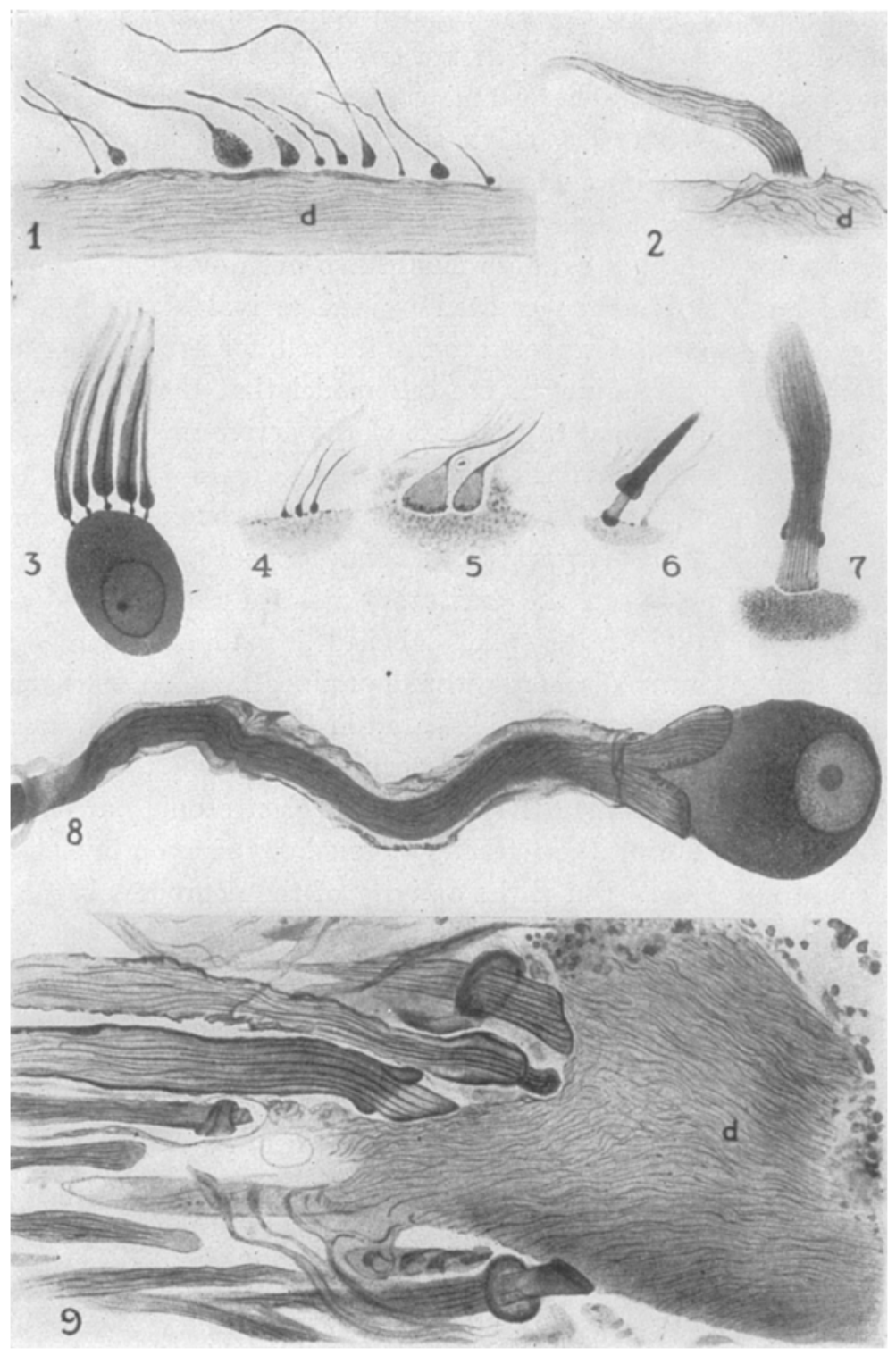

Fig. 27. Varieties of synapses of partionlar interest in relation to the discnssion of syaaptic after-action and nerve cell after-discharge and to the principle of surface active area. (See text page 515 . Note tremendous differences in the size of boutons.)

"Figures 1 to 9 represent types of synaptic endings found in acustico-lateral centers of goldfish; all stained with activated protargol method and drawn at $\times 1440$. Fig. 3 to 9 countelstained with Malloryazan; fig. $3,4,5,6,8$ and 9 from specimen 37 .

1. Large and small endfeet of HJLD-AUERBach on the ventral dendrite (d) of MatuThNers cell. Specimen 27.

2 Club ending of restibular fiber ending on lateral dendrite (d) of Mauriners cell. Note arrangement. of neurofibrils in the two synaptic elements. Specimen 21. Photomicrograph of same club in fig. 21.

3 fine myelinated fiber's ending on cell of medial acustic mucleus by means of tiny endfeet.

4 Fine unmyelinated fibers ending on perikaryon of MavrHners cell by means of tiny endfeet similan to those of fig. 3. Compare with four smallest endings in fig. 1. 
The Recurrent Collateral as a Challenge to the Theory of Synaptic Transmission. 517

of synapses impinging on a single neuron the less sustaining need their afteraction be to insure a regularity of cellular discharge. In this light the phenomenon assumes significance as a stabilizing influence in nervous integration.
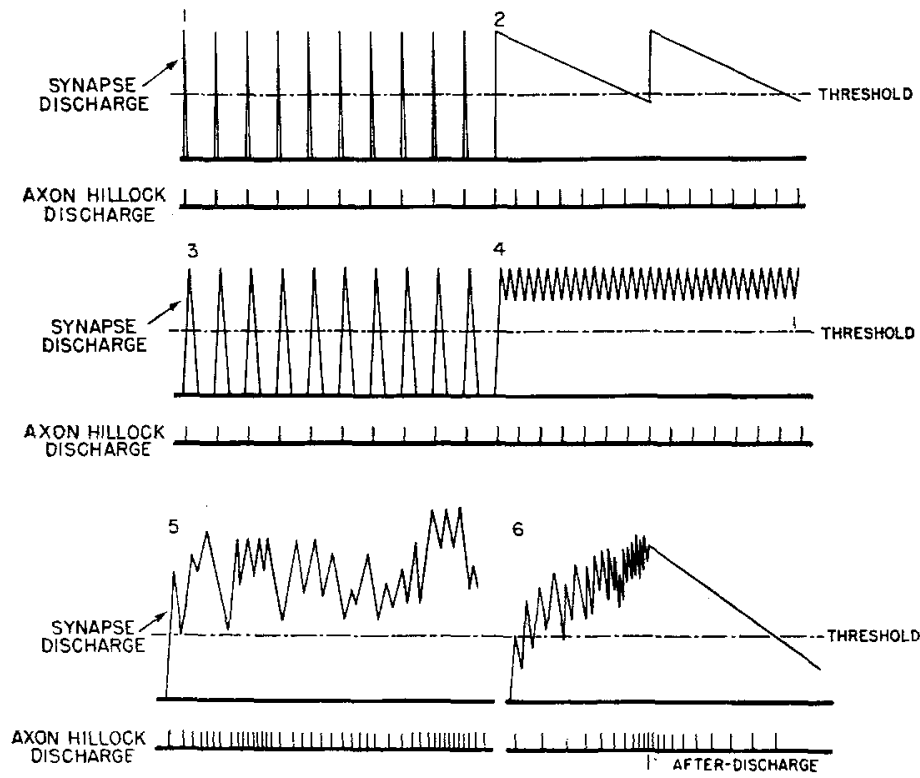

Fig. 28. Hypothetical schema of the physiological function of synaptic after-action and of neuron afterdischarge. The conditions required by this schema is a neuron supplied with but a single synapse of the calyx type. The impingement of a single impulse is assumed to be sufficient to discharge the secondary neuron. The horizontal line, marked threshold, indicates the intensity of the electrotonic current required to activate the cell. The spikes, labeled synapse discharge, indicate the momentary fluctuation of the electrotonic current produced by the impingement of a single impulse. The axon hillock discharge shows the frequency of firing of the secondary neuron. This is seen to vary with the duration of synaptic after-action and with the frequency of synaptic discharge. For further details consult text.

\section{The Recurrent Collateral as a Challenge to the Theory of Synaptic Transmission.}

The phenomenon of after-discharge should have seriously questioned the classical views of synaptic conduction, for if there was no evidence that the synapse itself continued to fire after the nerve fiber had delivered its signal, how could the continued activity of the nerve cell possibly be explained on a highly adjusted temporal arrangement? Vague as the whole subject was,

5 Large endfeet on ventral dendrite of MAUTHNERs cell. Note band of redstained granular mitochondria at the terminal surfaces of the endfeet. Compare with largest endfeet in fig. 1, 18 and 20.

6 Club-like ending of small myelinated fiber ending on perikaryon of MAUTHNERs cell. Note band of mitochondria at terminal surface of ending and myelin sheath (red) investing axon.

$7 \mathrm{Club}$ ending of large myelinated fiber ending on lateral dendrite of MavTrNers cell. Neurofibrils in dendrite are cut transversely and appear as minute dots. Compare with fig. 2 , in which myelin sheath and mitochondria at terminal surface of club are not stained. Specimen 35 .

8 Large myelinated vestibular fiber (left) forming a calyciform ending, emabracing portion of tangential nucleus cell (right). Note band of granular mitochondria at terminal surface of calyz, and myelin sheath (red) enveloping axon (left).

9 Several large myelinated vestibular fibers ending by means of clubshaped terminals on lateral dendrite (d) of MAUTHNERs cell. Note arrangement of neurofibrils in axons and in dendrite, and the difference in their caliber. Dendrite cut obliquely." [Bodian; J. comp. Neur. 68, 117 (1937).] 
the firmly implanted idea that nerve cells functioned by conducting impulses seemed to stand in the way of a new conception, for are not effects actually conducted from the peripheral receptors through the central nervous system to the end organs? But effects seemed to be confused with impulses for if the question is examined critically what evidence is there of the conduction of an impulse from one neuron to another when convergence of any marked degree occurs. Yet this is the present status of the subject as recently summarized in the Synaptic Symposium by Gasser (65), Erlanger (66), Bronk (62) Lorente de No (67) and Forbes (68). Not only are impulses believed to travel along the dendrites and cell body to the neuraxon but antidromically from the neuraxon to the dendrites [Eccles and Hoff (69)], Gasser (70), Creed, Denny Brown, Eccles and Sherrington (71)]. And on this pure assumption precise quantitative experiments are performed and basic conclusions drawn concerning the nature of the process of synaptic transmission.

The difficulty of a direct approach to the problem is realized by all. On the assumption that the physiology of the synapse is but a sample of neurophysiology, Lucas was among the first to consider an intensive study of the neuraxon as the practical method of approach [see Fonbes (68)], Gasser (65) has taken the same point of view as indicated in the opening paper of the symposium on Synaptic Transmission.

"The first question that would naturally be asked about the synapse is: What is the nature of the material coming in contact at its borders? The answer to the question must come from a direct study of the synaptio region of the neuron, and for that reason it is a difficult one to obtain. As an introduction to the problem, attention has been directed to properties of the axon, because of the expectation that the events which take place in those parts of the neuron entering into the synapse may resemble, qualitatively at least, events taking place in other parts of the neuron. If it could be shown that the expectation has a foundation in fact, to the excent to which it holds, axon physiology could be transferred directly to synapse physiology."

LoRfNTE DE No (67) comments as follows:

"The transmission of impulses through any neuron is not an event that necessarily occurs after the activation of any synapse on the neuron. On the contrary, it is an event that fails to take place unless several synapses are activated, and, moreover, unless the activation complies with a set of most rigid and exacting conditions. Figuratively speaking, it may be said that synaptic transmission is 'optional' for any neuron; therefore, without detailed knowledge of the anatomical and physiological conditions that determine the 'choice', it cannot be predicted whether stimulation of fibers of a certain nerve, or of a certain pathway, will result in transmission; nor can it be predicted through which anatomical channels the transmission, if at all, will be effected." He states further that "The soma of the motor neuron is electrically excitable and the nerve impulse when entering it is accompanied by an electrical sign and that the impulse is locally initiated under the most densely activated zone of the synaptic scale, and that from there on it spreads over the remainder of the soma and also enters the axon."

The barriers to a direct approach to the problem have been most effective but sometimes nature gives the clue where direct experiments seem almost hopeless. It is on this very point that the recurrent collateral seems to bear in a most critical way. These most interesting structures which have puzzled physiologists are described and illustrated by CAJAL (26) in three rarieties 
The Recurrent Collateral as a Challenge to the Theory of Synaptic Transmission. 519

of cells- the anterior horn, the Purkinje and pyramidal (see fig. 29 and 30 and schema 31). Assume for example that signals from converging fibers $A, B$

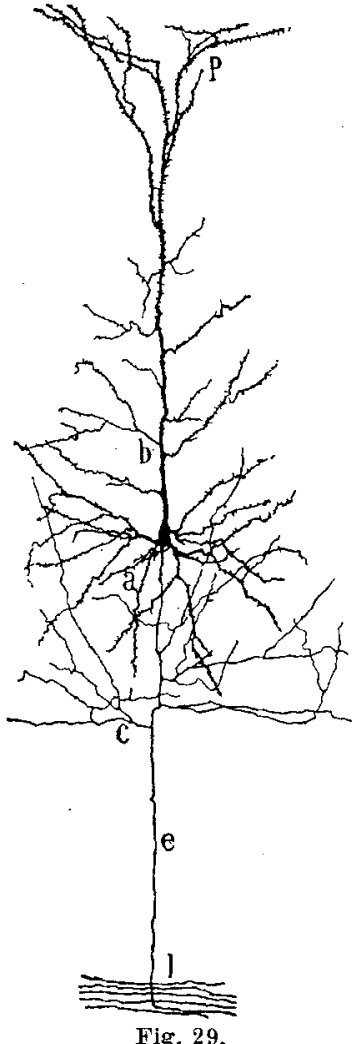

Fig. 29

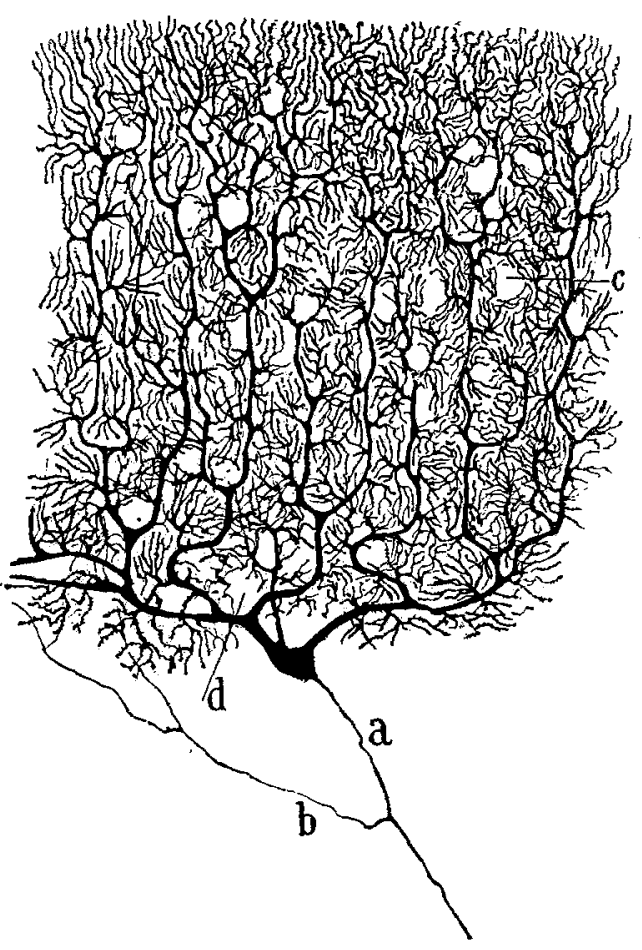

Fig. 30.

Fig. 29 and 30. Pyramidal and Purkinje cells showing recurrent collaterals. (RaMon y CAJAL: Histologie du système Nerveux, Vol. 1. Paris: A. Meloine 1909.)

and $\mathrm{C}$ in fig. 31 have set up an excitatory wave in the nerve cell which then passes down the perikaryon in the accepted way. On reaching the point $E$ the impulse travels back through the recurrent collateral to $\mathrm{D}$, which had just been activated. The time required for the impulse to travel the microscopic distance DED must be infinitesimally small, meaning that the returning signal would find $\mathrm{D}$ in the refractory period and, therefore, unresponsive. If on the other hand we assume that synaptic activation of the neuromembrane is a relatively local phenomenon dependent upon

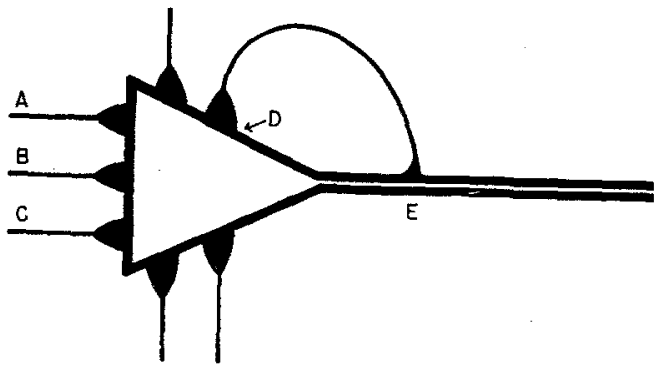

Fig. 31. Schema indicating that an impulse passing point $D$ on its way to the neuraxon would on returning via the recurrent collateral find point $D$ refractory. Query- How do such implications conform with the theories of synaptic transmission and synaptic drive? the recent arrival of a signal, locus $D$ will be responsive to recurring signals for if these discharges are of a relatively low frequency, which is usually the 
case, $D$ will respond to every recurring signal and thereby increase the general potential drop.

We, therefore, like to think of each signal returning at $D$ as reestablishing activity and, therefore, adding its quota of negativity at that point. That effect could be attained by a pure electrical excitation, by increasing the local metabolic rate, by a change of permeability or most probably, as we prefer to interpret the function of the neuron, by a deposition of acetylcholine. Such a deposition of acetylcholine would be comparable to the local application of chloroform or $\mathrm{KCl}$ in the Nitella [Osternout and HrLu (45)] which was seen to produce a rhythmic discharge of its covering membrane. The purpose which the recurrent collaterals serve is a most interesting speculation. All indications point to an automatic mechanism of self excitation which could be employed either as a preventive against lowered activity consequent upon fatigue or for increasing the activity and the development of fatigue thereby providing a cutting of mechanism for the production of rhythmic activity such as the respiratory center displays.

In conclusion we should like to add a pertinent comment by Forres (72) in his review of spinal reflexes written in 1922, for its bearing on our concept of limitation of synaptic action at the neuromembrane. "In the spinal cat with proper controls for electrode polarization the flexion reflex may show marked fatigue in a half-minute or so. That the fatigue is central is proved by the fact that immediately after failure of reflex response stimulation of the motor nerve will evoke a full-sized contraction of the muscle."

"Sherringtor and Lee and Everingham have shown that such reflex fatigue does not involve the motor neuron as a whole, but only the particular channel of approach to it which has been employed. Under Sherringtons direction I found that fatigue of the flexion reflex as evoked through the popliteal nerve usually did not impair the response evoked immediately afterwards through the peroneal nerve, and vice versa. Indeed, the test reflex evoked through one nerve was usually even more vigorous after fatigue of the reflex arch through the other nerve than before, although when the fatiguing stimuli were above a certain strength the subsequent test reflex showed slight impairment. SherRington concluded from evidence of this sort that the seat of fatigue is in the particular synapse whereby the motor neurone is excited."

\section{An Electrotonic Theory of Nerve Cell Inhibition.}

Should excitation solve itself in terms of increased neurocellular potential drop, inhibition must conversely solve itself into a corresponding decrease of potential drop. By allocating the boutons transmitting inhibitory signals to a region in the immediate proximity of the axon hillock (see fig. 23, 46 and 58 ), such an arrangement is readily consummated. This region then becomes 
electrically negative in response to "inhibitory" signals as the excitatory end of the cell does to its "excitatory" signals. The negativity thus imposed by the inhibitory signals diminishes the cellular potential drop from the dendrites to the neuroaxon and opposes the outflow of current at the axon hillock. Should this current fall below the threshold value the cell would stop discharging. Thus excitation and inhibition become fundamentally the same phenomena.

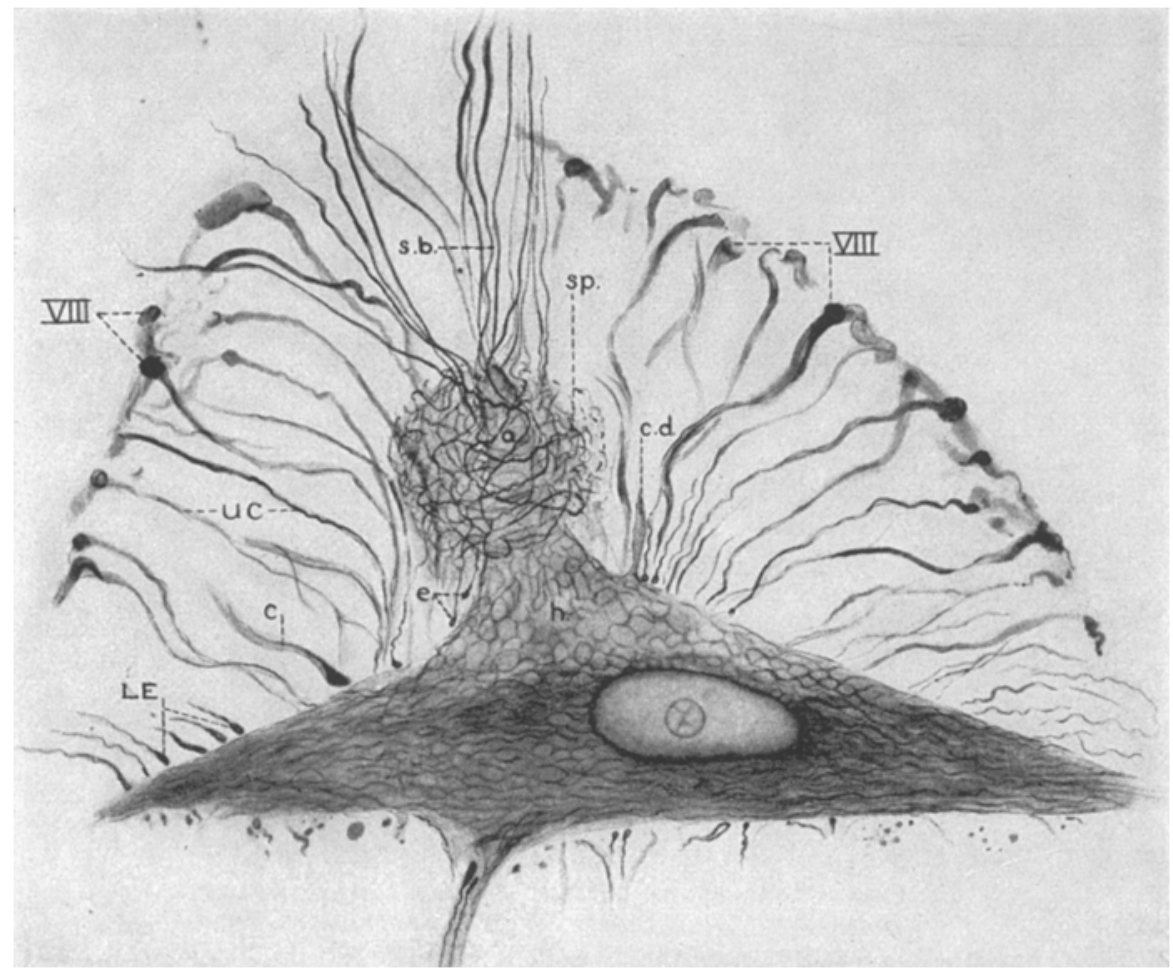

Fig. 3\%. 'Perikaryon of MAUTHNER's cell showing details of axon cap as they appear in a selective nerve fiber impregnation with activated protargol. Note irregular spiral of fine fibers forming the centra] core of the axon cap (sp.). These fibers enter the spiral as a bundle (s. b.) which arises in the supracommis. sural part of the fasciculus longitudinalis medialis, and leave the spiral to terminate on the axom hillock by means of tiny endfeet (3). Also note the unmyelinated collaterals (U.C.) which arise from VIII fibers and radiate toward the axon hillock around which they terminate bluntly (see fig. 83 ), or by meaus of endieet. L. . ., large endfeet; c., myelinated club ending. Other legends as in fig. 33. Specimen $17 . \mathrm{X} .720$," [BODIAN: J. comp. Neur. 68, 117 (1937).]

The effects of excitatory and inhibitory signals would differ only as a result of their geographical impingement upon the cell.

But this control of potential drop which we have outined is not merely speculation. Again Mautunea's cells assume the greatest interest and importance for these truly remarkable cells give support to the electrotonic theory of inbibition as they do to electrotonic excitation. A very conspicuous group of fibers advancing in special formation, winding about the axon hillock and ending in a mass of tiny boutons has excited the curiosity of neurohistologists [see fig. 32 and 33 by Booras (58)]. On a purely structural basis this 
arrangement suggested a localization of function in the nerve cell, excitation in the dendritic end and inhibition on the axon hillock end.

The strategic position of these hypothetical inhibitory boutons at the axon hillock is interesting in that an impressed negativity at a point of convergence where the current leaves the cell must be very effective. The extremely small size of the inhibitory boutons (see fig. 33) fortunately allows room for

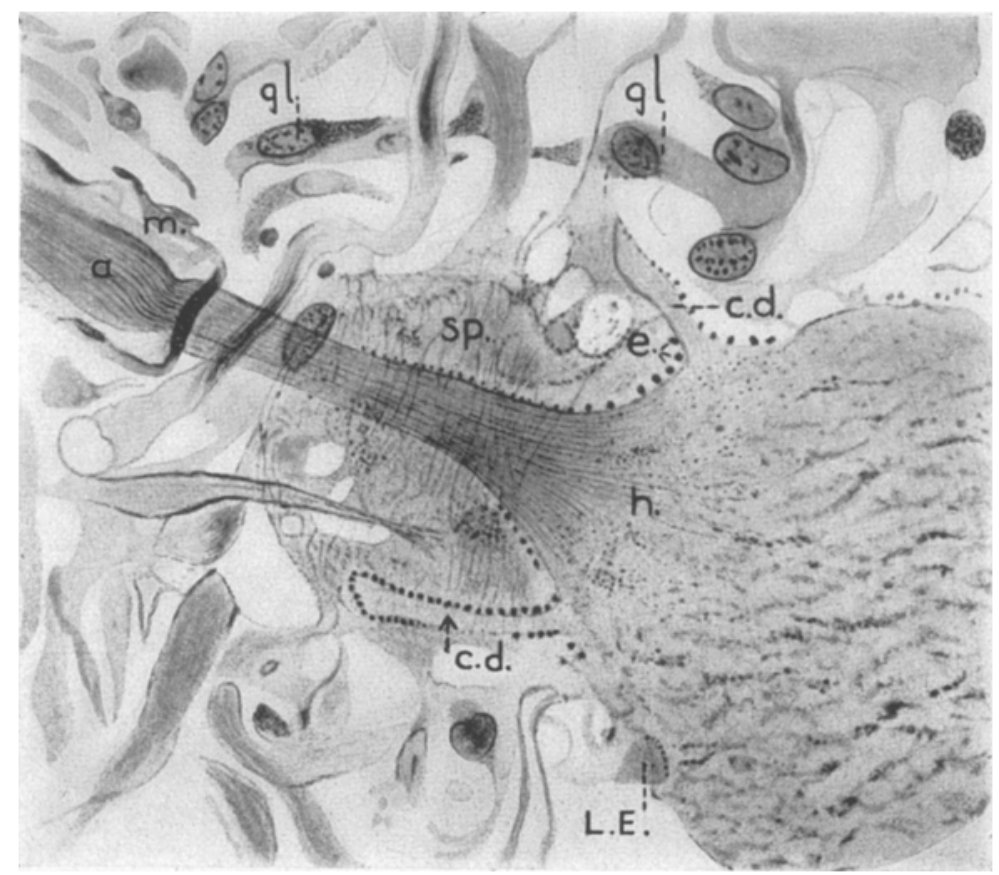

Fig. 33. "Perikaryon ofMaUTHNeR's cell, showing axon hillock (b), axon (a), central portion of axon cap (sp), and tiny terminal feet (e) of fine unmylinated fibers in central part of axon cap. m., beginning of myelin sheath of Mavtaners fiber; gl., glia; c. d., cap dendrite; L. E., large endfoot. Specimen 37 . X 1440." [Bodian: J. comp. Neur, 68, 117 (1937).]

the impingement of a large number of boutons in the limited region of the axon hillock. This would seem essential for a highly graded inhibition. Looking for analogous arrangements in higher forms the basket-like connection around the body of the Purkinje cells assume a new interest (see fig. 34 by CAJAL (26)]. Until histological evidence is found, pro or con, it will be well to bear in mind that the dendrites represent by far the greatest portion of the active surface area of a neuron, therefore an alternative arrangement of connections is possible in which the inhibitory boutons might be stationed at the base of each dendrite instead of at the axon hillock. Highly concentrated increased negativities at these restricted areas would block the descending currents in each dendrite and thereby the total outflow of current at the axon hillock.

For simplicity of representation in our schemas we have connected all such fibers as are believed to be excitatory to the excitatory pole of the reticular 
cell and such fibers as may possibly be inhibitory to the inhibitory pole (see fig. 46).

The question may well be asked how can an impressed negativity at the axon hillock produce inhibition without causing excitation of the neuraxon beyond? The answer is given arbitrarily in our schema (fig. 35) where a threshold stimulus of $10 \mathrm{M} . \mathrm{V}$. is assumed to be necessary to fire the axon hillock (see Section A) and a similar potential drop between the firing hillock and number 1 node of RANVIER is assumed to be necessary to fire the neuraxon proper. The continuous voltage drop is assumed to fire the hillock at a frequency of 10 per second. Each increase of 1. M.V. potential drop between the dendrites and axon hillock, let us say from peripheral chemoceptor signals, will add one extra discharge per second. So at 20 M.V. potential drop the rate would increase to 20 per second (see Section B). It is obvious that this acce-

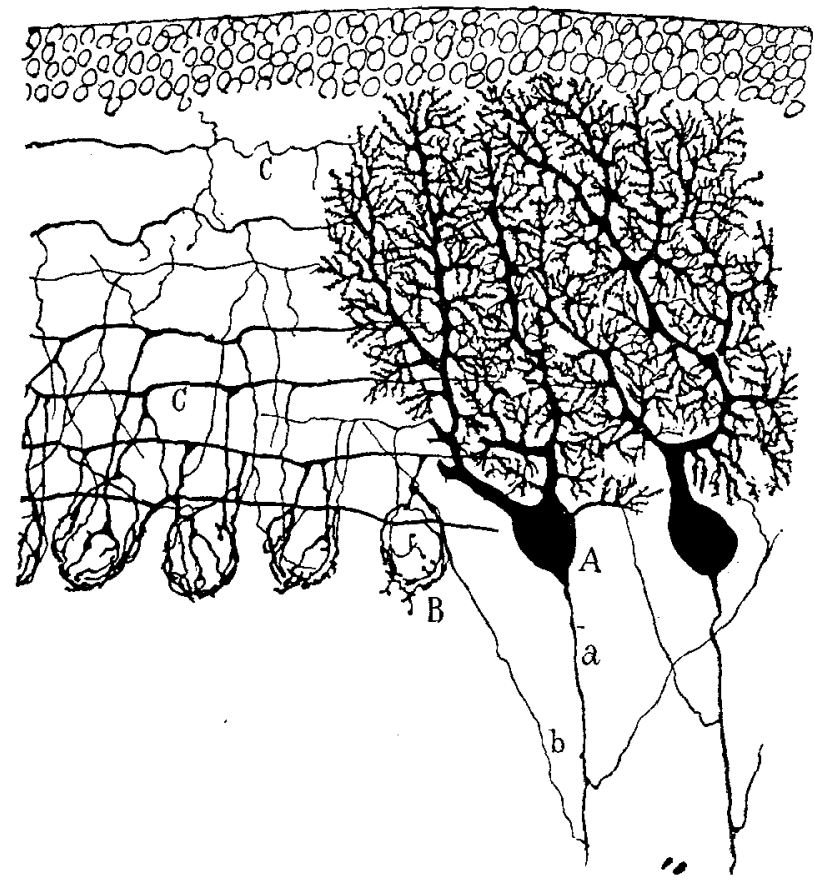

Fig. 34. Basket connections about cell bodies of PURKINJE cell. (Ramox y CaJaL: Histologie du système Nervenx, Vol.1, p. 735. Paris: A. Meloine 1909.) lerated rate of firing might be reduced in two ways, by withdrawing reflexogenic drive from the dendritic end or by increasing the negativity at the axon hillock. A reduction of $15 \mathrm{M} . \mathrm{V}$. of reflexogenic drive from the $20 \mathrm{M} . \mathrm{V}$. potential drop to $5 \mathrm{M} . \mathrm{V}$. would for example produce a stimulation deficit of $5 \mathrm{M} . \mathrm{V}$. or an inhibitory asset of the same value. This would need to be overcome before reexcitation could occur. 'Or if we begin with a lower potential drop of $10 \mathrm{M}$.V. the same results would be accomplished by impressing a steady negativity of $5 \mathrm{M} . \mathrm{V}$. at the axon hillock through the inhibitory boutons (see Section C). Here, as in the first example, the cellular potential drop would be 5 M.V. below par and, therefore, the axon hillock would not fire. Sustained negativity at the axon hillock would also establish an electrotonic current between the axon hillock and node \# 1 with a potential drop of 5 M.V. which would be $5 \mathrm{M} . \mathrm{V}$. short of the amount required to fire the neuraxon. Another and much simpler conception meeting the requirements of inhibition is to assume that the potential gradient required to fire the axon hillock is low as 


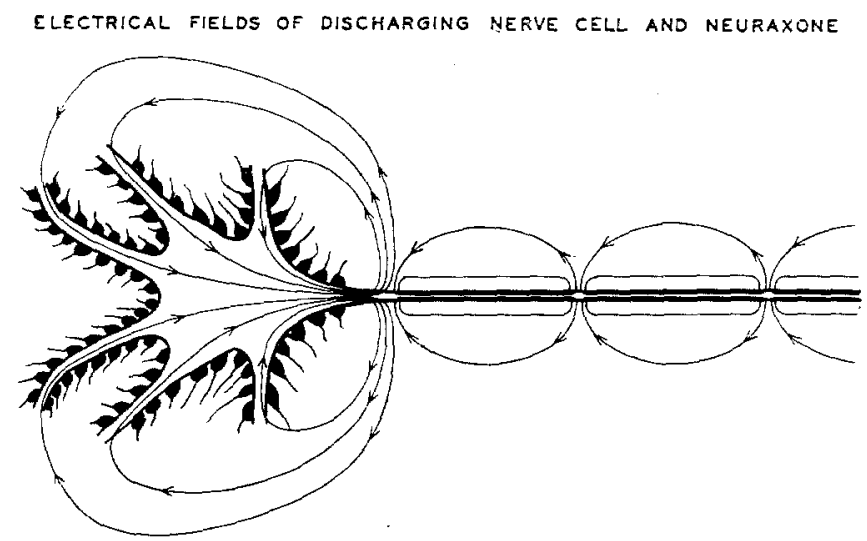

POTENTIAL DROPS OF DISCHARgING NERVE CELL AND NEURAXONE

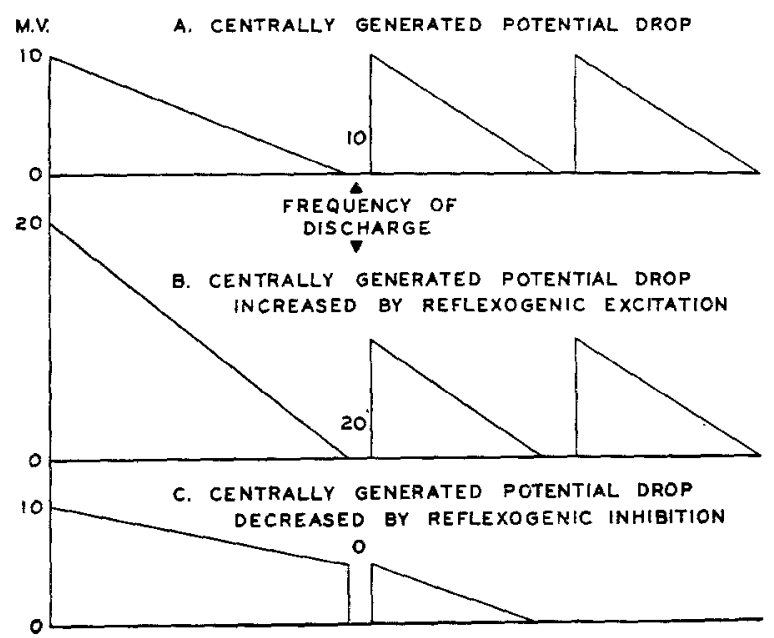

Fig. 35. Schema illustrating the electrotonic mechanism of inhibition. See text for details.

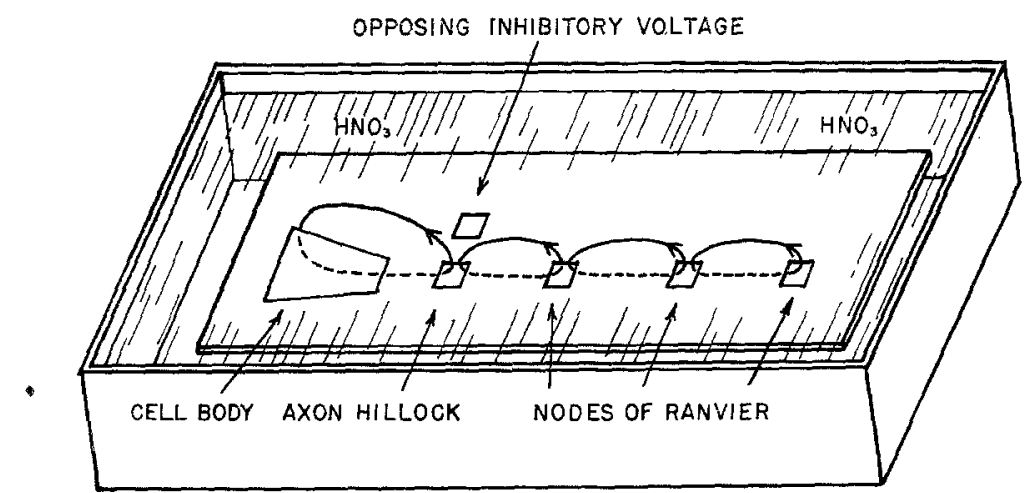

Fig. 36. Inorganic model demonstrating that approximation of voltage opposing the outflow of currel.t. at the "axon hillock" will retard or stop the rhythmic discharges from the "nerve cell". The applicaticn of such inhibitory voltage fails to stimulate the "neuraxon" beyond.

compared with that required to fire the neuraxon. This would allow a relatively high continuing negativity at the axon hillock without the danger of discharging. the neuraxon. 
This arbitrary explanation of inhibition is supported by our inorganic nerve model (fig. 36) (GEsell, Unpublished). A plate of Armco iron is covered with paraffin except for the areas indicated "cell body", "axon hillock" and "nodes of Ranvier". When such a model is placed in nitric acid the activity of the large area of exposed metal corresponding to the cell body and its dendrites periodically excites the axon hillock. Each discharge of the axon hillock is then propogated with the greatest velocity from node to node as postulated by LiLlire. Just as Liluie showed that the amount of active area and, therefore, the amount of current flowing, determines the frequency of firing we have shown that suppression of this current by approximating an opposing voltage will retard or stop the discharge of the axon hillock completely without setting up new discharges in the neighboring "nodes of Ranvier".

\section{An Hypothetical Structural Basis of Respiratory Activity Patterns.}

Assuming that the continuing discharge of the reticular cells is the basic activity upon which the respiratory act is built, it becomes desirable to de-

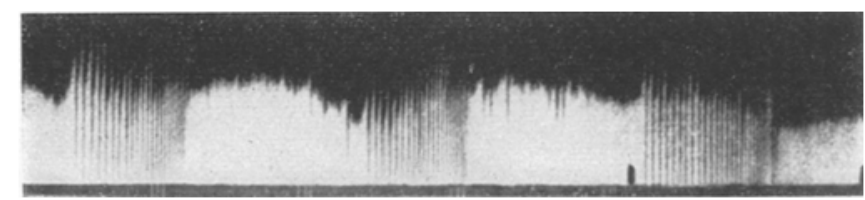

Fig. 37. Rlythmically grouped discharges of the isolated nerve fiber automatically developing in response to the current of injury at the cut end of the nerve. [ADRIAN: Proc. roy. Soc. Lond. 106 (B), 596 (1930)].

termine the mechanism for periodically accelerating and interrupting this train of discharges. We have already shown that some central mechanism exists which is capable of responding in a rhythmical way to the steady chemical drives of centrogenic and reflexogenic origin. This was established in the curare experiments in which periodic signals were completely eliminated. Such a central mechanism is generally thought to reside in the reticular formation of the medulla. But the nature of the slowly augmenting acceleration and recruitment of the inspiratory contraction must still be determined.

Adprians (46) records (see fig. 37) of the response of the isolated nerve fiber to its own current of injury shows groupings of impulses of diminishing frequency, indicating a decreasing ability of the nerve fiber to respond to a steady flow of current. Gasser (78 and 74) has shown the importance of previous activity of nerve fibers in the production of rhythmic activity by a superimposed induction shock upon a continuing discharge (see fig. 38). Grouped discharges may actually come to an end as a result of sheer exhaustion of the nerve and its failure to respond to prevailing stimulation. In this respect the isolated nerve, may be no different from the respiratory center for such has 
been the theory in regard to breathing. 'The end of an inspiratory discharge in a vagotomized animal in which the so-called inhibitory reflexes are eliminated

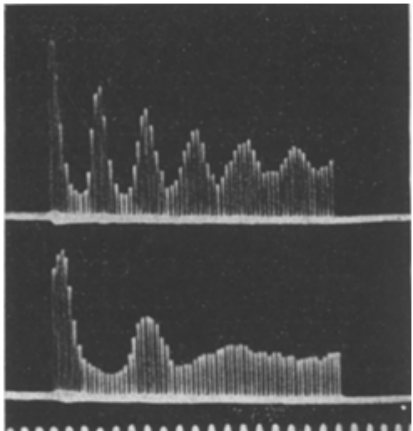

Fig. 38. Periodicity superimposed upon a discharging nerve fiber by a single induction shock. (GAsser: Harvey Lecture) 1936. is assumed to occur when the center has spent itself.

To these chemical mechanisms of interruption we have added an hypothetical structural mechanism which gives some faint promise of explaining not only interruption but acceleration of frequency discharge as well. I refer to the recurrent collateral whose function is still a matter of pure speculation. The idea is best followed with the help of figures 39 and 40 . The recurrent collaterals are pictured as carrying signals back to the dendrites of the cell, thereby increasing the sum total of signals impinging on the cell. The resulting increased cellular potential drop increases the frequency of the axon hillock discharge. The increasing recurrent signals in turn increase the potential drop still more which increases the rate of discharge and the number of recurrent signals and so on. The
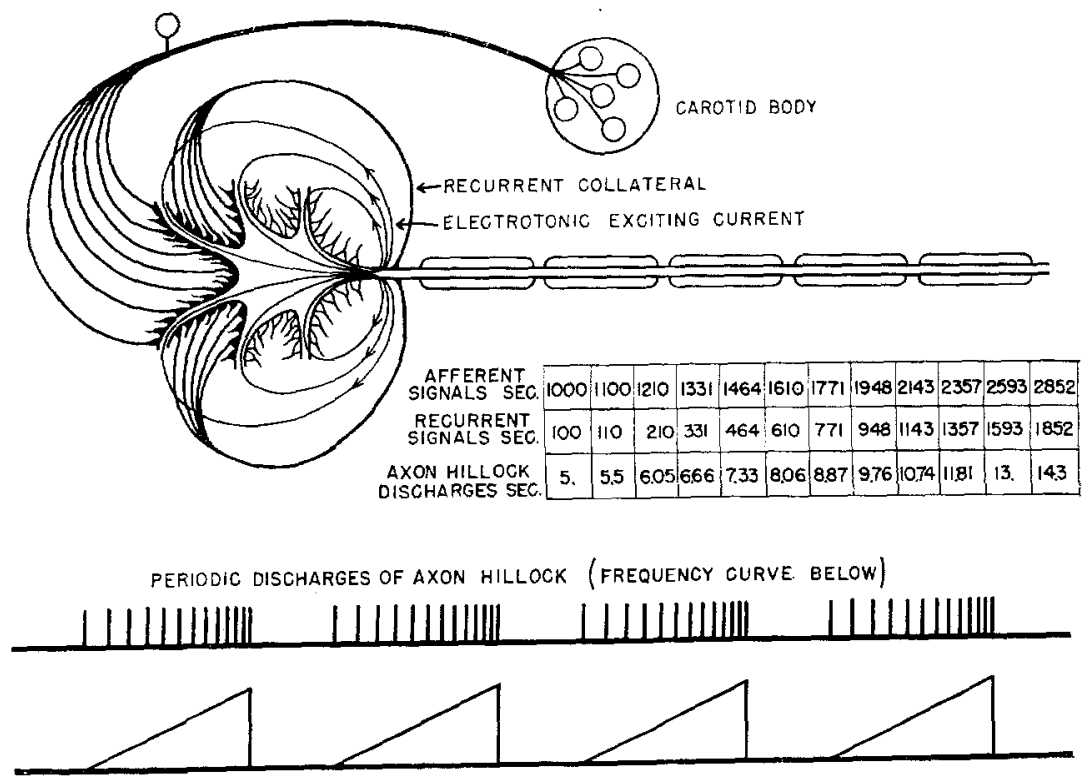

Fig. 39. An hypothetical mechanism of rhythmical acceleration and interruption of the steady state discharge. Recurrent collaterals carry signals from the neuraxon back to the excitatory end of the neuron thereby increasing the electrotonic excitation current up to the end of the inspiratory discharge. See text for further explanation. (GEsels: Heart, Blood and Circulation. 1940.)

progressive nature of the self augmenting cycle is shown schematically in fig. 39. This effect cannot continue indefinitely. Its very nature implies a self limitation, set by the peculiar properties of the neuromembrane which is 
firing at the axon hillock. The ability of the cell body to meet the increasing needs of repair must play a most important role. Progressively increasing activity raises the threshold of excitation at a rate disproportionate to the increasing electrotonic current due to growing exhaustion (see fig. 40). Increasing electrotonic current fails to maintain its lead. It crosses under the more abruptly rising curve of threshold of excitation and the cell stops firing at once. The reexcitation component which had been built to high values, therefore, suddenly weakens which delays further firing until the threshold

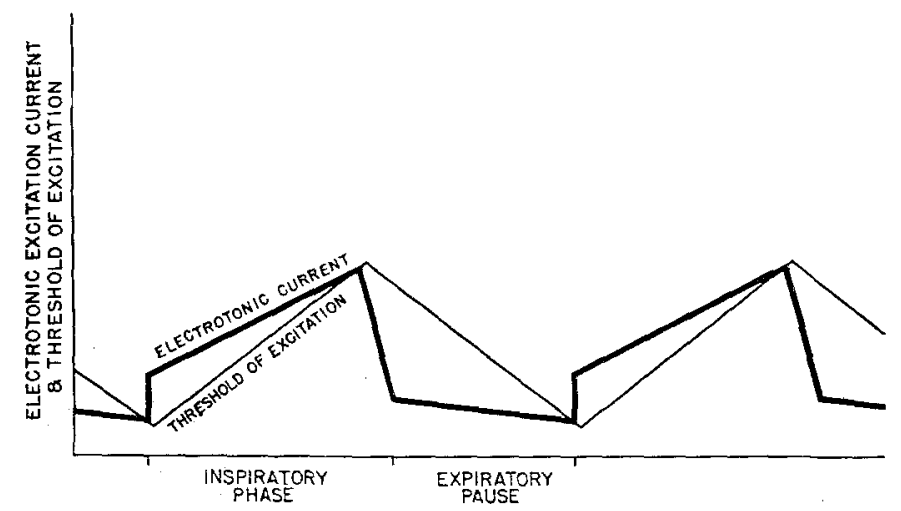

Fig. 40. Graph showing the hypothetical changes in the electrotonic excitation current and the threshold of excitation ereating acceleration of frequency and intermption of the steady state diseharge. See text. [Atkinson, Brown and Gegell: Amer. J. Physiol. 129, 303 (1940).]

of excitability falls below the more slowing diminishing electrotonic current. The cell then fires and the cycle repeats itself again.

\section{A Central Neurostructural Basis for Recruitment.}

Acceleration of frequency is but the smaller part of increasing muscle power in the slowly augmenting type of contraction. Recruitment of additional elements, in our experience, is by all odds the more important of the two. Though separate phenomena, acceleration and recruitment are inextricable, for acceleration of one or more reticular cells may be the deciding factor for the recruitment of another. What then is a simple mechanism of recruitment? Highly sensitive reticular cells are placed at the head of the reticular columns to act as pace setters for the group (see fig. 41). These cells may be assumed to possess a high potential gradient and a responsive neuromembrane. Slight differences in sensitivity may possibly start the inspiratory fusillade now in one cell and now in another. The stream of signals once started, let us say in cell \#1, descends in a reticulo-spinal neuraxon to its group of anterior horn cells. On its way, this stream supplies signals by collaterals to the excitatory pole of cell \#2 which is still quiescent, but near the threshold of activity. In due time, as the collateral signals from cell \#1 take effect, cell \#2 begins to fire. Cell \#2 then sets off cell \# 3 in a similar way. Recruitment 


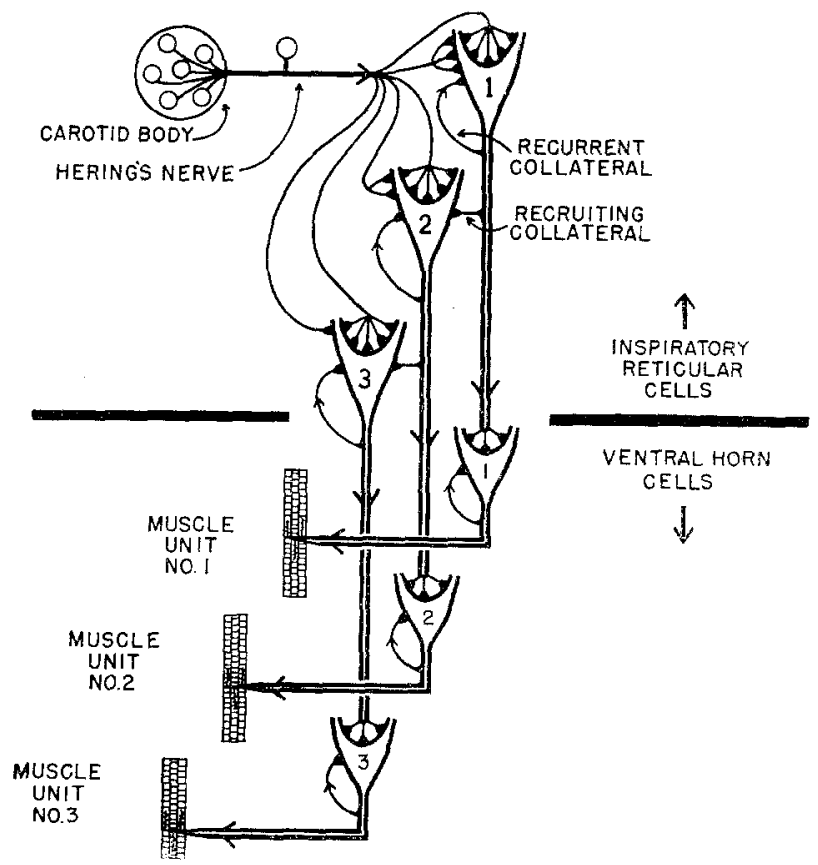

Fig. 41. An hypothetical mechanism of recruitment in the inspiratory half center dependent upon seria! connections of the reticular cells with recruiting collaterals. Note hypothetical gradation of impingement of chemoceptor signals on inspiratory reticular cells. (Eight synaptic connections from the chemoceptors have been allotted to reticular cell \# 1 , seven to cell $\# 2$ and six to cell $\# 3$.) Such an arrangement would assist in maintaining the pace setters at the head of the reticular column. Recruiting collaterals were omitted on the expiratory half center (see fig. 43 and 46) to conform with the steady state pattern of activity. Graded impingement of chemoceptor fibers in the expiratory center would however be suitable for adding new active expiratory units with increasing chemical stimulation, but that has been omitted

in the diagram. For further details see text: (GrSELI: Heart, Blood and Circulation. 1940.)

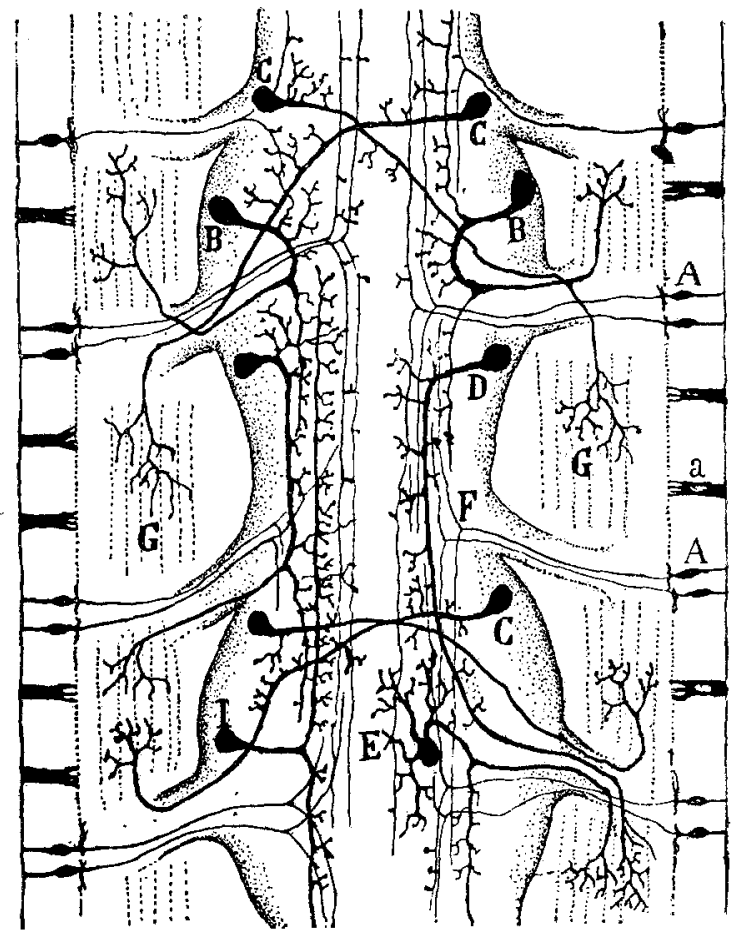

Fig. 42. Intermotor connections described by ReTzius and LENHOSSEK. (From RAMON $\bar{Y}$ CAJAL: Histologie du système Nerveux, Vol. 1. Paris: A. Meloine 1909.) 
thus continues down the reticular column until cell $\$ 1$ ceases to fire. Absence of signals from cell \# 1 withdraws heavy support from cell \# 2 and brings it to rest by reduction of its electrotonic current below threshold value. Cell \# 3 follows suit. Decruitment is more rapid than recruitment as might be expected from the sudden falling off of cell \#1. As anatomical basis of the mechanism of recruitment (fig. 42) is reproduced showing the purely intermotor connections of Retzrus and V. Lenhossex (26).

\section{Central Reciprocating Interaction of the Respiratory Half-Centers.}

It is customary to think of breathing as a balanced motor act in which inspiration and expiration transpire in closely regulated reciprocal relations even though expiratory activity in the muscles is sometime missing and often weak. Granting a total absence of contraction of the expiratory muscles the probability still remains that the expiratory half-center in the medulla is actually discharging, though not vigorously enough to fire the expiratory motor cells in the spinal cord. That being the case, the phasic shift which we are about to describe may always play a part in the integration of the respiratory act.

The mechanism we propose is this (see fig. 43). The pace setter (cell \#1) on the inspiratory side leads off. It provides signals by way of the reciprocating collaterals to the inhibitory poles of an antagonistic expiratory reticular cell which, let us say at that moment, is active. The result of such an arrangement is reciprocal inhibition. As inspiration waxes the inhibitory action on the opposing cell increases in proportion to the number of inspiratory signals impinging on the expiratory neuron. But that is only one-half of the mechanism, for the expiratory reticular neuraxon is connected by a collateral to the inhibitory pole of its inspiratory mate. As the expiratory reticalar cell is inhibited. and lowers its rate of firing it withdraws its restraint on inspiratory cell \# 1 . This is tantamount to excitation, for it is irrelevant, as we have seen, whether the inhibitory pole turns less negative or the excitatory pole more negative. Inspiratory cells thus draw their own excitation from their opposing cells. This should be a most positive arrangement of reciprocating activity. Conceivably it could be a highly important adjunct to hyperpnea for when the expiratory muscles are very active quick reciprocal shifts in activity are imperative. As we shall see it seems to be part and parcel of the phenomenon of rebound.

This mechanism of central reciprocating activity is very different from the reflex reciprocal inhibition originally proposed by SHenRInGton (61). It is more like that advanced by Brown (75 and 76) for rhythmic activity of the cord. Our mechanism however is more inclusive in that it recognizes reciprocal activation as well as reciprocal inhibition and presents specific means by 
which the reciprocating activities may be accomplished. Our concept of reciprocal inhibition differs more fundamentally from that advanced by Gasser (73). His view calls for dual afferent connections with internuncial neurons plus a synchronizing mechanism for shifting the afferent excitation alternately from the flexor to the extensor neuron. In our opinion the requirement of a

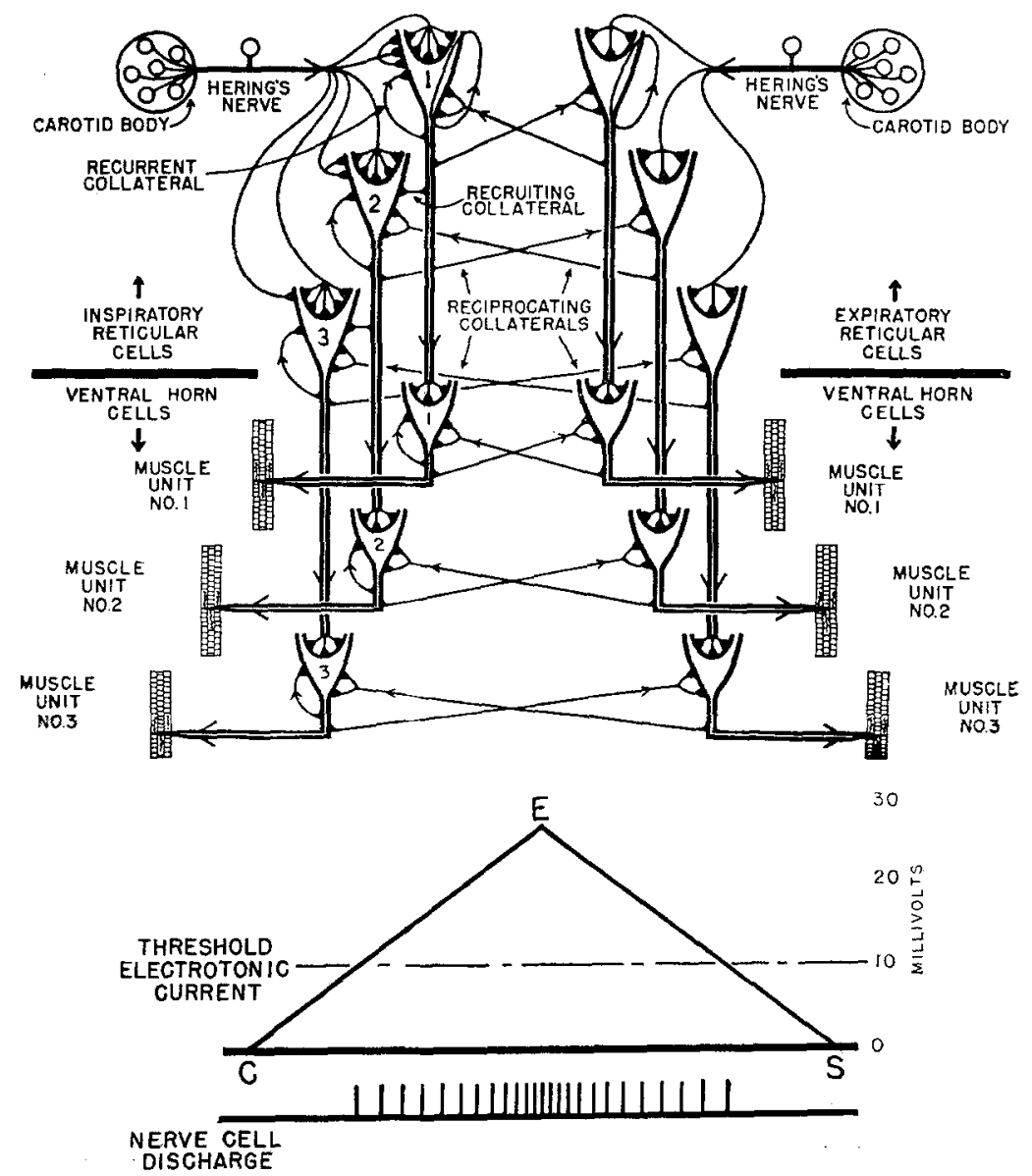

Fig. 43. Schema showing hypothetical connections for recrutment and for reciprocal inhibition and excitation between half-centers. Note that reciprocating connections are assumed to exist both in the center and cord. It is not illogical to assume recruiting collaterals in the cord as well as in the center. Fcr fuller discussion see text. (Gesell: Heart, Blood and Circulation. 1940.)

synchronizing mechanism eliminates his theory as a possibility. At least we, like Wyss (93), have been unable to discover the existence of this phenomenon described by Adrian and Bronts (6) and Rijlant (8) either in muscle or nerve centers.

Our concept raises a still more important issue, namely, is direct reflex inhibition of motor integrations an established phenomenon of major importance? To us it seems infinitely more simple and more certain to look upon motor reflexes as positively driven activities. A flexion reflex elicited during the course of an extensor thrust would, in that light, not produce a direct reflex 
inhibition of the extensors but working through the reciprocating connections of the half-centers would set up an indirect or reciprocal inhibition of the flexor reflex. Only under very special conditions, to be described below, are we able to reconcile a direct or non-reciprocal inhibition with our views of motor integration.

\section{The Central Excitatory State and the Central Inhibitory State.}

Much has been written on the central excitatory state and central inhibitory state. They are without question the outstanding phenomena of nervous integration yet little is known of their fundamental nature. To quote from the selected writings of Sir Charles Sherrington, central excitatory state is but a convenient.

"Name given to an enduring condition set up by a centripetal volley in the reflex centre. The experimental evidence for its existence is as follows:

(a) When separated by certain intervals (usually not longer than $20 \sigma$ ), two centripetal volleys (either in the same or different afferent nerves) evoke a reflex discharge from motoneurones which do not respond to either volley alone (Sherrington, Breimer, ECCles and Sherrington).

(b) When two centripetal volleys are separated by certain intervals (usually not longer than $20 \sigma$ ), the central reflex-time of the response to the second volley is greatly shortened (even to less than $0,5 \sigma$ ) (Eccles and Sherrington).

Both these experiments show that an excitatory condition set up by the first centripetal volley in the reflex "centre" persiste until the arrival of the second volley. On account of this property of existing for some time, this central excitatory condition is called the central excitatory state, c. e.s. The experimental observations (a) and (b) also show that for some motoneurones the c. e. s. produced by one centripetal volley sums with that produced by another volley. Since this happens under the most varied conditions, it can be concluded that in any motoneurones there is a summation of the c.e.s. produced by individual centripetal impulses which are separated by a sufficiently short interval." [SHeRrington (77)].

Inhibition is analysed in much the same way as is excitation.

"A single inhibitory afferent volley was pitted against a single excitatory volley of known strength, at different intervals of time and in different strengths. Its effect was also analysed in relation to an antidromic volley. The reflex tested was the flexion induced by stimulation of an afferent nerve in the same limb, inhibited by stimulation of the corrosponding afferent in the opposite limb (spinal cat). The inhibitory influence on the motoneurone is called the central inbibitory state ('c.i.s.')." [ShERRTnGTon (77)].

In relation to our views, expressed above, on the role of direct reflex inhibition it is pertinent to suggest that inhibition so studied is of central reciprocal origin i. e. a homolateral flexion reflex elicited by reflex excitation of the homolateral flexor center is inhibited by a contralateral flexion reflex elicited by reflex excitation of the contrareflex center. No direct reflex inhibition would be involved.

C.E.S. and C.I.S. are compared by SherRINGToN and his associates in the following way.

"In many respects c.e.s. and c.i.s. are analogous states, but they differ fundamentally in certain features. In the following respects c.e.s. and c.i.s. are analogous: 
1. c.e.s. and c.i.s. are only produced when nerve impulses in the terminal branches of one neurone are incident on a neurone next in series-i.e., at synapses. However, there is yet no experimental evidence for the existence of inhibition with neurones other than motoneurones.

2. The c.e.s. or c.i.s. produced by a single impulse undergoes a gradual subsidence. With flexor motoneurones the rate of subsidence for c.e.s. (Ecchess and SHbrRINGton) is several times more rapid than the rate for c.i.s.

3. The c.e.s. or c.i.s. produced by one impulse sums respectively with the c.e.s. or c.i.s. produced by other impulses arriving by either the same or other nerve fibres ending on that same motoneurone. It may be that there is only summation between the c.e.s. or c.i.s. produced at immediately adjacent synapses.

4. As a consequence of summation many grades of intensity of either c.e.s. or c.i.s. may be produced on a motoneurone.

5. When c.e.s. and c.i.s. are opposed to each other, there is an inactivation of certain quantities of each. This mutual inactivation is likely to be quantitative, in which case there is a true algebraic summation between the c.e.s. and the c.i.s. of a motoneurone. It follows that c.e.s. and c.i.s. cannot coexist in the same place for any appreciable time. It may be that there is a quantitative interaction between the c.e.s. produced by one excitatory impulse and the c.i.s. produced by one inhibitory impulse, but such a relationship is at present pure hypothesis.

In the following respects c.e.s. and c.i.s. differ fundamentally:

I. If the c.e.s. of a motoneurone reaches a sufficient intensity, then it gives rise to the discharge of an impulse from that motoneurone. No corresponding action is known for c.is. It has no direct effect on the motoneurone-it melely inactivates c.e.s.

2. An impulse passing antidromically up a motor nerve fibre to a motoneurone inactivates the c.e.s. of that motoneurone, but does not affect the c.i.s." [SHaRRINGTON (77)].

According to Sherrington and his group:

"There are some differences of opinion about the nature of c.e.s. The extreme views are perhaps represented as follows:

1. That the electical responses of successive nerve-impulses summate, on analogy with the 'retention of action-current' of crustacean nerve, or the negative after-potential of vertebrate nerve.

2. That each nerve-impulse produces a quantum of exciting agent, a chemical substance, which sums with other quanta formed at the same or neighbouring points by other impulses.

The disappearance of c.e.s. after an antidromic impulse shows that c.e.s. must ai least be largely restricted to those parts of the motoneurone which are accessible to such an impulse i. e. to the surface membrane of the perikaryon and dendrites, for, in analogy to peripheral nerve, the impulse should traverse the surface membrane of the motoneurone. It does not seem possible that a chemical substance, such as is postulated in the second hypothesis, would be restricted to the surface membrane of the motoneurone or be inactivated by an antidromic impulse.

In criticism of the first hypothesis it may be pointed out that the negative after-potential of vertebrate nerve is possibly an abnormal condition of excised peripheral nerve resulting from absence of blood-supply and fatigue, as is shown by its close relation to the supernormal phase. Again, it is improbable that an antidromic impulse would diminish negative after-potential, but rather that it would add to the existing negative after-potential. There is another process in peripheral nerve which may give a clue to the nature of c.e.s., namely the local excitatory scate. It is an excitatory process losalized to the stimulated region of the excitable tissue, and it is capable of summation. Although very short in duration in peripheral nerve (about $1 \sigma$ at most), it is longer in other excitable tissues, e.g. it has a duration of at least $8 \sigma$ in heart-muscle. Moreover, the removal of local excitatory state at a point by the passage of a nerve-impulse is analogous to the disappearance of c.e.s. after an antidromic volley or a reflex discharge. Thus it seems likely the c.e.s. is a specialized manifestation of the local excitatory state. According to the membrane theory of nerve conduction the local excitatory state is a partial depolarization of the membrane surrounding the axis cylinders of nerve-fibres. By analogy, c.e.s. is probably a depolarization of those parts of the surface membranes of motoneurones on which 
excitatory impulses impinges, i.e. the synaptic membranes. It has been stated that some summation of the c.e.s. is produced by excitatory impulses reaching different synapses of the same motoneurone. The mechanism of this summation is not clear, but it must be remembered that the surface menabrane of the motoneurone is common to all its synapses, so that a change at any one synapse, e.g. due to the formation of c.e.s., could be accompanied by changes at other synapses." [Crhed, Denny-Brown, Ecoless, Liddelli and Sherrington (59)].

Although gradation of C.E.S. is readily demonstrable in the subliminal range it has not been recognized in the supraliminal range. It is held that C.E.S. exists only in the subliminal range for "present concepts indicate that it is not possible to have C.E.S. of supraliminal value. As soon as the threshold is reached, a discharge will set up with a consequent disappearance of C.F.S." [Creed, Denny-Brown, Eccles, Liddell and Sherrington (59)].

Our concept of C.E.S. and C.I.S. is based on our electrotonic theory of nerve cell activation and differs in several fundamental aspects from that of Sherrington. We propose that the electrotonic excitation current is in reality the central excitatory state. It, therefore, cannot be limited to the subliminal range but must extend from zero level to that of the strongest. excitatory current which obtains. Nor does our theory agree with the disappearance of C.E.S. each time a cell discharges. While it is probable that the electrotonic current would fluctuate with each cell discharge we picture a relatively uniform electrotonic current or C.E.S. during a uniform or steady state impingement of signals. The frequency of cellular discharge under such conditions would be determined by the duration of the refractory period of the axon hillock and by the intensity of the electrotonic current. Should the electrotonic current increase as a result of a greater impingement of impulses on the motoneurone the resulting local negativities at the base of each synapse would sum, irrespective of their geographical proximity to each other or independent of their temporal arrangement. There would be but one essential to nerve cell activity namely: a sufficient active neuromembrane surface to set up an electrotonic current of threshold value. Once the threshold electrotonic current is reached, the frequency of discharge will increase with the intensity of the electrotonic current or C.E.S. (see fig. 43A).

Such reasoning indicates why a conditioning centripetal volley should under satisfactory conditions virtually eliminate the latent period of the second volley. (It is cut "even to less than $0.5 \sigma$ " according to Eccues and Sherrington). For example (see fig. 43A) should the first "conditioning" centripetal volley bring the C.E.S. or electrotonic current of the motoneurone just under the threshold electrotonic current that cell would be on the very verge of firing. So when the second volley arrives virtually no time would be lost in eliciting a discharge (Fig. $43 \mathrm{~A}$ ).

On the basis of our electrotonic theory of "excitation" and "inhibition" there is every reason to expect the greatest similarity between C.E.S. and C.I.S. such as that described above by SHERRINGTon et al. As we have already 
suggested C.E.S. and C.I.S. are identical phenomena and differ only in geographical distribution. Effects of inhibitory impulse may, therefore, be expected to sum and subside at the axon hillock in the same way as do the effects of excitatory impulses at the excitatory end of the motoneurone. So too is a mutual quantitative inactivation to be expected wherever C.E.S. and C.I.S. interact. Reciprocating machinery ensuring such coordinated mutual inactivation has been discussed above.

Differences of C.E.S. and C.I.S. described by Sherrington may be more apparent than real. For example it is held that C.E.S. gives rise to a discharge and that no corresponding action is known for C.I.S. "It has no direct effect upon the motoneurone - it merely inactivates C.E.S." [SHERRINGTON (77)]. Our theory holds that "excitatory" impulses i.e. those impinging on the excitatory end of the motorneurone and the "inhibitory" impulses i.e. those impinging on the inhibitory end of the motorneurone produce local negativities or "neuromembrane activities" of a similar nature at the base of the synapse where they impinge. Furthermore, we have offered evidence in Section 23 for the view that a sudden withdrawal of inhibitory signals is capable of activating a nerve cell just as is a sudden arrival of excitatory signals. This concept is employed as an explanation of rebound, a most important phenomenon in the turning of the phase of any rhythmic activity such as breathing.

\section{The Dual Driving Action (Inspiratory and Expiratory) of the Chemoceptor Signals.}

With the aid of our hypothesis of reciprocating activity between halfcenters serving as a prelude, we may now return more specifically to the role of sensory impulses in motor integration. Reference to figures 41,43 and 46 will show that we have dispatched the impulses coming from the chemoceptors to the excitatory poles of both the inspiratory and expiratory reticular cells. The need of such dual connections is indicated by the selective excitatory action of cyanide on the inspiratory and expiratory act dependent upon the moment of its arrival at the carotid body [GESELL and Whiте "(78)] (see fig 44 and 45). Injected during the inspiratory phase it augments the inspiratory act which is then in progress (fig. 44). Reaching the carotid body during expiration it increases the expiration which is under way (fig. 45). It is thus concluded that both inspiratory and expiratory reticular cells are susceptible to reflexogenic drive of chemoceptive origin, that inspiratory and expiratory reticular cells are under continual bombardement from the outlying chemoceptors but that only one group is capable of firing at a time [Geselu and White (78)]. For further evidence of dual stimulation by the carotid body consult figures 26 and 88 , showing an increase in both inspiratory and expiratory activity as a result of electrical stimulation of Herisgs nerve. The relative weakness and scarcity 
The Dual Driving Action (Inspiratory and Expiratory) of the Chemoceptor Signals. 535 of eupneic expiratory contractions as compared with the inspiratory contractions revealed in our statistical analysis of potential patterns indicates

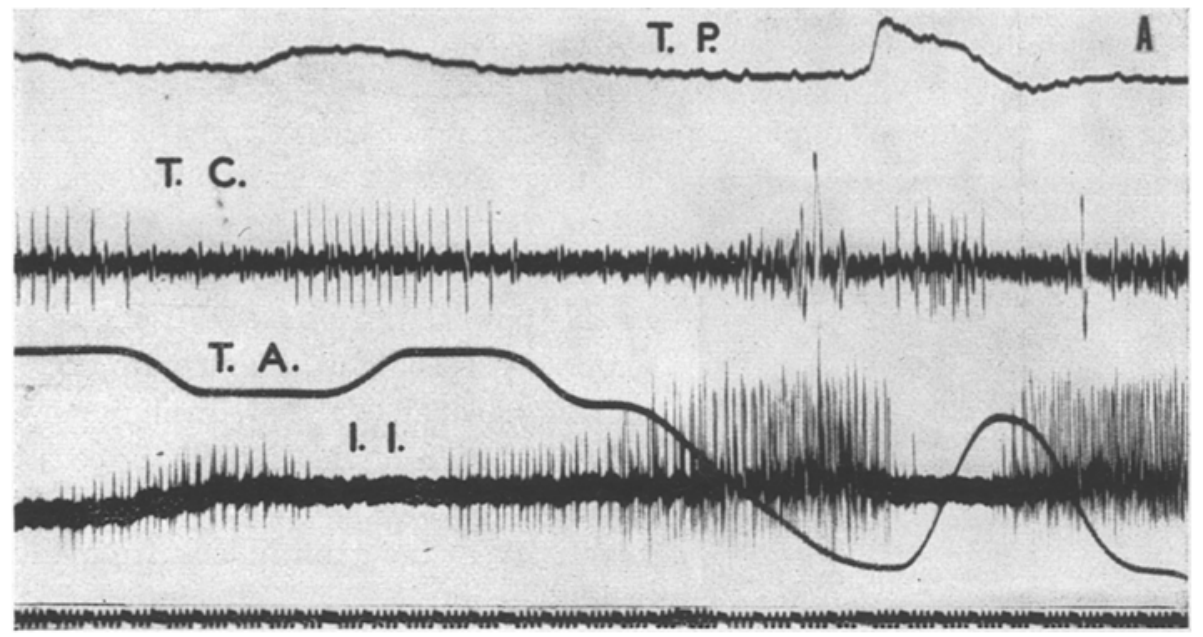

Fig. 44. Electrogram of the transcostar (an expiratory muscle) and the internal intercostal (an inspiratory muscle) showing intense activation of the internal intercostal when cyanide is injected into the carotid artery to reach the chemoceptors during the inspiratory phase of breathing. The inspiration which was in progress was markedly strengthened and prolonged. T. C. electrogram of transcostar muscle, I. I. electrogtam of internal intercostal muscle. T. P. tracheal pressure. T. A. tidal air. [GESELL and WHITE: Amer. J. Physiol. 122, 48 (1938).]

that relatively few chemoceptors connect with the expiratory reticular cells. On the other hand, the fact that expiratory contractions may be increased

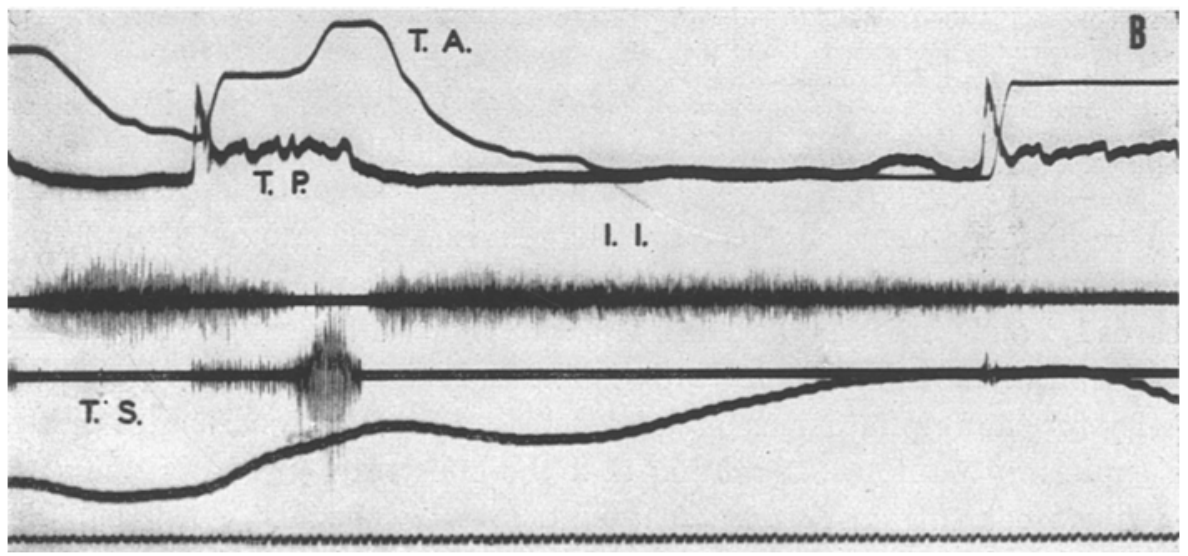

Fig.:45. Electrogram of the internal intercostal (an inspiratory muscle) and the transsterni (an expira. tory muscle) showing intensification of the expiratory act which was in progress when cyanide injected into the carotid artery reached the chemoceptors during the expiratory phase. The following inspixation is greatly prolonged into a typical appeusis due to the sustained chemical stimulation of the chemoceptors.

The vagus nerves were cut. [Geseicl and WHIt: Amer. J. Physiol. 122, 48 (1938).]

by $\mathrm{O}_{2}$ lack or $\mathrm{CO}_{2}$ excess at the chemoceptors indicates the possibilities of this drive in hyperpnea. 
At first thought, one might conclude that the stronger the dual peripheral chemical drive the greater will be the tendency for both sides to fire simultaneously but strangely enough the converse would seem to be the case. This rests on the efficiency of the reciprocating relations between "half-centers". The greater the activity of the two halves the greater becomes their reciprocating activity. For example, a powerful and rapidly developing inspiratory

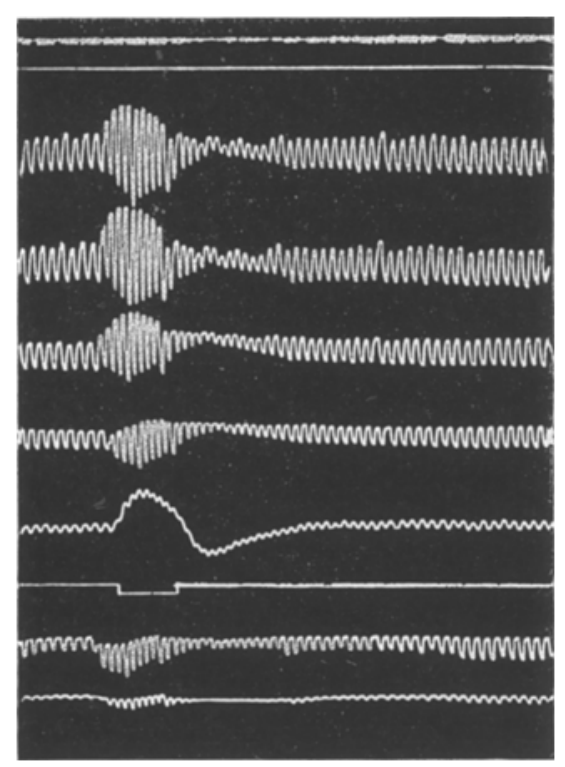

Fig. 46. Respiratory excursions of the chest and abdomen recorded with encircling bands showing increased inspiratory and expiratory activity on central faradic stimulation of the saphenous nerve. Upstroke indicates an expiratory excursion and downstroke an inspiratory excursion. [GESELL and MOYER: Quart. J. exper. Physiol. 25, 1 (1935).] discharge exerts a proportionately powerful and rapidly developing inhibitory effect upon the expiratory side. If at the onset of this powerful inspiratory discharge the expiratory cells are at a high state of activity they are capable of producing a powerful stimulation of the inspiratory cells by their sudden and effective withdrawal of negativity at the axon hillock from these cells. The result of such interaction should be an extremely effective shift from one phase of activity to the other.

\section{The Double Driving Action of the Nociceptor Signals.}

If it can be established that other sensory fibers impinge upon both halfcenters of respiration such as described for the chemoceptors, dual respiratory drive becomes a most important principle. It is, therefore, of interest to note the effects of faradie stimulation of the saphenous nerve upon respiratory movements of the dog (GEselu and Moyer, 79). Fig. 46 shows six respiratory records made with the aid of bands encircling the torso. Records 1, 2 and 3 are from the chest, 4, 5, 6 are from the abdomen. Upstroke indicates inspiration. Stimulation clearly increases both inspiratory and expiratory activity for the respiratory excursions increase beyond the normal inspiratory and expiratory positions. Granting that the effects are primarily due to the pain fibers coursing in cutaneo sensory nerves, it must be concluded, as it was for the chemoceptors, that the pain endings aro connected with both half centers of respiration. Pain fibers have accordingly been connected to both inspiratory and expiratory reticular cells in our schema (fig. 47). A continuous train of pain signals, like the chemoceptor signals, produces rhythmic breathing by virtue of the reciprocating interaction of half centers which allows excitation of but one half-center at a time to the exclusion of the other. The action of continuing trains of pain signals on breathing is no doubt similar 
to that upon rhythmic spinal reflexes. As is well known a flexion reflex set up by painful stimulation of the pad of the foot will often turn to rhythmic

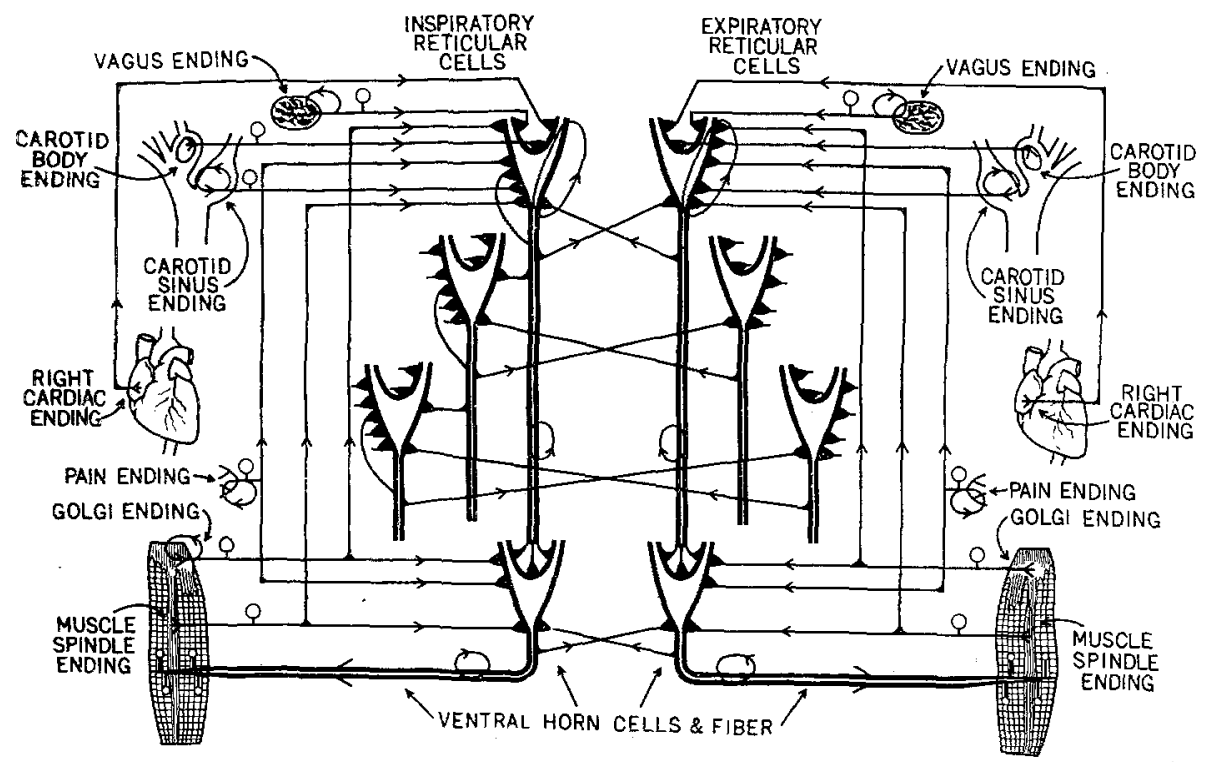

Fir. 47. Hypothetical summary of the integration of the respiratory act showing the major sensory motor and central connections. Excitatory afferents are connected to the excitatory poles of both inspiratory and expiratory reticular cells. Inhibitory afferents are connected to the inhibitory poles in both centers. Some afferents, such as those coming from the Gougr and muscle spindle endings are connected with the anterior horn cells as well. For a complete discussion see text. (GesELL: Heart, Blood and Circulation. 1940.)

stepping (see figure 77) [SHERRINToN (61)]. Continous stimulation provides the drive and the reciprocating mechanism provides the shift.

\section{The Reflexogenic Support of the Vagal Proprioceptive Signals.}

The profound effects of elimination of vagal function by vagotomy or block, the marked changes in breathing produced by artificial central stimulation of these nerves, and the modification of breathing produced by inflation or: deflation of the lungs leave no question of the importance of the vagus nerves in the control of breathing. This was generally recognized early in the 19 th Century as it is today, but the variability of results obtained with such simple procedures, then as now, leave many questions to be answered. The inspiratory effects of stimulation of the central end of the vagus so commonly described were difficult to reconcile with the prolonged expiratory pauses noted by others. For these very same reasons the past and present studies of vagal physiology still have much in common.

Rosenthals, ,Athembewegung und ihre Beziehung zum Nervus Vagus" (33), published in 1862 is the first thorough and systematic attempt to explain the control of breathing. Rosenthal thought that he had learned why so 
many workers had arrived at divergent findings for when he stimulated the superior laryngeal nerve he too was able to obtain the expiratory effects described for stimulation of the vagus nerve. He, therefore, concluded that when electrical excitation is strictly localized to the pulmonary vagal fibers inspiration is invariably produced. Only when current escapes to the superior laryngeal nerve, is expiration or inhibition of inspiration produced.

Rosenthal had demonstrated that extensive sensory denervation caused little change in breathing and, therefore, concluded that the respiratory center possessed the power of rhythmical automatic discharge when it was exposed to its natural stimulus of oxygen lack. He pictured a continous resistance to this discharge under the control of the pulmonary vagal fibers. A lowering

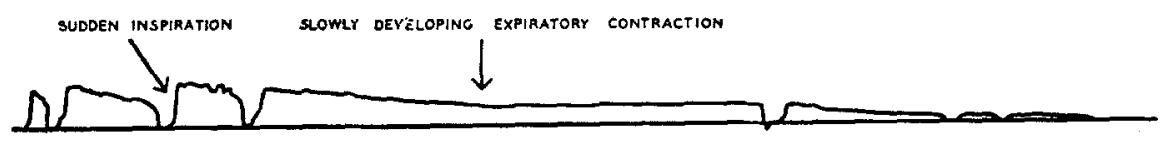

Fig, 48. Tracheal pressure record showing increasing expiratory activity as a result of increasing inspiratory filling of the lungs. The tracing reads from right to left. It was obtained with the use of a respiratory valve allowing movement of air into the lungs only. This old record of HeRING and Brever (Sitzgsber. ksl. Akad. Wiss. 58 II, 909 (1868)] demonstrating a progressively increasing intensity of expiratory contraction may be compared with adrantage to the electrogram of an expiratory muscle during a prolonged expiration produced by artificial inflation (fig. 68).

of this resistance by an increase in vagal activity was thought to increase the frequency of breathing, at the same time decreasing the depth of inspiration in direct proportion to the increase of frequency. The vagus nerves had no part in determining the total pulmonary ventilation. That was purely a central function depending upon the degree of oxygen lack. Like a tipping bucket, the center may be set for different ways of spilling. Thus RosEnTHAI believed that electrical stimulation of the vagus nerve decreases the resistance to the central discharges producing frequent but smaller discharges. Vagotomy on the other hand increases the resistance and produces less frequent but larger discharges.

Only six years later Hering and Breuer (80) proposed their famous theory of "Die Selbsteuerung der Atmung durch den Nervus Vagus" based largely on three simple findings: (1) inhibition of inspiration and excitation of expiration by artificial inflation of the lungs, (2) excitation of inspiration and inhibition of expiration on artificial deflation, and (3) slowing and deepening of respiration after vagotomy. According to their theory the normal inflation of the lungs during ordinary eupnea inhibits and cuts short the act of inspiration and excites the following expiration. Normal deflation of the lungs during the phase of expiration inhibits and cuts short the expiratory act and ushers in the following inspiration. This simple and direct theory seemed to reconcile the inspiratory and expiratory effects of central electrical stimulation of the vagus nerves described by so many. An original record by HERING and BRECER showing the expiratory action of pulmonary inflation 
is shown in fig. 48. The curve, which reads from right to left, is one of intratracheal pressure of a rabbit in which inspiration alone was allowed. As the lungs inflate with each inspiration, breathing is slowed by the "inhibition" of inspiration and the intratracheal pressure rises gradually long after each inflation, showing that active expiratory contraction is taking place (see table 1).

Table 1. Theories of pulmonary vagal function.

\begin{tabular}{|c|c|c|c|c|}
\hline & $\begin{array}{l}\text { HERING and } \\
\text { BREUER } \\
1868\end{array}$ & $\begin{array}{l}\text { GAD } \\
1880\end{array}$ & $\begin{array}{l}\text { HRAD } \\
1889\end{array}$ & $\begin{array}{l}\text { ADRIAN } \\
1933\end{array}$ \\
\hline \multirow[t]{2}{*}{$\begin{array}{l}\text { Inspiratory } \\
\text { Inflation }\end{array}$} & $\begin{array}{l}\text { 1. Inhibition } \\
\text { of Inspiration }\end{array}$ & $\begin{array}{l}\text { Inhibition of } \\
\text { Inspiration } \\
\text { Sole } \\
\text { Mechanism }\end{array}$ & $\begin{array}{l}\text { Inhibition of } \\
\text { Inspiration } \\
\text { Primary } \\
\text { Mechanism }\end{array}$ & $\begin{array}{c}\text { Inhibition of } \\
\text { Inspiration } \\
\text { Primary } \\
\text { Mechanism }\end{array}$ \\
\hline & $\begin{array}{l}\text { 2. Excitation } \\
\text { of Expiration }\end{array}$ & 0 & 0 & 0 \\
\hline \multirow{2}{*}{$\begin{array}{c}\text { Expiratory } \\
\text { Deflation }\end{array}$} & $\begin{array}{l}\text { 3. Inhibition } \\
\text { of Expiration }\end{array}$ & 0 & 0 & 0 \\
\hline & $\begin{array}{l}\text { 4. Excitation } \\
\text { of Inspiration }\end{array}$ & 0 & $\begin{array}{c}\text { Minor } \\
\text { Importance }\end{array}$ & $\begin{array}{c}\text { Minor } \\
\text { Importance }\end{array}$ \\
\hline
\end{tabular}

A summary of the outstanding studies of the reflex vagal control of breathing. Both pulmonary inflation and deflation are recognized as sources of reflex control by all of these studies with the exception of those of GAD. Hering and BreuEr gave importance to both controls but contrary to general opinion they did not insist upon the existence of two separate corresponding sets of vagal fibers. GAD ignored the reflexes arising from expiratory deflation entirely. While HeAD and ADRian recognized the existence of such reflexes they assigned a relatively small role to them, a role confined perhaps to abnormally small lung volumes. The identification of two sets of endings with two sets of respiratory reflexes has not been attained but LARSELLs histological description of pulmonary endings to which functions have not yet been assigned is not opposed to such possibilities. Our findings assign a predominant role to the afferent vagal impulses coming from the so called stretch receptors. These impulses impinging upon both inspiratory and expiratory half centers exert an alternating stimulating action on the individual centers as determined by the phase of activity which is prevailing. This view, however, does not exclude the possibility of other vagal reflexes. The fact that excessive deflation of the lungs produces a greater respiratory response than double vagal block, is suggestive of the existence of an independent collapse reflex (Moyer and Geselt, Unpublished).

GAD (81) in 1880 had demonstrated to his own satisfaction that pulmonary inflation actually elicited expiratory contraction and thus offered data supporting the views of Hering and Breuer. But he refused to place interpretation on his results which Hering and Breuer did on theirs. He could not convince himself that the expiratory contractions were strong enough to collapse the lungs sufficiently to set up an expiratory inhibitory and inspiratory excitatory reflex. He dropped the role of expiration as a factor of control and looked upon breathing as a purely inspiratory phenomenon. Like Rosenthal, he assumed central automaticity of discharge 
and a single modifying action of the vagus nerves. This action is inhibitory, developing during each natural inflation of the lungs and automatically shortens each inspiratory discharge. The inhibitory action of the vagus is thought to continue with decreasing intensity throughout the expiratory phase. Only as the "after-inhibition" wears off does central chemical stimulation gain the upper hand once more and start a new inspiratory discharge. This is in turn checked by the next filling of the lungs. These experiments of GAD mark the beginning of a growing emphasis of the inspiratory inhibitory action of the vagal stretch reflex. Why GADs experiments should have been interpreted as they were is hard to see for he showed not only that

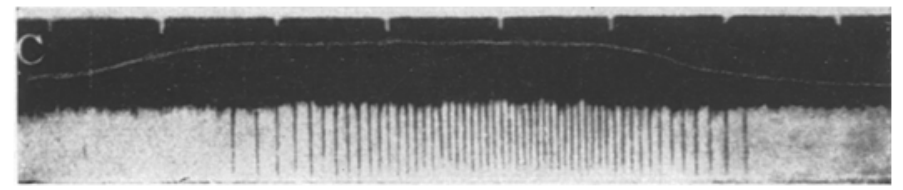

Fig. 49. Normal respiratory discharge of a single fiber of the vagus of a decerebrate cat. White line signals respiration and moves uprrards when lungs expand (tamboux connected to aix reservoir). Time marker gives $1 / 4$ sec. intervals. [ADRIAN: J. of Physiol. 79, 332 (1933).]

the inspiratory act proceeds at a higher velocity when the vagi are intact but that inflation of the lungs called forth expiratory activity as well. These surely are inspiratory excitatory and expiratory excitatory phenomena. But what was his proof of inspiratory inhibition?!!

In 1889 HEAD (82) published his experiments "On the Regulation of Respiration". This was the second classical paper on this subject to appear from Herings laboratory. He found that pulmonary inflation produces a prolonged pause in which inspiratory activity stops and that deflation produced a pause in which inspiratory activity was strong. Ultimately rhythm is established in both conditions indicating that automaticity is a central phenomenon. Head, like $G_{A D}$, abandons the role of the expiratory half center in the control of breathing and regards breathing as a purely inspiratory phenomenon. Hering and Breuer, it will be recalled, state that an increase of intrapulmonary pressure provokes expiratory activity. Head states that the inspiratory activity ceases when the lungs are inflated. Here lies the real issue in the problem of vagal function.

HEAD ends his theoretical discussion of the regulation of respiration as follows.

"In conchusion, the vagi produced two effects apon the centre during normal breathing. Firstly, each inspiratory contraction is checked when it has reached certain height owing to the stimulus caused by the dilatation of the lungs, and secondly, the inspiratory vitality of the centre is increased owing to these frequently repeated inhibitory stimuli."

The next very important study of vagal function was that of ADriax (83) on the "Afferent Impulses of the Vagus and their Effects upon Respiration" for he showed the manner in which the stretch receptors fire with changing 
volume of the lungs. A sample record of the gradually increasing discharge of a single receptor during normal inspiration of the rabbit is illustrated in fig. 49. With the more sudden emptying of the lungs during expiration the frequency falls some what more rapidly to zero. The activity of the receptors varies in different preparations. Some may show continuous discharges upon which the respiratory fluctuations are superimposed while others stop firing during the expiratory pause. The curves of frequency of these two types are plotted in fig. $50 \mathrm{~A}$ and $\mathrm{B}$.

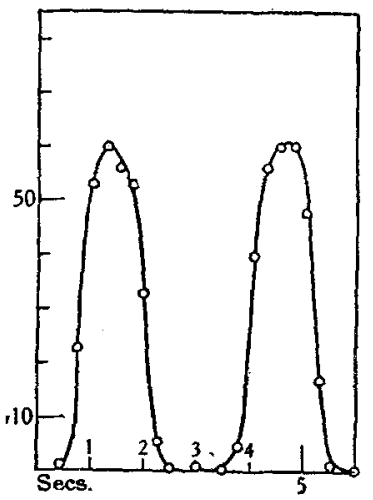

A

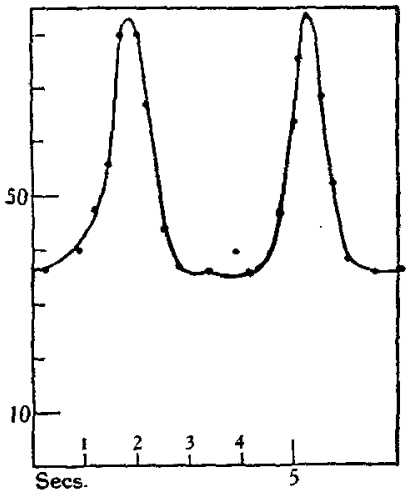

B

Fig. 50. Freouency of impulses in single fibres of the cat's vagus during normal breathing. Two preparations bath decerebrate. In preparation A the lungs collapse sufficiently to abolish all residual activity of the stretch receptor during the so-called "expiratory pause". In preparation B the stretch receptor remains distorted for it continues to discharge throughout the entire expiratory phase. [ADRIAN: J. of Physiol. 79, 332 (1933).]

By direct experiment, Adrran finds that artificial inflation of the lungs timed to come at the beginning of a natural inspiration checks that inspiratory

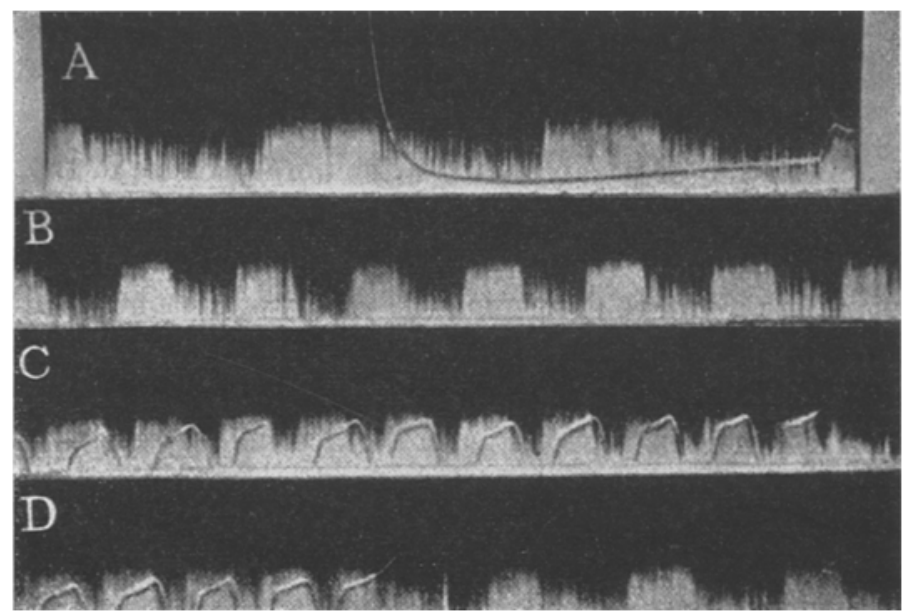

Fig. 51. Discharge of motor impulses in the top root of the phrenic nerve cut distally. Decerebrate cat. All records at same speed. A. both vagi blocked with novocaine. Slow rhythm (27 permin.) unaffected by sudden inflation of the lungs. Downward movement of signal marks inflation. B. Vagi intact. Normal breathing 58 per min. C. Vagi intact. Periodic inflation of lungs timed so as to inhibit each motor discharge. Downstroke of signal shows inflation. The rate increases to 90 per min. D. After 11 sec. the periodic inflation is stopped. The rate falls at once to 48 per min. Time marker (top record) gives $1 /{ }_{4}$ sec. intervals. (Adrias: J, of Physiol. 79, 332 (1933).)

act and steps up the frequency of breathing (see fig. 51). He interprets the vagus in much the same way as did HEAD for he believes the "dominant activity of the vagus is due to the stretch receptors". 
"They are inhibitory, but they can influence rhythm in either direction because inhibition of the phase of activity will shorten this part of the cycle and tend to hasten its return, but inhibition during the phase of rebuilding will delay it." "The maximum inhibitory effect occurs during the phase of activity and, therefore, the rhythm is usually quicker with intact than with cut vagi. The motor discharge will be curtailed, there will have been a smaller expenditure of active material and it is reasonable to suppose that less time will be taken in preparing for a fresh outburst. Thus a series of brief inhibitions at each expansion of the lungs should give a cycle consisting of brief motor discharges repeated at short intervals; whereas the absence of all inhibition should give longer discharges spaced at greater intervals."

"The opposite effect will be produced if the inhibitory message acts throughout the period when the center is recharging, for this will tend to delay the appearance of a fresh discharge.

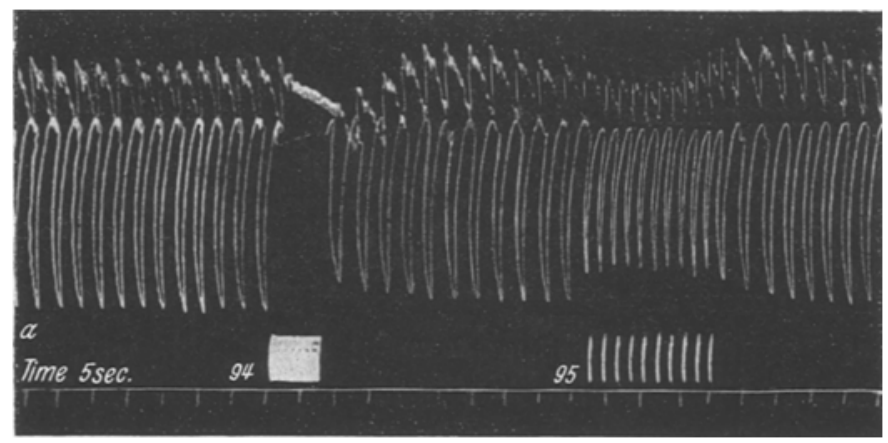

Fig. 52. Effects of continuous faradic and periodic faradic stimulation of the central end of the vagus nerve. Continuous stimulation produces "apnea" in the expiratory position. Stimulation timed to occur only during the inspiratory phase accelerates the frequency of breathing. [HILLENBRAND and BoYD: Amer. J. Physiol. 116, 380 (1936).]

Thus the rhythm, in so far as it is determined by the stretch receptors, will be most rapid when the inhibitory effect rises sharply at each inspiration and falls to zero at expiration."

See fig. $50 \mathrm{~A}$ and $\mathrm{B}$.

Like Rosenthal, Gad and Head, Adrian looks upon normal breathing as a purely inspiratory process. The phenomenon of expiration is barely mentioned. He states that "normally the expiratory activity occurs as an after effect of the inspiratory" and that. "An explanation on these lines was in fact given in the classical paper published by Henry Head from Herings laboratory in 1889 though it appeared then that the expiratory endings might play some part in the effect." The equally important fact that inspiration follows on expiration was not considered.

A significant point of agreement in the theories of Hering and Breuer, GAD, Head and ADRIAN is the emphasis of the role of inhibition of inspiration in the control of breathing. This view is more recently supported by the experiments of Hillenbrand and Boyd (84) and Boyd and MaAske (85). By automatically delivering tetanic stimulation to the central end of the cut vagi during the phase of inspiration, they were able to cut each inspiration short (see fig. 52) and thereby accelerate the frequency of breathing. The results are similar to the acceleration of breathing produced by artificial inspiratory 
inflation of the lungs described by Adrian. Continuous stimulation, stopped breathing in the expiratory position.

According to the views of many workers in the field of vagal physiology a single set of vagal receptors is considered adequate for a logical explanation of the control of breathing. AdRIAN particularly is of this opinion. HamMouda and Wrusor $(86,87,88)$, however, are of the opposite mind for they have been able to change the usual inhibition of breathing produced by faradic stimulation of the central end of the vagus nerve into one of accelerated breathing by a graded cold block placed central to the point of stimulation. At
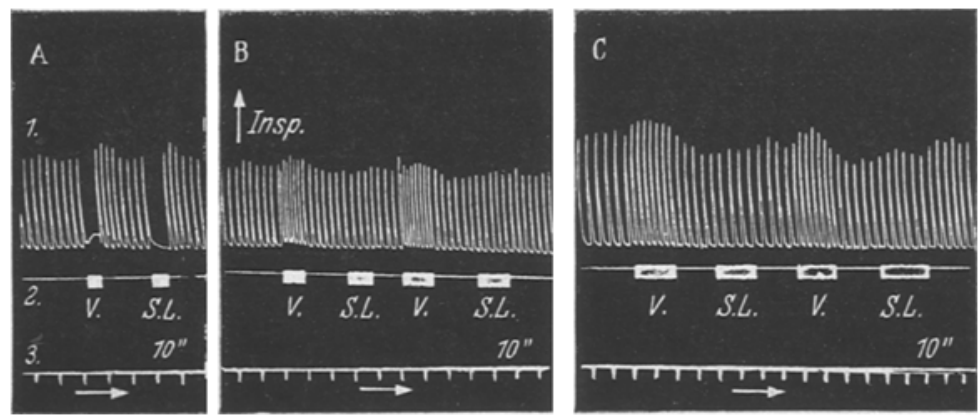

Fig. 53. Excitation of superior laryngeal and vagus nerves peripherally to a cooled area. Dog 4,6 kg. Medinal $1 \mathrm{~g}$. 1. stethograph record; 2. signal (faradization); 3 . time $10 \mathrm{sec} . \nabla$, stimulation of central end of cut right cervieal vagus. S. L., stimulation of central end of right superior laryngeal. A, both nerves on thermode at $10^{\circ} \mathrm{C}$. B, thermode at $3^{\circ} \mathrm{C}$. after section of left vagus (both vagi now cut). Stimulation by faradizing with coil at $5 \mathrm{~cm}$. peripheral to the cooled region of the nerves. [HAMmovos and WILson: J. of Physiol. 85, 62 (1935).]

blocking temperatures of approximately $5^{0} \mathrm{C}$ the inhibitory effect disappears and is substituted by one of acceleration (see fig. 53 and its legend). They conclude there exist "two functionally opposite types of vagus nerve endings in the lungs, the one augmentor, the other inhibitory of respiration, the former being excited by any changes in the volume of the lungs, the latter only by the increased tension of the lung tissues produced by expansion". The histological description of several types of pulmonary endings by LARSELL (89) is not averse to the views of HAMmouda and Wilson yet their findings are capable of another interpretation. We shall return to this point at a more opportune moment.

Of special interest are the findings of Hess (90,91 and 92) and his group. They arrive at a new interpretation of vagal function through their studies of diaphragmatic tone during pneumothorax. Super-inflation of the lungs not only produces the well-known slow and deep respiratory movements but in addition an elevation of the diaphragm, indicative of a loss of diaphragmatic tone. Release of air from the distended lungs increases the frequency and decreases the depth of breathing and increases the tonic activity of the diaphragm. Diaphragmatic tone varies inversely with the volume of the lungs or its equivalent, the intensity of vagal ending activity. This phenomenon 
is as demonstrable in the apneic as it is in the eupneic animal (see fig. 54). Thus Hess refuses to accept the theory of vagal interruption of breathing such
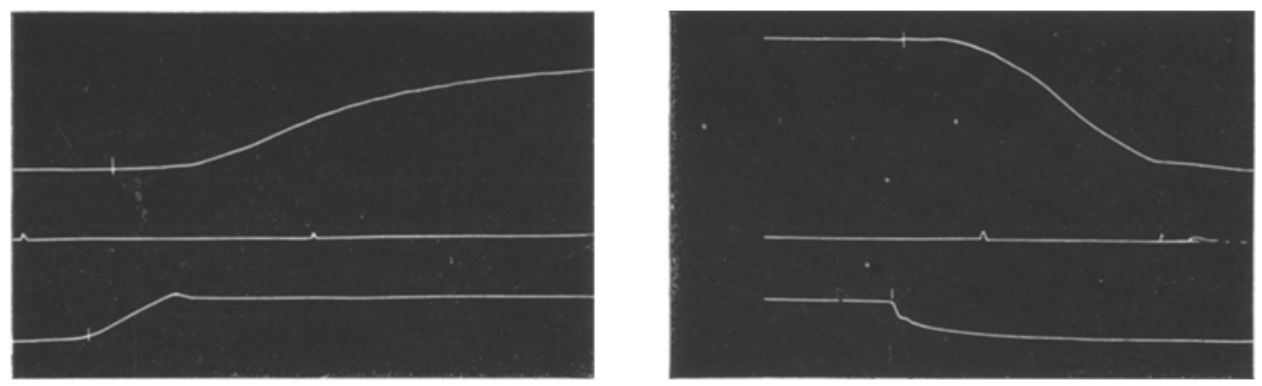

Fig. 54. Tonus changes of the diaphragm resulting from lung volume changes during hypocapnic apnea. Upon inflation of the lungs (lower curve in fig. 54a) the diaphragm relaxes ater a latent poriod of 0,22 second. Upon withdrawing air from the lungs (lower eurve in fig. $54 \mathrm{~b}$ ) the diaphragm contracts to its former position. Three seconds intervene between figures $a$ and $\mathrm{b}$. [IIEgs: Pflizgers Arch. 296, 198 (1930), ]

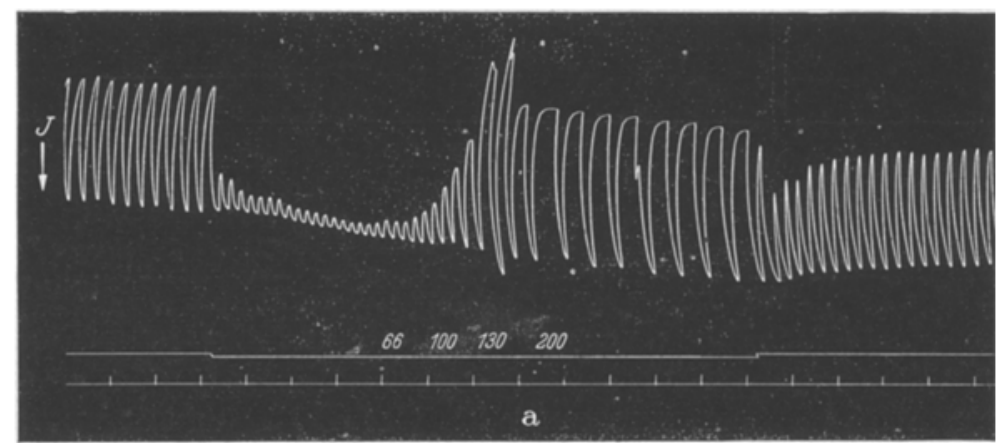

Fig. 55.

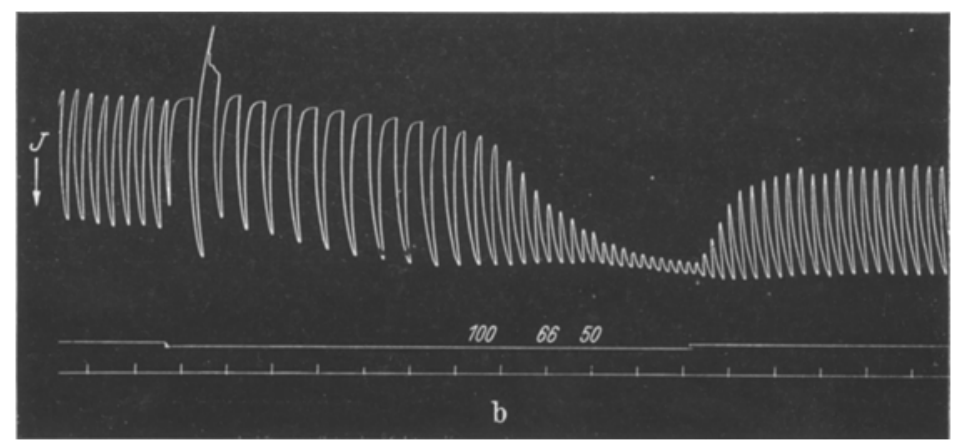

Fig. ว6.

Fig. 55 and 50. Lffects of afferent stimulation of the vagus gerve on respiration. A Increasing freuuency of stimulation from 66 to 200 per second. B Decreasing frequency. [WYss: Pflügers Arch. 242, 215 (1939).]

as originally proposed by Hering and Brever. He is rather of the opinion that the vagi determine the "Tonuslage" of the diaphragm and thus the frequency and depth of breathing. 
The limited freedom of movement of a highly tonic diaphragm and the corresponding small amplitude of contraction determines a short period of contraction and, therefore, a high frequency of breathing. The unlimited freedom of movement of a fully relaxed diaphragm on the other hand allows powerful and more infrequent contractions.

„Damit ist die Hauptwirkung des Vagus in der Regulierung von Atemtiefe und Atemfrequenz klargelegt, und zwar im Sinne einer Tonisierung und nicht einer Schaltfunktion!"

More recently [Wyss (193)] the effects of the vagal stretch reflex have been correlated with the normal frequency of discharge of the stretch receptors of the lungs as demonstrated by ADrian. As is known from this work the frequency of vagal discharge established in the rabbit varies from approximately 50 or less per second with the lungs in their normal expiratory position to about 350 in the highly inflated condition. Duplicating such activity by electrical stimulation of the vagus nerve Wyss (93) finds, as shown in fig. 55 , that a low frequency of stimulation of approximately 50 per second produces a highly tonic contraction of the diaphragm interrupted by frequent clonic contractions. A progressive increase in frequency of stimulation produces a progressively increasing relaxation of the diaphragm during the expiratory pause and an increasing amplitude of contraction. Wyss concludes that „Die Reizeffekte stimmen sehr weitgehend mit den Hessschen Lungendehnungsreflexen überein". RICE (174) also finds that frequency of stimulation determines the nature of the response (see

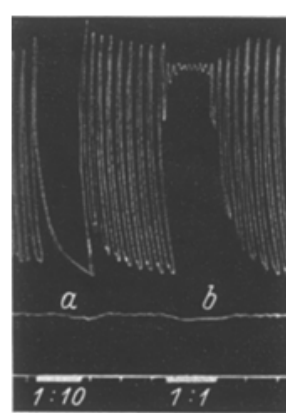

Fig. 57. High frequency stimulation of the cen. tral end of the vagus at left slows breathing in the expiratory position while low frequency stimulation accelerates it with the lungs in the inspiratory position.

[RICE: Amer.J. Physiol. 124, 535 (1938).] fig. 57). High frequency slowed breathing determines in the expiratory position and low frequency accelerated it in the inspiratory position.

Our views on vagal function are very different from any so far proposed for we look upon the vagal stretch reflex as a purely excitatory phenomenon [Geselu (38, 39, 40, 41 and 94)]. As already pictured for the chemoceptors and nociceptors we see the impulses initiated in the stretch receptors of the lungs distributed to both inspiratory and expiratory half centers (see fig. 58). During inspiration, while the lungs are expanding, the inspiratory center is stimulated with an increasing intensity proportional to the increasing discharges of the vagal proprioceptive endings. During the expiratory phase of breathing the vagal drive is shifted to the expiratory half center which is now stimulated with decreasing intensity proportional to the waning activity of the vagal endings.

The experimental proof of this theory of vagal action is abundant. The most direct comes from the more recent studies of the action potentials of inspiratory and expiratory muscles [Worzniak and Geselu (97)]. For example 
the weak eupneic contraction of the diaphragm, represented in the action potentials of the phrenic nerve in fig. $59 \mathrm{~A}$ is markedly increased in strength, by a superinflation of the lungs during the following inspiratory act. This augmentation of the inspiratory contraction is no doubt due to a strengthening of the normal vagal stretch reflex for if the vagus nerves are blocked the effects are missing. Fig. 59 B shows that augmented frequency of twitch and

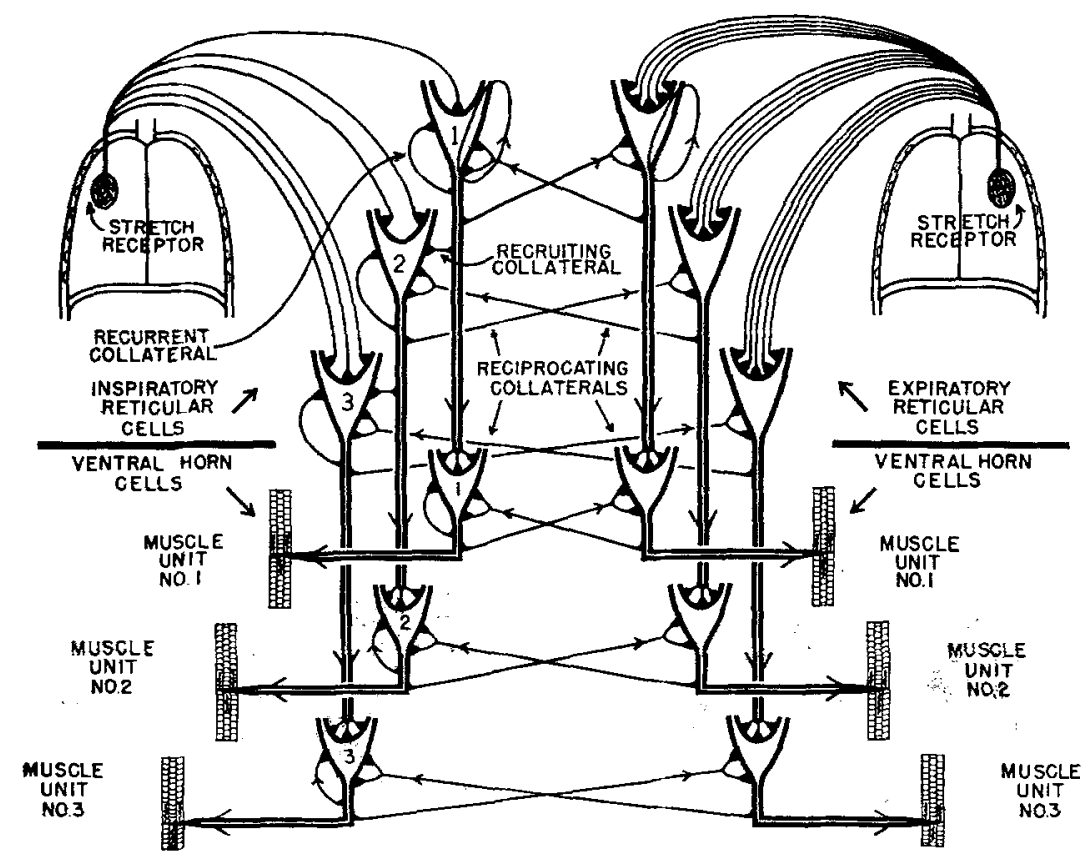

Fig. 58. Schematic representation of the central connections of the vagal stretch receptors and their quantitative distribution. Because the vagus is a powerful expiratory nerve copious connections are allotted to the expiratory side. Because the normal stretch reflex which occurs during each inspiration is capable of a quickening without a deepening of inspiration the vagal fibers are arranged in a way designed to facilitate a rapid development of the inspiratory discharge. Note that reticular cell \#1 receives only one vagal fiber, that cell \# 2 receives 2 fibers, ete. That would tend to bring the electrotonic excitation currents of all cells close to that of pace setter \# 1 as the lungs stretch and allow the inspiratory wave to pass through the center at a greater speed. [GesekL; Unir. Hosp. Bull. (Michigan) 5,12 (1939).]

recruitment of new active units participate in the strengthening of contraction. During eupnea, muscle unit \# 1 twitched but six times and at a relatively low frequency. In the second inspiration it twitched twice as often, at a higher frequency and for a longer period of time. One new active unit was momentarily recruited at the very end of the discharge.

In fig. 60 a similar strengthening of the expiratory contraction of the internal abdominal oblique muscle occurs when the lungs are inflated during the expiratory phase. A new steady state of activity is established in which the individual units twitch at a higher steady state frequency and in which new active units are added. The augmented frequencies of the individual units indicate a greater stimulation of the individual cells of the expiratory 


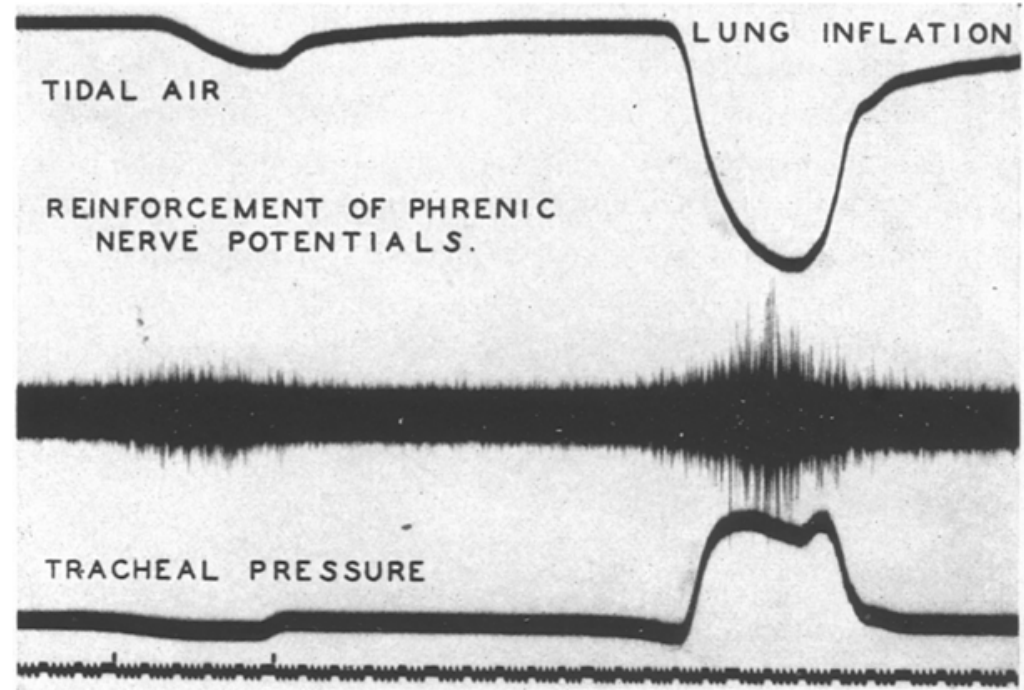

$A$

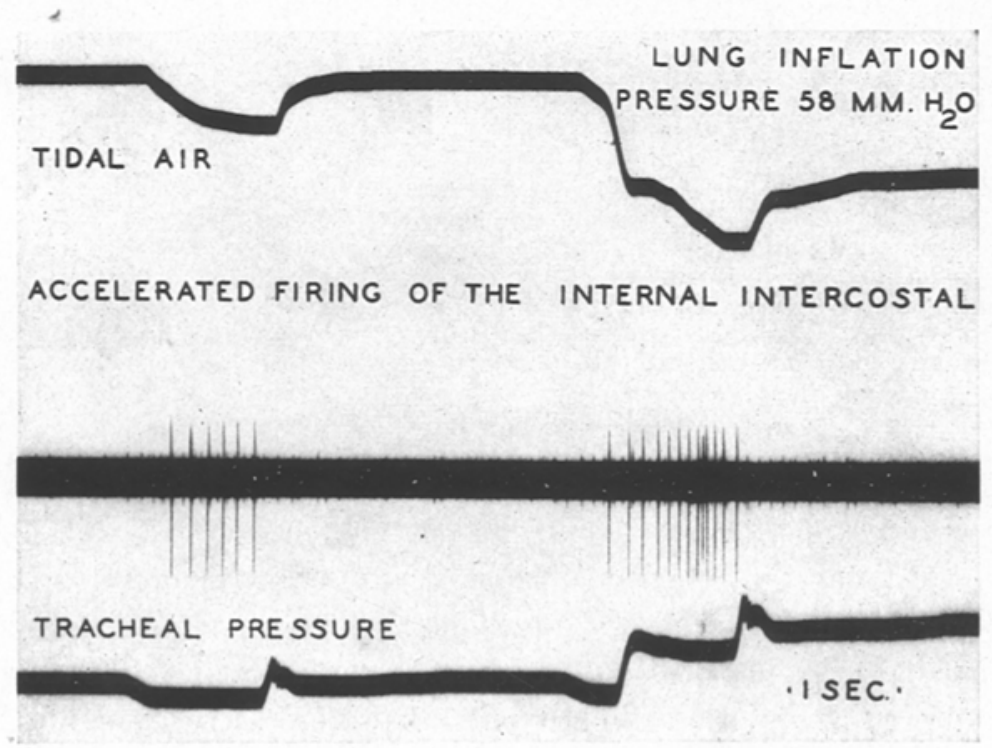

B

Fig. 59. Record A. Augmentation of the normal inspiratory fusillade of the phrenic nerve, at the left, to a powerful discharge, at the right, by artificial superinflation of the lungs. Inflation is produced by weighting the mercury spirometer (fig. 64) just as inspiration begins. The degree of inflation and the tracheal pressure change are indicated by the tidal air and tracheal pressure records.

Record B. A similar augmentation of inspiration by a superimposed inspiratory inflation of the lungs recorded directly from the internal intercostal muscle. Comparison of the normal and modified contraction shows that accelerated frequency of twitch and recruitment participate in the strengthening of the contraction. The newly recruited unit gives only one twitch, seen between twitch 8 and 9 of muscle unit 1 . [WorzNIAK and Gesilu: Amer. J. Physiol. 126, 658 (1939).]

half center and thus explain the recruiting of new active units out of the subliminal fringe. 
While such results as these are readily obtained, they are by no means uniform. Augmentation of expiratory activity, as a matter of fact, is often replaced by inhibition due, we believe, to interfering stretch reflexes set up in the proprioceptive endings of the muscles studied. At least, the results become more uniform when studies are made in the larynx where local stretching is easily avoided during pulmonary inflation. In these experiments rhythmic artificial ventilation proved to be a most valuable procedure for revealing the principles of proprioceptive drive. As is well-known, expiration is readily

\section{ACCELERATED FIRING AND RECRUITMENT IN THE INTERNAL ABDOMINAL OBLIQUE}

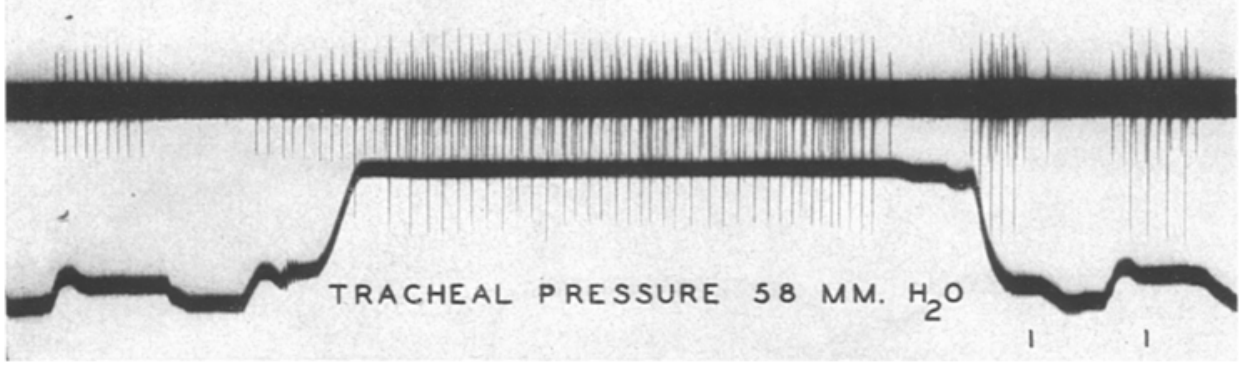

Fig. 60. Augmentation of expiratory activity by superinflation of the lungs, timed to come during the expiratory phase. The first discharge and the introductory twitches of the second discharge represent normal expiratory activity of the steady state type registered in the internal abdominal oblique muscle. Careful inspection of the electrogram will show an immediate acceleration of discharge of unit \#1 and the recruitment of an additional active unit \# 2 , when superinflation of the lungs occurs. The augmented expiratory activity is accompanied by a prolongation of the expiratory phase, which is interrupted by inspiration just preceding the end of the period of superinflation of the lungs. The expiratory discharges following unloading of the lungs are stronger than the preinflation discharges due to the asphyxial changes which develop during the prolonged expiratory activity. This disappears in a few breaths. [WoRzNIAK and GFSELL: Amer. J. Physiol, 126, 658 (1939).]

brought into phase with the pump. When that happens, as was the case in fig. 61, the thyroarytenoid muscle, which is expiratory in function, responded in a beautifully mechanized way with increasing frequency of twitch with each inflation of the lungs. Upon collapse of the lungs, which is more sudden than the preceding inflation, the discharge fell off with corresponding abruptness. Comparison with Fig. 49 will show how nicely the electrogram of the thyroarytenoid muscle follows the electrogram of the vagus sensory fiber in the rabbit. It is thus evident that an expiratory muscle can be made to contract in the slowly augmenting manner typical of an inspiratory musele if proper conditions are provided. So too, it can be made to contract in the rapidly augmenting manner under conditions of waning vagal drive normally obtaining during expixation. Artificial ventilation e.g. may establish respiratory rhythm in which inspiratory activity is in phase with inflation of the lungs and even if the pump is temporarily stopped breathing may continue momentarily in the same tempo while the lungs are completely collapsed 
(see fig. 62). In this state of lung deflation vagal drive is zero. We may then compare expiratory contraction occurring during zero vagal drive and

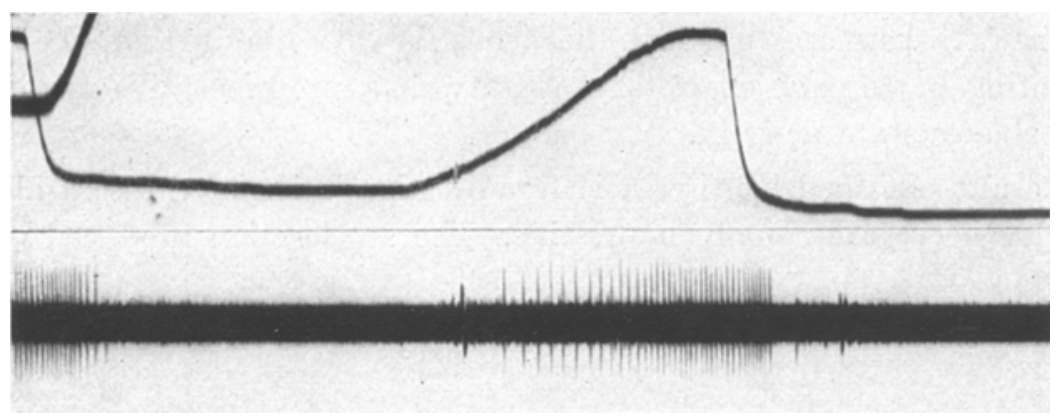

Fig. 61. Vagal reflexogenic drive of an expiratory muscle during periodic artificial inflation of the lungs. The slowly increasing inflation, indicated by the rising gradient of the upper record corresponds with the ejection stroke of the pump. Passive elastic emptying of the lungs through an automatically opened valve proceeds with greater speed. The frequency of twitch of the thyroarytenoid muscle corresponds with the expected degree of stretch of the pulmonary vagal endings and duplicates ADRIANs records of vagal fiber discharge (fig. 49). The slower falling off of vagal fiber discharge in ADRtans experiments is probably due to a slower emptying of the lungs. The record of thyroarytenoid activity was obtained with the animal in the apneic condition. In conjunction with the findings of Hess (fig. 54) it shows that both clonic and tonic activity of respiratory muscles, inspiratory and expiratory are elicitable through the vagal stretch reflex. [Worzniak and Gesell: Amer. J. Physiol. 126, 658 (1939).]

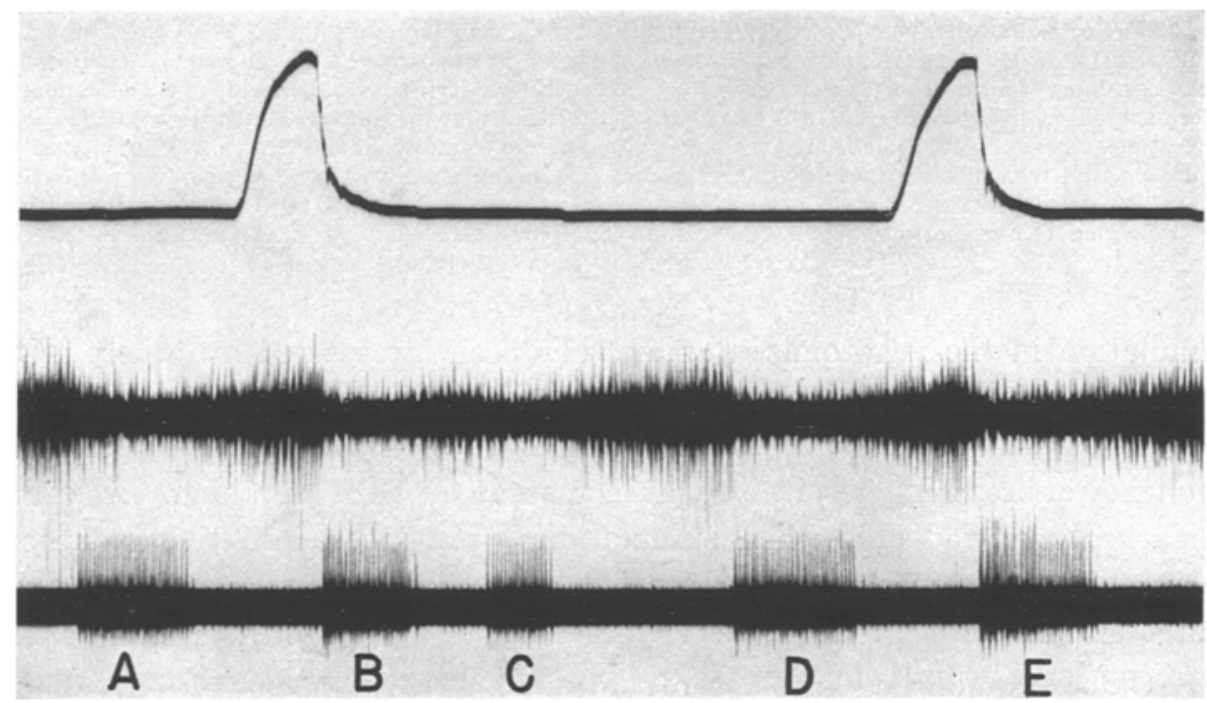

Fig. 62. Vagal reflexogenic drive of an expiratory musele during periodie arificial fentilation of the lungs with the animal in the non-apneic state. In this record inspiratory activity, upper electrogram, is in phase with the inflation stroke of the pump. Deflation of the lungs and therefore diminishing vagal drive occurs in normal time relation with the expiratory activity at $\mathrm{B}$ and $\mathrm{E}$. Between $\mathrm{B}$ and $\mathrm{E}$ there are two complete respiratory cycles during which the lungs are completely collapsed. Vagal expiratory drive for expiration $\mathrm{C}$ and $\mathrm{D}$ is, therefore, zero. The steady state expiratory activity at $\mathrm{C}$ and $\mathrm{D}$ and the rapidly augmenting activity at $B$ and $E$ conform with corresponding vagal activity, [WoRzNAK and GESELL: Amer. J. Physiol. 126, 658 (1939).]

during normal vagal drive of waning intensity. Inspection of the records show that expirations $\mathrm{B}$ and $\mathrm{E}$ occurring during waning vagal drive correspond 
with the theoretically expected rapidly augmenting type and that the intervening expirations $C$ and $D$ occurring during uniform zero vagal drive correspond with the theoretically expected steady state type of activity. These experiments, therefore, confirm the tentative hypothesis that the rapidly augmenting pattern of expiratory activity is a proprioceptive modification of the steady state activity.

Results obtained by rhythmic faradic stimulation of the central end of the vagus nerve are equally impressive. The acceleration produced by such

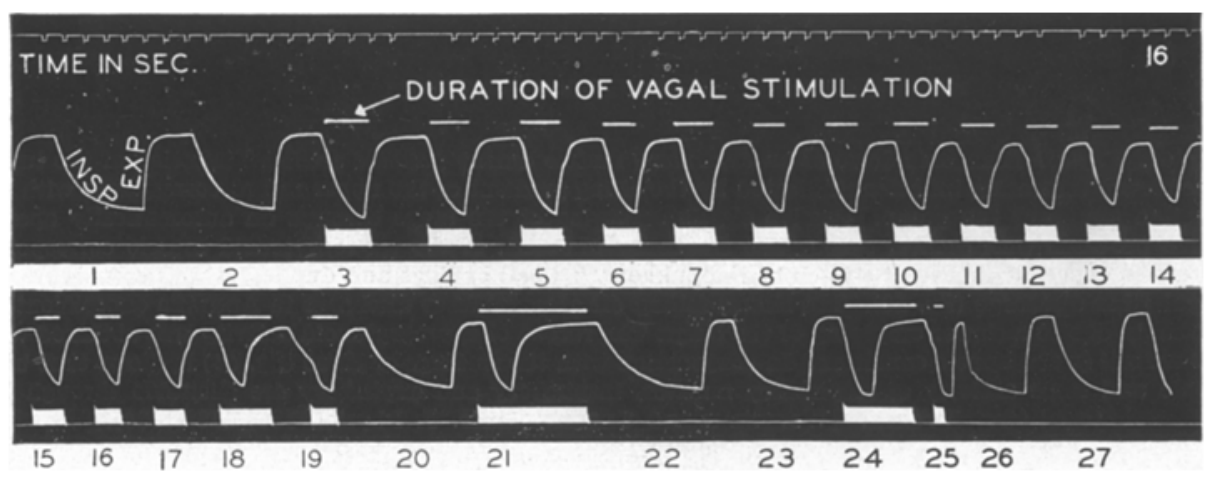

Fig. 63. Effects of afferent stimulation of the vagus nerve in the vagotomized dog. The two introductory respirations show slowly developing inspirations, apneustic in appearance. Faradic stimulation applied at the beginning of inspiration accelerates that act tremendously. Artificial interruption of each stimulation before the onset of expiration, as in 3 to 19 , accelerates the frequency of breathing, even in the earlier inspirations where the tidal air is slightly increased. Faradic stimulation accelerates the velocity of the inspiratory act regardless of the particular phase of inspiration during which it occurs (see respirations 19 and 25). Prolongation of stimulation into the expiratory phase retards the frequency of breathing by prolongiag the expiratory phase (see respiration 21). [GESELI, STEFFEnsen and BRookHakT: Amer. J. Physiol. 120, 105 (1938).]

stimulation in the vagotomized animal has been interpreted by others as an inhibitory phenomenon, but it will be seen that each stimulation increases the velocity of each inspiratory act to an extraordinary degree, and also the frequency of breathing despite a slight increase in depth of breathing. Breathing is markedly slowed only when stimulation is prolonged into the expiratory phase. This is due, as already suggested, to excitation of the expiratory half center and its associated reciprocal inhibition of the inspiratory half center.

Older unpublished findings of Moyer and Geselu now become more understandable (see fig. 65). In these observations on the dog the lungs are first inflated during eupneic breathing by weighting a specially constructed steel spirometer made to float on mercury (fig. 64). The vagi are then blocked and later deblocked, and after an interval of two minutes blocked and deblocked again. Finally the excessive pressure on the lungs is released. The records, from above downward, are costal, and abdominal respiratory excursions made with encircling bands, tidal air excursions made with the weighted spirometer, and mean blood pressure. Downstroke in the respiratory records 
indicates inspiration. The degree of inflation is visible in the tidal air record below.

Pulmonary inflation produces two immediate effects-cessation of breathing and a constriction of the abdomen. The first effect has, in the past, been regarded as a direct inhibitory action of the vagi on the inspiratory half center (Gad, Head and Adrian and others), but the contraction of the abdominal expiratory muscles and the extremely powerful respiration which bursts through the apnea, all overlooked by these workers, speak against such interpretation. Certainly the contraction of the expiratory muscles must be interpreted as an excitatory action (see also the prolonged expiratory contraction in fig. 68). Thus granting a reciprocating activity between the inspiratory and expiratory half centers the "inhibition" is readily understood.

The extremely rapid and powerful respiratory act interrupting the apnea is a most striking phenomenon. Note how sharply the spirometer record descends (fig. 65) and how forcibly the air is reexpelled without a moment's pause. This unusually abrupt intensification of inspiration and expiration cannot be explained by an increased chemical drive ensuing from the preceding apnea for these striking effects disappear in the following vagal block during which chemical stimulation is very similar. The abrupt stacatto breathing changes into a more smoothly swinging respiration, as noted by the lesser inspiratory and expiratory gradients and the greater spreading of the limbs. We

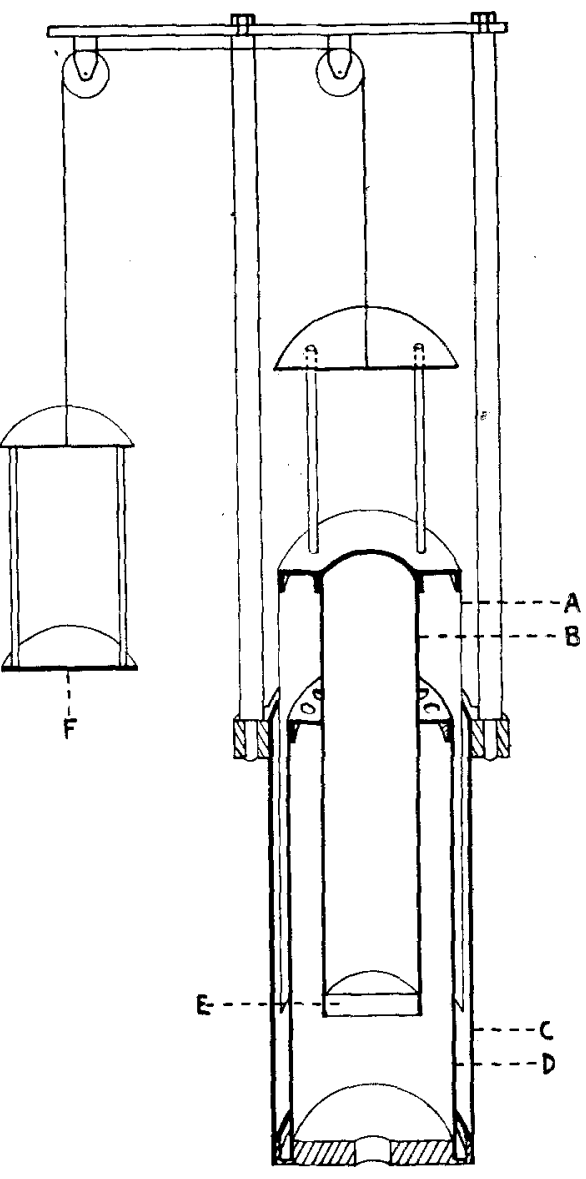

Fig. 64. Steel mercury spirometer used for studying effects of graded positive and negative intrapulmonary pressures. A Wall of steel spirometer. B Inner cylinder for receiving weights supported at $\mathrm{E}$. C and D Concentric steel tubes. The space between these tubes contains the mercury on which the spirometer is floated. F Pan for counterweighting to produce negative intrapulmonary pressures. [GESELL and Moyer: Quart. J. exper. Physiol. 25, 13 (1935).] are consequently forced to conclude that the augmented vagal discharge produced by superinflation of the lungs is the cause of the extreme speeding of the inspiratory and expiratory acts. Thus it becomes clear why the normal inspiratory act proceeds at a higher velocity as compared with that following vagal block when vagal drive is missing as was earlier noted by Gad (81), Nichocson and Brezin (120), Geselc, Steffensen 
and BrookнаRт (105). An interesting illustration on that point is seen in fig. 66, in which the velocities of inspiratory and expiratory contractions in a single
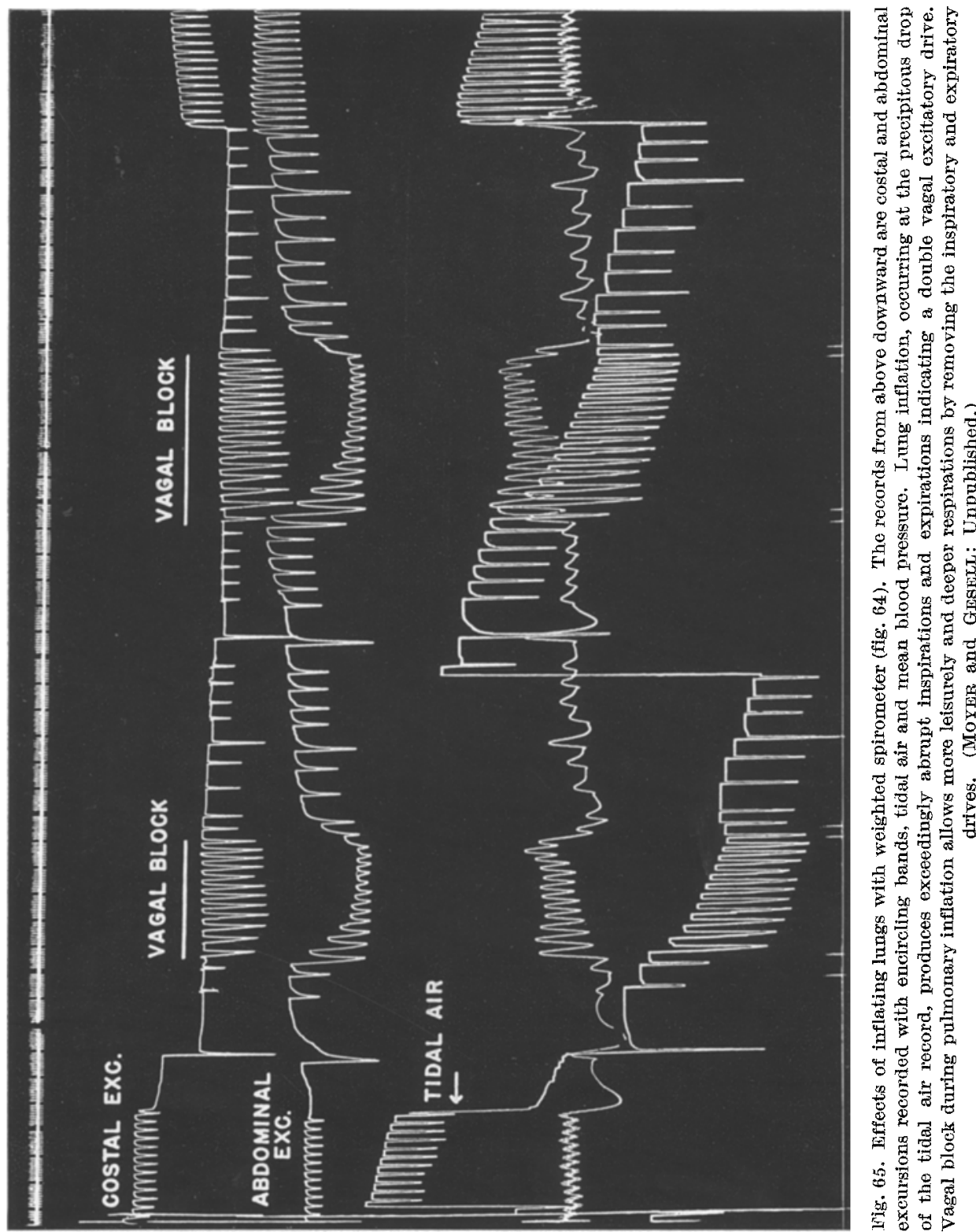

animal correspond with three intensities of vagal drive - 1. zero drive, during vagotomy, 2. normal drive, during eupnea and 3. supernormal drive, during superinflation. The inspiratory and expiratory gradients tell the changes which occurred. For completeness, it should however be added that frequently expiration is actually retarded by superinflation of the lungs. We attribute 
such results to other conflicting proprioceptive reflexes set up in the muscles themselves.

The mechanism by which vagal reflexes augment contractions would seem to be relatively simple. The neuro-mechanical circuit will illustrate
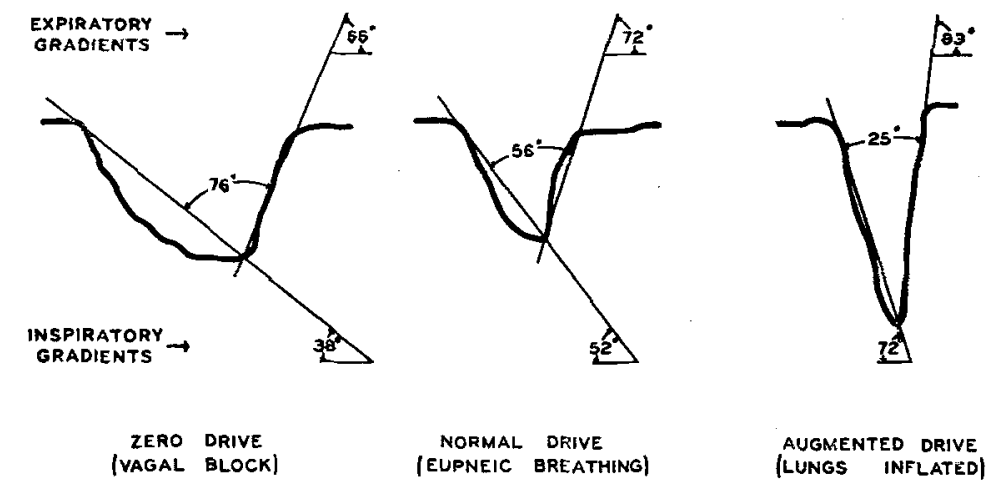

AUGMENTED DRIVE

Fig. 66. Velocities of inspiratory and expiratory contractions under three intensities of vagal drive. (MoyeR and GEsel.: Unpublished.)

(fig. 67). As a beginning the inspiratory reticular cells are pictured as firing in the slowly augmenting inherent pattern of discharge, demonstrated in the curare experiments. The inspiratory motor cells and muscles must necessarily follow suit. As the lungs distend, the degree of activation of the individual stretch receptors increases. The signals so set up travel on to the primary sensory nuclei and establish the slowy augmenting pattern of activity at these stations. This activity is in turn relayed back to the inspiratory reticular cells and thus superimposes upon the original reticular cell discharge. The complete result is a circulating self accumulative action which steepens the activity of the inspi-

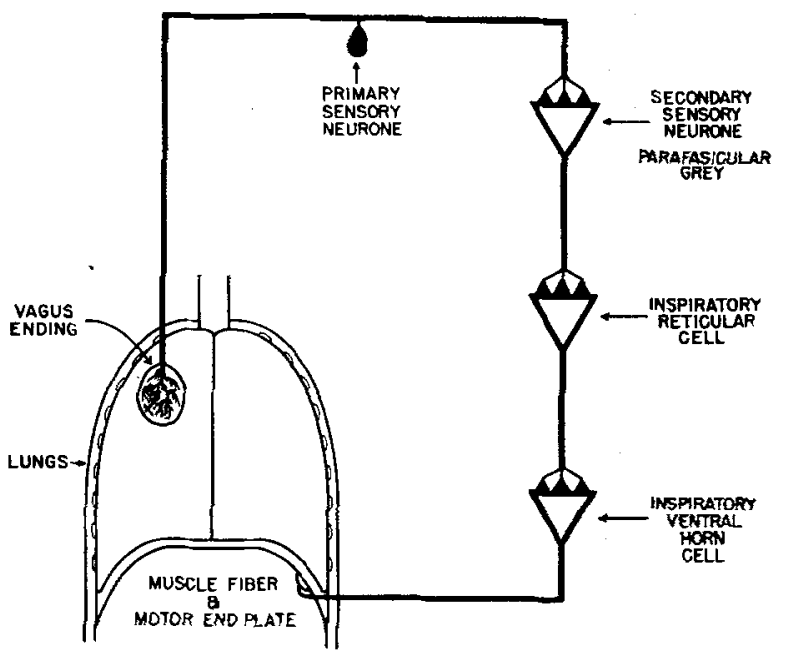

Fig. 67. A hypothetical neuromechanical circuit illustrating how the vagal reflex drive builds upon the slowly angmenting discharge of the inspiratory center. Inspiratory contraction stretches the vagal endings which fire back on the center to increase contraction still more and so on. (GEselt: Heart, Blood and Cirenlation, 1940.) ratory half-center (see fig. 174).

It may be asked- if the vagal proprioceptor signals are excitatory why do they not increase, instead of decrease, the depth of breathing? Oftentimes they actually do bring about a deeper respiration, witness, for example, the strong inspiration bursting out of vagal apnea in fig. 65. Though the vagi 
drive and intensify inspiratory contraction, they also check the act. Depth of breathing becomes a matter of balance between drive and check. Furthermore drive and check are most intimately related if our theory developed in section 14 is correct. As proposed there the recurrent collateral signals increase the frequency of discharge and by a process of exhaustion produce a disproportionate rise of the excitatory threshold as compared with that of the

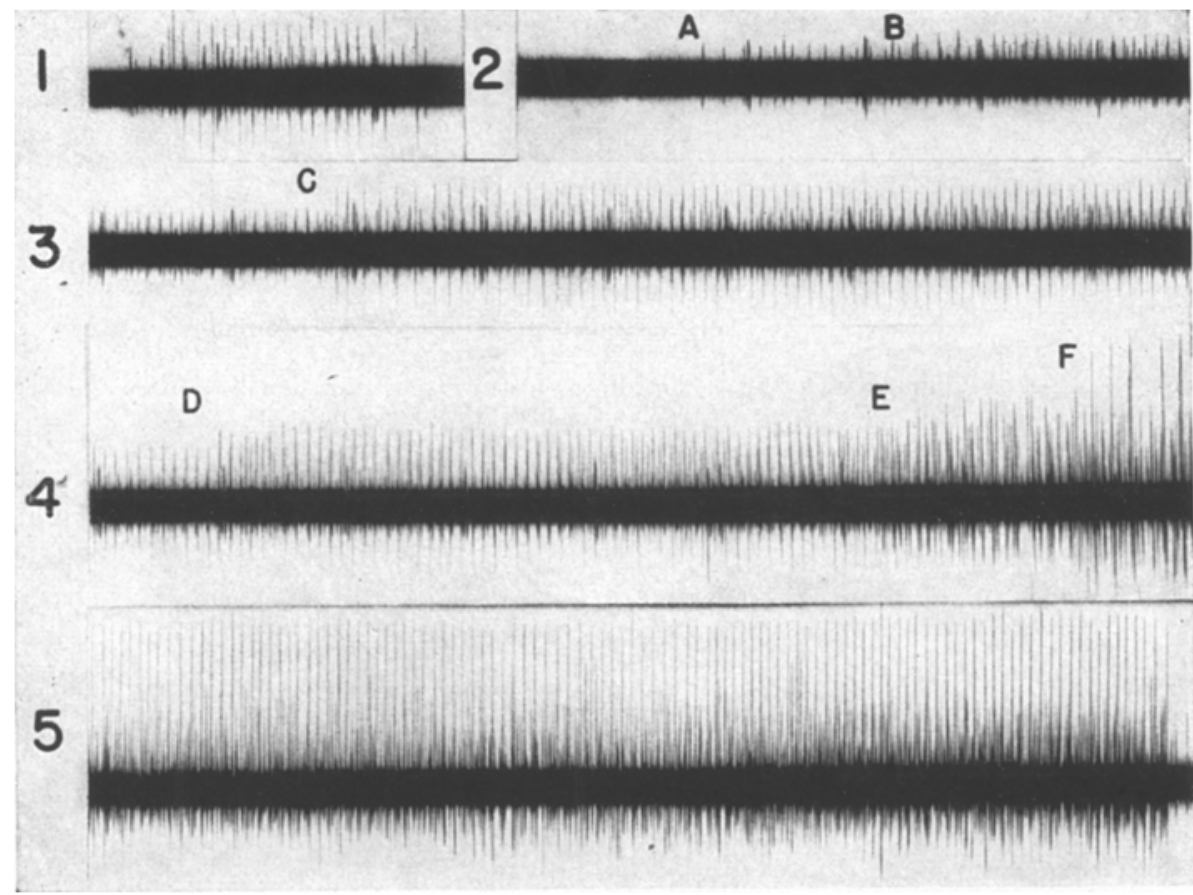

Fig. 68. Prolongation of expiratory activity by an intensification of the vagal stretch reflex (sustained inflation of the lungs) plus a progressive strengthening of the expiratory contraction by the additive action of the increasing asphyxial chemical drive. Record 1 is a normal expiration. Records 2, 3, 4 and 5 represent the dual proprioceptive and chemical modification of the normal expiration. The beginning of the modified expirations is weaker than the normal preceding expiration, differing in that respect from the results of fig. 60. This difference is probably attributable to a stronger opposing action of the inhibitory muscle spindle reflex in fig. 68 (see below) which however is soon overcome. A, B, C, D, E and F mark the recruitment of new active muscle units. Note the gradual increasing frequency of several units corresponding with increasing chemical drive. [WorzNaAK and Gesell: Amer. J. Physiol. 126, 659 (1939).]

accompanying rising electrotonic current. Thus working in conjunction with the recurrent collateral the vagus would assist in the interruption of the continuous discharge of the reticular cells. As exhaustion of these cells increases with each inspiratory discharge, the vagal drive upon the expiratory cells also increases, thus building a potential reciprocal inspiratory inhibitory action of increasing strength. The slightest weakening of the inspiratory discharge would release the expiratory cells from their state of inhibition whereupon they would instantly discharge. By virtue of reciprocal inhibition the inspiratory discharge would come suddenly to an end. Thus we look upon interruption of inspiration as a combined effect of exhaustion through direct 
reflex stimulation plus inhibition created indirectly through excitation of the expiratory half center.

\section{The Reflexogenic Support of the Grolar Signals.}

Litrue is known of the function of the Gougr endings and muscle spindles. As Creed, Denny-Brown, Ecches, Liddell and Sherrington (59) have said. "In the existing state of our knowledge it would evidently be rash to

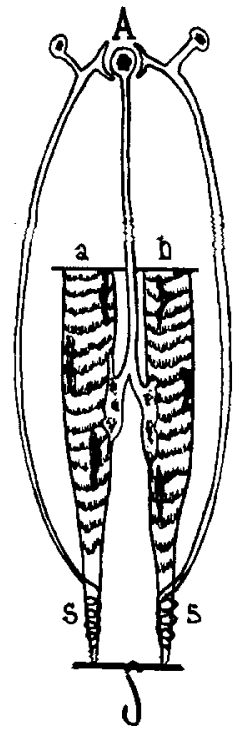

Fig. 69. A diagram showing the mechanical conditions obtaining when muscle end organs are disposed "in series" with the tension supporting elements. Under these circumstances tension, whether active or passive, will affect the endorgans indiscriminately, a and b, skeletal muscle fibres; $A$ anterior horn cell. [FULTON and PT-SuñER: Amer. J. Physiol. 83, 554 (1928).]

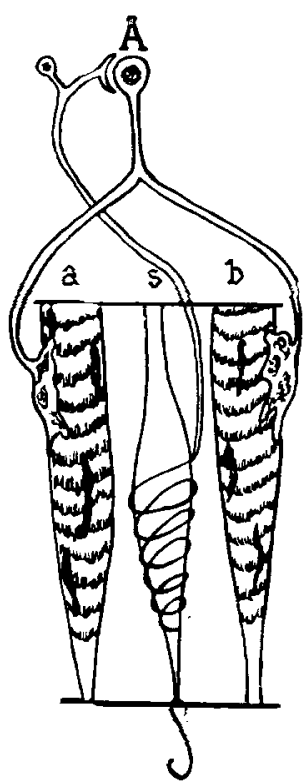

Fig. 70. A diagram to show the mechanical conditions obtaining when a muscle end-organ is arranged "in paraljel" with the tension supporting elements. $a$ and $b$, skeletal muscle fibres; s afferent end-organ associated with a fibre running parallel with $a$ and $b$ (e. g., an intrafusal fibre); A anterior horn cell. [FULTON and PI-SUÑER: Amer. J. Physiol. 83, 554 (1928).]

attempt to specify the reflex functions of any of the proprioceptive endings which have been deseribed." Nevertheless it seems desirable to propose \& temporary hypothesis in line with the reinforcing principles laid down for the ragal endings. Futton and Pr Suñer (96) have called attention to the parallel arrangement of the muscle spindles and the contractile muscle fibers and to the theoretical reduction of strain on these receptors accompanying muscular contraction (see fig. 70). Muscle spindle firing should accordingly diminish during contraction as demonstrated by Matthews (13). The firing of the GoLGr endings should on the other hand increase with increasing tension accompanying muscular contraction (see fig. 69). On that basis we have assumed that it is the stimalation of the Gougr endings which gives rise to the well-known stretch reflex. This reflex we believe is important in the 
adjustment of the mechanics of breathing. As normal inspiration progresses and meets with increasing resistance, the inspiratory Golgi signals increase in numbers and thereby provide the accessory reflex drive needed to overcome the increasing resistance. On that basis the mechanism becomes nothing more than a purposively graded stretch reflex such as already postulated for the vagal stretch reflex. Evidence supporting this view is found in the comparison of action potentials of inspiratory muscles-first during a normal inspiration when little resistance is met and second during occlusion of the

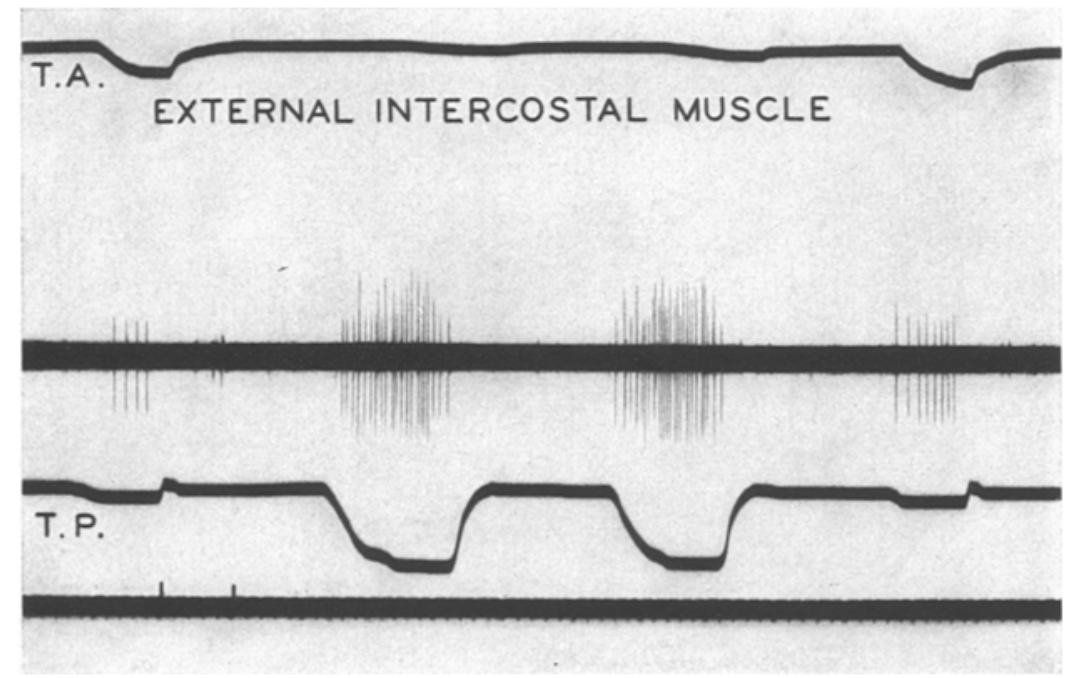

Fig. 71. Augmentation of GoLeI reflexogenic support of inspiratory contraction, illustrated in the electrogram of the external intercostal muscle. Occlusion of the trachea is designed to increase the tension and discharge of the GoLGI endings. Free breathing occurs in breaths 1 and 4; obstructed breathing in breaths 2 and 3. TA represents tidal air and T. P. trachea pressure. Inspection of the electrogram shows an earlier and more frequent twitch of muscle unit $\# 1$ and a recruitment of new active units in the obstructed inspirations. Recruitment is greater in breath 3 than in breath 4, another example of the addition of chemical to proprioceptive drive (compare with fig. 68). The greater post-occlusion as compared with the preocelusion discharge is an expression of the augmented chemical drive. [WORzNiAK and Gesele: Amer. J. Physiol. 126, 568 (1939).]

trachea when resistance is enormously increased (see fig. 71). The increased frequency of twitch and of recruitment during obstructed breathing is similar to that noted for the vagal stretch reflex and to that of increasing chemical stimulation. The increased inspiratory activity is undoubtedly due to the muscle stretch reflex for the effects are still obtainable after double vagotomy. It is, therefore, concluded that at least two proprioceptive reinforcing reflexes exist which are available for overcoming such resistance as may develop during the course of an inspiration, one coming through the vagal endings of the lungs and the other through the GoLar endings of the muscle. It is most significant that the phenomena of accelerated frequency of twitch and of recruitment of new active units evoked by a modification of proprioceptive activity are as readily demonstrable throughout the stations of the inspiratory 
arcs as are the basic patterns of activity in eupnea [MAGeE, Bricken and Geselu (97)]. Fig. 72 e.g. compares the phenomenon of accelaration of
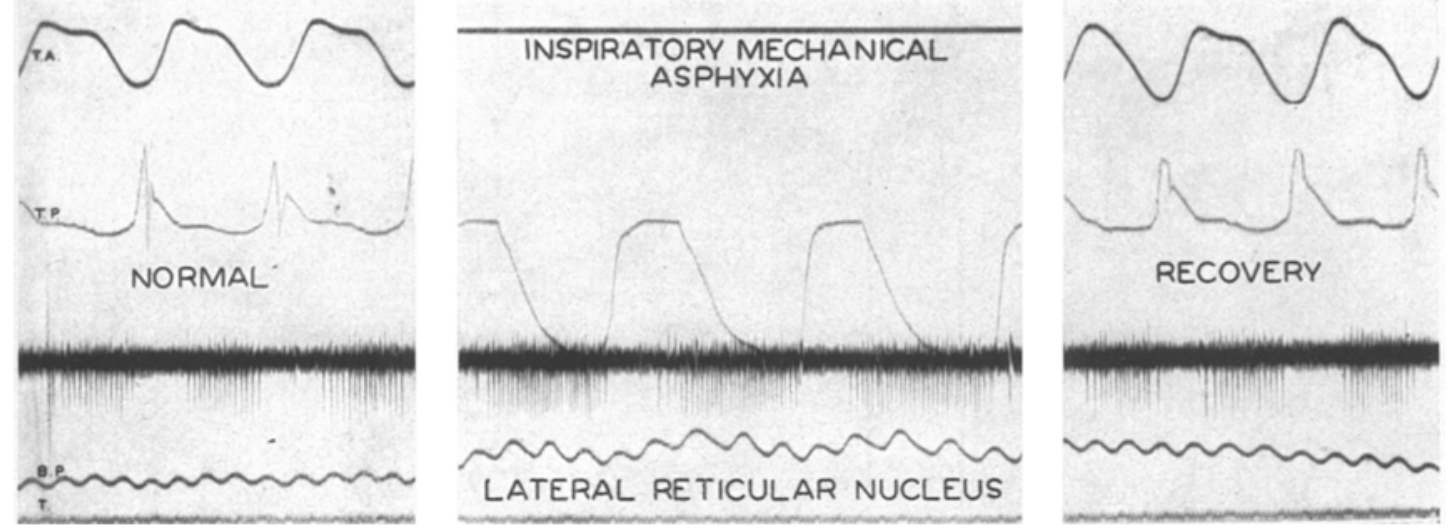

Fig. 72. Augmentation of GoLar reflexogenic support of inspiratory contraction as revealed by the acceleration and prolongation of inspiratory discharges in the lateral reticular nucleus. The middle record shows respiratory activity during tracheal occlusion. The end records show the normal and recovery controls. [MAget, Bricker and Gesele: Amer. J. Physiol. 129, 415 (1940).]

discharge in the lateral reticular nucleus during normal and obstructed inspiration. Fig. 73 shows both acceleration and recruitment in the internal arcuate fibers. The demonstration of increased activity in this sensory region is important in revealing that impulses giving rise to the respiratory stretch reflexes ascend the cord, to impinge on the secondary sensory nuclei (see fig. 74). From there the effects are most probably relayed to the reticular cells and thus on to the ventral horn cells.

The train of events occurring in the neuro-mechanical circuit during a normal inspiratory act are pictured as follows. The reticular and ventral horn cells begin to discharge in the slowly augmenting fashion and the inspiratory muscles follow suit. But the moment they begin to contract they stretch their own Gougr endings and dispatch

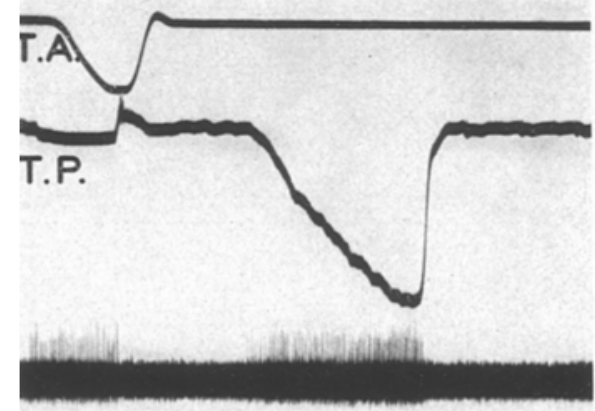

INTERNAL ARCUATE FIBERS

Fig. 73. Augmentation of GoLGI reflexogenic support of inspiratory contractions at a sensory station of the inspiratory reflex arc. [MAGEE, Bricker and Gesell: Amer. J. Physiol. 129, $415(1940)$

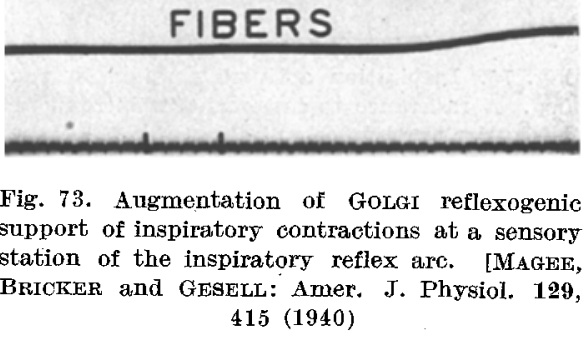
the signals so generated through the sensory circuit back to the reticular cells. The reflexly intensified discharge whips up the ventral horn cells and so the muscles, to greater activity, which in turn increases the output of Gougr signals impinging on the center. 
This self-augmenting cycle thus tends to steepen the gradient of the inspiratory discharge in a manner analogous to that described for the vagus nerve (see

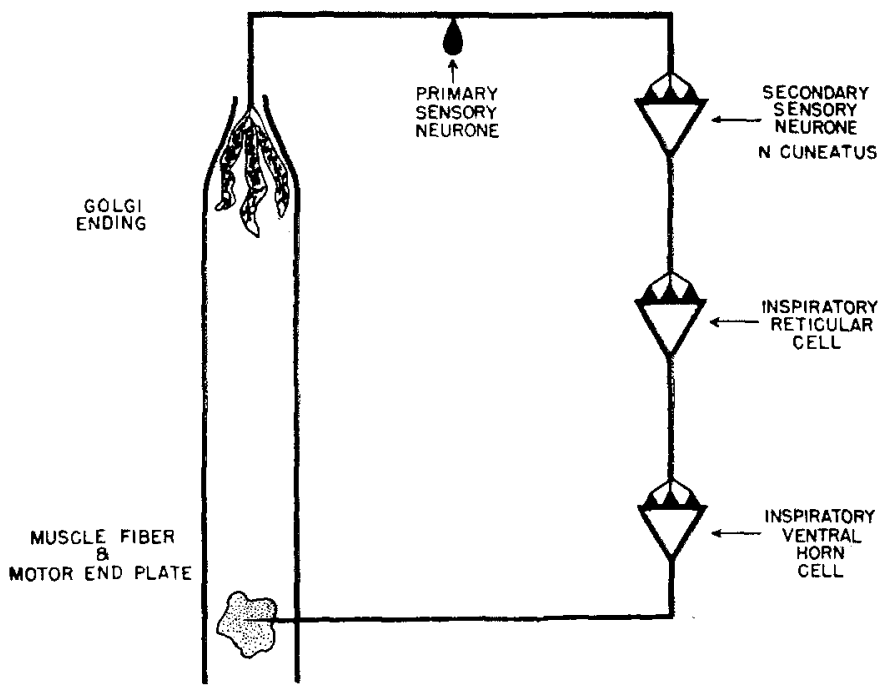

Fig. 74. An hypothetical neuromechanical circuit of GolGI reflexogenic support of respiratory contraction. Muscular contraction increases the activity of the GoLGI endings which increases the activity of the corresponding reticular cells etc. [WonzNLAK and Gesedx: Amer. J. Physiol. 126, 568 (1939).]

fig. 174). As Hess (90) and Fleisch (99) have stated, the automatic discharges of the respiratory center are but incompleted integrations. Only as

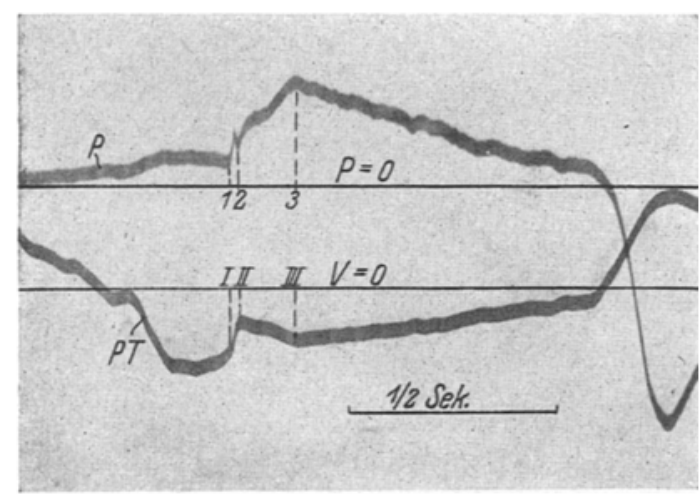

Fig. 75. Inspiration activating reflex in vagotomized dog. A resistance to inspiration is introduced at point pressure) curve rises up to point 2 and the stream velocity (P. T.) diminishes up to point II. Between points $2-3$ (II-III) an inspiration activating reflex develops which increases the stream velocity and the tracheal negative pressure. [Fueiscr: Eng. Physiol. 36, 249 (1934).] I-I. For purely mechanical reasons the $\mathrm{P}$ (or negative

the respiratory act unfolds it is corrected and adapted to the mechanical conditions which develop. The speed and accuracy of the resulting proprioceptive reflexes has been demonstrated by FuEISCH with the aid of his pneumotachigram (see fig. 75). For example, an introduction of resistance to the movement of air during inspiration and expiration increases the strength of the inspiratory and expiratory contractions respectively and thus tend to maintain a constant flow of air regardless of the resistance encountered. Conversely, reduction of the resiConversely, reduction of the resi-
wer of the inspiratory and expirastance to the flow of air decreases the power of the inspiratory and air.
tory contractions and again tends to maintain a constant flow of air. It is only reasonable to expect that shorter ares also exist which connect the Gougr endings with the ventral horns directly through the cord. We 
should so interpret the recent results of FuErsch and TrIPoD (100) who have demonstrated the existence of corrective respiratory reflexes after transection of the cord, vagi and dorsal cervical roots.

\section{The Reflexogenic Support of the Muscle Spindles.}

If the stresses and strains of the spindles of a contracting muscle are removed as the muscle shortens [Fulton and Pi Suner (96) and Matthews (13)] and if the signals arising in the spindles are inhibitory, it follows that inhibition must be removed in an inspiratory muscle in direct proportion to the degree of its shortening. Should this mechanism actually prevail it will be noticed that it is the only instance of direct reflex inhibition which we have so far presented. Even so, the reflex need not be regarded solely as a purely inhibitory phenomenon for contraction may actually be reinforced by a removal of the initial inhibition. The expiratory muscles would, however, be stretched and thereby inhibited thus allowing efficient reciprocating activities. In accordance with this concept we tentatively connected the muscle spindles of the inspiratory and expiratory muscles to the inhibitory poles of the inspiratory and expiratory reticular cells respectively. Similar connections were made with inspiratory and expiratory motor cells in the cord (see fig. 47).

If it be true that an inspiratory contraction is augmented by an automatic removal of a direct inhibitory reflex, what can be the purpose of a direct inhibitory reflex, particularly since the Gougr and muscle spindle reflexes would seem to serve the same purpose? We are inclined to regard the muscle spindle reflex as primarily protective and only secondarily employed for the purpose of drive. For example a powerful flexor contraction meeting insurmountable resistance, driven all the harder by the increasing GoLGi reflex, might lead to rupture were it not for the opposing reflex initiated by the protective stretching of the muscle spindles.

\section{A Comparison of the Respiratory Act with Rhythmic Motor Activities of the Spinal Cord.}

For a number of years Graham T. Brown (101 and 102) has called attention to a similarity of respiration and the rhythmic activities of the cord. According to him, both sets of motor integrations are coupled activities, in which opposing muscles are under the control of half centers reciprocally interconneeted. These interconnections not only accomplish the necessary reciprocal inhibition, but play a part in the rhythmic turning of each phase of contraction as well. If we may accordingly think of inspiratory contractions as representing flexor activity and expiratory contraction as extensor activity, much may be gained by a further comparison of the respiratory act with the spinal cord activity, for each similarity that can be established adds that much basie material for the interpretation of the respiratory act. 
One important point in common to breathing and the rhythmic activities of the cord is their elicitation by a continuing or steady type of stimulation.

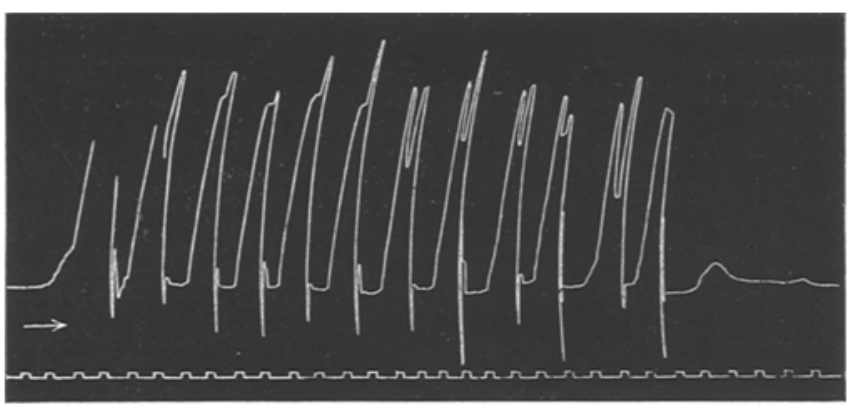

Fig. 76. Rhythmical leg movements of a decapitated frog whose legs are immersed in a weak solution of sulphuric acid. [MAROKWALD: Z. Biol. 5, 149 (1887).]

Thus we call attention to the elicitation of "rhythmic movements of the leg of a decapitated frog which had been immersed in 0,5 per thousand solution of sulphuric acid" by Marckwald (34) (see fig. 76). The invariable rhythimic response of the scratch reflex to such stimuli as high frequency electrical shocks, galvanic current and a beam of light as demonstrated by Sherrington is seen in fig. 24. The elicitation of

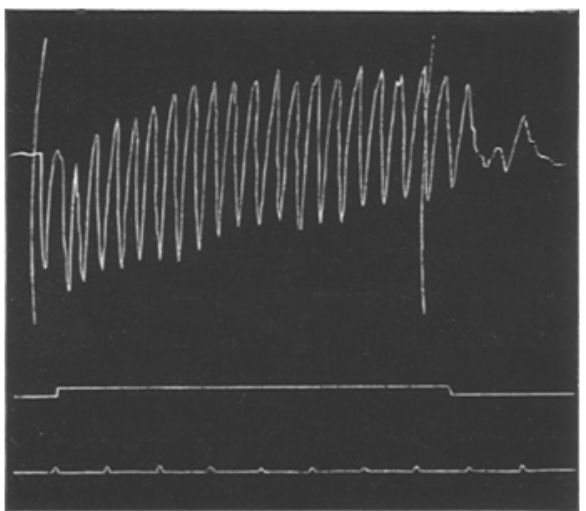

Fig. 77. Crossed stepping-reflex (spinal dog) elicited by unipolar faradization of the opposite foot. The flexions of the limb succeed each other at a rate of about five times in two seconds-about half the frequency they exhibit in the scratchreflex. The "after-discharge" of the reflex includes four "steps", the last being of small amplitude and slow, as with the final beats of the seratch-reflex. Time marked below in seconds. Above the time line is the signal line, showing the period of application of the stimulus. (SHERRINGTON: Integrative Action of the Nervous System. New York: Scribner's Sons 1906.)

duces about hale the respiratory stimalation of that produced by injection into both carotid bodies. A strong electrical stimulation of Herings nerve or of the central end of a cutaneo sensory nerve, such as the saphenous, activating a large number of fibers produces greater hyperpnea than does weaker stimulation. The results are similar to those of graded stimulation of the scratch reflex illustrated by 
Sherrington in fig. 24. As in the spinal reflexes, both frequency and strength of breathing increase with increasing strength of excitatory stimulation.

We have seen that signals, regardless of their origin, add their effects to those of others which impinge upon the excitatory end of common neuron. PAIN and GoLgi signals may add their excitation to those of the chemoceptor, thus increasing the sum total of reflexogenic drive of the respiratory neuron. Such complementing action of widely differing receptors was recognized by NAGEL (103) (1894) in the reflexes of the actinian. Surface receptors, one receptive for tactual stimuli, the other for certain chemical stimuli coexist in the tentacles and the reflexes elicited through combination of mechanical with chemical stimuli seem to combine harmoniously and mutually reinforce each other. SHERRINGToN remarks that in the case of the flexion reflex the receptive field includes not only reflex arcs arising in the surface field, but proprioceptive reflex arcs arising in the depth of the limb which mutually reinforce each others action. This is nothing more than a substantiation, in the lower spinal centers, of the principle of synaptic drive advanced for the respiratory neurons, namely that the activity of a discharging nerve cell is primarily related to the sum total of excitatory impulses impinging on the cell, rather than to a temporal arrangement of impingement.

We have referred to the marked after-discharge continuing after the end of stimulation of the central end of Herings nerve (see fig. 26). That phenomenon is common to all respiratory reflexes and is unmistakably seen following the end of stimulation of the saphenous nerve in fig. 84. The degree of after-discharge varies with the intensity and duration of sensory stimulation both for the respiratory and lower spinal reflexes. That is evident on comparison of Sherringtons findings on the scratch reflex (fig. 25) and the respiratory records just described.

While it is true that the theoretically denervated respiratory center and the deafferented cord seem capable of a rhythmic activity of their own, both rhythms, as just observed, are nevertheless capable of gross modification by artificial sensory stimulation. Stoppage of breathing by either a predominantly inspiratory stimulus such as chemical stimulation of the carotid body or by a powerful expiratory stimulus such as stretching of the lungs during the expiratory phase represent extreme examples of such change. Fig. 45 illustrates a prologation of inspiration produced by intracarotid injection of cyanide in a vagotomized dog. Fig. 79 shows a smaller effect produced by intravenous injection. Prolonged expiratory tetanus from pulmonary inflation was shown in fig. 60 and 68. Electrical stimulation of any nerve capable of producing powerful expiratory activity will produce identical effects whether that stimulation be continuous as in sustained pulmonary inflation or whether it be an interrupted stimulus, such as seen in fig. 78 (Hamruton and GESELL (104)]. In this instance four groups of faradic stimuli were delivered 
to the central end of the superior laryngeal nerve with a resulting increased expiratory effect following each stimulation. Upstroke represents inspiration.

It is concluded from such well-known results as have just been enumerated that prolongation of either inspiratory or expiratory activity will tend to decrease

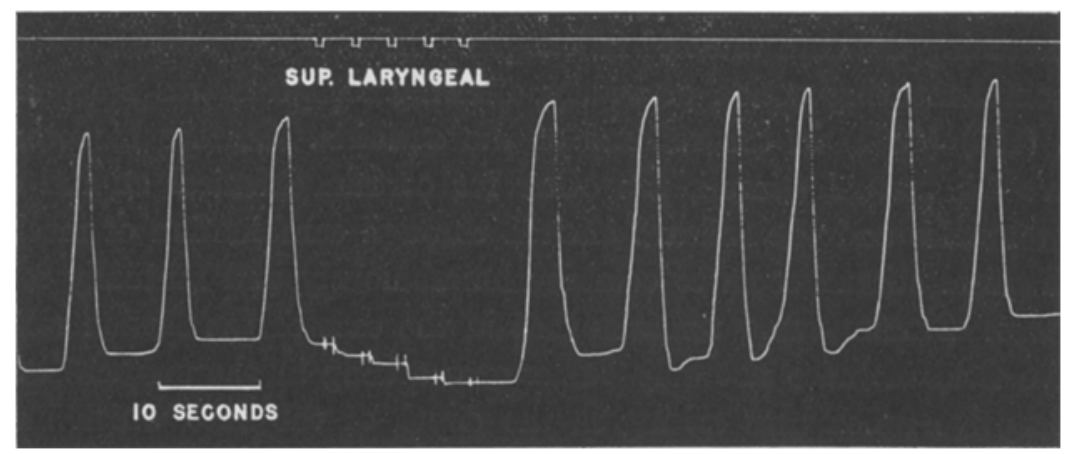

Fig. 78. Active expiration elicited by four groups of faradic stimuli applied to the central end of the superior laryngeal nerve of the dog. Upstroke represents inspixations. [HAMILTON and GEsELL: Amer. J. Physiol. 129, 373 (1940).]

the frequency of breathing. Following a combined line of reasoning it might seem as logical to conclude that simultaneous application of the inspiratory and expiratory stimulation, each of which produce retardation of breathing,

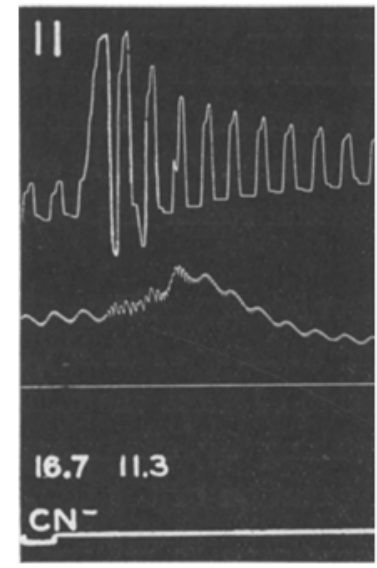

Fig. 79.

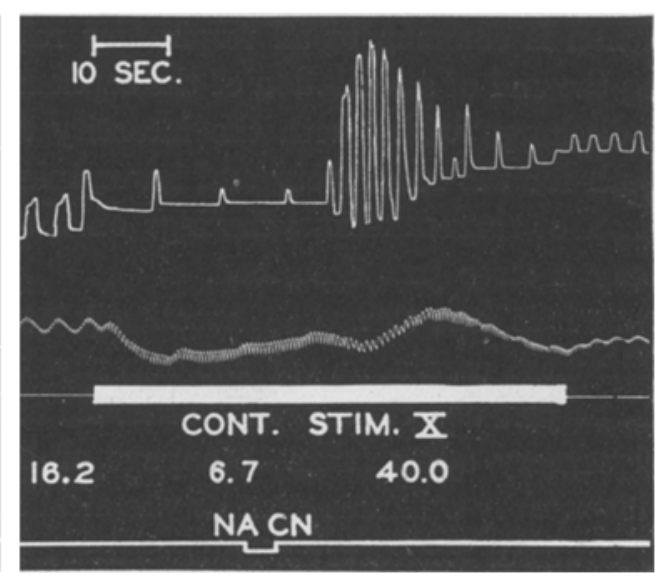

Fig. 80.

Fig. 79 and 80. Retardation of breathing resulting from intravenous injection of sodium cyanide in a vagotomized dog (fig. 79) and retardation of breathing resulting from afferent stimulation of the vagus nerve (fint part of fig. 80). Cyanide injected during vagal slowing now produces the so-called "paradoxical acceleration" of GESEL, STEFFENSEN and BROOKHART, i. e. one slowing effect added to another slowing

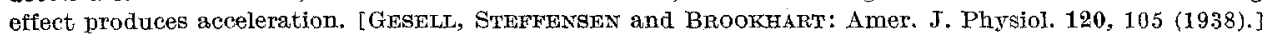

would produce a still greater retardation. The results are diametrically opposite [Geseil, StefFensen and Brooknart (105)]. An example of such findings is shown in figures 79 and 80 . The dog is vagotomized. Cyanide injection by itself will be seen to slow the frequency of breathing from 16,7 to 11,3 per 
minute primarily due to a prolongation of inspiration. Likewise, electrical stimulation of the central end of the vagus nerve by itself is seen to slow the frequency of breathing from 16,2 to 6,7 as a result of a prolongation of the expiratory phase. When cyanide was next injected during a period of slow breathing produced by vagal stimulation the frequency of breathing suddenly increased from 6,7 to 40 , when the cyanide reached the carotid body. The duration of inspiration and expiration are each reduced in time even though both are increased in depth. This constitutes a most elementary and beautiful example of the interplay of two steady state stimuli combining to produce a most effective type of breathing. It is furthermore significant that the artificial condition here supplied are very similar to those obtaining during oxygen lack in which the chemical stimulus, like cyanide, provides the necessary force to call forth deep inspirations and the deep inspirations by stretching the pulmonary proprioceptive endings provide the expiratory drive supplied by electrical stimulation of the vagus nerve.

Unable at the time to appreciate the significance of the production of acceleration by the combination of two retarding effects we called the phenomenon "paradoxical acceleration". But at the present moment any other result must seem paradoxical to us, for with the aid of our newer conceptions of breathing we can visualize the center acting like a pendulum between two springs, one, the in-

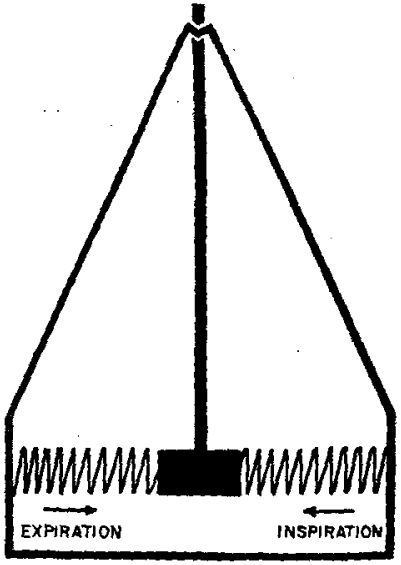

Fig. 81. A figurative diagram of the rhythm control of breathing in which the swing of the pendulum to the right is expiration and the swing to the left is inspiration. The mechanical forces of the springs pushing the pendulum from one side to the other are to be compared with the electronic forces of the balf centers (Grselt: Unpublished.) spiratory force, pushing it to the left and the other, the expiratory force, pushing it to the right (see fig. 81). If these forces are strong and evenly balanced, the pendulum will vibrate with great force and with a frequency differing from its own inherent rhythm. If the forces are weak and evenly balanced the pendulum will swing more gently and slowly at its own period in accord with its length. Our problem is to develop the details of respiratory rhythm in harmony with electrochemical forces along the lines which we have attempted to develop figuratively with the aid of mechanical concepts.

Stimulation of the superior laryngeal nerve or vagus increases the electrotonic excitation current of the expiratory cells because these nerves have copious expiratory comnections. This of itself stiffens the expiratory spring and pushes the pendulum to the right. At the same time the stimulation reduces the electrotonic exciting current in the inspiratory half center through reciprocal inhibition which is comparable to a softening of the inspiratory spring. The pendulum thus swings still further to the right. The same reasoning 
may be used for a predominantly inspiratory stimulation such as arises in the chemoceptors of the carotid body.

How then may other effects be tentatively explained, such as the accentuated expiratory action produced by the addition of the stimulation of the saphenous nerve to that of the superior laryngeal nerve in fig. 82 ? The pendulum (mechanical or electrochemical) is already to the right, due to the push of the superior laryngeal nerve. Upon excitation of the saphenous

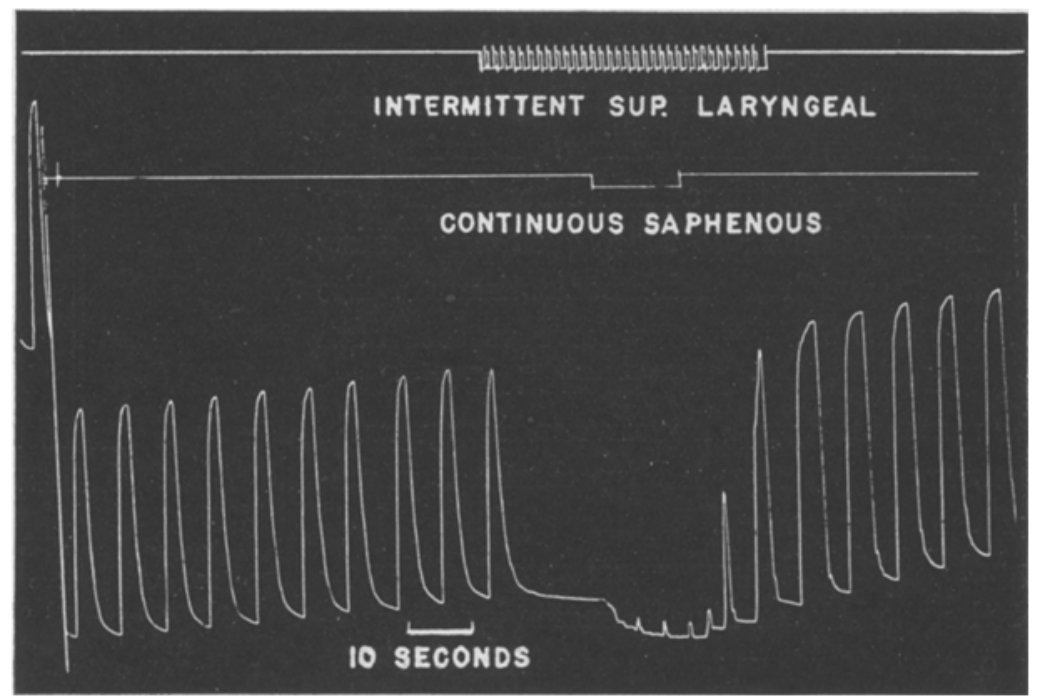

Fig. 82. Effects of saphenous stimulation when added to stimulation of the superior laryngeal nerve already in progress. Superior laryngeal stimulation elicits a weak expiratory contraction seen by careful inspection of the record which prevents the occurrence of inspiration. When the saphenous nerve is now stimulated, its expiratory component is added to the predominating expiratory reflex initiated by the preceding stimulation of the superior laryngeal nerve as indicated by the general depression of the record. [HAMILTON and Geseli: Amer. J. Physiol. 129, 373 (1940).]

nerve two additional forces are added -- one new push to the right, for the nociceptors are connected to the expiratory half center, and another new push to the left for the nociceptors are also connected to the inspiratory half center. The proof of this double push has already been presented in the increased depth of both expiration and inspiration upon stimulation of the saphenous nerve. The expiratory component of the saphenous nerve is thus added to the prevailing expiratory reflex or push of the superior laryngeal nerve and increases the compression of the chest. One force adds to the other just as the chemical stimulus of asphyxia which is capable of producing either inspiratory or expiratory activity was seen to add itself to the predominantly expiratory reflex evoked by pulmonary. inflation in fig. 68. Small inspirations break through as the chemical stimulus increases.

What happens if the pendulum is swinging on its own inherent rhythm as in fig. $83 \mathrm{~A}$ where both vagus nerves are cut and a series of faradic shocks are delivered to Henings nerve at regular intervals? Respiration can fall 
into phase with stimulation as it frequently does if the frequency of breathing and stimulation are approximately matched in which event the results are unsuitable for the present purpose. But failing to conform with the artificial rhythm of stimulation each group of shocks will fall in varying time relation with the respiratory cycle and thus introduce a varying set of conditions

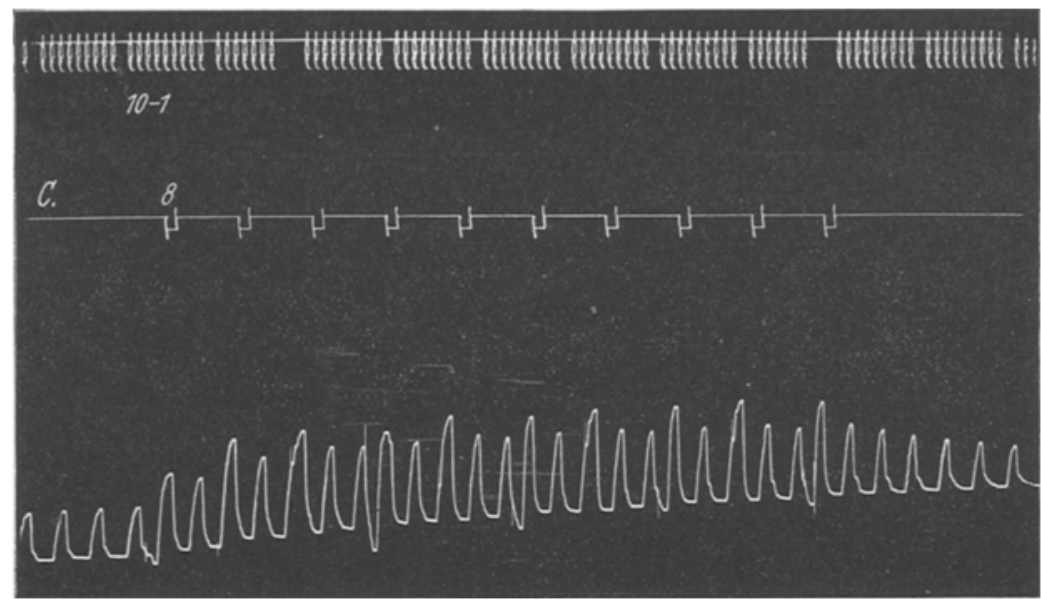

Fig. $83 \mathrm{~A}$.

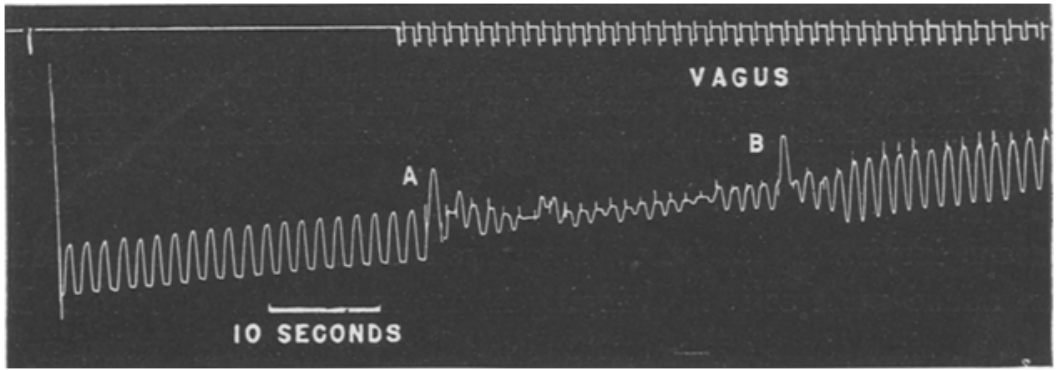

Fig. $83 \mathrm{~B}$.

Fig. 83. Effects of rhythmic afferent stimulation of BERIvas nerve and the vagus nerve in which stimulations fall in a hit or miss manner into varying positions in the inspiratory and expiratory phases. When stimulation accidentally falls into a favorable position in the inspiratory phase, the inspiratory component adds harmoniously to the existent inspiratory activity. When stimulation falls into a favorable position in the expiratory phase, the expiratory component adds harmoniously to the existent expiratory activity. [HAMILTON and Gesell: Amer. J. Physiol. 129, 373 (1940).]

such as presented in fig. $83 \mathrm{~A}$. To interpret the varying response to stimulation two points must be kept in mind. During the active phase of expiration the inspiratory half center is reciprocally inhibited and due to the low intensity of its electrotonic excitation current it is relatively unsusceptible to stimuli. The expiratory center is for the moment dominating the respiratory cycle and, therefore, is freely open to further stimulation. During the phase of active inspiration the conditions are reversed, the inspiratory half center is then open to further stimulation while the expiratory half center is barred. 
It will be seen at once that those stimulations falling with expiration actually increase the depth of expiration despite the fact that the chemoceptors are predominantly inspiratory excitatory in function. And those stimulations occurring during the inspiratory phase increase the intensity of inspiratory activity. Tuming to fig. $83 \mathrm{~B}$ in which the central end of the vagus nerve is stimulated one is impressed again with the irregularity of breathing. Particularly outstanding are the two deep inspirations at $A$ and $B$ representing harmonious additions of inspiratory activity to the central

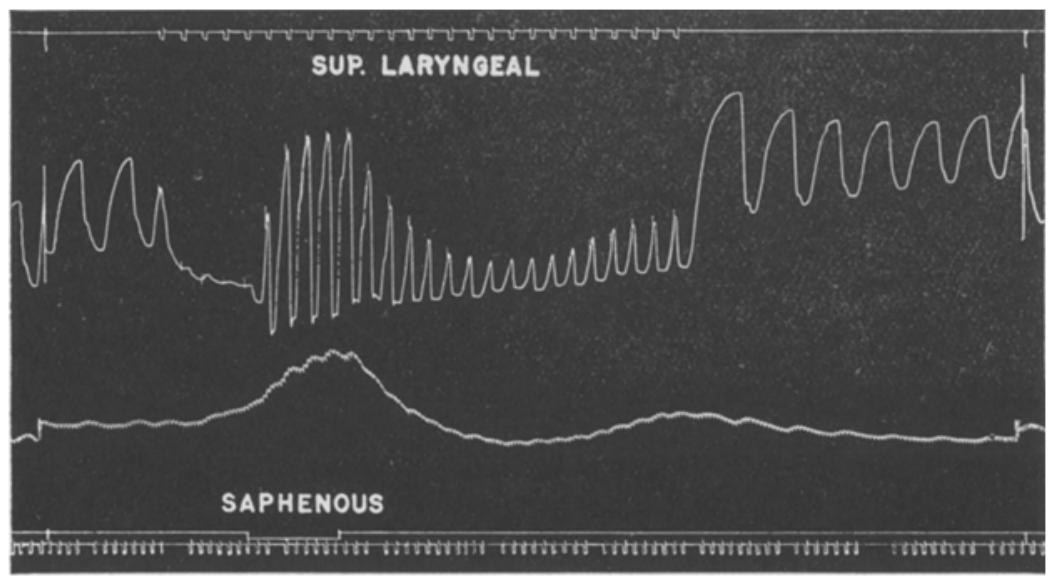

Fig. 84. A transformation of a sustained expiratory activity produced by a periodic stimulation of the superior laryngeal nerve into rhythmic breathing in phase with superior laryngeal stimulation by an additional continuous stimulation of the saphenous nerve. [Hammox and Gesell: Amer. J. Physiol. 199, $373(1940)$ ).]

spontaneous inspiratory activity. Expressed mechanically, the pendulum is given an added push as it is swinging in the inspiratory direction. Looking next for a possible cause of the series of slightly exaggerated expiratory excursions at the end of the record one sees that each vagal drive comes at the beginning of expiration, a suitable moment for pushing the pendulum in the expiratory direction. One is reminded of the need of "pumping the swing" at the right moment to accumulate the desired exhiliration.

The results which are obtained upon combined stimulation of nerves are by no means uniform e.g. in fig. 84 the addition of a continuous stimulation of the saphenous nerve to the expiratory action of rhythmic stimulation of the superior laryngeal nerve, not only accentuated the existent expiratory action, but brought about a rhythmic breathing in phase with the laryngeal stimulation. These results are most probably explained by the presence of a relatively strong inspiratory component in the saphenous nerve capable of initiating an inspiration between stimulations of the superior laryngeal nerve. As inspiration proceeds it becomes increasingly susceptible to interruption by the rhythmic expiratory stimulus of the superior laryngeal nerve 
which accomplishes that end. Expiration in turn becomes susceptible to interruption by the continuous inspiratory stimulation of the saphenous nerve etc.

If we have interpreted the literature correctly there are two mechanisms of inhibition, one arising through the reciprocating connections in the manner described by Brown and the other arising more directly by simple

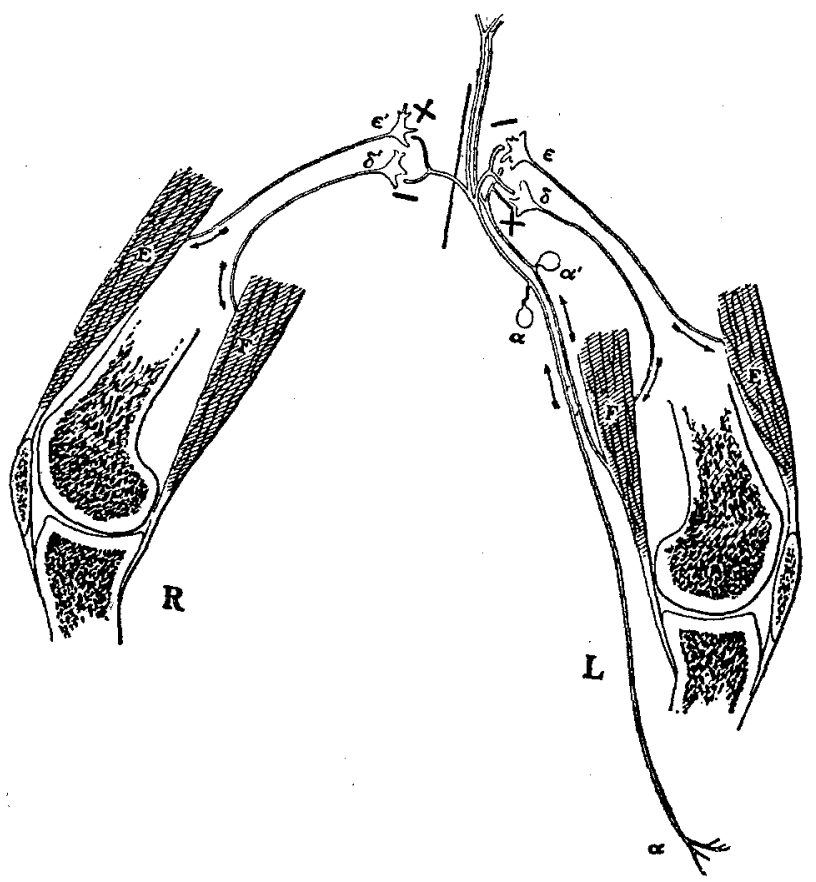

Fig. 85. Diagram indicating connections and actions of two afferent spinal root-cells, a and $a^{\prime}$, in regard to their reflex influence on the extensor and flexor muscles of the two knees. a, root-cell afferent from skin below knee; $a^{\prime}$, root-cell afferent from flexor muscle of knee, $i$. e., in hamstring nerve; e and $e^{\prime}$, efferent neurones to the extensor muscles of the knee, left and right; $g$ and $g^{\prime}$, efferent neurones to the flexor muscles; $\mathrm{E}$ and $\mathrm{E}^{\prime}$, extensor muscles; $\mathrm{F}$ and $\mathrm{F}^{\prime}$, flexor muscles. The "Schaltzellen" (v. MoNakow) probably between the afferent and efferent root-cells are for simplicity omitted. The sign + indicates that at the synapse which it marks the afferent fibre $a$ (and $a^{\prime}$ ) excites the motor neurone to discharging activity, whereas the sign-indicates that at the synapse which it marks the afferent fibre a (and $a^{\prime}$ ) inhibits the discharging activity of the motor neurones. The effect of strychnine and of tetanus toxin is to convert the minus sign into plus sign. (ShERrington: Integrative Action of the Nervous System. New York: Scribner's 1906.)

reflex action. Although the former is well accepted the second is also regarded as important. Sherrington believes that the more direct simple reflex inhibition builds on to the central reciprocating mechanism. Such reflex inhibition is always reciprocal, thanks to the double connections depicted in fig. 85. The afferent fibers branching as they enter the cord deliver the impulses arising in individual muscle sensory endings to antagonistic motor cells in the cord. One branch excitatory and the other inhibitory, permits excitation of one muscle group and a corresponding relaxation of the other. It is particularly on this issue that we differed with SHerrington in attempting to explain so called vagal inhibition of breathing for we prefer to adopt the 
principle of reflex drive, in which power operates the integrating mechanism with relatively little use of brakes. An arrangement of this kind would allocate but one function to most receptors, namely that of drive. The muscle spindle reflex, to which we have already referred, may be an exception more apparent than real due to its special protective use. The sinus stretch reflex would be another exception (see page 600), again more apparent than real, for it is unrelated to the fundamentals of motor integration proper.

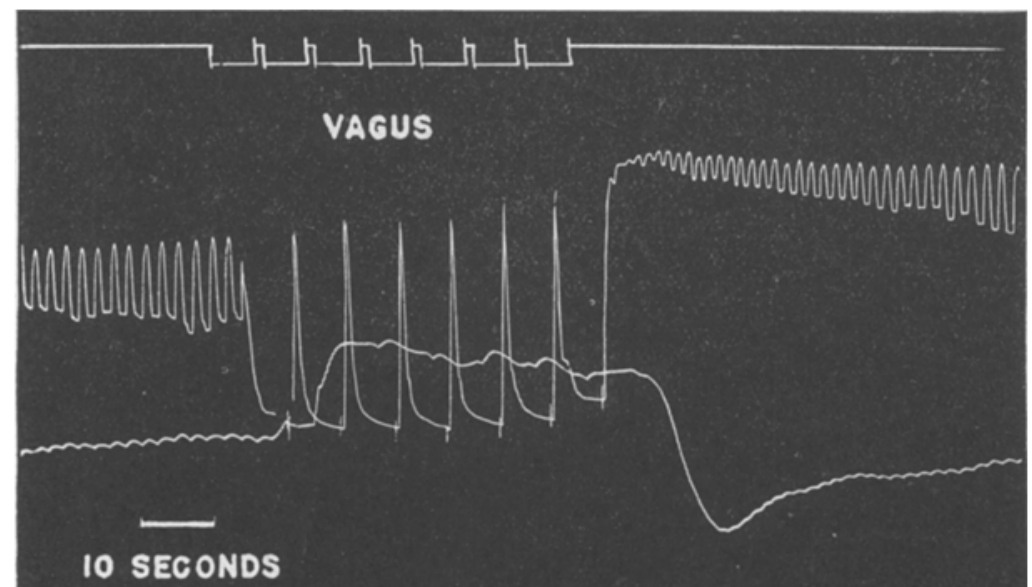

Fig. 86. Effects of prolonged and strong periodic central stimulations of the vagus nerve interrupted by short periods of no stimulation. Each stimulation produces a powerful expiratory compression of the lungs followed by an abrupt inspiratory contraction. The powerful inspiratory contraction appearing during the silent periods of stimulation has been interpreted as rebound. The results are to be compared with those of Sowton and SHerringron in fig. 87. [HAMILTON and Geseli: Amer. J. Physiol. 129, 373 $(1940) .1$

We now come to a most interesting record, fig. 86 , in which stimulation of the central end of the vagus nerve produces powerful expiratory contractions followed immediately by powerful inspiratory contractions at the cessation of each stimulation. Directly underneath this figure we have placed a strikingly similar series of muscular contractions, fig. 87, labeled "Knee-Extensions by Rebound: Isolated Extensor Muscle" which represent the results on the spinal reflex recorded by Sowton and Sherrington (107).

If we were to attempt an explanation of rebound it would arrange itself about the reciprocal interconnections of the half centers. Stimulation of the vagus nerve would be pictured as firing synapses on both inspiratory and expiratory neurons but only the expiratory cells would discharge due to the fact that the expiratory phase is in progress, therefore, reciprocally inhibiting the inspiratory cells. If now we assume that change, as well as the strength of electrotonic current, is important in exciting the axon hillock it becomes apparent that a cessation of activity of the expiratory half center must have an excitatory effect upon the inspiratory half center. This was mentioned 
in our introductory discussion of the workings of the reciprocating connections. It was concluded that one center not only inhibited the other but in that process actually excited itself by reducing the inhibitory action of its opposing center. In this mechanism we see an explanation of rebound. By suddenly withdrawing its inhibitory potentials from the axon hillocks of the inspiratory cells at the close of expiratory activity, the expiratory half center thereby actually increases the electrotonic current in the cells of the inspiratory half center. The sudden increase of electrotonic current thus discharges these cells.

Finally we must consider Hammoudas and WILsons views on the control of the frequency of breathing. It is necessary to recall that they insist upon the existence of a specific acceleratory nerve whose function presumably is that of controlling frequency of breathing. These fibers are thought to differ from ordinary cutaneo sensory nerves, which are also known to produce acceleration of breathing when electrically stimulated. Our main argument against the views of HAMmoUda and WIL-

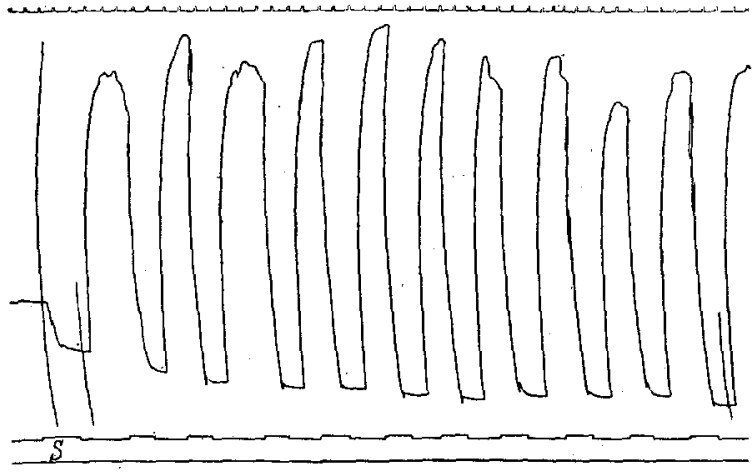

Fig. 87. Knee-Extensions by Rebounds: Isolated Extensor Muscle. On applying the electrical stimulation (first rise of the signal line) to the foot-nerve, the myogram line falls and remains down during the stimulation, showing the reflex inhibition of the tonus. After three seconds the stimulus is discontinued (fall of the signal line); a post-inhibitory rebound contraction ensues during this discontinuance of the stimulation. This contraction is then cut down by reapplication of the stimulus (second rise of signal line), the muscle being again relaxed by inhibition. On withdrawal of this stimulation a rebound contraction reensues. This is repeated eleven times, each lapse of the stimulus giving an active extension of the knee. Decerebrate cat. Time marked above in seconds. SowTon and SHERRINGTON. (SHERRINGTON: Selected Writings of Sir CHARLES SHERRINGTON. New York: Hoeber 1940.)

son is our belief that sensory

accelerator nerves, pure and simple, are non-existent, not merely for respiration but for any rhythmic nervous integration such as the scratch or stepping reflex. We rather look upon rhythm as a balance between the activity of half centers which in turn is dependent upon the individual intensities of stimulation of the half centers. Until that conception fails it would seem more advisable to interpret the results of HAmmouda and WiLson on graded cold block, to coincidental changing proportions of inspiratory and expiratory excitatory components.

In that connection it may not be amiss to suggest why stimulation of cutaneo sensory nerves is often so effective in producing a marked increased frequency of breathing, even with the vagi blocked. The inspiratory and expiratory components of cutaneo sensory nerves are often well balanced. In contrast, Herings nerve though èqually excitatory is predominantly inspiratory. It, therefore, is effective in creating a high frequency of breathing 
only when properly combined with an expiratory component from other sources. Recovery from a strong inspiration may be very prolonged indeed (see fig. 88).

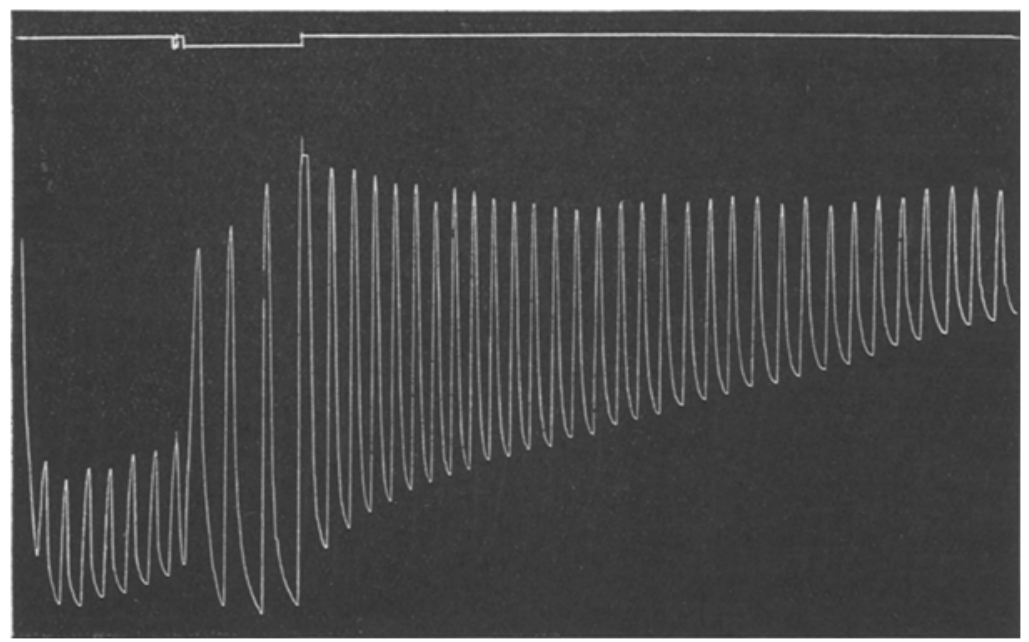

Fig. 88. Faradic stimulation of HERINGs nerve produce very deep inspirations. Expirations are increased to a smaller extent. Note that the frequency of breathing was diminished. This may be due to the prolonged recovery following each deep inspiration. (Geseil, Hamuton and Brassrield: Unpublished.)

\section{The Individual Localization of the Inspiratory and Expiratory Centers.}

The evidence indicating the location of the primary respiratory center in the reticular substance of the lower medulla has already been presented. In that discussion we commented briefly on the diffuse structural arrangement of the center and the structural intermixture of the inspiratory and expiratory half-centers. The functional division into twin centers - the inspiratory and expiratory half centers has been considered in much greater detail. At this particular moment we are concerned with the delimitation of these individual centers, not so much in relation to neuro anatomy itself, but rather in relation to physiological principles which have recently become involved [see Prtss, Magoun and Ranson (27, 108, 109)].

Marckwald $(34,110)$ was among the first to be interested in this problem. In attempting to elucidate the mechanism of inspiratory spasms, now more commonly known as apneusis, he stimulated the medulla electrically. The following are some of his comments.

"If, after the medulla oblongata had been successfully divided above the respiratory centre, the vagi were cut in the neok, so that respiratory spasms appeared, and if then the medulla was stimulated rhythmically or continuously with intermittent shocks, one was always successful in modifying the respiratory spasms and in liberating rhythmic respirations." - "Although on the whole, direct stimulation of the medulla oblongata brought forth more inspiratory movements, yet I often saw such plain and active expirations appear that I could not daubt the existence 
of an expiratory center of respiration as well as an inspiratory one." - "The most important fact gained by direct stimulation of the respiratory center is that the center can only be electrically stimulated in the same way as if the stimuli came from the centripetal nerves and, therefore, we stimulate only the sensory ganglionic part of the centre with intermittent current."

The italics are ours.

Examples of his findings are illustrated in fig. 89, 90, 97, and 98. Fig. 89 shows how periodic breathing is transformed into regular and more

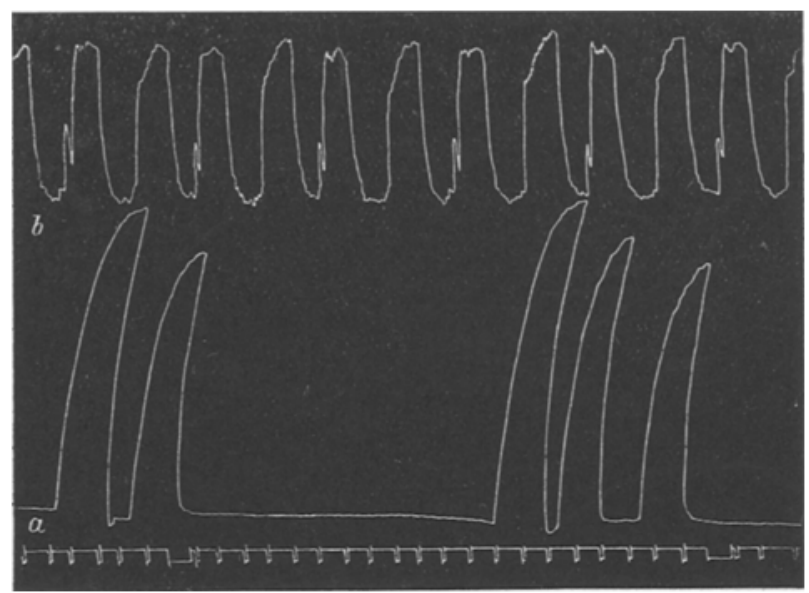

Fig. 89. Lower tracing A shows irregular slow periodic breathing which is transformed into regular breathing by faradic stimulation of the medulla as shown in the upper treacing B. [MARCKwald: Z. Biol. 5, $149(1887)$. ]

frequent breathing by faradic stimulation of the medulla, whether the stimulation be continuous or rhythmic. Normal breathing is accelerated by similar

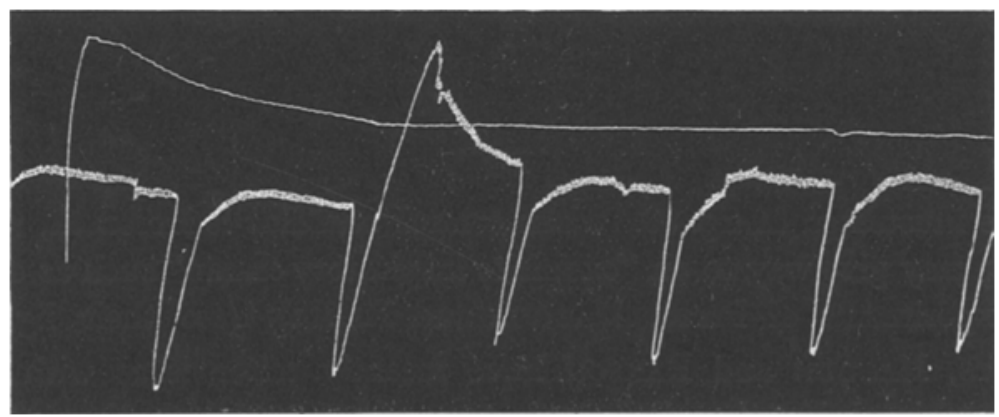

Fig. 90. The rhythmical breathing shown in the lower tracing is produced by faradic stimulation of the medulla after combined transection of the brain above the respiratory center and ragotomy: The upper record is an inspiratory spasm in which stimulation is missing. [MARCKwald: Z. Biol. 5, 149 (1887).]

stimulation. Marckwald then compares the similarity of these results with those obtained by stimulation of sensory nerves. Inspiratory spasms produced by transection of the medulla and vagus nerves are also interrupted by continuous or rhythmie stimulation (see fig. 90 and 97). The smooth, long, drawn out curve in fig. 90 shows the prolonged inspiratory tetanus resulting 
from the transections and the lower curve shows how these spasms are interrupted by electrical stimulation of the medulla. Similar interruption of inspiratory spasms by continuous faradic stimulation of the superior laryngeal

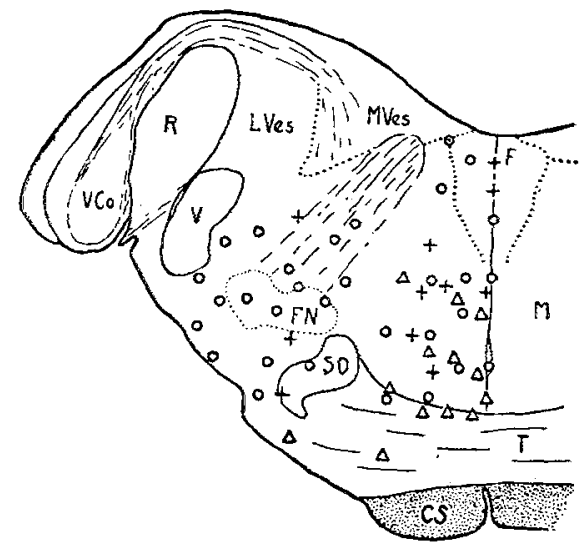

A

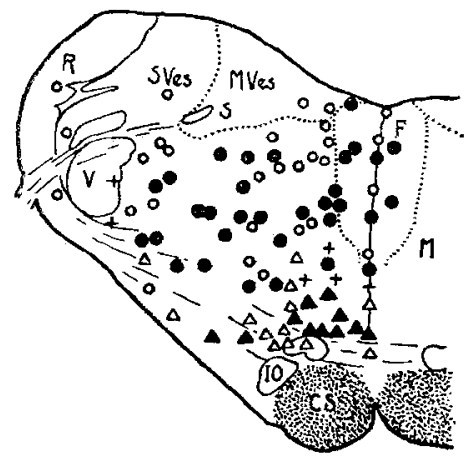

C

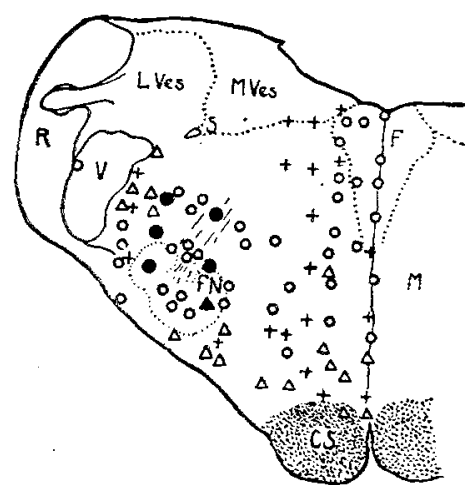

B

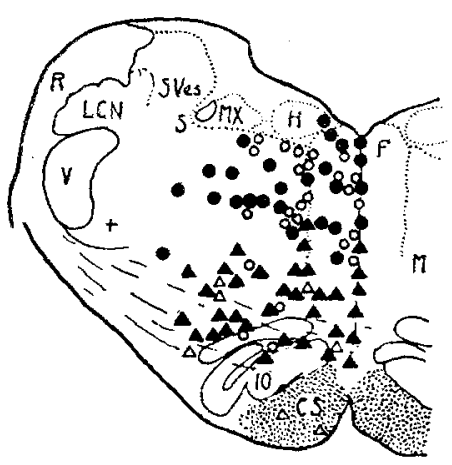

D

Fig. 91 .

nerve are illustrated in fig. 98. When central stimulus is increased, rhythmic breathing may give way to an "exquisite inspiratory cramp" such as described in the experiments of Pitts, Magoun and Ranson (see their fig. 107 A).

Pitrs, Magoun and Ranson have recently taken issue with MarckWALD in maintaining that the respiratory responses which they obtain on stimulating the medulla are not due to the stimulation of sensory structures, but to direct stimulation of the center itself. Their results may be summarized in their own words and figures.

"Many varieties of respiratory responses were obtained. In fact if one considers all the possible combinations of increases, decreases and no change, in rate, amplitude, midposition etc., each can be found in the medulla, and an attempt to indicate localization would lead to confusion and detract from the more precise localization of maximal reactions. Consequently we have chosen to consider the 2 types of response shown in fig. $1 \mathrm{~A}, \mathrm{~B}$ and $1 \mathrm{E}, \mathrm{F}, \mathrm{G}$ (see our fig. 107) as maximal types if the apnea maintains without interruption for the duration of the test 
stimulus, i.e., 12 to 18 seconds or more and if, in the case of inspiration the overinspiration amounts to $75 \mathrm{cc}$. or more, and if in the ease of expiration the position of rest is in at least as great a degree of expiration as that of normal respiration preceding stimulation."

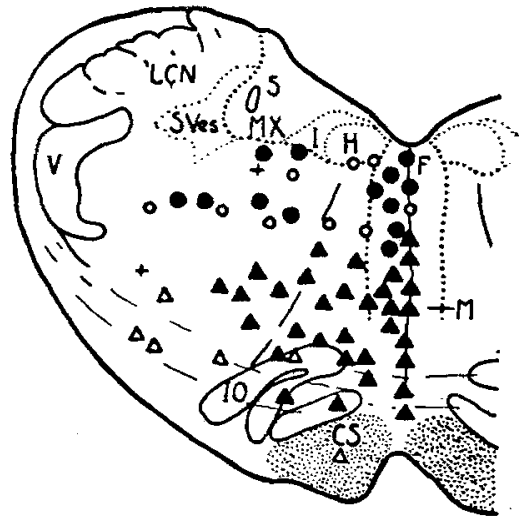

$E$

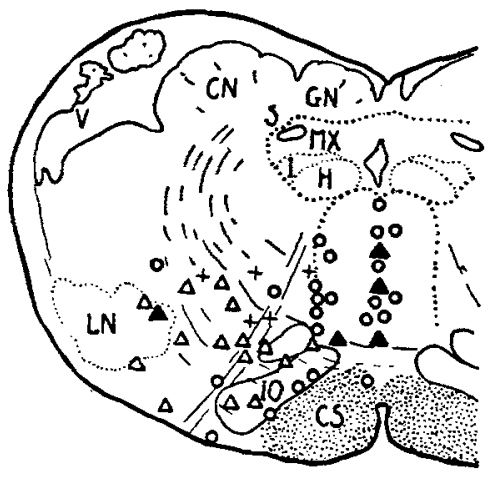

G

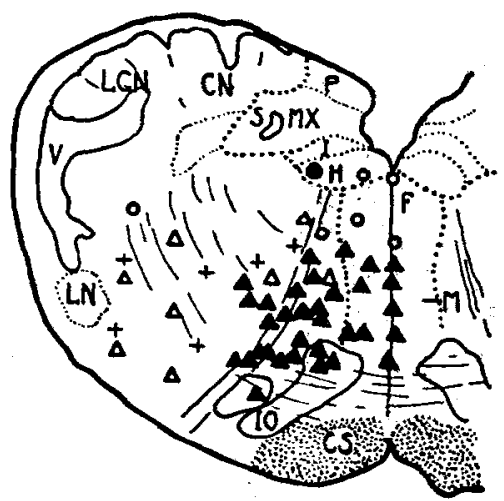

$\mathrm{F}$

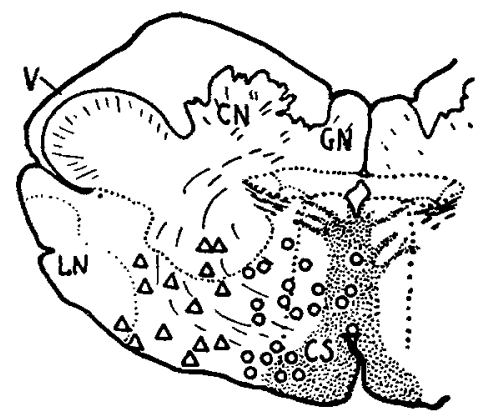

$H$

Fig. 92.

Figs. 91 and 92. Sections of the medulla of the cat taken at $1 \mathrm{~mm}$. intervals from $2 \mathrm{~mm}$. above the cephalic end of the inferior olive to $1 \mathrm{~mm}$. below the candal end of the inferior olive. Between sections $G$ and $H$ is an interval of $2 \mathrm{~mm}$. The plane of the sections is sloping downward and forward 15 degrees from the verticle coronal plane of the HorsLeY -Clarke coordinates. The relation of these sections to surface anatomy of the medulla is shown on the right side of fig. 17. Solid circles, maximal expiratory reactions; solid triangles, maximal inspiratory reactions; hollow cireles, submaximal expiratory reactions; hollow triangles, submaximal inspiratory reactions; plus signs, midpositional changes, i. e., reductions in amplitude or apnea. Cuneate nucleus, CN; corticospinal tract, CS; medial longitudinal fasciculus, F; gracile nucleus, GN; hypoglossal nucleus, $H$; intercallate aucleus, I; inferior olive, IO; lateral cuneate nucleus, LCN; lateral reticular nueleus, $L N$; lateral vestibular nueleus, L ves; medial lemnisens, M; motor nucleus of vagus, MX; medial vestibular nucleus, $M$ ves; area postrema, $P$; restiform body, $R$; tractus solitarius. $S$; spinal vestibular tract and nucleus, $\mathrm{S}$ ves; spinal tract of the trigeminal nerve, $\nabla$; ventral cochlear nucleus VCo. [Pitrs, Magoun and Ranson: Amer. J. Physiol. 126, 673 (1939).]

"The location of these maximal inspiratory and expiratory responses can be briefly sum. marized as being in the reticular formation of the medulla in the region over the inferior olive, the expiratory region lying dorsal to, slightly cephalic to, and cupped over the cephalic end of the inspiratory region which itself lies as a plate over the dorsal surface of the upper four-fifths of the inferior olive (see fig. 17). Rough calculations indicate that the inspiratory and expiratory regions occupy each about $30 \mathrm{cu} . \mathrm{mm}$. of the reticular formation. It must be emphasized that 
these maximal responses are obtained from no other region of the brain stem than that shown in fig. $3 \mathrm{~B}, \mathrm{C}, \mathrm{D}$ and $4 \mathrm{E}, \mathrm{F}, \mathrm{G}$ (our fig. 91 and 92). Our exploration has extended from a level in front of the hypothalamus to the cervical cord."

They add

"It must be emphasized that with a stimulus intensity of 8 volts at' 240 stimuli per second there is little spread of current beyond the tips of the electrodes. In fact we routinely observed in certain regions a complete reversal of response from expiration to inspiration on moving the tips of the electrodes $1 \mathrm{~mm}$."

It will be well for the reader to visualize the exact requirements of deliminating a mass of $30 \mathrm{cu} . \mathrm{mm}$. into separate inspiratory centers. This mass if spherical would have a diameter of approximately $3 \mathrm{~mm}$.

At the same meeting in which Pitts, Magoun and Ranson made their preliminary report, BrookHART (111) described his results obtained with low voltage stimulation, 0,5 to 1,5 volts, in which he was unable to elicit characteristic responses indicative of a selective stimulation of either the inspiratory or expiratory center. Like the Northwestern workers, he too, got every variety of respiratory response with weak stimulation. This also agrees with MaRoKWaLD who elicited tetanus only with powerful stimulation. The reason given by BrookHART for employing low voltage stimulation was to avoid the spread of current which must, if it occurs, invalidate an exact localization such as attempted by Pitrs, Magoun and Ranson. To simplify his analysis of results BrookHaRT divided his responses, more or less arbitrarily, into eight groups, differentiated according to rate and amplitude changes during the stimulation and recovery periods. The distribution of the points from which these types of responses were obtained is summarized in a series of histograms one for each type of response (see fig. 93). The following is quoted from his final paper (112).

"In the upper right hand corner of each histogram indicating the type of response is a small drawing of the respiratory changes characteristic of the response. The percentages of the total number of responses obtained from each individual structure which fell into the various groups of responses are indicated along the left hand margin of each histogram. Each bar represents a nuclear group or fiber tract as indicated at the bottom of the figure. Along the bottom edge of each histogram are numerals indicating the actual number of responses involved in the computations of the percentages."

"From these graphs it may be seen that no single structure gave rise to only one type of response. It is clear also that no single type of response was elicited from any one structure and from no other."

Brookнart, therefore, concluded that the low voltage stimulations of the brain stem fail to reveal the existence of anatomically separated inspiratory and expiratory centers.

Since then three final reports have been published on this subject by Pitts, Magoun and Ranson $(27,91,92)$ in which they retain their original contentions without discussion or citation of the work of BrookHart. Funton (112) has accepted the findings and conclusions of the high voltage experiments but apparently has overlooked the low voltage experiments. For these 

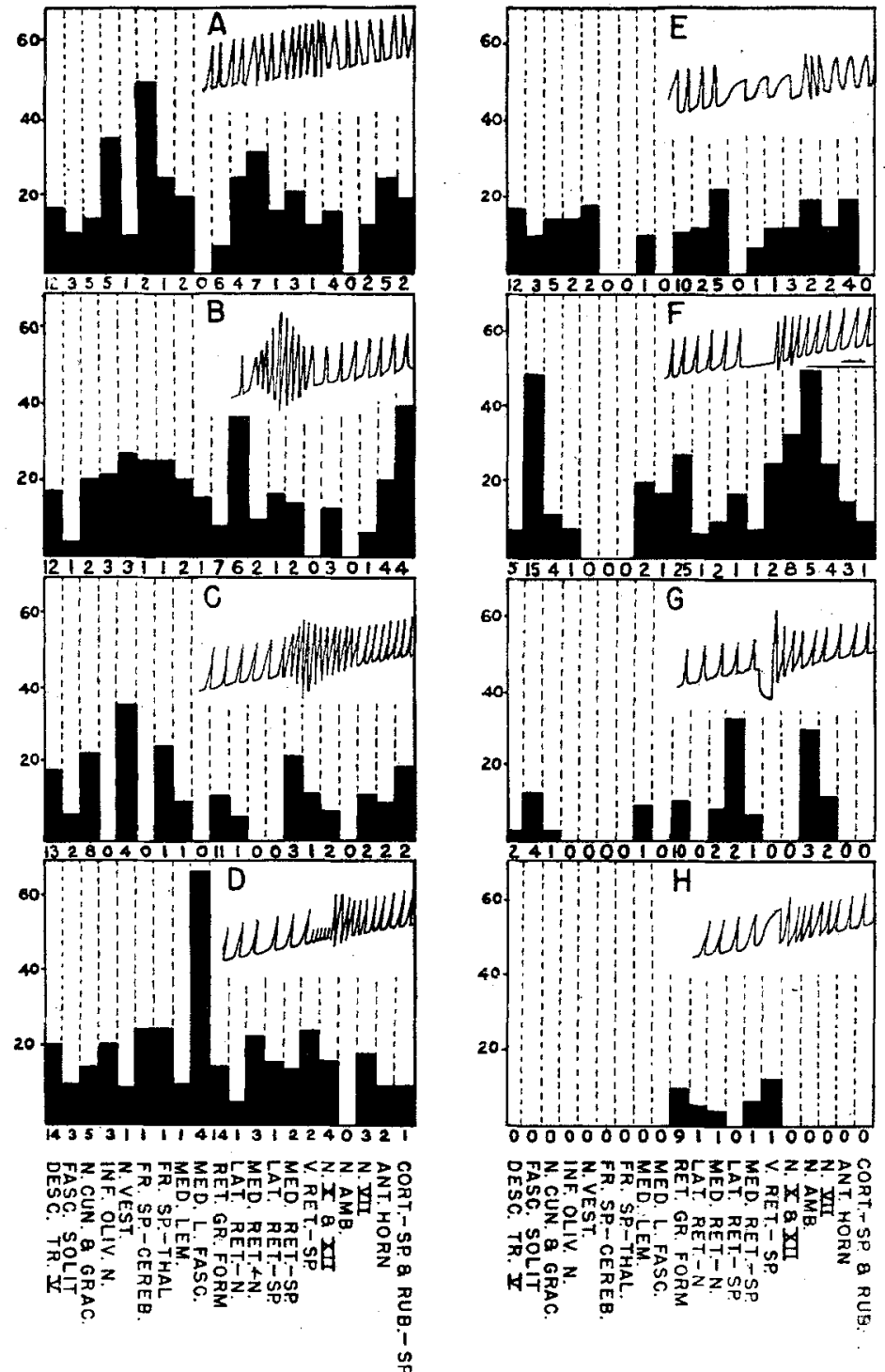

Fig. 93. Histograms indicating the relative frequeneies of occurrence of any single type of response in the individual medullary formations. The type of response represented by each histogram is indicated by a small spirometer tracing in the upper right hand corner of each graph. The percentage of the total number of responses elicited from any single formation which fell into each type group is indicated along the left edge of each graph. The number of responses involved in the computation of the percentage for each structure is shown along the bottom of each graph. At the bottom of the figure are the names of the medullary formations represented.

Abbreviations: Desc. Tr. $\nabla$ : Nucleus of and descending tract of the trigeminal; Fasc. Solit.: Fasciculus Solitarius and its nucleus; N. Cun. and Grac.: Nuclei Cuneatus and Gracilis and related fasciculi; Inf. Oliv. N.: Inferior olivary nucleus, interolivary fibers, and olivo-cerebellar fibers; N. Vest.: Nuclei and fibers of the eighth nerve; Tr. Sp.-Cereb.: Dorsal and Ventral spino-cerebellar tracts; Tr. Sp.-Thal.: Lateral and ventral spino-thalamic tracts; Med. Lem.: Medial Lemniscus; Med. Lo. Fasc.: Medial longitudinal fasciculus; Ret. Gr. Form: Reticular gray formation; Lat. Ret. N.: Lateral reticular nucleus; Med. Ret. N.: Medial Reticular Nucleus; Lat. Ret.-Sp.: Lateral reticulo-spinal tract; Med. Ret.-Sp.: Medial reticulo-spinal tract; V. Ret.-Sp.: Ventral reticulo-spinal tract; N. X and XII: Dorsal efferent nucleus of $\mathrm{X}$ and nucleus of XII; N. Amb.: Nncleus Ambiguus; N. VII: Nucleus facialis and its fibers; Ant. Horn: Cells of the Ventral horn; Cort-Sp. and Rub.-Sp.: Lateral Cortico-spinal and rubro-spinal tract and fibers of the pyramidal decussation; Pyram. Decus.: Pyramidal decussation; Corp. Rest.: Corpus restiformis; N. Sup. Oliv.: Superior Olivary nucleus. [BRookhart: Amer. J. Physiol. 129, 709 
reasons it seems desirable to attempt an explanation of the difference of opinion now existing on this phase of the subject. In our opinion the strength of Brookmarts experiments rests with the precautions which he took against current spread, the accurate anatomical location of the effects which he elicited, and the superiority of his electrodes. It will be recalled that he placed tiny lesions of about $1 \mathrm{~mm}$. in diameter at each site of stimulation and then retested the effects of stimulation. If stimulation still elicited a change in breathing that observation was discarded. High voltages were purposely avoided because of the danger of current spread. Pitrs, Magoun and Ranson made no direct check of this kind. They localized their site of stimulation by following the track which their coarse electrodes produced, a method impossible with the much finer needle electrodes used by Brookнart. They base their assumption of no current spread upon an argument rather than on a direct control. They state

"The magnitude of the maximal responses obtained is worthy of emphasis. The inspiration on stimulation of the inspiratory center may amount to over $200 \mathrm{cc}$., often over 10 times the tidal air. This response is obtained by stimulation of less than $3 \mathrm{cu} . \mathrm{mm}$. of the reticular formation, for with voltages as high as 20 volts spread of stimulus is less than $1 \mathrm{~mm}$. judging from reversal or disappearance of reaction on moving the electrodes $1 \mathrm{~mm}$. Since the volume of brain substance from which maximal responses may be obtained is over $30 \mathrm{cu}$. $\mathrm{mm}$. and since the direct excitation of less than $3 \mathrm{cu}$. $\mathrm{mm}$. within this region can yield responses which appear to be maximal, it is probable that the center is fired as a whole through rich synaptic interconnections. Evidence to be presented in a succeeding paper confirms this view."

Since making his preliminary report BrookнаRт has repeated the high voltage experiments of Pitts, Magoun and Rasson on the dog. The results of these experiments are shown in fig. 94 and discussed in his own statement. "Inspection of fig. 94 will reveal, that at least as far as the dog is concerned, the regions reacting with inspiration and expiration are nowhere nearly as sharply defined and dissociated in the reticular formation as PitTs, Magoun and Ranson have found for the cat. Maximal expiratory and inspiratory responses are not confined precisely to the reticular formation and do not show the invariable dorsal-ventral distribution which has been reported. In many cases maximal inspiratory responses have occurred upon stimulation when the electrode tip was simply resting on the surface of the medulla without penetration. Likewise, in a number of cases maximal and submaximal responses of both types have occurred when the electrode tip was bearing on the bone ventral to the medulla. In addition, such responses have been elicited from regions rostral to, caudal to and lateral to the respiratory areas which have been described for the cat."

On gradual increase of intensity of stimulation from low voltages $(0,5$ to 1,5$)$ to high voltages ( 8 to 10 ) the sustained response was seen to develop from any one of the low voltage responses. "It was impossible to abolish the high voltage responses by lesion, even if the lesion was enormously larger than any which were placed previously (see fig. 95). In several instances, 

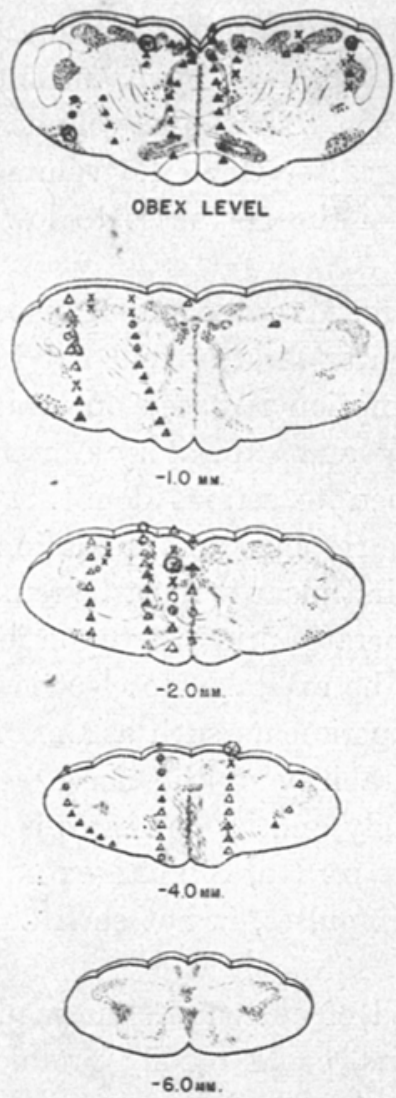

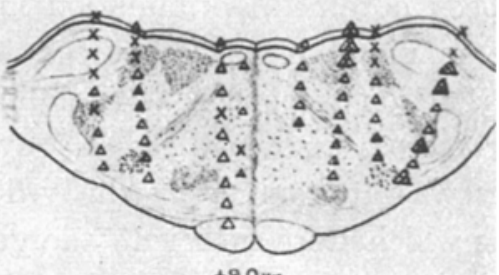

+9.0w:

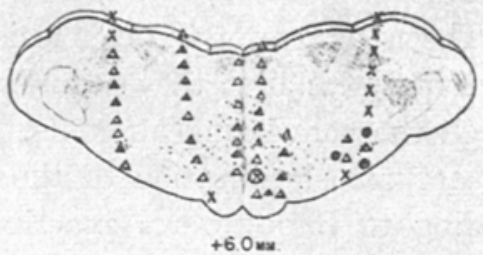

+60 wa

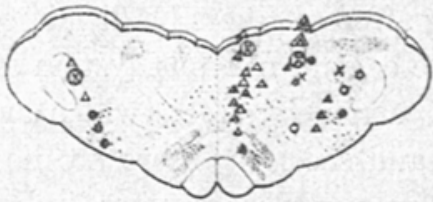

+3.0 was.

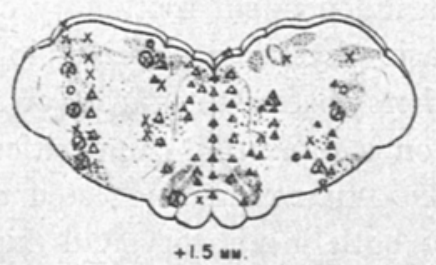

EXPIRATION

MAXIMAL A SUBMAXIMAL $\triangle$ MAXMAL O SUBMAXIMAL O

ALL OTHER RESPONSES $-x$

Fig. 94. Maximal and submaximal inspiratory and expiratory responses obtained by stimulation with high voltages. The depth of the stimulating tip of the electrode below the surface of the medulla is indicated in millimeters. [BRookrart: Amer. J. Physiol, 129, 709 (1940).]

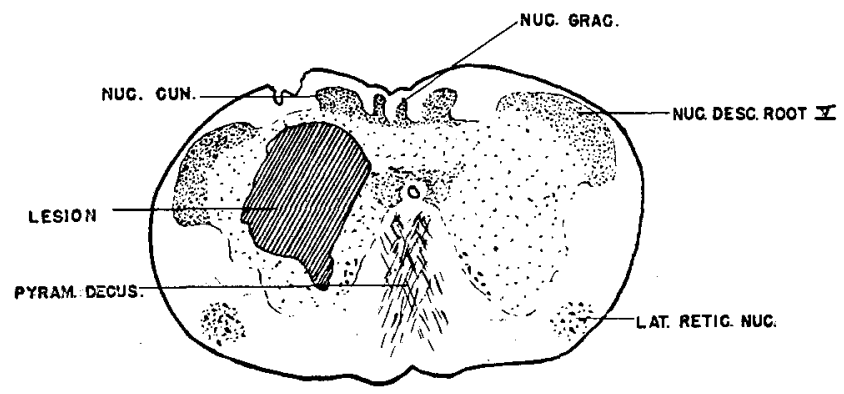

Fig. 95. Projection drawing of a lesion in the lower medulla which failed to abolish a maximal inspiratory response to a stimulus of 8,0 volts applied near the center of the lesion. [BrookHarT: Amer. J. Physiol. 129, $709(1940)$. ]

Ergebnisse der Physiologie. 43. 
such lesions were placed in the medulla at points from which maximal responses had been obtained. Stimulation in the center of such lesions continued to evoke maximal responses of equal and sometimes greater magnitude than the pre-lesion response. These lesions measured between two and four millimeters across and were roughly ovoid in shape. Thus, the volume of such lesions was approximately thirty-five times greater than the volume of the lesions which were sufficiently large to abolish the responses to low voltage stimulations."

The weakness of the conclusions of Pitts, Magoun and Ranson lies in their hypothesis that they stimulated the center directly and not indirectly through the converging afferents. This assumption is based on their supposedly accurate localization of the inspiratory and expiratory centers which according to BrookHarts experiments is open to serious doubt. But even granting the validity of their method, can the response to stimulation of converging afferent fibers be dismissed? In the first place it must be remembered that the central nervous system as a whole operates primarily upon impinging signals carried by fibers which are known to be excitable to electrical stimulation, and secondly there are no classical experiments such as ClaUde BerNARDs on muscle, proving independent irritability of the nerve cell body. Though independent irritability of the cell body and its dendrites is accepted as a basie physiological principle by Lorente de No, Sherrington, Gasser and others to explain effects of antidromic impulses, is not such acceptance based on a dangerous supposition?

Brookhart has suggested that a spread of current in the medulla, involving both inspiratory and expiratory units, could readily produce either a purely inspiratory or expiratory end resultant depending upon the predominance of either component. The force of that argument is supported in fig. 82 where the addition of saphenous stimulation to that of stimulation of the superior laryngeal nerve produced an expiratory response greater than that resulting from stimulation of the superior laryngeal nerve alone. That such effects are obtainable despite the existence of a strong inspiratory component in the saphenous nerve is most significant for it shows that a strong inspiratory or expiratory component could be concealed in the central stimulations described by Pitts, Magoun and Ranson. It has, therefore seemed probable that inspiratory effects produced by high voltage central stimulation have a high admixture of the expiratory component and vice versa and so cannot, for the present, be accepted as cleanly deliminating the inspiratory half center from the expiratory. Furthermore, if it is true that rich synaptic connections of the nature described by Pitts, Magoun and Ranson exist in the center which are capable of producing "circus impulse" one is puzzled to understand why the positively selective stimulation of BrookHART failed to reveal that fact. 
Through the courtesy of Dr. CRAIGIE who participated in the Respiration Symposium arranged by Dr. Henderson at the 1938 meeting of The Federation of Societies for Experimental Biology and Medicine, I am able to add his comment on the forces of neurobiotaxis in relation to the existence of anatomically distinguishable respiratory half ceñters.

"The most careful histological study has revealed no sign of a structurally differentiated respiratory centre. Now, there is in neuroanatomy a well-established principle called neurobiotaxis, according to which stimulogenously correlated neurons attract each other and tend to migrate towards each other during development, both phylogenetically and ontogenetically, thus bringing it about that cells of similar function become associated in definite architectural groups. The fact that no such group, even of the loosest kind, can be discovered in the location of the supposed respiratory centre is evidence against there being any such system of cells of closely similar function. Rather it would appear that the so-called centre must consist of rather widely scattered cells which, though they together exert a regulating effect on the motor mechanism of breathing, are excited by quite varied stimuli. Not improbably there may be considerable powers of vicarious functioning within this diffuse coordinating apparatus. Such a condition would account for the conflicting results reported by such a long series of physiological experimenters."

The electrical studies of Geselu, Bricker and Magee (12) have led them to agree with Craigre in the existence of a diffusely arranged center in which the inspiratory and expiratory centers may be rather intimately associated in an anatomical way. The reason for arriving at this hypothesis is that no location was found in the medulla in which the potentials were predominantly inspiratory or expiratory. Moreover a step-like penetration of the sounding electrodes about $0,1 \mathrm{~mm}$. at a time would often yield a series of respiratory potentials changing from inspiratory to expiratory and the reverse. Not infrequently one would record both inspiratory and expiratory potentials from one position of the electrode such as is seen for example in fig. $14 \mathrm{E}$ and $\mathrm{G}$.

In conclusion it may be stated that the problem of localization of the inspiratory and expiratory centers, should they exist as sharply deliminated structures, will be an extremely difficult one. Uncontrolled evidence cannot be accepted in a highly quantitative problem of this nature. The elicitation of either inspiration or expiration by central stimulation is no proof that the center has been directly stimulated for comparable results are obtainable by sensory nerve stimulation. Because an expiratory effect is produced by central stimulation is no proof that inspiratory nervous structures are not simultaneously stimulated, for mixed expiratory and inspiratory stimulation in sensory nerves is capable of producing an expiratory effect. We are, therefore, in agreement with the original conclusions of Marckwald that "the center can only be electrically stimulated in the same way as if the stimuli came from the centripetal nerves".

\section{Inspiratory Tetanus or Apneustic Breathing.}

One of the most interesting and illuminating of respiratory phenomena is the inspiratory spasm resulting from combined transection of the brain stem and vagus nerves. Brain stem transection or vago section alone may 
have little or no effect but when one is combined with the other regardless of the order of procedure "respiration changes in a very remarkable manner. The diaphragm immediately after extirpation of the vagi (or upper brain tracts) passes into a long inspiratory spasm, gradually not suddenly, as if

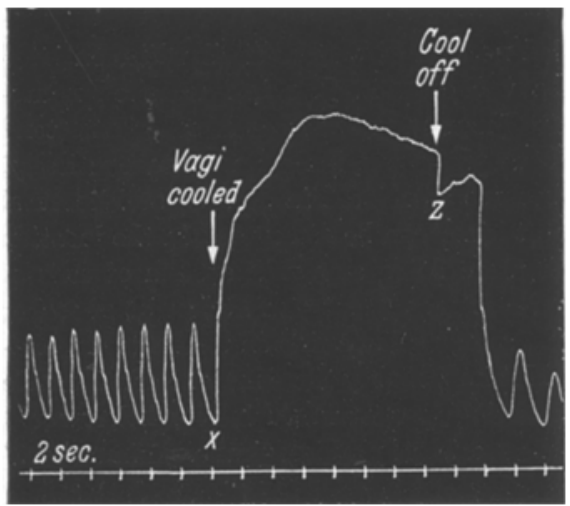

Fig. 96. Apneustic breathing produced by transection of the brain stem plus vagal block.

[STELLA: J. of Physiol. 93, 10 (1938).] an inhibition was removed. Thus the diaphragm in this spasm sometimes goes considerably beyond the ordinary height of an inspiration, and then falls back suddenly into the position of expiration" [MARCKWALD (34)]. An example of this old observation is seen in a recent figure from STELLA (96).

The frequency of breathing varies greatly under these experimental conditions. In some instances the spasmas may last a minute or more while in others ten seconds or less. So far as we are aware no explanation is yet available for these variations in rhythmicity. A most important observation of MARCKWALD (34) is the interruption of the inspiratory spasms by continuous faradic stimulation or rhythmic stimulation of the central end of either the

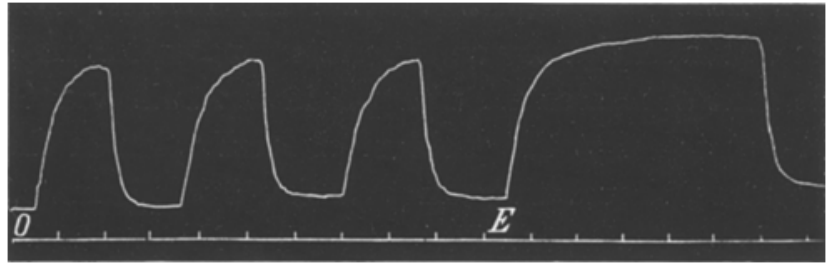

Fig. 97. Expiratory interruption of inspiratory tetanus by periodic faradic stimulation of the central end of the vagus nerves at 3 second intervals. Stimulation ends at $E$ whereupon the frequeacy of breathing is diminished. [MARCKwald: Z. Biol. 5, 149 (1887).] vagus or superior laryngeal nerves like that produced by similar stimulation of the substance of the medulla. The acceleration of breathing produced by faradic stimulation of the central end of the vagus at regular intervals is seen in

fig. 97. When stimulation is stopped the inspiratory contractions are more prolonged changing the frequency from 30 to 6 per minute.

By transecting the cord and cutting the dorsal cervical roots MarckWALD eliminated the impulses from the lower tracts as an essential factor in the production of inspiratory spasms. On the other hand he showed that animals with divided brain stem and sectioned vagus nerves were very susceptible to cutaneous stimulation which "quickens the liberation of different respiratory phases". As a result of his numerous observations Marckwald arrives at a number of pertinent conclusions:

"1. That the centers of respiration are situated in the medulla oblongata and are in close connection with the origins of the vagi. 
2. The respiratory centers in the medulla oblongata are automatically active, as well as excitable by reflex action.

3. The automatically active center can only liberate respiratory spasms, but no regular rhythmic respiratory movements.

4. Normal rhythmic respiration is a reflex act, mainly liberated by the vagi.

5. The vagi constantly stimulate; they possess tonus and are sufficient to serve as the only active regulators of respiration. During absolute rest of the animal organism they probably act alone.

6. Next to the vagi the upper brain tracts are of great importance for the liberation of regular rhythmic respiration. They are capable of replacing the non-activity of the vagi. During

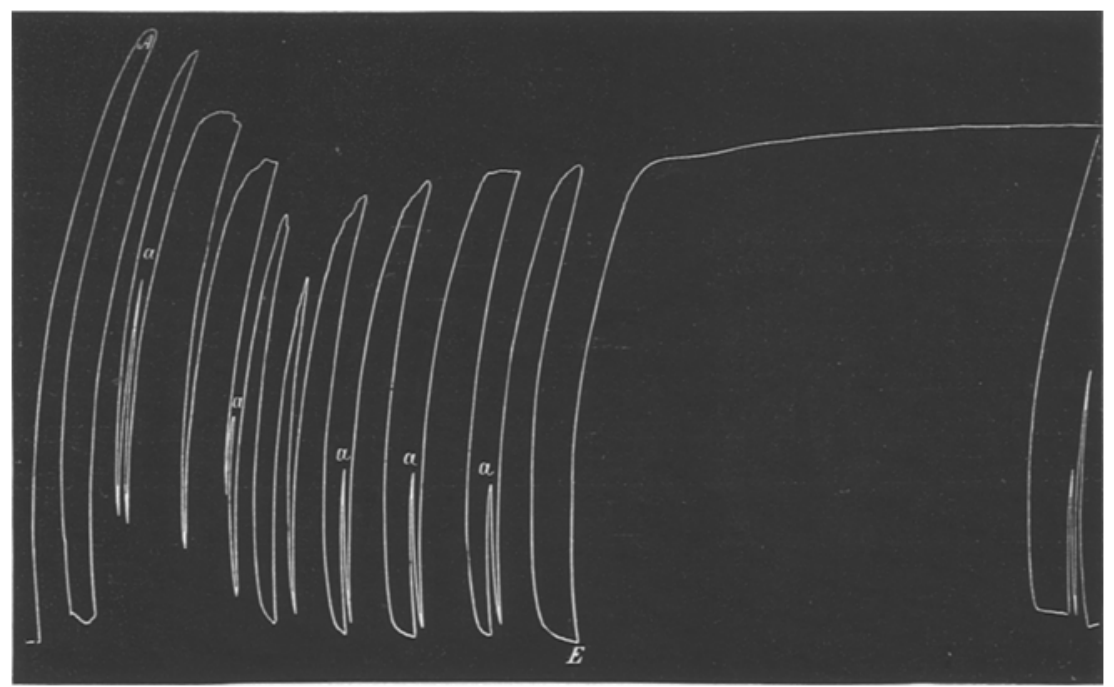

Fig. 98. Periodic interruption of inspiratory tetanus produced by faradic stimulation of the superior laryngeal nerve after double vagotomy. Inspiratory tetanus begins again at the end of stimulation at point E. [MARCEWALD: Z. Biol, 5, 149 (1887).]

sleep, during hibernation, or after certain narcotics the reflexes from the upper tracts on the respiratory center often remain alive. The great difference between the individual tracts lies in this, that whilst the vagi are in constant stimulation and act continuously on the respiratory center they also possess tonus; the upper tracts have only a secondary influence on respiration. From the brain flow all voluntary impulses of respiration, all emotions, all sensory impressions, and stimuli which are the result of mental actions. These all modify respiration.

7. There are no true respiratory centers above the primary centers in the medulla nor are there true respiratory centers in the cord."

The development of inspiratory spasms of relatively low frequency following division of the brainstem and vagi have been amply confirmed by many workers.

Of particular interest was the work of LumsDen $(114,115,116,117,118)$ in which inspiratory spasms or apneustic breathing (holding of the breath) was produced by various forms of chemical abuse such as asphyxia, chloroform etc. and by brain stem section. As a result of these experiments Lumsden believes that he has established the existence of four distinct respiratory centers (see fig. 99). 1. A gasping center just above the apex of the calamus scriptorius believed to be a persisting remnant of a primitive respiratory 
mechanism no longer playing a role in normal respiration. 2. An apneustic center at the level of the striae acousticae which when unregulated produced prolonged inspiratory spasms such as those described by Marckwald. 3. A

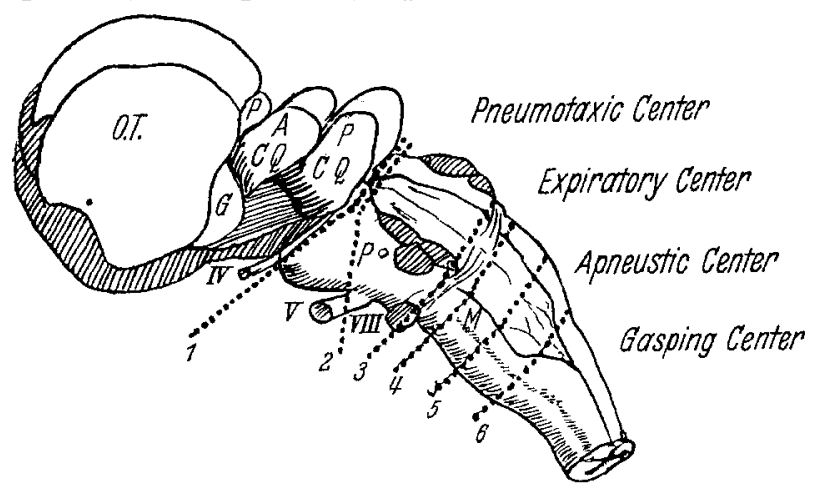

Fig. 99. Lumsdens respiratory centers. [Lumsden: J. of Physiol. $58,81(1923)$. pneumotaxic center located in the upper pons whose function it is to interrupt the apneustic center at regular intervals and thus establish the normal frequency and depth of breathing and 4. An expiratory center located between the gasping and apneustic centers which when the phase of expiration is active aids the pneumotaxic center in interrupting apneusis by bringing about an expiration. According to LUMSDEN however expiration is largely passive which would place the role of rhythmic interruption of breathing upon the pneumotaxic center. In our opinion the schema of LUMsDen is unnecessarily complex
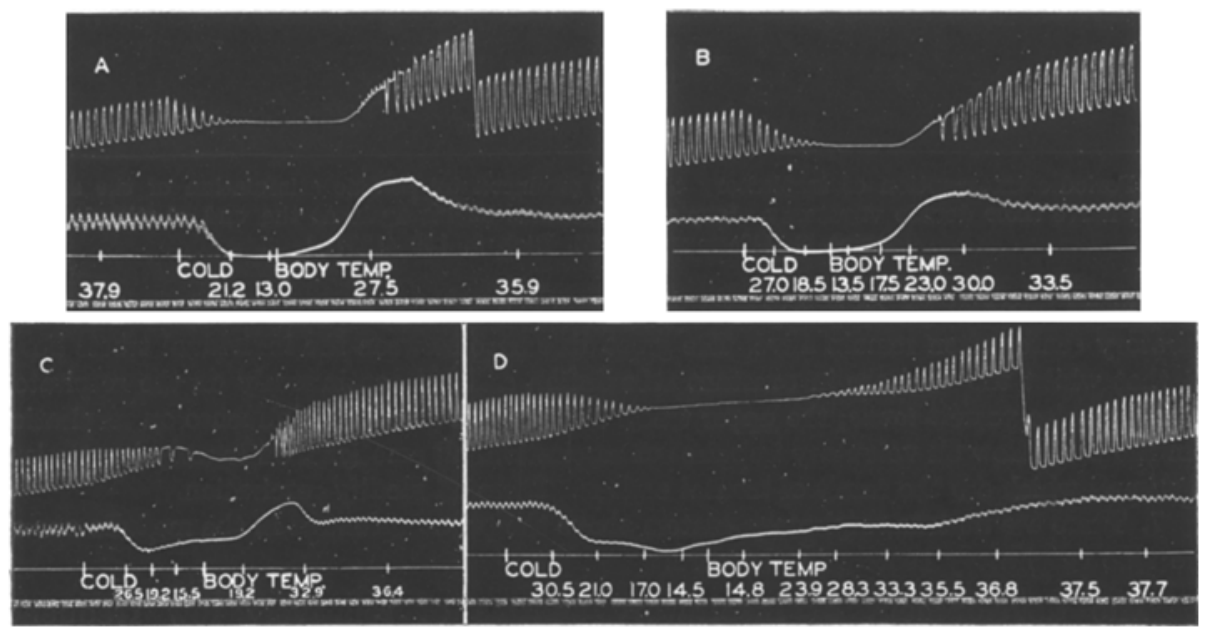

Fig. $100 \mathrm{~A}, \mathrm{~B}, \mathrm{C}$ and D. Cooling of the medulla in the region of the obex stops breathing. Apneustic breathing is seen in records A, B and C. [NIcholson: Amer. J. Physiol. 115. 402 (1936).]

when a simple concept of balance between two half centers seems to meet all present needs. We much prefer to subscribe to the simple principles layed down by Nroholson (119), Nicholson and Brezin (120), Nicholson and Sobin (121), Sobin and Nicholson (122) in their studies on the medulla.

They modified the activity of the respiratory centers by cold, heat, and chemical action. Their first experiments show the effects of temperature 
changes in the medulla, produced either by flooding the floor of the exposed medulla with chilled Lockes solution or by direct metallic contact of a specially devised cold applicator. Severe cooling in the region of the obex stopped breathing completely (fig. 100) from which it is concluded that the respiratory centers lie within the immediate vicinity of the obex. Slight or moderate local cooling of the floor of the fourth ventricle just anterior to the apex of

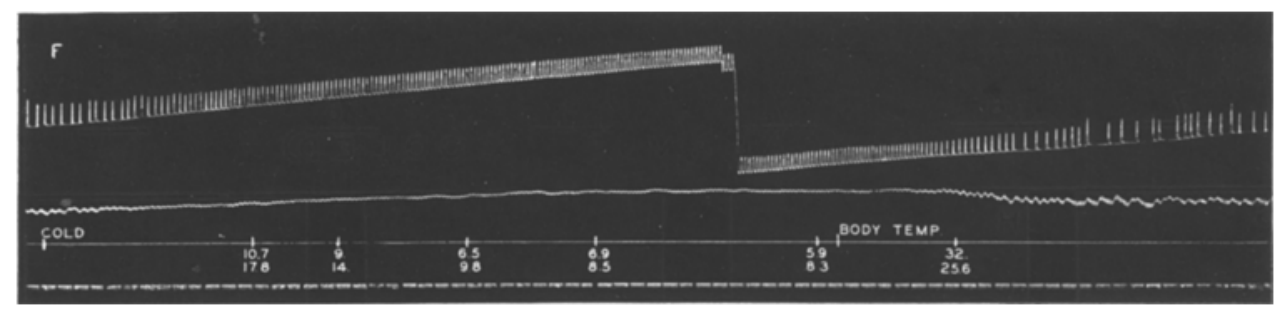

Fig. 101. Shortening of the expiratory phase of breathing by localized cooling of the medulla. Since the inspiratory phase remains unaffected the frequency of breathing increases. [NichoLson: Amer. J. Physiol. $115,402(1936)$.]

the calamus scriptorius depresses expiration leaving inspiration largely unaffected. This is seen in an acceleration of breathing produced by shortening of the expiratory pause (fig. 101). Acceleration is, therefore, most pronounced when the normal expiratory pause is long. Greater cooling eventually slows the frequency of breathing by a disproportionate increase in the duration

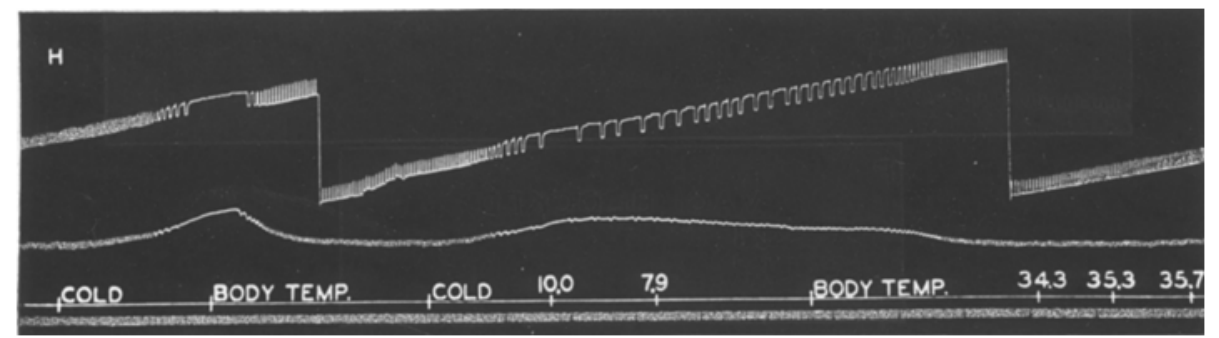

Fig. 102. Apneusis lasting as long as a minute produced by cooling of the medulla. [NrchoLsoN: Amer. J. Physiol. 115, 402 (1936).]

of the inspiratory act (fig. 102). "This effect might be so great as to cause inspiratory arrest for one to two minutes apparently exactly similar to the apneusis described by Lumsden."

Nicholson and Brezin next show that slight cooling of the medulla abolishes the retardation and acceleration of breathing produced by inflation and deflation of the lungs respectively (see fig. 103A). Sections $G$ and $H$ show the usual acceleration and retardation and $I$ and $J$ show the absence of the reflexes during cooling. The elimination of vagal impulses are, therefore, considered important in the establishment of apneusis by severe cooling of the medulla, but there is another factor as well. For example if the medulla is slightly cooled just enough to produce acceleration of breathing from reduction 
in the expiratory period, the addition of vagal block, which alone produces only slight effects upon breathing now produces marked apneusis. Nichouson and Brezis explain apneusis as follows. "Apneusis consists essentially in a more or less prolonged failure to interrupt inspiratory activity. It has been observed by Kroneoker and Marokwald (1880), Lumsden (1923) and Keller
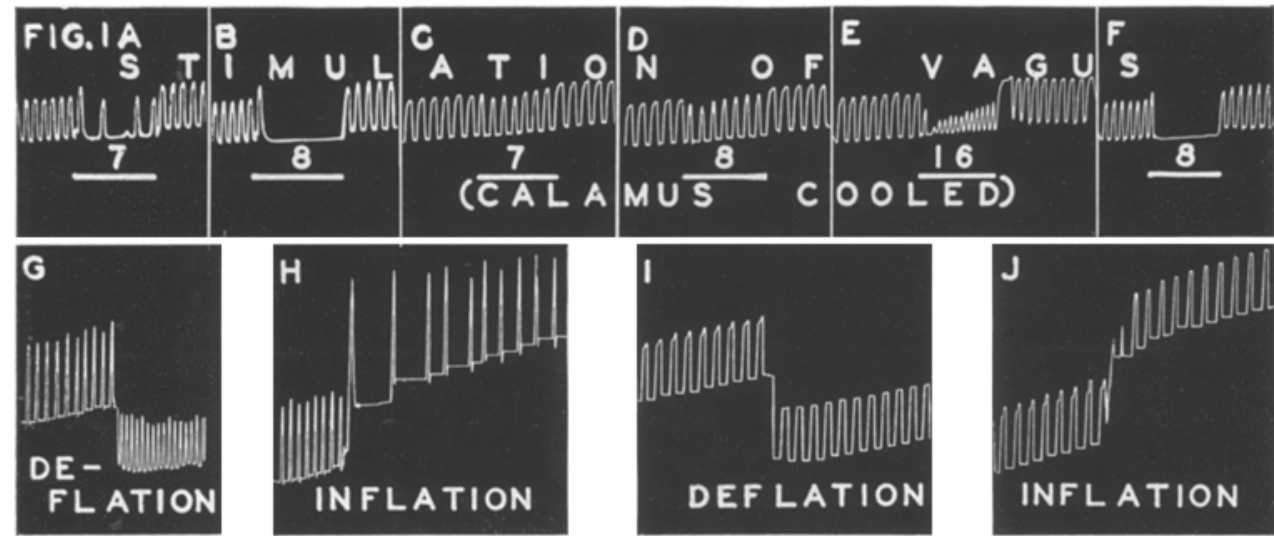

Fig. $103 \mathrm{~A}$ to J. Cooling of the medulla abolishes the retardation of breathing produced by faradic stimulation of the central end of the vagus nerve. After cooling stimulation produces acceleration. Compare $\mathrm{A}, \mathrm{B}$ and $\mathrm{F}$ with $\mathrm{C}, \mathrm{D}$ and $\mathrm{E}$. The slowing action of pulmonary inflation in record $\mathbf{H}$ are missing after cooling of the medulla (see records I and J). [NICHOLSON and BREzIN: A mer. J. Physiol. 118, 441 (1937).]
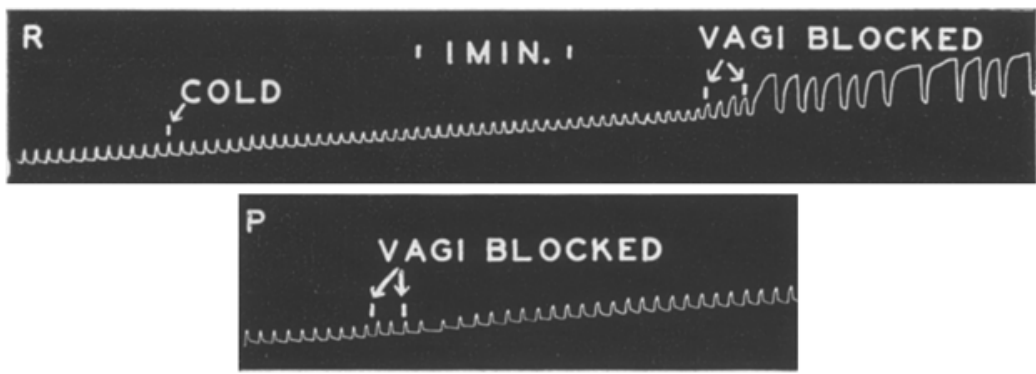

Fig. $103 \mathrm{P}$ and $\mathrm{R}$. Vagal block alone may produce little effect on breathing, $103 \mathrm{P}$. Slight cooling of the medulla may produce little change in breathing, $103 \mathrm{R}$. But the combination of medullary cooling and vagal block may prodnce striking apneustio breathing. [NIOHOLson and BREzIN: Amer. J. Physiol. 118, 441 (1937).]

(1931) as a result of pontile transection, by Henderson and SwEET (1929) as a result of section of the rubrospinal tracts plus section of the vagi, and by TAYLor $(1930,1931)$ as a result of gradual poisoning by hydrocyanic acid gas. We have observed it as a result of severe cooling of the obex or slight cooling plus blocking of the vagi. All of these procedures might conceivably reduce the frequency of impulses arriving at some internuncial neuron in the inspiratory pathway. It seems quite likely that the central respiratory mechanism receives impulses from various higher centers via the descending tracts of the brain stem as well as from many peripheral receptors via various afferent nerves such as the vagus and trigeminal. There is of course known to be an 
increase in impulse frequency in the vagi during normal inspiration. It does not seem necessary to assume, as has LuMsden, the existence of a special center in the upper pons with the specific function of periodically interrupting inspiration in order to explain apneusis as a result of pontile transection." It will be seen that this explanation is in good accord with the original views of Marckwaid.

As Marckwald found, Stewca also finds that brain stem transection plus vagotomy are the essentials for apneusis. Cervieal and cranial nerves play no important role. She believes that her "observations fit very well with the old view of Marckwald 1890 who regarded apneusis as the expression of the unrestrained activity of the inspiratory center and regular respiration as

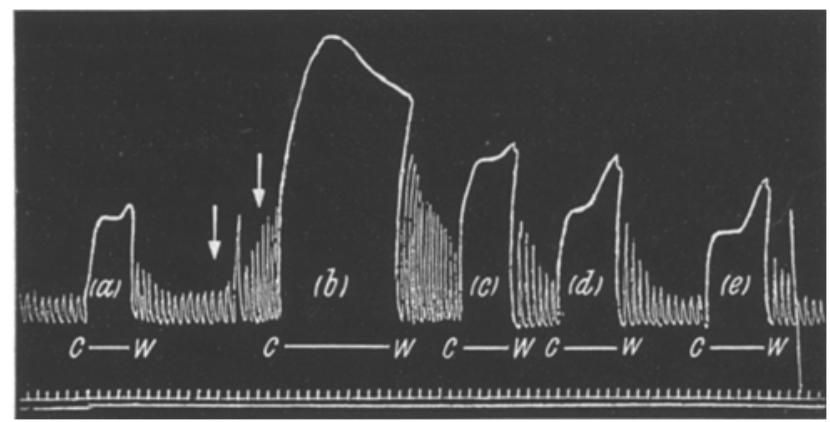

Fig. 104. Vagal block after brain stem transection during varying degrees of hypercapnia. The greater the hypercapnia the more intense is the apneusis. Upstroke indicates inspiration. ISTELLA: J. of Physiol. 93, $263(1938) \cdot]$

rhythmically interrupted apneusis." Like MARCKwALD she finds that the effect of transection of the brain with vagi intact is not so great as those produced by vagotomy alone and, therefore concludes that the pneumotaxic center cannot have the paramount importance in the regulation of respiration attributed to it by LuMSDEN.

STELLA finds that the administration of $\mathrm{CO}_{2}$ increases the intensity of apneusis. Her procedure is to block the vagi during different grades of hypercapnic hyperpnea. As fig. 104 shows, rapid deep breathing is transformed into inspiratory tetanus, the intensity of which varies with the degree of hypercapnia. When the experiment was done in a reverse order and carbon dioxide administered during apneusis both frequency and depth of breathing are increased. This is seen in LuMsDens experiments (fig. 105) and also in the experiments of Nicholson and Brezin. The latter state that the chilled center is highly sensitive to the accelerating action of $\mathrm{CO}_{2}$ which is supported by their fig. $106 \mathrm{~A}, \mathrm{~B}$ and G. MacDowar (124) describes the same accelerating action of carbon dioxide on apneustic breathing.

It is interesting to compare the opposite effects of sodium carbonate during eupnea and apneusis. During eupnea it produces an expiratory apnea 


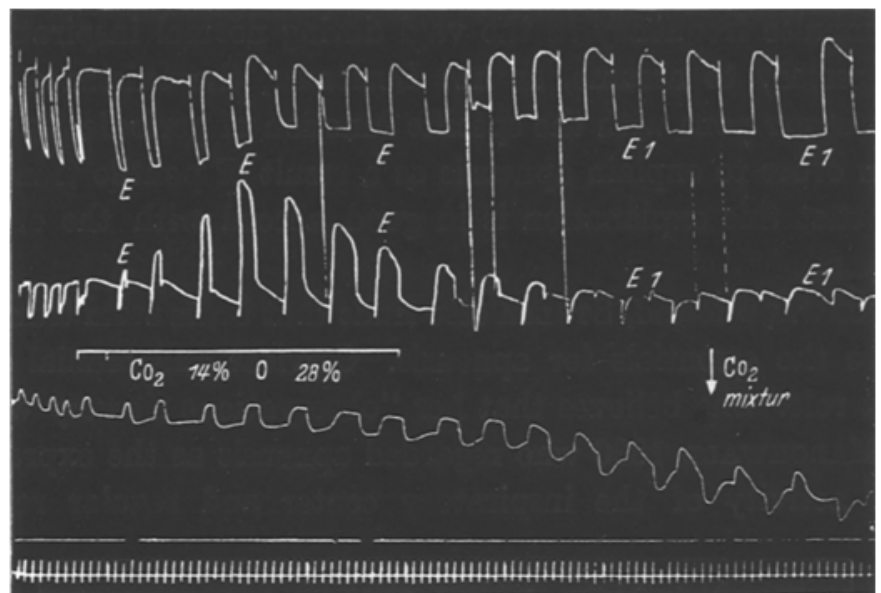

Fig. 105. Carbon dioxide administered during apneusis does not abolish rhythmie breathing as it did in STELLAs experiments. In fact the tidal air excursions are increased. The inspiratory spasms are however prolonged. E indicates expiration. [LuMsden: J. of Physiol. 57, 354 (1922).]
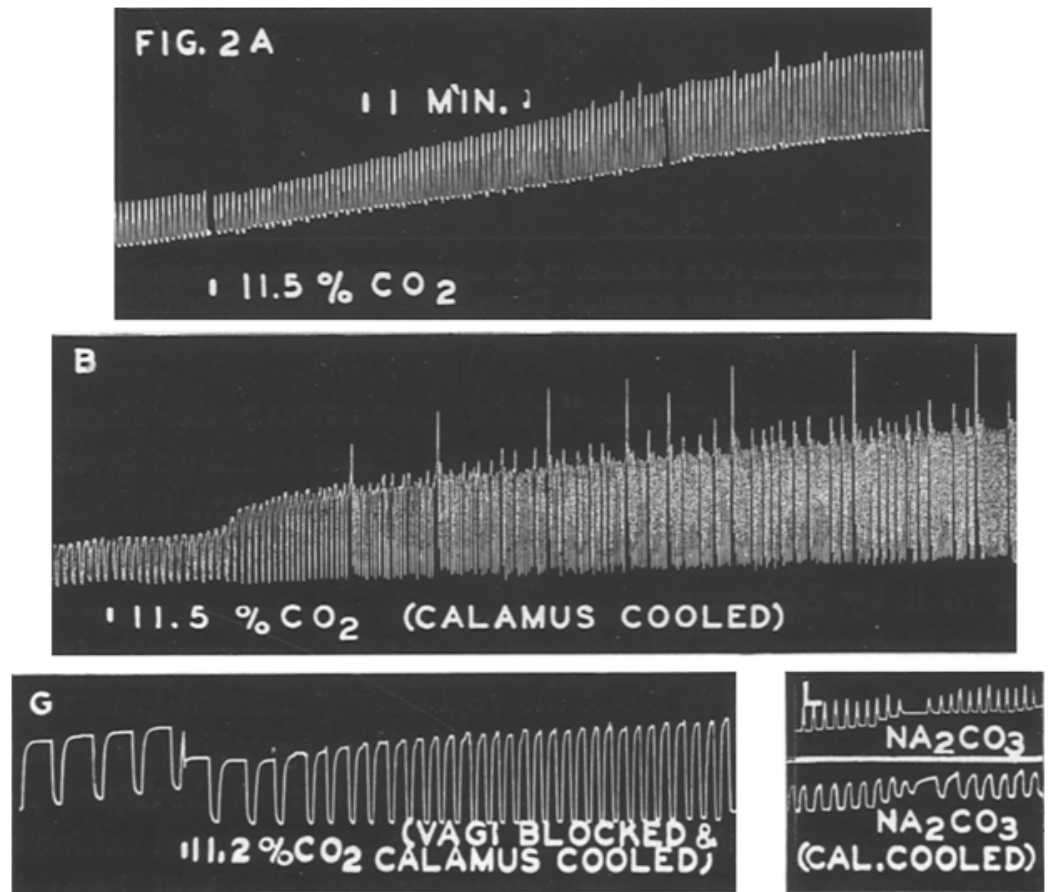

Fig. 106. Carbon dioxide produced only a small acceleration of breathing in the intact dog. Record A. After cooling of the calamus, and record B, after combined cooling of the calamus and vagal block the accelerating action of carbon dioxide was much greater. The small figures in the lower right hand corner show the opposite effects of $\mathrm{Na}_{2} \mathrm{CO}_{3}$ under normal conditions and during apneustic breathing. For explanation see text. [Nicholson and Brezin: Amer. J. Physiol. 118, 441 (1937).]

or spasm, during apneusis it produces inspiratory apnea or rather intensifies and prolongs the inspiratory spasm (see fig. 106 G). Knowing that carbonate accentuates the prevailing reflexes it may be assumed that normally 
the expiratory reflexes such as those arising in the vagal proprioceptives are relatively prominent. During apneusis it may be assumed that the expiratory reflexogenic component is weak and that the inspiratory component is relatively strong due to the withdrawal of the vagal expiratory component. This is of course in line with the general interpretation of apneusis-namely a failure to interrupt the inspiratory activity by opposing expiratory activity.

More recently Pitss, Magoun and Ranson have restudied the subject of apneusis and obtained results much of which agrees in general with that of Marckwald, Lumsden, Nicholson, Stella and others in that brainstem transection and vagal section produce apneustic breathing, that the vagi and upper tracts are interchangeable in their interrupting action of apneusis, that of the two, the vagi are the more important, that stimulation of the vagus nerve or of the substance of the medulla, whether continuous or rhythmical is capable of interrupting the apneusis. There can then no longer be doubt of the existence of two influences capable of interrupting inspiration. The fact that occlusion of blood vessels [Asher and Lüscher (125)], transections and cold are capable of producing similar effects is a satisfying point. Only on details of anatomy and theory of control are there still differences of opinion and some of these we believe are based upon uncontrolled experimental data and the employment of uncertain physiological principles.

On the basis of the supposedly high selectivity of their electrodes, Pitrs, Magoun and Ranson believe that their localized central excitations are confined within a volume of $3 \mathrm{cmm}$. or approximately one-tenth of the total volume of the respiratory center. They arrive at the following deductions. They believe that "the stimulus originating in a few inspiratory neurons tends to spread throughout the inspiratory center through the rich synaptic connections of its constituent cells (gradual build up of apneusis). It spreads likewise to the expiratory center where it causes inhibition of an expiratory activity. Then through various reentering paths it tends to establish itself as a permanent circus (maintenance of apneusis). Since the inspiratory center can be excited as a whole from an electrode placed anywhere within it, multiple intercellular connections must be present. This circus can scarcely include all the inspiratory neurons for weak or moderate stimulation of the inspiratory center can bring into activity new units (facilitation) which once in activity may remain in circus after cessation of the stimulus (increased inspiration maintained as increased apneusis after cessation of stimulation). Stimulation of the expiratory center directly or indirectly through its connection with vagal afferents leads, as we have suggested previously, to inhibition of inspiration through raising of synaptic resistance between neurons of the inspiratory circus (breaking the circus) as well as bringing about in some cases active expiratory activity. Such increase in synaptic resistance within the inspiratory center may merely be effective in eliminating some neurons from the circus 
(reduction in apneusis) or may abolish the circus (complete expiratory relaxation or active expiration). A strong stimulus, however, to the inspiratory center tends to set into activity simultaneously many neurons of the center producing maximal inspiration (replacing the circus with synchronous activity of many neurons). The depression of the excitability in many neurons, following their synchronous activity, at the cessation of the stimulus interrupts and prevents reestablishment of circus immediately (complete inspiratory relaxation on cessation of a strong inspiratory stimulus). As recovery progresses the circus reestablishes itself (building up apneusis again)."

These statements may be considered from several points of view, the first of which has already been discussed, namely the validity of the method. If BRookharts findings on his own high voltage experiments are transferable to those of Putss, Magoun and Ranson, they will have need to modify their structure of reasoning or show by some direct experimental procedure that their method is dependable. Second, the use of the circus impulse as a substructure of rhythmic activity while attractive does not seem to be demanded. Third, in the light of hundreds of experiments done at the Michigan Laboratories the use of a synchronisation mechanism in nervous integration seems very precarious. It is true that Adrian and Bronk (5) and RiJuant (8) stress the use of this phenomenon as a nervous tool but we have been unable to confirm their results in the dog, rabbit, rat or mouse. Wyss (107) has met with the same difficulties. Motor integration, and probably nervous integration in general, is an asynchronous and not a temporal process. The strong expiratory contraction following powerful inspiratory stimulation, seen in fig. $107 \mathrm{C}$ at $4,6,8$ and 12 volts is attributed by Pitrs, Magoun and RAsson to fatigue of the inspiratory center interrupting the inspiratory circus. While we agree that the sudden and powerful inspirations can come to an end as a result of fatigue, we believe this is normally dependent on the interrupting action of accelerated firing described under the recurrent collateral, vagal and Gougr reflexes. But we suggest that the powerful expiratory contractions noted by Prtrs, Magoun and Ranson be considered as rebound (refer back to fig. 86 and 87 and discussion). It will be recalled that a sudden decrease of activity of an inspiratory cell e. g. \# 1 in fig. 58 resulting from cessation of a powerful electrical stimulation should excite expiratory cell \# 1 by withdrawing its restraining negativity which it has been impressing on the expiratory axon hillock. As a result, expiratory cell $\# 1$ becomes momentarily active and impresses negativity on the axon hillock of inspiratory cell \#1 and so suppresses inspiratory activity. The inspiratory muscles, therefore, relax. This phenomenon of rebound seems to be relatively common and often occurs with spontaneous superposition of sharp inspirations on apneustic contractions. See fig. 108 from Putts, Maqoun and Ranson and fig. 109 from Lumsden. The conclusion of Pitts, Magoun and Ranson 
on the origin of rhythmicity is most difficult to follow. They state: "The question as to whether rhythmic respiration results from automatic processes inherent in the respiratory center or not, resolves itself in two parts. Is activity within the center spontaneous; and is that activity spontaneously
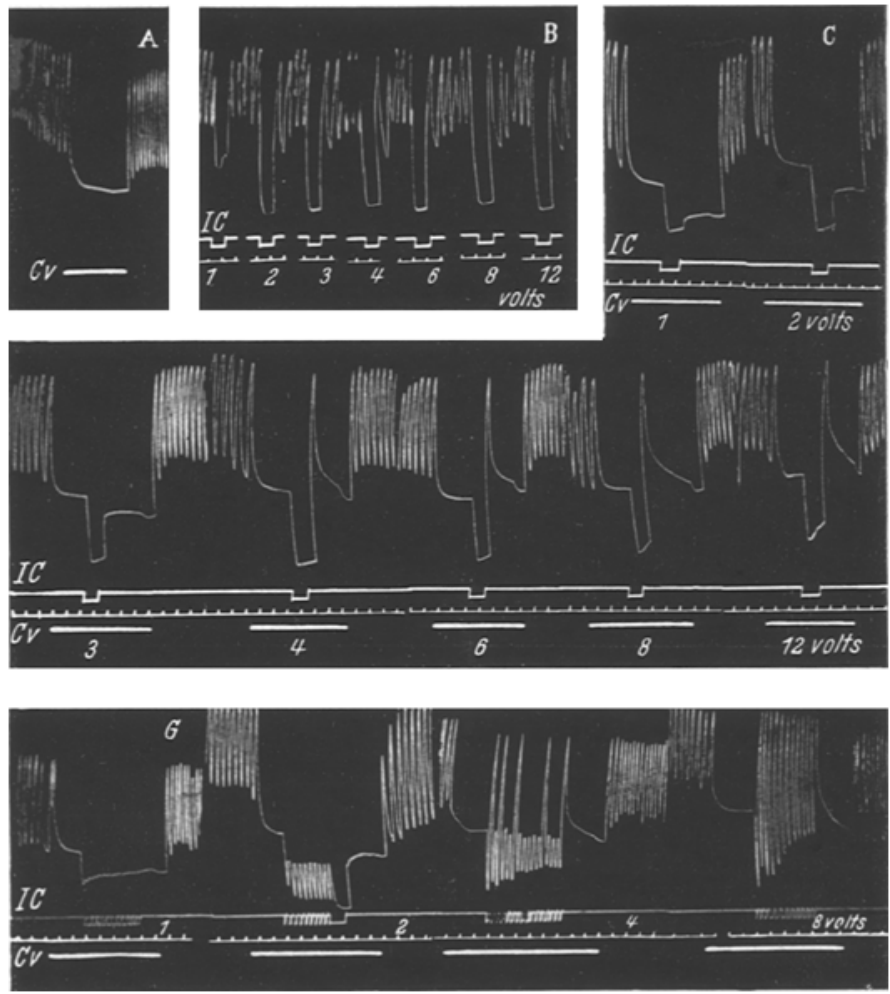

Fig. 107. Respiratory reaction from stimulation of the inspiratory and expiratory centers in pontile decerebrate animals during apneusis produced by cold blocking of the vagi. Cv, cold block applied to vagus. A, typical apneusis on cold blocking of the vagus. $B$, inspiratory reactions to stimulation of the inspiratory center with voltages indicated. C, same voltages during apneusis produced by cold blocking the vagi. Note that from 1 to 3 volts the apneusis is maintained on cessation of the stimulus while from 4 to 12 volts it is temporarily released. G. apneusis converted into rhythmic respiration by stimulation of the inspiratory center. [PITTS, Magoun and RaNson: Amer. J. Physiol. 126, 689 (1939).] We omitted records $\mathrm{D}, \mathrm{E}$ and $\mathrm{F}$ because the figure is used primarily to illustrate the release of apneusis by superimaposed inspiratory aetivity. Compare the effects of periodic superimposement of inspiratory activity in record $G$

( 8 volts) with the effects of periodic stimulation of the vagus nerve in fig. 86 explained as rebound.

rhythmical? From the work of Marchwald, Stelua, Pitts, Magoun and RANSON and the experiments reported above, the latter question must be answered in the negative. Rhythmicity is imposed on the medullary respiratory center by two bilateral mechanisms: the one a vagal pneumotaxic mechanism; the other, a brain stem pneumotaxic mechanism located in the upper few millimeters of the pons." And finally they conclude that "Spontaneous rhythmicity is not a property of the medullary center." This statement is made although they themselves, like many other workers, have registered rhythmic respiratory movement after vagotomy and brain transection. In Marckwalds 
own conclusion, published in his studies of inspiratory tetanus, the following statement occurs.
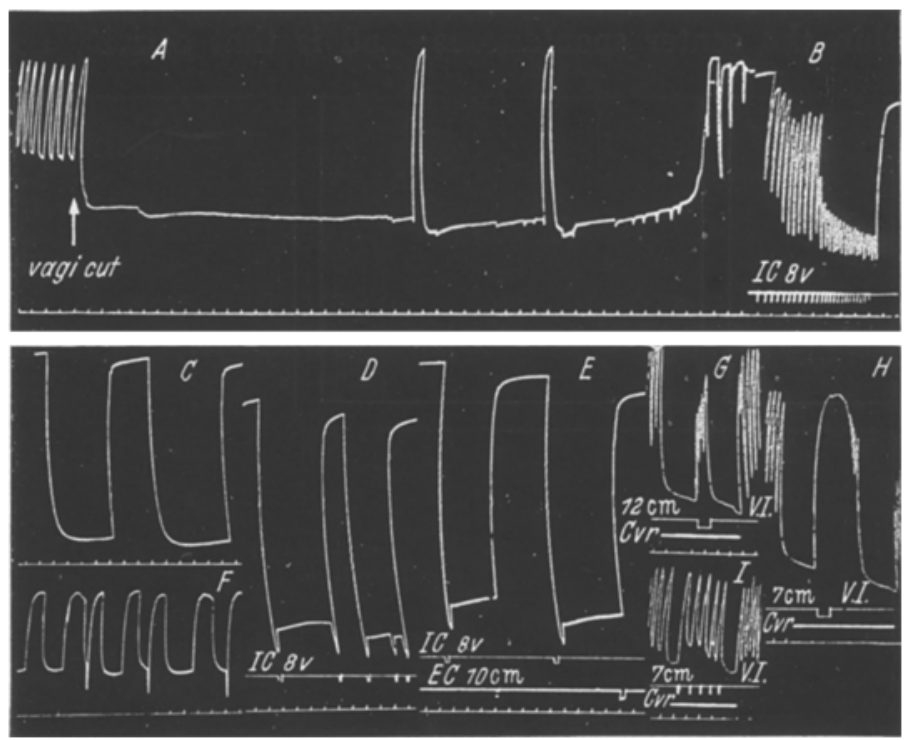

Fig. 108. Respiratory reactions from stimulation of the inspiratory and expiratory centers and the vagus in pontile decerebrate animals during apnensis produced by cutting or cold blocking the vagi. A, typical apneusis in a pontile decerebrate animal resulting from bilateral tagal sections. $B$, stimulation of the inspiratory center after cessation of respiration in A. $\mathrm{C}$, spontaneous apneustic respiration after prolonged artificial ventilation. D, stimulation of the inspiratory center in animal C. E, stimulation of the inspiratory and expiratory centers in 2 similarly prepared animal. F, spontaneous apnenstic respiration with apneusis released by gasps. G, H, I stimulation of the central end of the sectioned left vagus during cold blocking of the right vagus. [PITTS, MAGOUN and RANson: Amer. J. Physiol. 126, 689 (1939).] This figure is of special interest in showing spontaneous as well as artificial superimposition described in the text of inspiratory activity. In record $A$ apneusis is interupted at three points by tiny clonic inspiratory efforts. As each inspiratory tetanus exhausts itself it would seem to become more susceptible to interruption through the process of rebound (see fig. 110 and 111). Record $D$ shows rebound interruption of apneusis with electrical stimulation and $\mathbf{F}$ with spontaneous gasps.

"I, therefore, come to the conclusion that the respiratory center in the fourth ventricle can be automatically active, but the automatic mechanism manifests itself only in inspiratory and expiratory spasms."

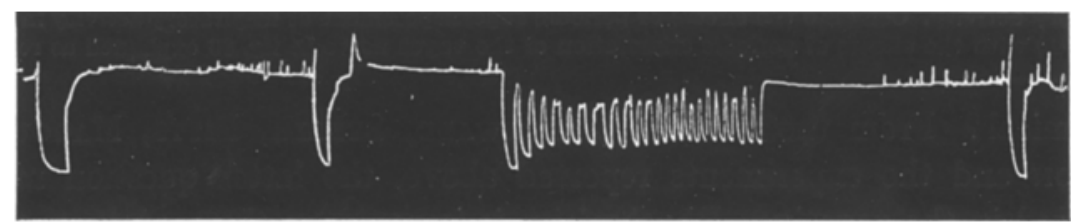

Fig. 109. Superimposition of inspiratory activity on apnensis leads to expiratory interruption of apneusis as in fig. 107 and 108. Upstroke inspiration. Also note rhythmic breathing produced by periodic stimulation of the vagus nerve each time inspiration has been taken. [LUMSDEN: J. of Physiol. 57, 354 (1922).]

How then may our views be applied to an explanation of apneustic breathing? We have already suggested the role of the recurrent collateral as a combination excitor and exhaustor of the nerve cell (see fig. 31, 39 
and 40). Theoretical as this mechanism of interruption is there are two reflexes operating in a comparable way which are on a more tangible footing-the augmenting action of lung inflation and of muscle and tendon stretch. By virtue of the progressive intensification of these reflexes the inspiratory cells are stimulated to a higher pitch of activity as inspiration progresses. Now there are two interesting experiments that bear on the implications of this point. One by Fueisch (99) and the other by Sherrington (77). In referring to the diagram of Fleisch (fig. 110), it will be seen that the inspiratory activating proprioceptive reflexes weaken as inspiration progresses while the inspiratory inhibitory reflexes strengthen with the advance of this phase. In other words the inspiratory half center becomes particularly susceptible to inhibition in the exhausted condition. SHERRINGTON puts it this way.

"Central fatigue favours reflex inhibition. Thus, a given inhibitory stimulus pitted against a given contraction background will act more powerfully i.e., will arrest more active units- when employed late in the course of the tetanic plateau than when employed early."

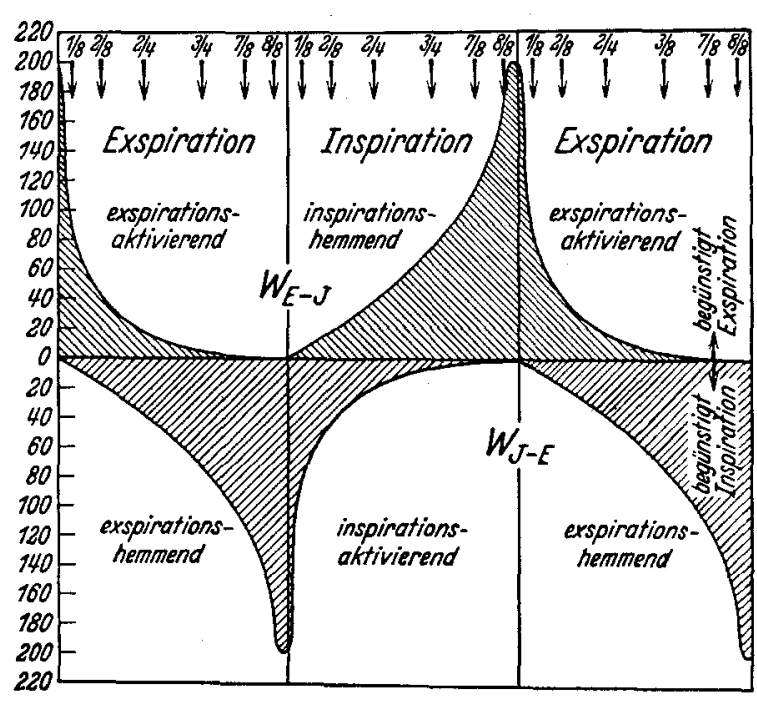

Fig. 110. Diagrammatic representation of the changing intensity of expiratory activiting and expiratory inhibiting proprioceptive reflexes during the expiratory act and of the inspiratory inhibiting and inspiratory activiating proprioceptive reflexes during the inspiratory act. $W_{E}-I$ phase change from expiration to inspiration. $W_{I}-E$ phase change from inspiration to expiration. [Fleisch: Erg. Physiol. 36, 249 (1934).]

(See fig. 111 which reads from right to left.)

This general schema would seem to fit the double vagal action which we have proposed (section 20). During inspiration the stretch receptors of the vagus nerve dispatch great volumes of impulses to both the inspiratory and expiratory half centers. However only those signals impinging on the inspiratory half center are capable of stimulation, because the expiratory center is reciprocally inhibited by the activity of the inspiratory center. The signals coming from the stretch receptors whip the inspiratory half center to increased activity, exhaust it, and prepare it for the inhibition which is soon to come from the expiratory half center. The slightest weakening of the inspiratory activity now means lessened restraint upon the expiratory half center and the initiation of expiratory activity. The inspiratory center being highly susceptible to inhibition at this moment ceases firing and gives way entirely to the inhibitory action of the expiratory half center. That tentatively is a schema for the shifting of the respiratory phases. 
Concerning the existence of respiratory centers other than the primary center in the medulla oblongata, we agree with the views of Marckwald expressed many years ago. "My experiments regarding direct stimulation of the respiratory center in the fourth ventricle have further shown that the respiratory center can only be stimulated in the same way as if the stimuli were transmitted by centripetal nerves. The upper tracts also act on the vagus center like sensory nerves, and as they have no direct connection with the same they probably act through the agency of other nerve centers which are related anatomically to the vagus center. But if one were to call every nervous distribution from which respiratory movements can be stimulated a

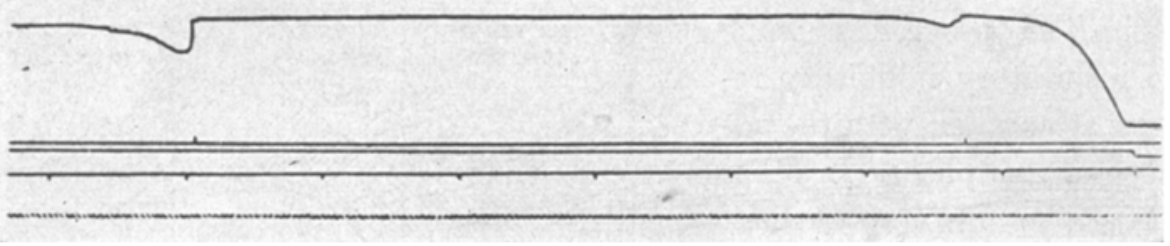

Fig. 111. Fatigue favours inhibition. Decerebrate vastocrureus; deafferented; reflexly activated by contralateral plantar nerve at 30 double shocks per sec. coil at $19 \mathrm{~cm}$. Inhibition by single-break current to ipsilateral plantar nerve at $16 \mathrm{~cm}$. of coil. Five seconds interval between the two inhibitory reflexes. Tension of plateau, 1,8 kil. (D. Denny-Brown: Selected Writings of Sir Chartats Sherrivgton, PaUL B. Hoeber. New York: Inc. Medical Book Departement of Harper and Brothers 1940.)

respiratory center, then the discovery of such new centers would have little value to science and would bring little honour to the discoverer. At the very least, one must demand from a centre, that in it centripetal tracts terminate and centrifugal tracts arise, and that its disappearance destroys or materially disturbs any function which it governs." It is on this basis that MARCKwaLD concluded that there are no respiratory centers above the primary center in the medulla for transections above that important area of convergence have revealed only the slightest effects upon breathing. Certainly there is no justification for placing a center either in the lungs or at the endings of the superior laryngeal nerve yet these structures exert a more powerful interrupting action on inspiration than do the descending brain impulses. Until more comprehensive data are accumulated it would seem desirable to think of the descending brain stem impulses as we think of influences ascending from the vagal stretch receptors.

\section{The Role of the Spinal Cord.}

Very little is known about the role of the spinal cord in the shaping of the respiratory act. Though regarded as little more than a substation for the relay of signals from the primary center to the respiratory muscles it will no doubt prove to have decidedly broader functions. The intimate connections of the anterior horn cells with the incoming sensory signals speak for that. But more important are the well established facts on the modification of 
spinal reflexes, by superimposition of proprioceptive influences after a reflex is started on its way [Creed, Denny Brown, Eccles, Liddell and

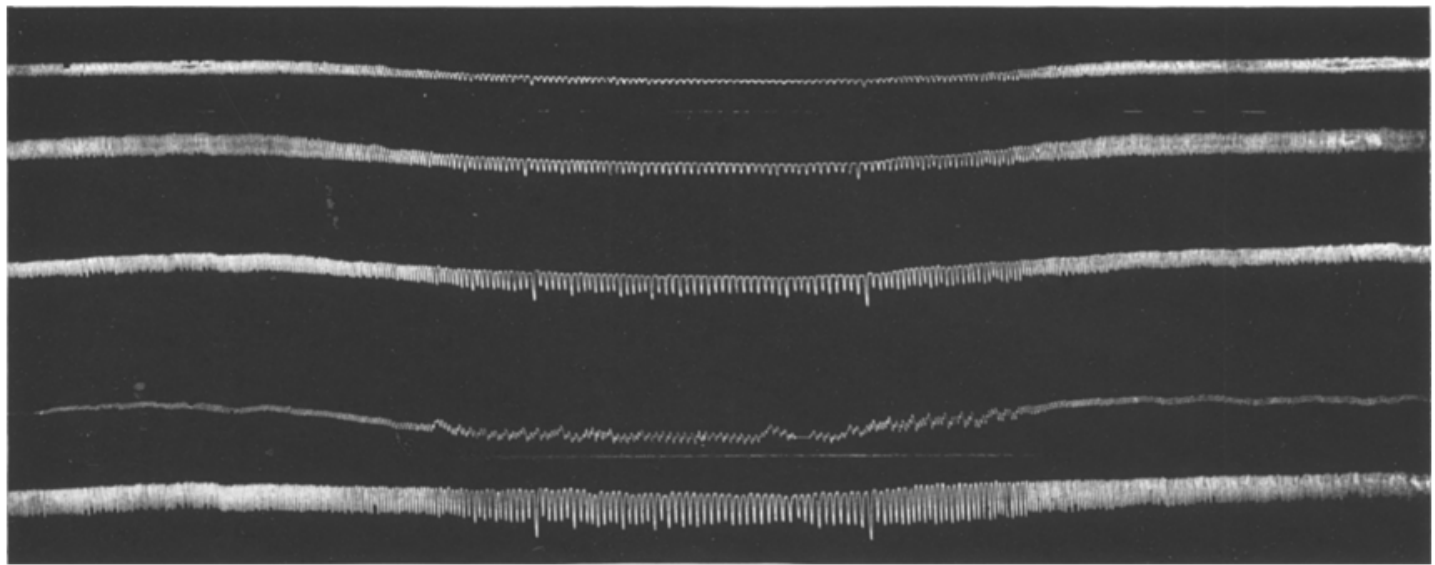

Fig. 112. See text. [Geselul and Moyer: Quart. J. exper. Physiol. 25, 13 (1935).]

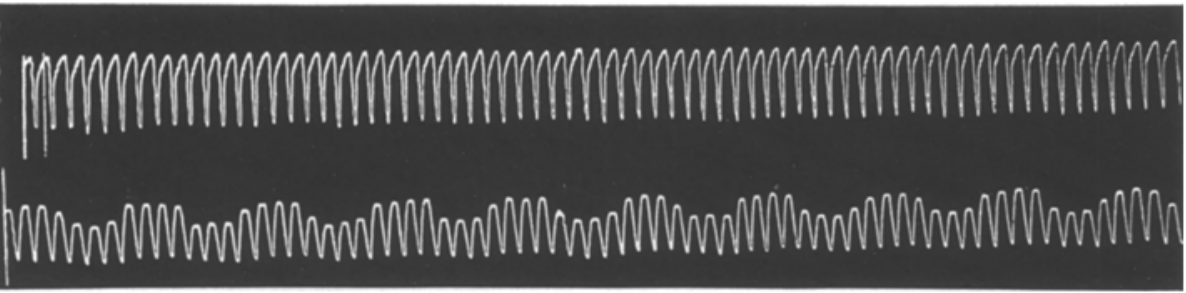

Fig. 113. See text. [Gesell and Moyer: Quart. J. exper. Physiol. 25, 13 (1935).]

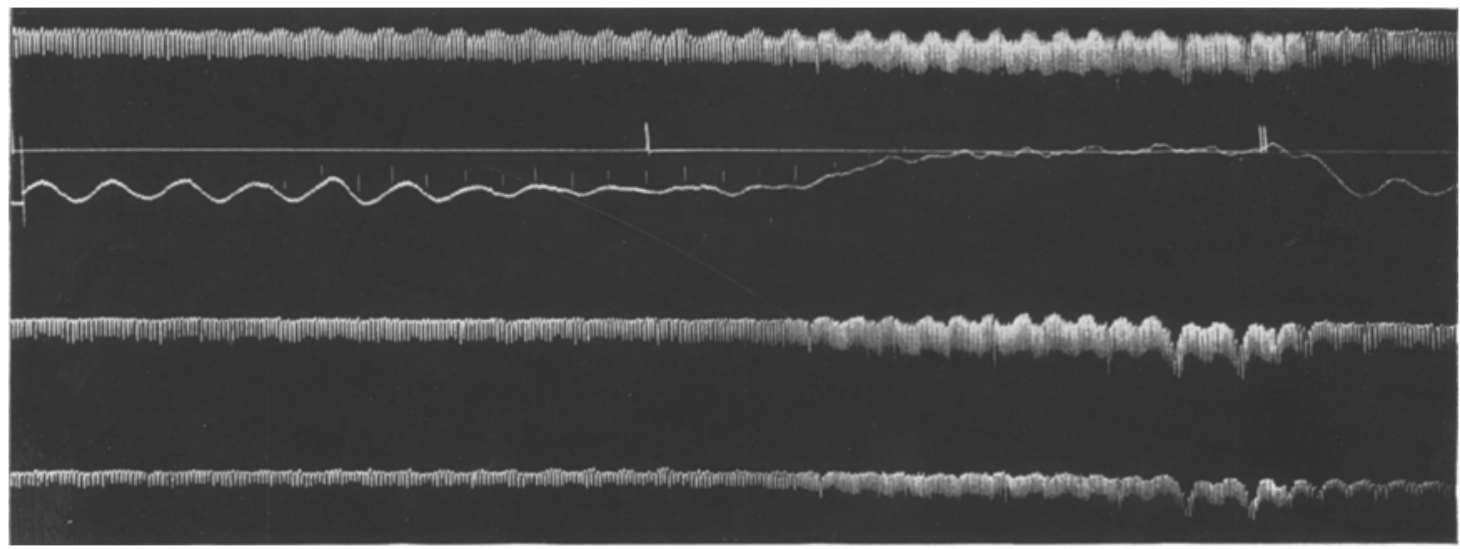

Fig. 114. See text. [Gesell and Moyer: Quart. J, exper. Physiol. 25, 13 (1935).]

Sherrivaton (59)]. We need only picture a superposition of spinal reflexes upon the respiratory activity pattern dispatched from above to realize that much is still to be revealed in this phase of respiration. An example of reinforcement 

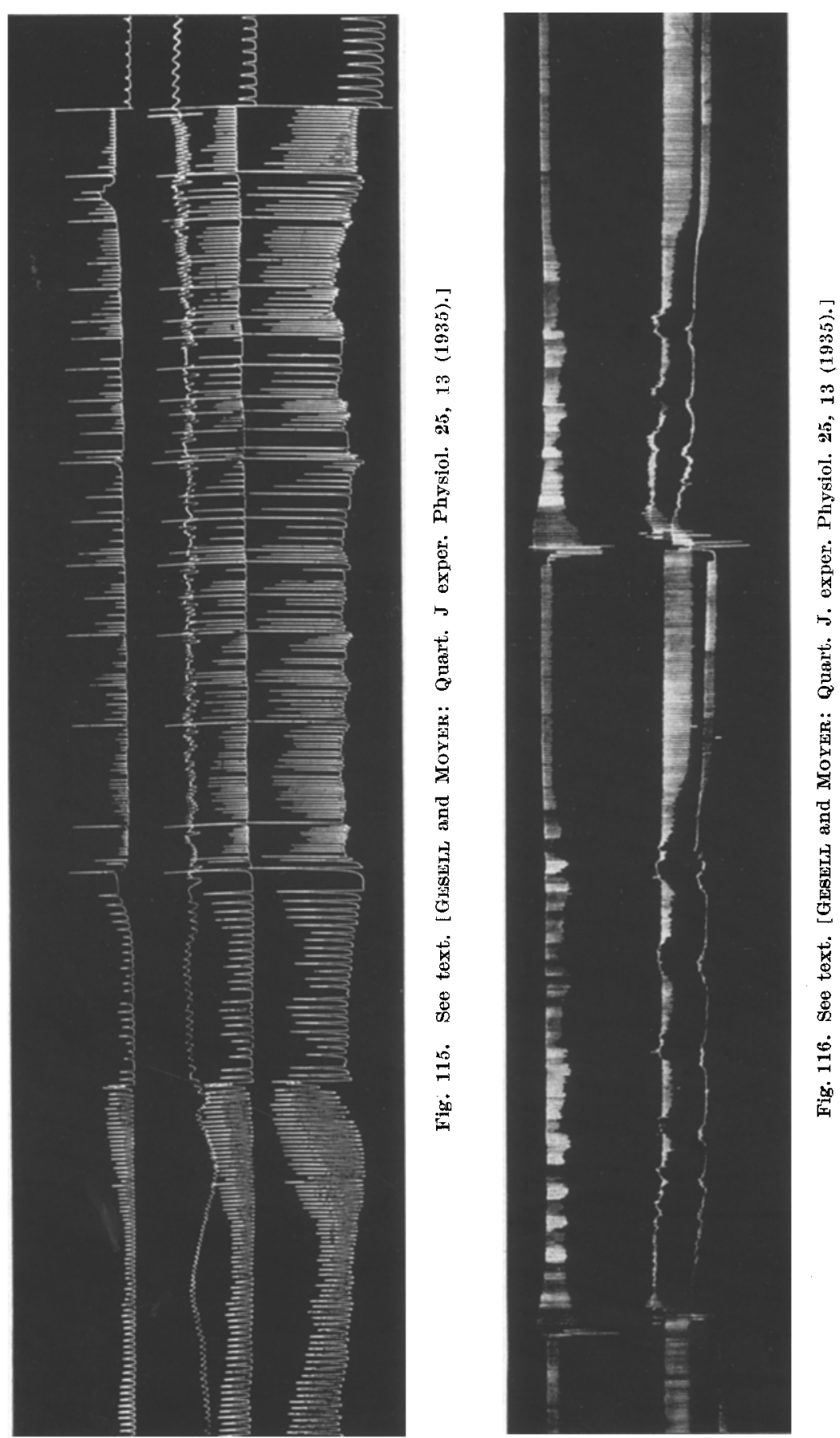

of the inspiratory act through the superimposement of the GoLGI reflex, set up by inspiratory obstruction has already been mentioned. To what extent this particular adjustment is brought about through local spinal reflexes 
and through the longer circuit reflex involving the cuneate and gracile and reticular nuclei remains for future study.

The startling respiratory phenomena encountered by continuous registration of costal and abdominal respiratory movements suggests most striking divergences of costal and abdominal activity are possibly attributable to the cord [Geselu and Moyer (126, 127)]. Fig. 112 to 118 will illustrate.

The upper record or records are registered by bands encircling the chest. The lower records are from the abdomen. Sometimes prolonged periodic

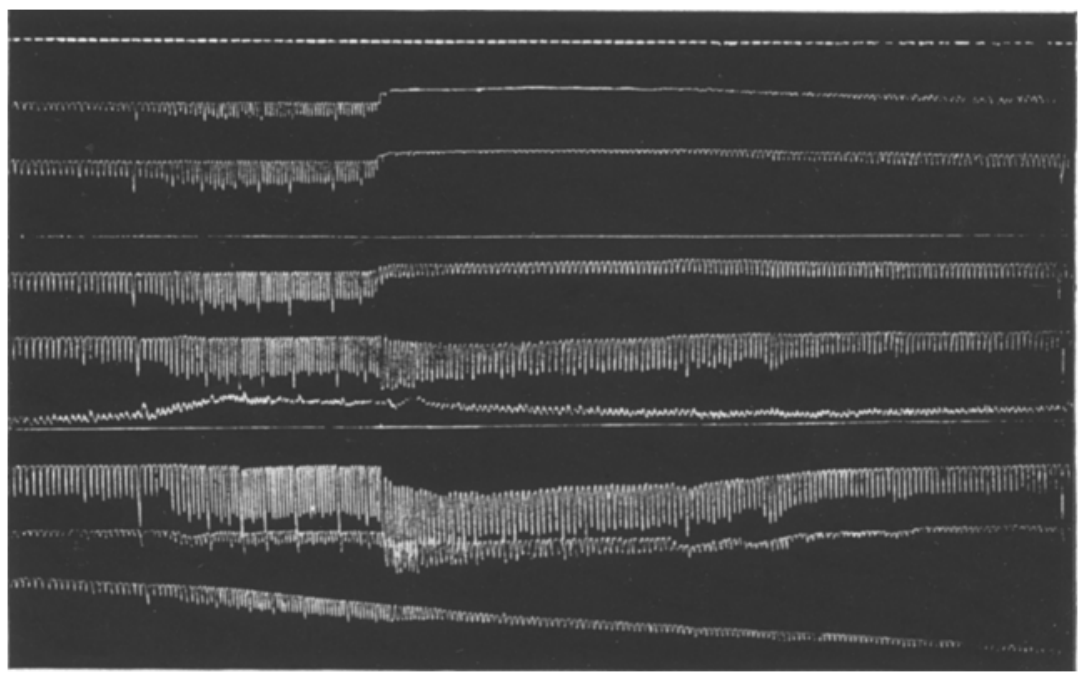

Fig. 117. Effects of oxygen deficiency and readministration of room air on costal and abdominal respiratory excursions. Records 1,2 and 3 are from the chest. Records 4, 5 and 6 are from the abdomen. $\mathrm{S}$ is a spirometer record of tidal air. Note the uniform increase of all excursions during oxygen deficiency and contrast with the complete suppression of costal excursions and actual increase of abdominal excursions with readministration of room air. The spirometer on the other hand shows a uniform expected recovery. [Gesell and Moyer: Quart. J. exper. Physiol. 24, 331 (1935).]

changes of unknown origin would occur, as in fig. 112, in which the costal excursions diminished and the abdominal strokes increased. Sometimes periodic cycles of short duration appeared in the abdominal record while the costal record remained astoundingly uniform, as in fig. 113. But periodicity might appear, first in the costal record only to show up later in the abdominal tracing, as in fig. 114. Or irregularity of the expiratory position might be limited to the chest alone, as in fig. 115. In occasional experiments it was not uncommon for our dogs to stretch their extremities. When that occurred the proportion of chest and abdominal excursions might change in the manner shown in fig. 116.

The effects of chemical changes produced by oxygen lack and by carbon dioxide excess also open new fields of study. Note for example the action of oxygen lack in fig. 117. Oxygen deficiency usually produces a tendency towards a residual inspiratory tonus at the end of expiration, at all levels 
of the torso. In this particular animal the phenomenon is missing, but another common and most striking result occurs on readministration of room air. The chest excursions decrease far below normal (they actually disappear in the upper record) while the abdominal excursions actually increase in amplitude above that of the immediately preceding hyperpnea. Had only the

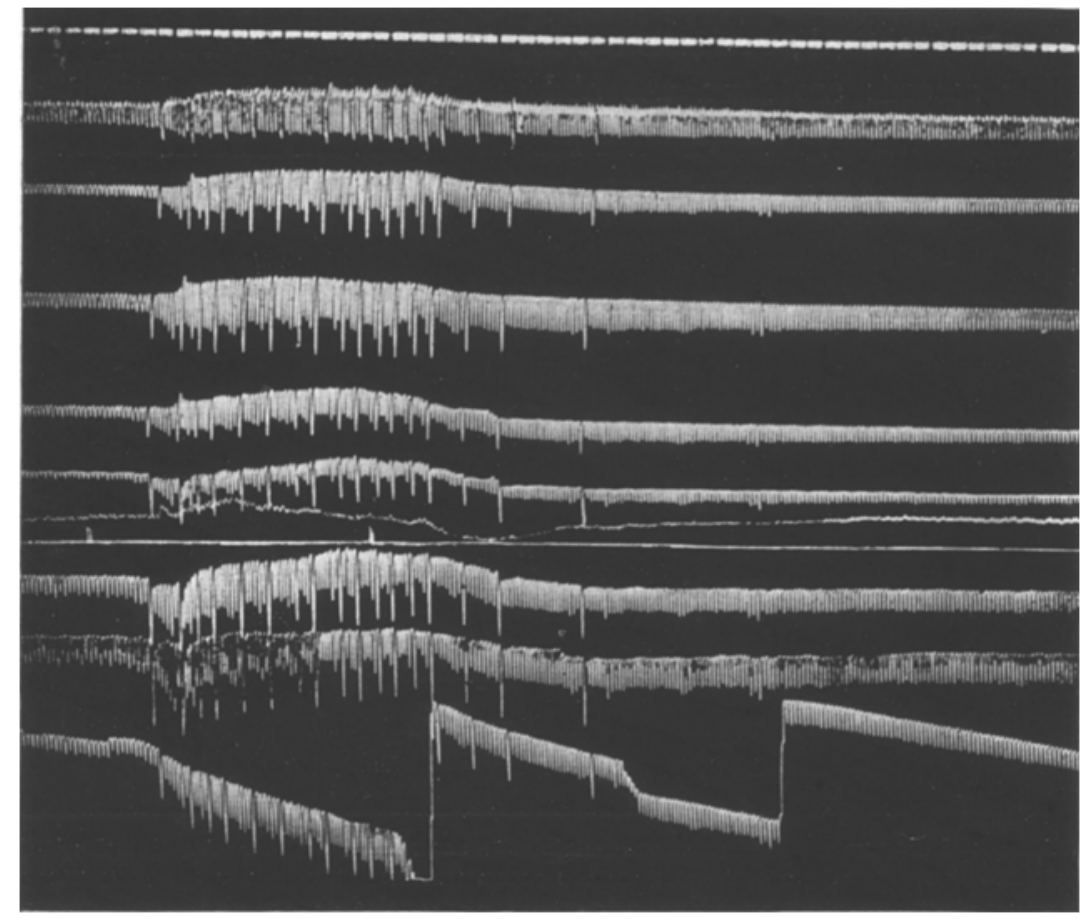

Fig. 118. A similar set of tracings as those obtained in fig. 117, obtained during the administration of carbon dioxide. The effects are similar at all segments during hypercapnia and recovery. Note the increased expiratory activity at all levels. [GEseLI and MoxeR: Quart. J. exper. Physiol. 24, 331 (1935).]

tidal air changes been recorded this strange coordination of costal and abdominal activity would have been overlooked for the tidal air recovery is that which is usually obtained.

Hypercapnia tends to produce a general constriction of both the chest and abdomen at the end of expiration such as is illustrated in fig. 118 and differential effects upon the chest and abdomen are usually missing.

\section{The Harmony of Physical and Chemical Drives.}

One of the most fascinating phases of respiratory control is the interaction of the diverse forces which drive the respiratory act. Considering the relatively uniform concentration of hydrogen ions and of oxygen molecules existing in the blood of normal individuals a most harmonious interaction between the physical and chemical drives must prevail because slight modifi- 
cations of existing proprioceptive reflexes bring chemical changes in their wake (see for example fig. $119 \mathrm{~A}$ and $\mathrm{B}$ ). In fig. $119 \mathrm{~A}$ breathing stops completely as a result of superinflation of the lungs and begins once more only after the hydrogen ion concentration of the blood has mounted and after

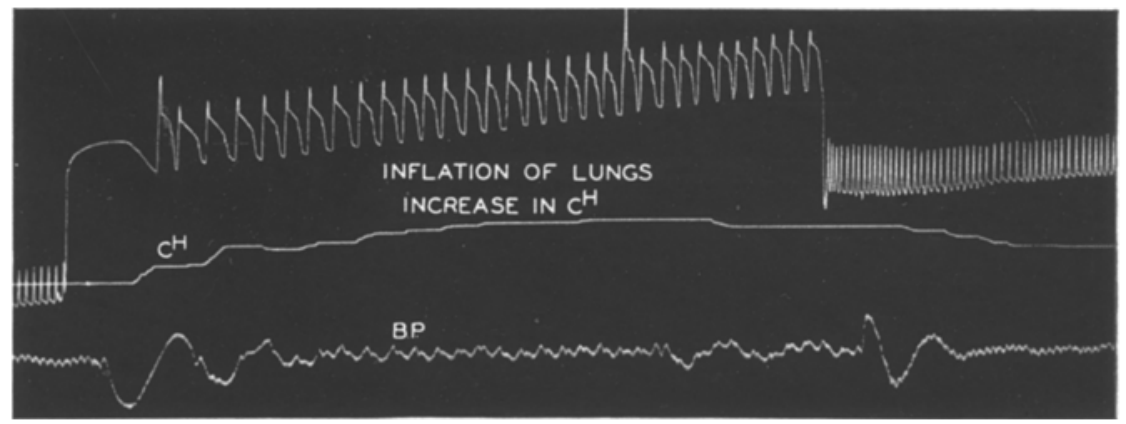

Fig. $119 \mathrm{~A}$. Effects of sustained superinflation of the lungs upon pulmonary ventilation and blood $\mathrm{cH}$, continuously recorded with the glass electrode. The inflation is produced by weighting a specially constructed spirometer (shown in fig. 64). Breathing is completely stopped until the increasing chemical stimulus counteracts the proprioceptive reflex. The chemical stimulus (excess $\mathrm{CO}_{2}$ and $\mathrm{O}_{2}$ want) remains above normal until the lungs are again unloaded.

oxygen has been depleted. Were it not for the excitatory action of these chemical changes, reflexes of purely physical origin arising in the lungs would no doubt end in death. How then is the individual rescued from these dangers?

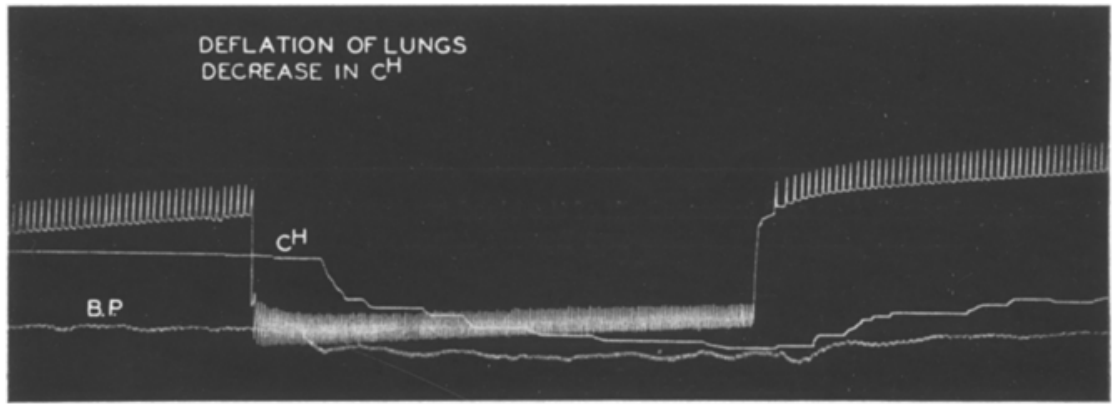

Fig. 119 B. Deflation has the opposite effects. Excessive elimination of carbon dioxide and increased oxygenation of the blood reduce the excitatory action of the proprioceptive reflexes. The blood however remains excessively alkaline. [Gesell, Lapides and Levin: Amer. J. Physiol. 130, (1940).]

As we have seen, chemical stimulation can add its effects to any form of stimulation and thereby reinforce the activity of the prevailing phase of breathing, whichever it may be. Thus the accumulation of carbon dioxide and the diminution of oxygen which occurs during a prolongation of expiratory activity produced by artificial pulmonary inflation may actually intensify the exspiratory act. Fig. 68 is a striking example of such action. Such increased activity of the expiratory half center must also intensify the reciprocal inhibition of the inspiratory half center and thus establish a vicious cycle ending in death. The rescue comes from the other side of the respiratory center. The 
predominating action of chemical stimulation on the inspiratory half center gains control and a new order of breathing is established. This breathing necessarily occurs at a higher $c_{\mathrm{B}}$ level for the stretch reflex of the lungs is reciprocally inhibiting the inspiratory half center. The reduction of the inspiratory electrotonic excitation current resulting from this inhibition can only be overcome by an increased intensity of chemical stimulation namely increased $c_{H}$ and lowered $\mathrm{O}_{2}$. These chemical changes, to be sure, are injurious to normal

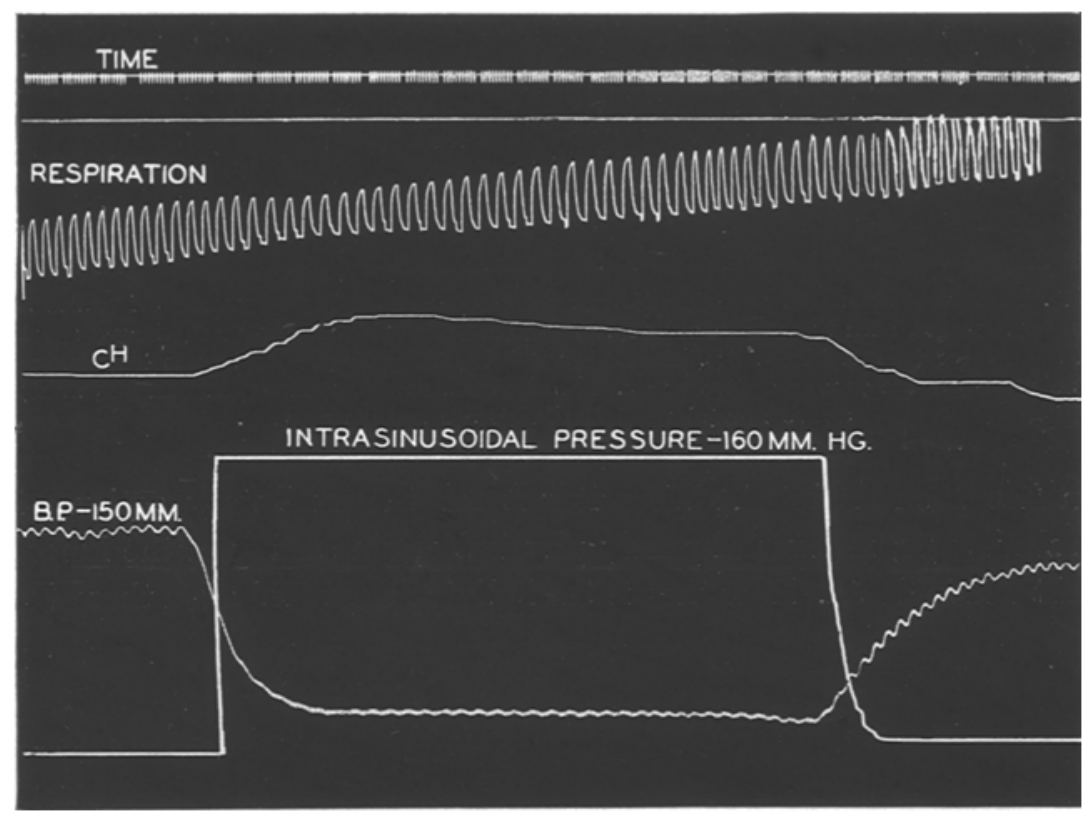

Fig. 120. Decreased ventilation and increased $\mathrm{cH}$ of the blood produced by super distention of the carotid sinuses. [Gegeid, Lapines and Leivin: Amer. J. Physiol. 130 (1940).]

oxidations nevertheless the newly established order of breathing is a decided improvement over a permanent apnea.

A steady state of deflation of the lung, readily accomplished by counterweighting the mercury spirometer reverses the situation. Now breathing becomes excessive, strikingly so at the very beginning. The $\mathrm{c}_{\mathbf{H}}$ of the blood diminishes and the oxygenation must increase. Both effects, theoretically, would oppose the increased breathing initiated by the modifications of the proprioceptive reflexes produced by deflation and as the record shows pulmonary ventilation does diminish. Again a new order of breathing is set up, this time at a lower $c_{H}$ and higher oxygen level than normal. If it is to be assumed that the normal acid base equilibrium is the optimum, the organism is again working at a disadvantage. When the spirometer is unloaded breathing is temporarily diminished indicating that hypocapnia had actually been established. HARRisor and his associates (123, 129, 130, 131 and 132) maintain 
that disturbances in proprioceptive reflexes occurring in congestive heart failure may play a significant role in the explanation of prevailing hyperpneas.

Another proprioceptive reflex contributing to the control of breathing arises in the physiological stretch and collapse of the carotid sinuses and aortic arch (see fig. 120). Despite a striking fall of blood pressure, which in

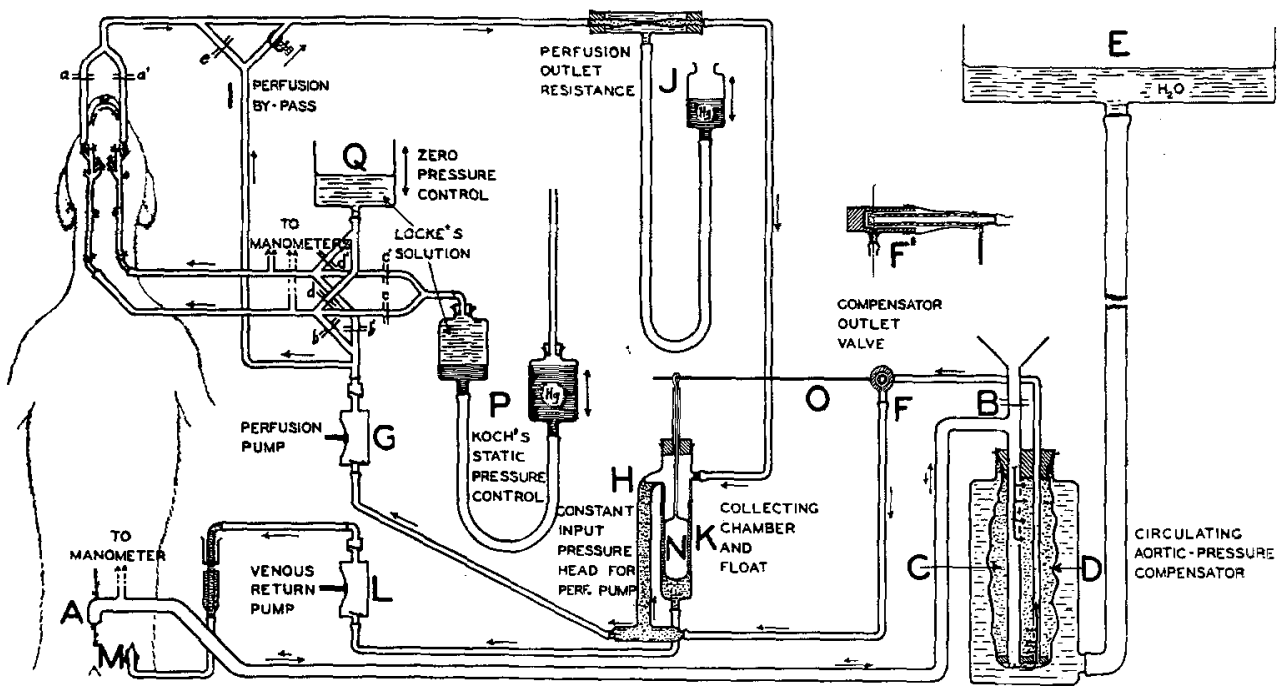

Fig. 121. Carotid segment connections and circulating aortic-pressure compensator. A to B, large-calibre, rigid connection between abdominal aorta and pressure compensator; $\mathrm{C}$, circulating blood reserve for pressure compensation, within nonresistant, collapsible separating bag $\mathrm{D}$; E, adjustable water pressurehead applied through $\mathrm{D}, \mathrm{C}, \mathrm{B}$ and $\mathrm{A}$ to abdominal aorta; F, F', automatic compensator-circulation outletvalve, supplying inlet to carotid perfusion pump $G$, with a constant, overflowing pressurehead $H$; $L$, venous return pump; M, femoral vein connection. By setting the output of venous return pump $L$ slightly greater than that of perfusion pump $G$, the float $N$ so operates compensator outlet valve $F$ through lever $O$, as to insure an overflow from pressure head $H$, and the constant presence of blood in collecting chamber $K$ for return pump L. By proper manipulation of clamps $a, a^{\prime}, b, b^{\prime}, c, c^{\prime}, d, d^{\prime}$ and e, either or both carotid perfusions can be by passed and either Kochs (1931) static pressure control, $P$, or the zero-pressure control Q, connected with either or both carotids, Abdominal aortic and each carotid pressure are recorded by respective mercury manometers. The experimental animal is heparinized. The eircuit is supplied with heparinized whole blood from a large donor dog. A constant temperature of $37,5^{\circ} \mathrm{C}$. is maintained by a circulating electrically controlled water bath and insulated or water-jacketed connections with the animal. [Cladde V. Winder: Amer. J. Physiol. 129, 306 (1938).]

itself should increase breathing, sustained artificial distention of the vascularly isolated carotid sinuses, definitely decreased pulmonary ventilation. Only as the $\mathrm{c}_{\mathrm{H}}$ increases and the oxygen diminishes does ventilation increase again. The original chemical equilibrium existing before the sinuses were inflated however is not reestablished. It would, therefore, seem that modifications of circulatory proprioceptive reflexes tend to produce abnormal chemical equilibria just as changes in the pulmonary proprioceptive reflexes do.

The relative importance of vascular reflexes in the control of breathing remains to be determined, but through the experiments of WINDER the intensity of the depression of breathing in relation to the endosinual pressure are now quantitatively established (see fig. 121 and 122). To quote "The animals (dogs) were just-adequately anesthetized with morphine (subcutaneously) and 
urethane (intramuscularly). After painstaking ligation of all efferent vessels of the carotid accessible without great risk of damage to nerve fibers, the remaining fine vessels, including the carotid gland bloodsupply, were embolised with lycopodium suspension (Heymans and Bouckaert, 1933). The gland was thus eliminated from any changing influences of carotid blood. The influence of changing pulmonary ventilation was eliminated by use of constant artificial ventilation. The inflation pump was connected with rebreathing tanks and recording spirometer, and adjusted to maintain an apparently normal breathing effort at normal carotid pressure. Mechanical freedom of

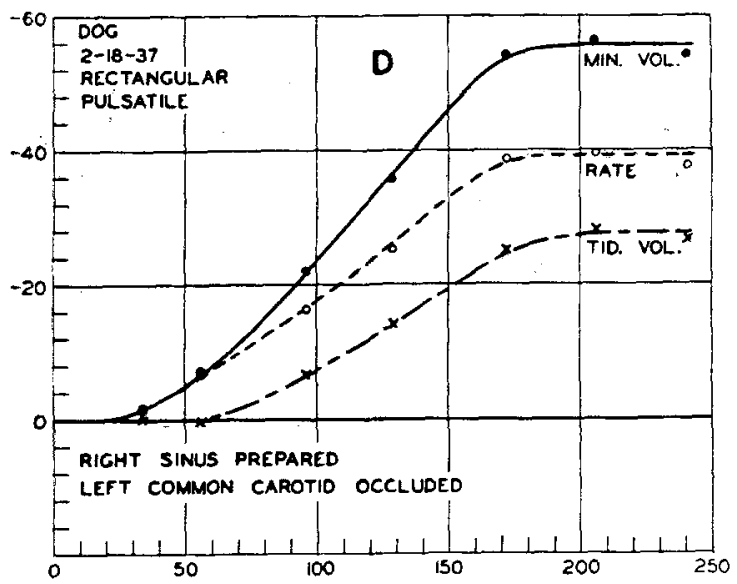

Fig. 122. Changes in frequency, tidal volume, and total volume of breathing in terms of percent of the standard breathing at zero endosinual pressure, are plotted on the ordinates against endosinual pressure on the abscisse. [WINder: Amer. J. Physiol. 122, 306 (1938).] pulmonary inflation and respiratory movements was afforded by unilateral open pneumothorax and ruptured mediastinum. By means of an adjustable resistance in the expiratory line, the passive elastic deflation could be regulated to maintain residual air in the lungs. The aortic pressure was controlled and carotid segment perfused, with blood of the same composition as that in the animal. For details of this arrangement see fig. 121 and legend. To eliminate unnatural tug on the carotid segment, which preliminary experiments demonstrated to be very important, the positions of the tubes connected with inflow (common carotid) and outflow (lingual artery) cannulae were adjusted by special universal supports. Breathing movements were recorded volumetrically by means of a complete body-plethysmograph connected with recording spirometer and amplifying pulley. The open pneumothorax was connected by means of a cannula and tubing to the exterior. Although the aortic-pressure compensator and constant pulmonary ventilation would have made aortic denervation unnecessary, the vago-sympathetic-aortic trunks were tied and cut to eliminate any venogenic or pulmonary proprioceptive reflex over the vagi and any carotid reflex changes in cerebral circulation via the cervical sympathetics."

Changes in frequency, tidal volume and total minute volume of breathing in terms of percent of the "standard" breathing at zero endosinual pressure, are plotted against endosinual pressures (fig. 122).

With the addition of WINDERs results to the literature it may now be stated that distention of the carotid sinuses lowers vascular constriction, blood pressure, heart rate, and pulmonary ventilation in similar quantitative 
ways. Under suitable experimental conditions, there is a delicate response of all of these functions to absolute pressures in the sinus. The activity of each reflex is represented by a sigmoid curve-inhibition beginning at approximately $20-50 \mathrm{~mm}$. Hg pressure, with maximum at approximately $200-250 \mathrm{~mm}$. $\mathrm{Hg}$ pressure, and increasing most rapidly at about 125-150 mm. Hg [KocH (134), Lim and Lu (135), Schneyer (136), Winder (133)]. The mechanism is accordingly most sensitive in the range of normal pressure changes. Schurdts (137) statement that "the intensity of respiratory response to alterations in endosinual pressure bore no constant relation to that of the circulatory" is held by WINDER to be based on experiments in many of which the aortic nerves were apparently intact and in all of which arterial pressure and pulmonary ventilation were uncontrolled. Whenever other important factors controlling breathing are kept constant, there is a quantitative reflex.

The similarity of the family of circulatory and respiratory curves established by graded distention of the carotid sinus is convincing evidence of the unity of action and purpose of the circulatory and respiratory systems. The fact that the circulatory reflexes arising in the carotid sinuses are more powerful than the respiratory reflexes need not call for a classification of the stretch reflex fibers into separate groups nor for elaborate central connections. Preponderating impingement of fibers in the circulatory centers would suffice. This concept is in conformity with the basic principle of nervous integration namely the sum total of impulses impinging on a nerve cell or center determines its degree of activity.

\section{The Influence of Chemical Equilibrium upon Respiratory Reflexes.}

Knowing that an altered reflex activity, produced by inflation of the lungs or of the carotid sinus, is capable of changing the chemical equilibrium of the body, one naturally inquires into the converse. Does an altered chemical equilibrium change the response to reflex stimulation? This question has been answered repeatedly in the affirmative.

Sherrington (61) states "A certain degree of asphyxiation much favors the elicitation of the scratch reflex." Graham Brown (138) finds that stoppage of breathing will bring out the scratch reflex when not present before. A fasting animal shows higher reflex excitability than does an animal well fed [SHerrington (77)]. Kaya and Starling (139) believe that the increased excitability of a spinal animal during asphyxia is due to lack of oxygen, rather than to excess of $\mathrm{CO}_{2}$. Experiments by Glazer $(140)$, WinkLer $(141,142)$ and GAX (143) on the effects of asphyxia, oxygen lack, carbon dioxide excess, intravenous injection of carbonate and bicarbonate, and hemorrhage have shown most striking effects. Spinal reflexes are increased by simple asphyxia 
produced by clamping the trachea, by oxygen lack, by hemorrhage, and by sodium carbonate. Carbon dioxide diminishes them. Sodium bicarbonate

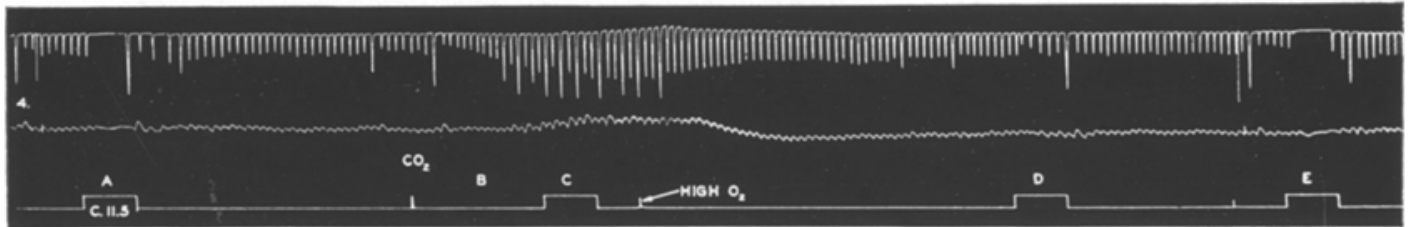

Fig. 123. Retardation of breathing produced by faradic stimulation of the central end of the vagus nerve at $\mathrm{A}$ is abolished by the central action of hypercapnia at $\mathrm{C}$. Partial recovery at $\mathrm{D}$. Complete recovery at E. [Geseld and Moyer: Quart. J. exper. Physiol. 25, 13 (1935).]
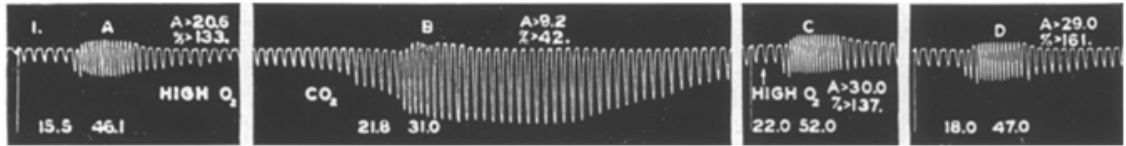

Fig. 124. Acceleration of the rhythm of breathing produced by sensory stimulation of the saphenous nerve at $\mathrm{A}$ is greatly diminished by hypercapnia at B. [GEseLL and MOYER: Quart. J. exper. Physiol. 25,13 (1935).]

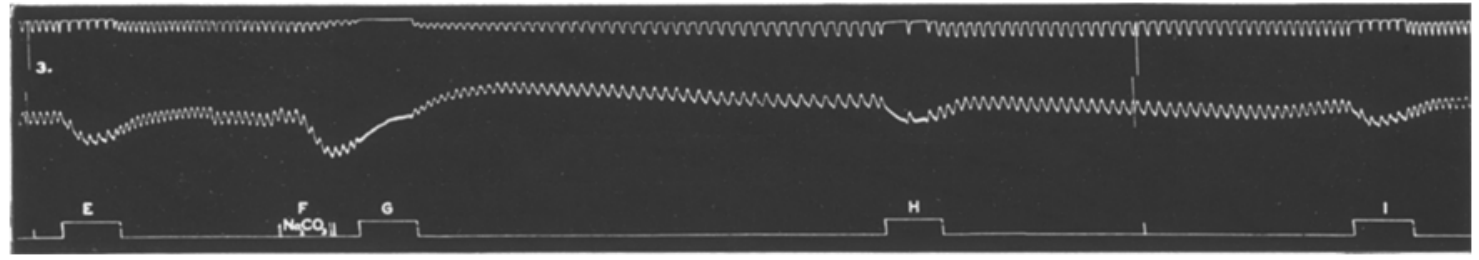

Fig. 125. The retardation of breathing produced by faradic stimulation of the central end of the vagus nerve at $\mathrm{E}$ is greatly increased by a proceeding injection of sodium carbonate at $\mathrm{G}$. Progressive recovery occurs at $H$ and I. [Gesell and Moyen: Quart. J. exper. Physiol. 25, 13 (1935).]
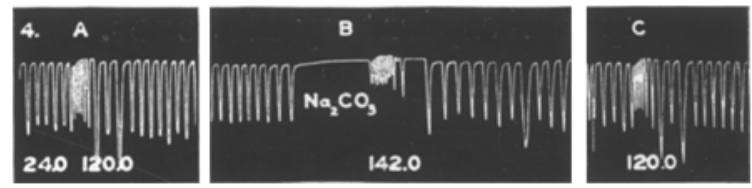

Fig. 126. The accelatered frequency of breathing produced by central stimulation of the saphenous nerve at $A$. is markedly increased with similar stimulation during carbonate apnea at B. Recovery at C. [GESELT and Moxer: Quart. J. exper. Phyisol. 25, 13 (1935).]
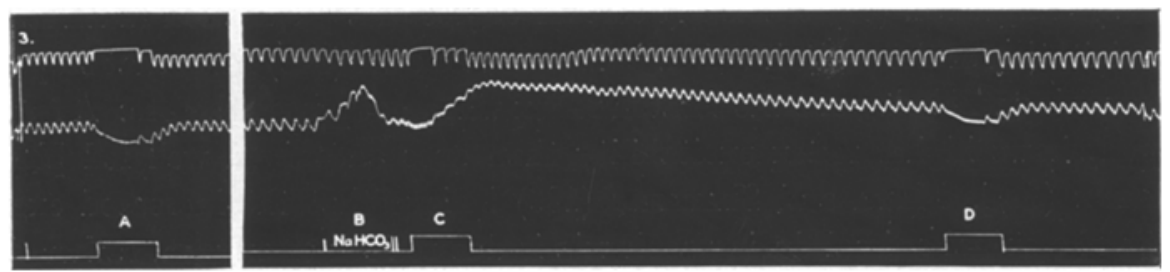

Fig. 127. The acid effects of intravenous injection of sodium bicarbonate weaken the vagal retardation reflexes like carbon dioxide in fig. 123. [Geselt and Moyer: Quart. J. exper. Physiol. 25, 13 (1935).]

may decrease or increase reflexes. It must be remembered that sodium bicarbonate theoretically produces a temporary increase of intracellular acidity. 
With these experiments as a lead Moyen and Gesell (79) studied respiratory reflexes elicited by electrical stimulation of the central end of the vagus and saphenous nerves during hypercapnia, hypocapnia (overventilation and intravenous injection of $\mathrm{Na}_{2} \mathrm{CO}_{3}$ ) and oxygen lack. Fig. 123 shows the diminution of vagal effects by hypercapnia. Vagal stimulation at A during eupnea produced a momentary cessation of breathing while at $\mathrm{C}$, during the height of hypercapnia, there were no indications of slowing. During the earlier stages of recovery, at $\mathrm{D}$, some retardation occurs again and at $\mathrm{E}$ the effects of vagal stimulation are back to normal. Fig. 124 shows a marked reduction in the acceleratory action of stimulation of the saphenous nerve by hypercapnia. During eupnea, at $A$, the frequency increases from 15,5 to 46 , during hypercapnia at B from 21,8 to 31 . As carbon dioxide is washed out again, $\mathrm{C}$ and $\mathrm{D}$, acceleration increases. Sodium carbonate, as might be expected, has just the opposite effects. It intensifies the retarding effects of vagal stimulation, often transforming them into apnea, as in fig. 125. And it increases the accelerating action of the saphenous nerve in a most striking way. In fig. 126 e.g. saphenous stimulation applied during eupnea increased the frequency of breathing from 24 to 120 per minute. Applied during apnea it increased the frequency from zero to 142 per minute.

Thinking in terms of blood $\mathrm{c}_{\mathrm{H}}$, sodium bicarbonate should also increase the intensity of reflexes. As fig. 127 shows, the reverse is the case. The vagal apnea produced at $\mathrm{A}$ is reduced at $\mathrm{C}$ by a previous injection of sodium bicarbonate. This, as we shall emphasize later, is in agreement with theory that bicarbonate momentarily turns the central nervous system more acid than normal. After some minutes of recovery at $D$ vagal stimulation is again more effective.

The effects of oxygen lack are often like those of sodium carbonate, namely an intensification of reflex activity. An example of such action is seen in fig. 128 in which breathing increases from 18 to 54 when the stimulus is applied during eupnea and from 22 to 71 when applied during oxygen deficiency. This effect as we shall see is also interpretable on the basis of acidity for there is considerable evidence indicating that oxygen lack, if the respiratory response is sensitive, will

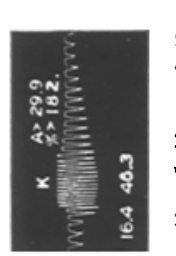

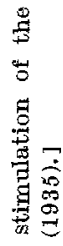

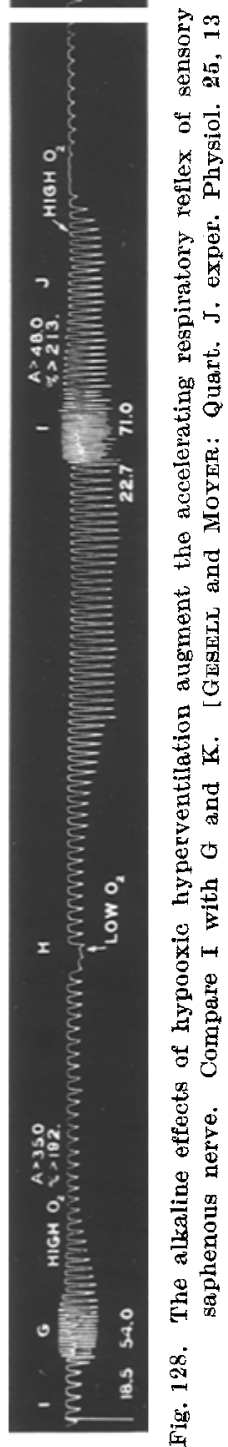
turn the body as a whole more alkaline than normal.

The effects of hypercapnia and hypocapnia were also studied on the vagal stretch reflex in which the adequate stimulation of inflation and deflation 
were used. Sodium carbonate and carbon dioxide were administered under three conditions, 1. during superinflation of the lungs, 2. during eupneic breathing with the lungs back to normal volume, and 3 . during a partially
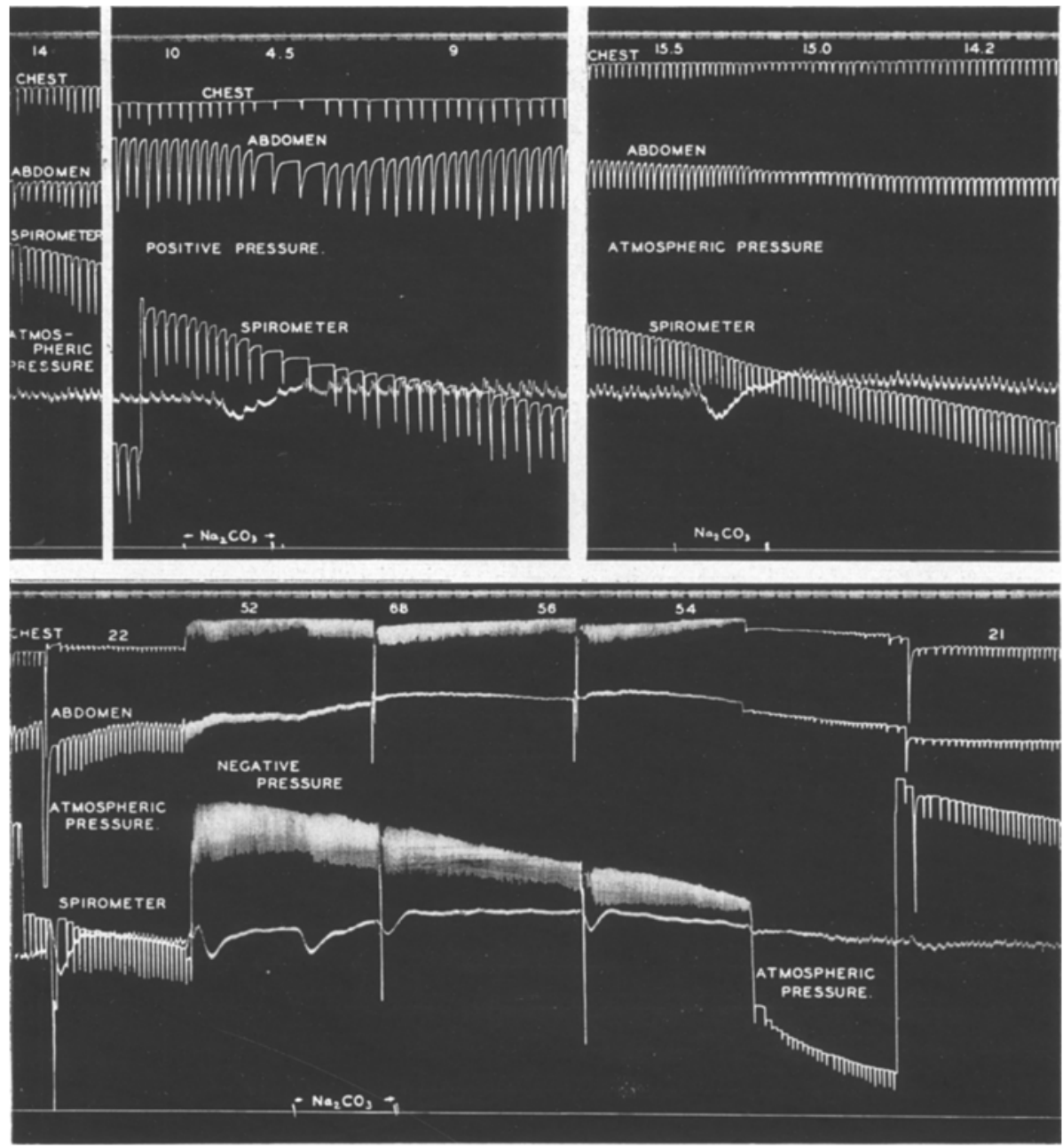

Fig. 129 to 132. Augmentation of reflex action by sodium carbonate. Inflation of the lungs produces a reflex retardation of breatbing from 14 per minute in fig. 129 to 10 in fig. 130 . This reflex retardation is augmented by intravenous injection of sodium carbonate, for the frequency of breathing decreases to 4.5 per minute. Similar injection under normal barometric condition in fig. 131 produces virtually no change in rhythm suggesting a near zero activity of the vagal stretch reflex. Defhation of the lungs in fig. 132 reflexly increases the frequency of breathing from 22 to 52 per minute. This acceleratory reflex activity is intensified by the intravenous injection of sodium carbonate as is indicated by the rise in frequency of breathing from 52 to 68 per minute. [GEsELL and MOYER: Quart. J. exper. Physiol. 25, 13 (1935).]

deflated condition of the lungs produced by counterweighting the spirometer (see fig. 129 to 132). On referring to fig. 129 it will be seen that the $\operatorname{dog}$ is breathing at a frequency of 14 per minute. The lungs are then inflated (fig. 130) and the frequency falls to 10 indicating that the vagal stretch reflex is active. Sodium carbonate now reduces the frequency to 4.5 per minute. The animal 
is then allowed to recover (fig. 131) and the excess pressure is removed from the lungs and sodium carbonate is again injected but with virtually no diminution in frequency of breathing. Only amplitude is decreased. The lungs are
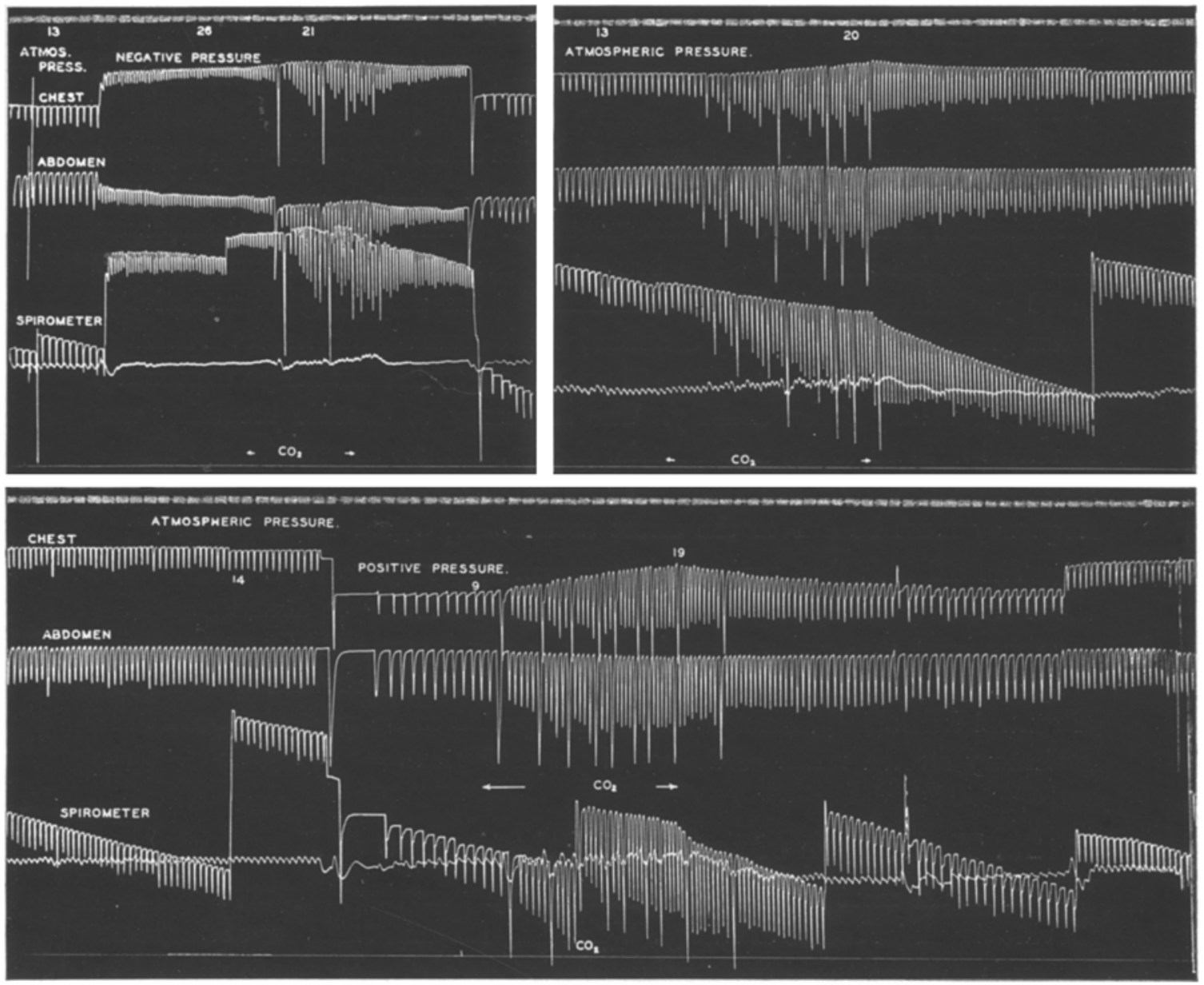

Fig. 133 to 135 . Weakening of reflex activity by hypercapnia. An acceleratory reflex set up by deflation of the lungs in fig. 133 increases the frequency of breathing from 13 to 26 per minute. Administration of carbon dioxide cuts this acceleration to 21 indicating that a weakening of the reflex had occurred. A retardation reflex set up by superinflation of the lungs in fig. 135 decreases the frequency of breathing from 14 to 9 per minute. Administration of carbon dioxide weakens this retardation effect as indicated by an increased frequency of breathing to 19 per minute. When carbon dioxide was given during normal barometric conditions the frequency of breathing increased from 13 to 20 per minute. This increase is probably due to a weakening of a prevailing vagal retardation reflex and to a chemical stimulating action. [GESELL and Moyer: Quart. J. exper. Physiol. 25, 13 (1935).]

then deflated in fig. 132 and breathing increases from 22 to 52 per minute as a result of a different set of reflexes. Sodium carbonate then increases this newly established acceleration from 52 to 68 per minute. It is, therefore, concluded that carbonate strengthens the activity of prevailing respiratory reflexes, both retardation and acceleration. The fact that little change in 
frequency occurred upon injection of sodium carbonate during eupneic breathing would indicate that the proprioceptive reflex activity was at a very low level. More commonly carbonate will decrease the frequency of eupneic breathing indicating that the vagal stretch reflex possesses residual activity even after the lungs have collapsed to their expiratory position. This tallies with Adrians findings that many vagal fibers fire continually during the expiratory as well as inspiratory phase.

The observations on inflation and deflation of the lungs were then repeated with the integrating system on the acid side of normal. Carbon dioxide was administered in the place of sodium carbonate. When given during pulmonary inflation, which normally slows breathing, the frequency increased from 9 to 19 per minute, indicating a diminution of the prevailing reflex activity. When given during pulmonary deflation, which normally accelerates breathing, the frequency decreased from 26 to 21 per minute again indicating a diminution of the prevailing reflex activity. The frequency of eupneic breathing was increased from 13 to 20 , a substantial change, but not as great as the acceleration produced by carbon dioxide during lung inflation. This increase in frequency must be due to the direct accelerating action on the center.

The old idea that sodium carbonate produces apnea by a reduction of the excitability of the respiratory center must obviously be abandoned when so many important reflex activities are actually intensified. The production of apnea by hypocapnia, which originally gave rise to the old idea of depression of central excitability, must rather be interpreted as an augmentation of the expiratory stretch reflex of the vagal proprioceptors.

\section{The Nature of the Chemical Stimulus of Breathing.}

From the most primitive to the most highly developed forms, oxidations provide the major source of energy. When they fail, non-oxidative or anaerobic energy is thought to fill the gap by a varied train of processes in which free energy and lactic acid are liberated. Along with chemical changes more recently discovered, lactic acid shifts back into the precursor state at the expense of oxidative energy during recovery. The cell is again prepared to supply energy, either by the aerobic or anaerobic way. Whichever method is employed, acid is formed, carbon dioxide from oxidations and lactic acid from anaerobic metabolism. Furthermore, the greater the intensity of either type of metabolic activity, the greater is the amount of acid formed and the greater is the need for respiratory correction. Because increased hydrogen ion concentration depresses oxidations, increased oxidations, even during oxygen plenty, must automatically create a barrier against oxidations unless the excess carbon dioxide is removed. Thus carbon dioxide becomes a logical automatic regulator of breathing under any situation. 
Oxygen lack impairs oxidations in two ways, directly, due to lessened availability of oxygen and indirectly, due to the accumulation of anaerobic acid. It, therefore, offers double possibilities as an automatic regulator of breathing. Attempts to harmonize the direct effect of oxygen deficiency with respiratory stimulation have so far met with failure. Only the indirect acid effects seem to meet the requirements of theory. For example, the increased oxidations resulting from an elevation of temperature, increase breathing just as reduced oxidations do from oxygen deficiency. On the other hand superoxidations produced by readministration of room air after a period of oxygen deficiency will often stop breathing completely. Some factor other than intensity of oxidations seems to be the cause of the changes in breathing. All evidence points to acidity.

The concentrations of hydrogen ions in the blood cannot possibly be the determining factor of any importance for there are far too many gross exceptions to that concept [GeseLx $(1,146$ and 147)]. The decreased hydrogen ion concentration of the blood in hyperventilation during oxygen deficiency, of cyanide poisoning, of intravenous injection of sodium bicarbonate are most important examples. The recognition of inconsistancies of this kind inevitably led to the cellular acidity theory of respiratory control [WINTERstein (148); Geselu $(1,146,147)]$. During the temporary confusion following on the discovery of the reflex chemical mechanism of control the acid theory suffered in prestige. But as we shall try to show, the arguments in favor of acidity as the primary factor of chemical control are as strong today, in full knowledge of the peripheral and central chemical control, as they were before the discovery of the function of the carotid bodies.

The most important contradictions to the older or blood acidity theory were the lack of parallelism of breathing and blood $c_{\mathbf{H}}$ during oxygen deficiency and following intravenous injection of sodium bicarbonate. Since lactic acid forms in greater abundance during oxygen lack than does carbon dioxide during oxygen plenty, theory demands, other things remaining constant, that a cell subjected to oxygen deficiency turns more acid than normal. Not only is lactic acid formed more rapidly, but buffer base is diminished in proportion by the reaction between lactic acid and sodium bicarbonate and free carbon dioxide liberated, which constitute a most effective combination for increasing $c_{\mathrm{H}}$. When oxygen is resupplied under these conditions of high concentration of sodium lactate, lactic acid shifts back into the precursor state, thereby liberating free sodium. The combination of sodium with carbon dioxide increases the denominator of the $\frac{\mathrm{H}_{2} \mathrm{CO}_{3}}{\mathrm{NaHCO}_{3}}$ ratio and at the same time decreases the numerator, thus lowering the hydrogen ion concentration. If the carbon dioxide which is liberated during the anaerobic period escapes from the cell, oxidative recovery will theoretically decrease the hydrogen ion 
concentration below normal because the amount of lactic acid which disappears (by oxidation and by return to the precursor state) is greater than the amount of carbon dioxide formed. The implications of these concepts applied to the oxygen sensitive chemoceptors are capable of explaining the hyperventilation of oxygen deficiency and the apnea and return to normal breathing on readministration of room air. Supporting arguments will be presented below.

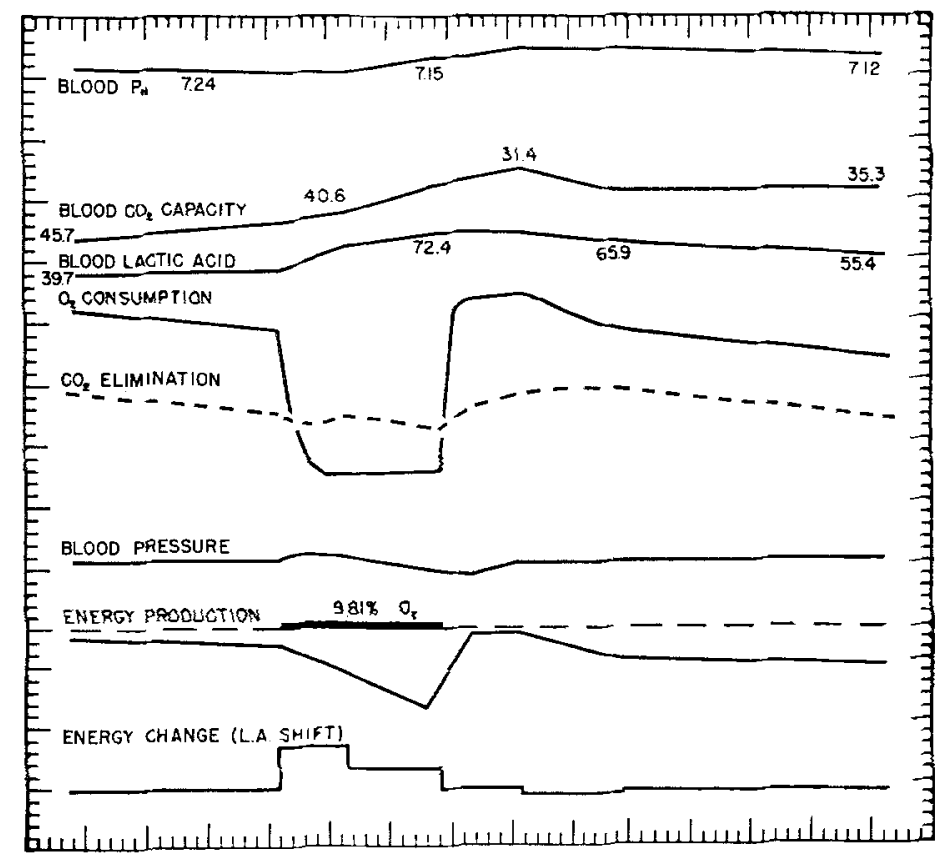

Fig. 136, An analysis of the outstanding respiratory changes occurring during a period of oxygen deficheneyd recovery in which room air and a $9.8 \%$ oxygen mixture in nitrogen were administered by uni. form artificial ventilation. The harmful effects of low oxygen pressures in the inspired air in the absence of compensatory ventilation are to be compared with the harmless effects when normal compensation is allowed in fig. 137. For further analysis see text. [Gesel., Krotger, Gorham and Berrthat: Amer. J.

Physiol. 94, 300 (1930).]

A comparative study of the chemical changes produced by oxygen deficiency during uniform artificial pulmonary ventilation and during normally controlled ventilation will help to elucidate this point [Geseli, Krueger, Gorham and Bernthal (151); and Gesell, Krueger, Nicholson, BrassFreld and Pelecovicm (152)]. It will indicate the possible origin of chemical control and how the organism profits from normally controlled breathing (fig. 136 and 137). The damage sustained in shifting to a $9.8 \%$ oxygen mixture during uniform ventilation in fig. 136 is revealed in the reduction of oxidations and most probably in the total free energy available. In view of the controversies now existing in the field of musele chemistry and the dearth of similar studies in other tissues, we prefer to avoid a quantitative evaluation of the anaerobic energy liberated during oxygen deficiency. With that reservation there can be no harm in including our energy curves as originally 
computed for whatever information they may impart to those conversant with this most difficult subject. What we really wish to stress are the changes in acid base equilibrium consequent upon the interaction of aerobic and anaerobic metabolism. The actual findings on blood lactic acid, blood carbon dioxide capacity, blood $c_{\Pi}$ and carbon dioxide pressures, however represent information in this most important phase of breathing which cannot be dismissed

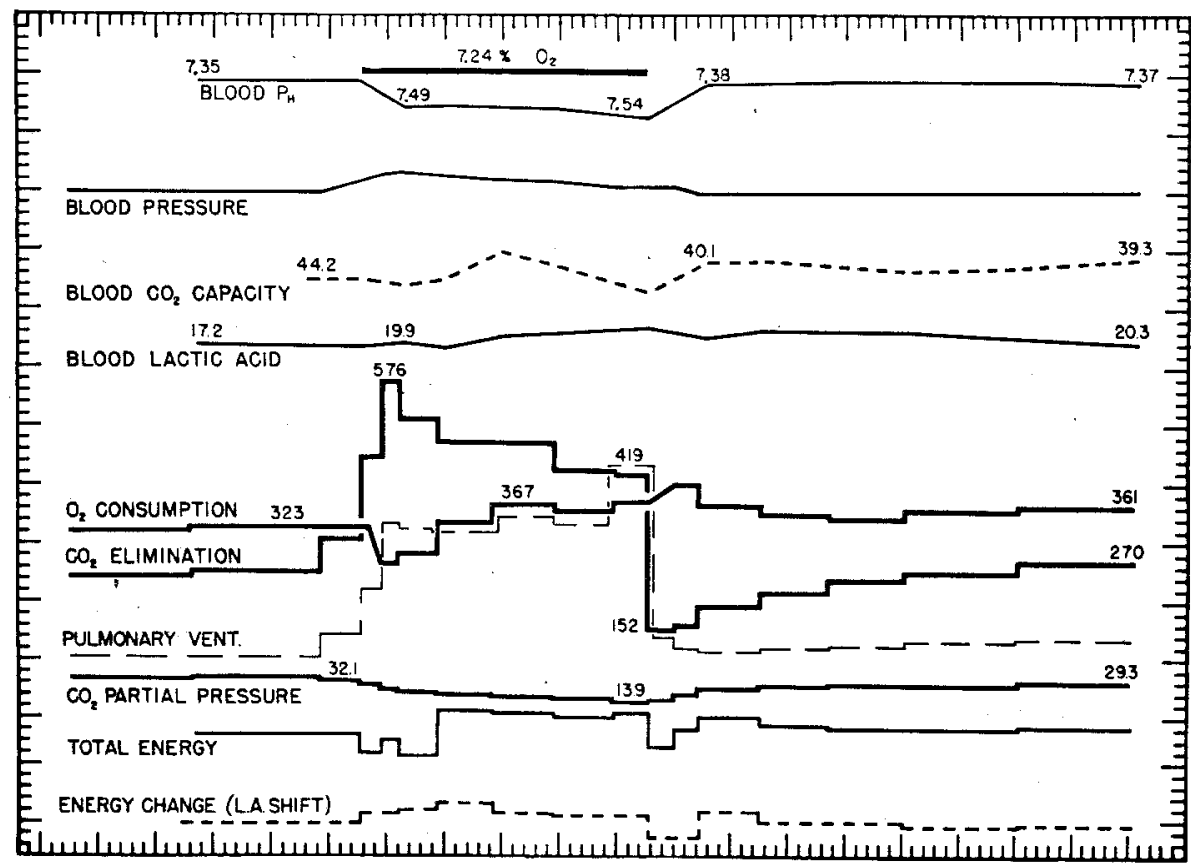

Fig. 137. A similar analysis of outstanding respiratory changes occurring during a period of oxygen deficiency and recovery when normal compensation of pulmonary ventilation was allowed. For further details consult text. [Gesell, Krugger, Nicholson, Brasgfield and Perecovich: Amer. J. Physiol. 100, $202(1932)$.]

regardless of the views which may be held in other details. (In studying these results allowance should be made for the slow deterioration of the animal. Oxygen consumption, carbon dioxide elimination, energy production [as computed] and blood pressure all show a downward inclination upon which the effects of temporary oxygen deficiency are superimposed.) Oxygen consumption falls abruptly, yet carbon dioxide elimination increases above normal. Since pulmonary ventilation is unchanged, the cause must be sought either in an improved transport resulting from augmented volume flow of blood or to acid base changes in the tissues. The rapid increase of lactic acid in the blood indicates a rapid formation in the tissues and the increase of the respiratory quotient from 0.73 to 1.55 indicates that free lactic acid is reacting with sodium bicarbonate increasing the carbon dioxide pressure in the blood. It is, therefore, reasonable to assume that the tissues as well as the blood are turning acid. 
On readministration of room air, oxidation increased abruptly to a supernormal level, indicating that the body had contracted an oxygen debt. Carbon dioxide is eliminated in excessive amounts, blood $\mathrm{CO}_{2}$ capacity, blood lactic acid and blood $c_{\mathrm{H}}$ show tendencies towards recovery. Free energy, as computed on the basis of oxidations and energy freed by anaerobic liberation of lactic acid, shows a decided deficit for the combined period of oxygen deficiency and recovery.

These results may now be compared with those of oxygen deficiency when pulmonary ventilation is normally controlled (see fig. 137). First of all, pulmonary ventilation increases and oxygen consumption is only momentarily lower than normal and as ventilation continues to increase oxygen consumption mounts to supernormal values. Excessive elimination of carbon dioxide and the relatively small amount of lactic acid formed seem to be the important factors in this remarkable adjustment. The respiratory quotient increases from 0.77 to 2.18 despite the small formation of lactic acid. This increase of the respiratory quotient is much greater than that occurring during constant ventilation and is attributed primarily to the superventilation witnessed by the reduction of carbon dioxide pressure in the expired air from $34 \mathrm{~mm}$. $\mathrm{Hg}$ to $14 \mathrm{~mm}$. $\mathrm{Hg}$.

As a result of the small outpouring of fixed acid into the blood stream, the blood carbon dioxide capacity is but little changed, and the blood $\mathrm{p}_{\mathrm{H}}$ remains high due to the low carbon dioxide pressure. The well sustained elevation of blood pressure indicates a good condition of the dog which is logically referable to the adequacy of the supply of energy during the period of oxygen stress. Actually the free energy during this period is higher than that of normal and this is due almost entirely to increased oxidations. The moment room air is readministered pulmonary ventilation returns virtually to normal, indicating that respiratory stimulation is also back to the original value. This is interesting because oxygen consumption continues above basal levels, but if one will look to the respiratory quotient and the diminution of lactic acid, a possible explanation is seen for the unexpectedly low ventilation. The extraordinary low respiratory quotient of 0.40 indicates a chemical retention of carbon dioxide by the body. There are two reasons for this retention. One is the liberation of free sodium as the lactic acid shifts back to the precursor state. That factor, however, is very small in this experiment. The other is the preceding overventilation of the body during the period of oxygen deficiency in which carbon dioxide was removed faster than it was formed by oxidation plus liberation in the reaction of lactic acid with sodium bicarbonate. That loss of carbon dioxide must now be made up by retention of newly formed oxidative carbon dioxide before a steady state is reestablished. Herein, we believe, rests the main explanations of respiratory adjustment during oxygen lack. By elevating the $p_{\mathrm{H}}$ of the tissues during oxygen 
deficiency, oxygen consumption is held at high levels despite a lowering of the partial pressure.

The oxidative energy liberated during the period of stress and recovery, being actually higher than normal, stands in marked contrast to that of uniform ventilation. A need for this extra energy is seen in several requirements entailed by oxygen lack 1. Extra mechanical energy for the movement of air 2. Extra thermal energy for warming a greater amount of inspired air 3. Extra thermal energy for saturation of the inspired air with water vapor 4 . Extra energy for the liberation of carbon dioxide from the blood into the alveolar air 5. Extra mechanical energy for increased cardiac output 6. Extra energy for the repair of possible chemical damage etc. The energy required for items $1,2,3$ and 4 was found by computation to be less than the actual amount of excess energy. It was, therefore, suggested that repair of cellular damage may be an important factor in experiments of this kind.

Under more ideal conditions than those of acute experimentation, such as life at high altitudes, there are no chemical signs of oxygen stress, and the failure of lactic acid to appear in the blood stream has been used by some as an argument against an acid mechanism of control during oxygen lack. But it must be remembered that under such conditions the machinery of breathing is working as evolution may have intended, that is to eliminate all harmful effects of the scarcity of oxygen. The cellular acidity theory demands that only those structures designed to correct against oxygen lack withstand the impact of oxygen shortage. If these structures be infinitesimally small, as compared with the body as a whole this would add an infinitesmally small amount of lactic acid to the blood and we should be unable to find an increased amount of lactic acid in healthy individuals acclimated to high altitude. As it has finally turned out, the tiny collections of chemoceptors found in the carotid and aortic bodies are the regulators of breathing against oxygen lack. They alone stand the brunt of impaired oxidations and by their activity protect the body as a whole. As we shall show, the respiratory center actually becomes acapnic with anoxic hyperpnea for when HenINGs nerves are blocked breathing stops. But this is not meant to imply that the center is excluded in the general scheme of the cellular acid theory of respiratory control. Like the chemoceptors it responds to changes in acidity. The elucidation of a more complete application of the cellular reaction theory will follow.

Another puzzling point in the blood acidity theory of respiratory and circulatory control were the opposite effects of the intravenous injection of sodium carbonate and sodium bicarbonate. Both lower the $c_{H}$ of the blood, but carbonate produces apnea and lowers the blood pressure while bicarbonate produces hyperpnea and elevates blood pressure. One important point overlooked in ascribing a specific action to the $\mathrm{HCO}_{3}$ ions was the opposite effect of these salts upon $\mathrm{CO}_{2}$ pressures of the blood and tissues. Theoretically, 
bicarbonate should turn the cells more acid, at least temporarily, by driving carbon dioxide into them, while carbonate should turn them more alkaline by withdrawing carbon dioxide from them. The principle is most simply demonstrated in stratification experiments. For example one may layer two

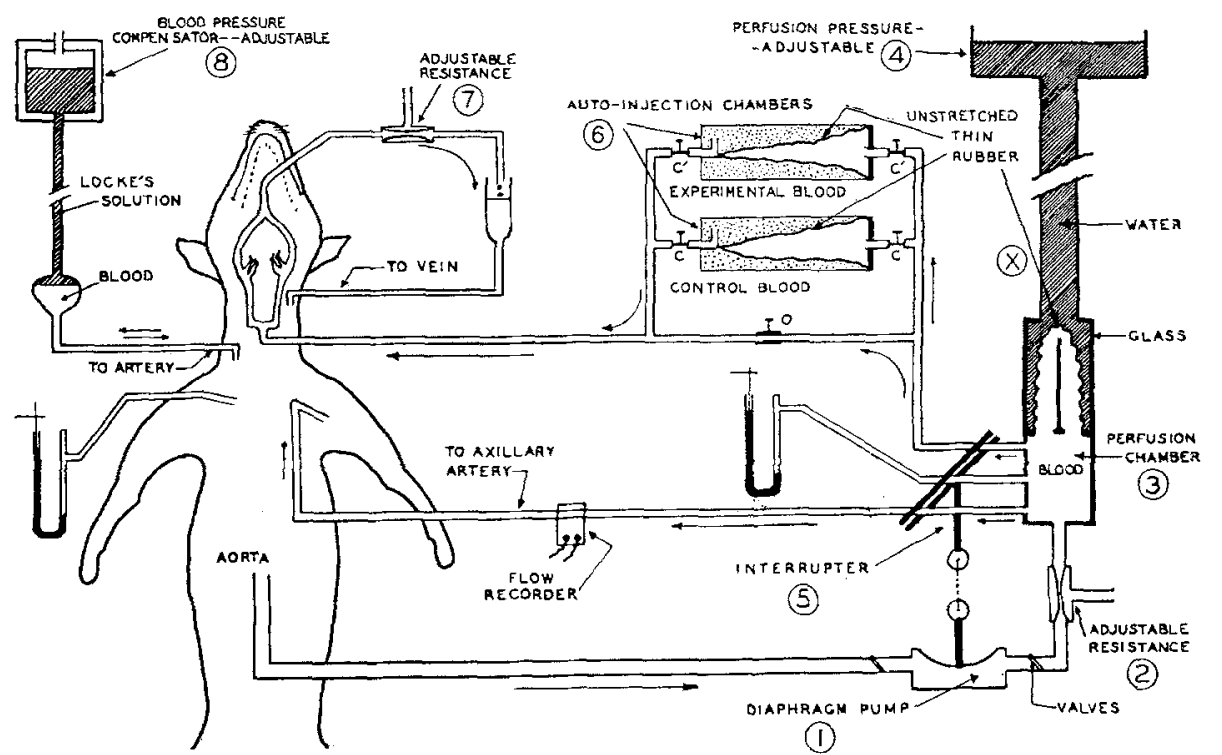

Fig. 138a. General schema of apparatus for perfusion of carotid body region and axillary artery. The diaphragm pump, 1, transfers blood from the aorta into the perfusion chamber, 3. The resistance, 2, is so adjusted that the animal is prevented from foreing blood into chamber 3 independently of the pump during temporary rises of aortic blood pressure. Perfusion pressure is determined by the level of the water reservoir 4 and is transmitted to the blood in chamber 3 through the thin rubber membrane, $X$. Blood is distributed from perfusion chamber 3 to the carotid body and axillary artery perfusion circuits through the separate outlets shown. The outflow from chamber 3 is made pulsatile by the interrupter, 5. Pump and interrupter are driven by a single motor and are so timed that the outlets from ehamber 3 are blocked. during the forward stroke of the pump and are opened only during the back stroke of the pump. In the carotid body perfusion eircuit, normal blood from chamber 3 can be replaced by blood from the autoinjection chambers, 6, without interruption of flow or alteration of pressure, by simultaneously closing clamp 0 and opening clamps $\mathrm{C}^{\prime}$ or $\mathrm{C}$. Endosinusal pressure and rate of flow are adjustable both at pressure source, 4, and at carotid outflow resistance, 7. Both the rate and the stroke amplitude of the pump, 1 , are adjustable while the latter is in operation. Stroke amplitude is continually adjusted in such a way that the blood removed from the animal at the aorta is exactly equal to that returned through the perfasion eircuits, deviation from such balance being shown by collapse or inflation of the rubber membrane, $X$. The systemic blood pressure compensator, 8 , is connected to the cardiac end of any large artery. The whole apparatus is submerged in a constant temperature water bath at $37.5^{\circ} \mathrm{C}$. [Bernthal: Amer. J. Physiol. 121, 1 (1938)].

hydrochloric acid solution of the same $\mathrm{p}_{\mathbf{H}}$ to one of which $\mathrm{NaCl}$ has been added. With the aid of a color indicator added to these solutions it is seen at the interface that the solution without $\mathrm{NaCl}$ turns acid while the other turns less acid. Similar experiments are readily performed with sodium bicarbonafe buffer mixtures. With proper concentrations one solution distinctly the more alkaline of the two will turn the other more acid. This all happens without the intervention of a selective membrane. In biological experiments the important role of cell membranes in acid base equilibria allow a ready demonstration of phenomena similar to those of stratification. With the aid of two 
electrodes, one in the blood and the other in the cerebrospinal fluid these principles may be tested. Injection of carbonate produces the expected reduction of $c_{H}$ in both blood and cerebrospinal fluid while bicarbonate causes the same fall in the blood $c_{\mathrm{H}}$ but a distinct rise in the cerebrospinal fluid $c_{\mathrm{H}}$. When all chemoceptors are denervated intravenous injection of carbonate decreases and bicarbonate increases ventilation and blood pressure, thus indicating that the changes in breathing under these conditions where the center alone is involved cannot be correlated with accompanying changes in
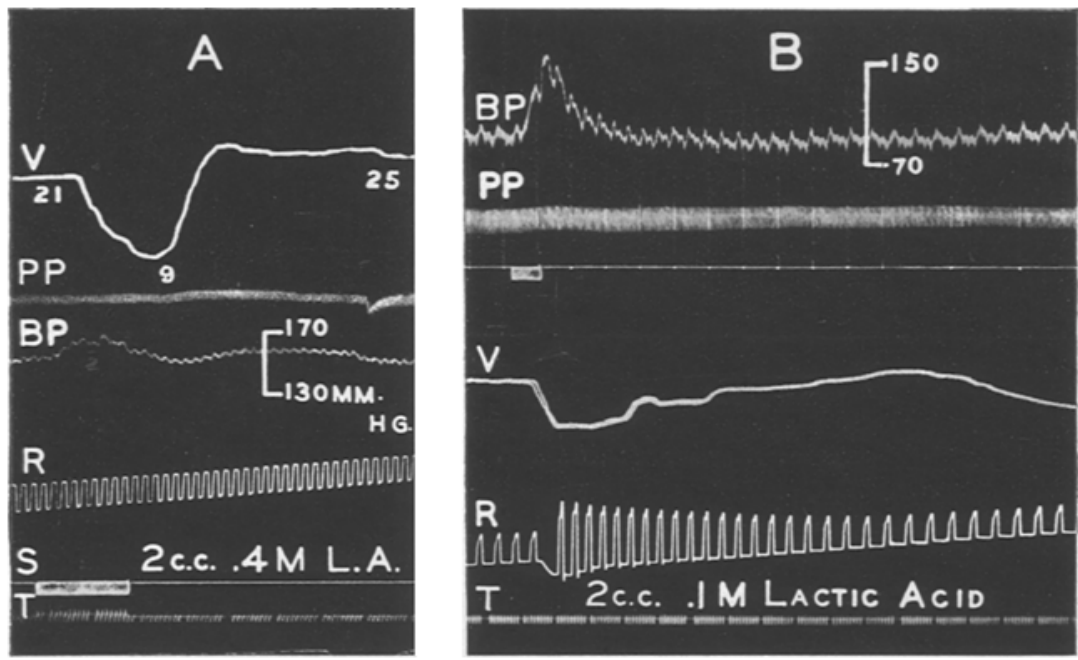

Fjg. 139 A. Increased activity of the chemoceptors produced by addition of lactic acid to the blood perfusate circulating through the vascularly isolated carotid body. Vascular constriction, the index of. chemoceptor activity, diminishes the flow of blood from 21 to $9 \mathrm{co}$. per minute (see record $V$ ). Ventilation is artificial and uniform [Bernthal: Amer. J. Physiol. 121, 1 (1938).]

Fig. $139 \mathrm{~B}$. Increased chemoceptor activity produced by lactic acid as indicated by increased vasoconstriction and pulmonary ventilation. These results conform with an expected increased acidity of the chemoceptors. [Bernthal: Amer. J. Physiol. 121, 1 (1938).]

blood $\mathrm{c}_{\mathbf{H}}$, but rather with the acidity of the "center". The role of acidity of the center to anoxemia will be considered below.

Bernthal (152) has studied the activity of the vascularly isolated carotid body as affected by sodium carbonate, sodium bicarbonate, carbon dioxide and lactic acid with the equipment illustrated in fig. 138. By eliminating the confusing and conflicting effects of physiological changes in blood pressure and pulmonary ventilation, he has been able to gauge the activity of the carotid body with accuracy. The indices of activity employed were volume flow of blood and pulmonary ventilation. Vascular constriction or decreased blood flow and hyperpnea indicate increased chemoceptor activity.

Hypercapnic blood decreased the brachial flow of blood. Lactic acid added to the blood had the same effects (see fig. 139A). When natural pulmonary ventilation was allowed breathing increased as well (see fig. 139 B). 
Sodium carbonate increased volume flow (fig. 140A) and decreased pulmonary ventilation (fig. 140B) indicating a decreased carotid body activity.
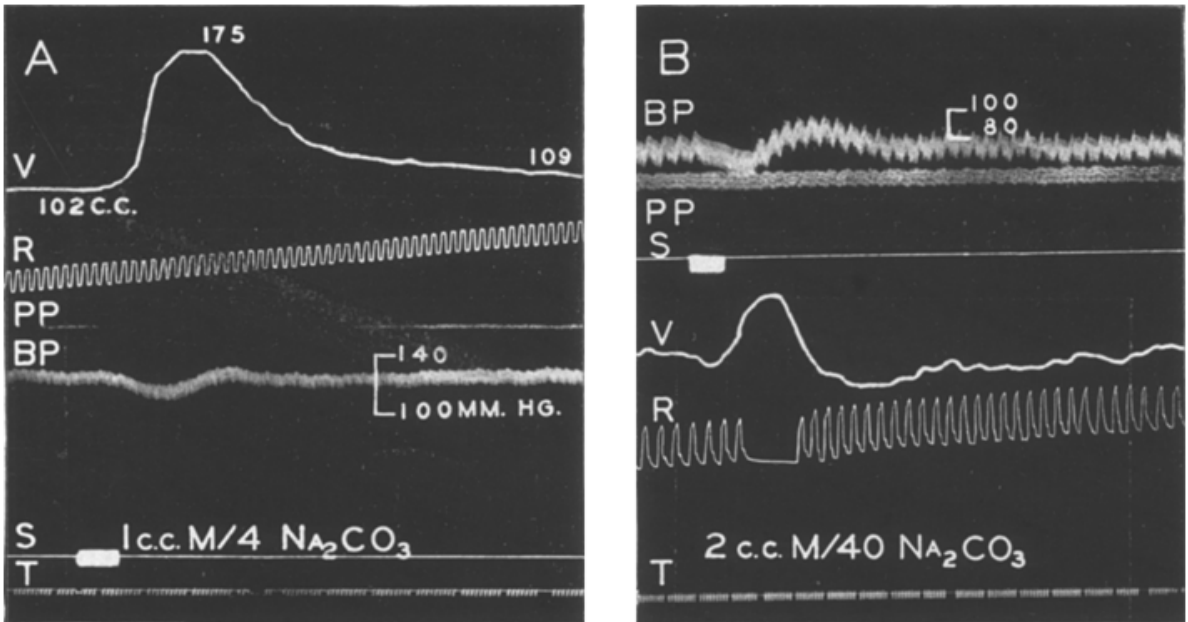

Fig. 140 A. Sodium carbonate added to the blood circulating through the carotid body during uniform artificial ventilation reduces the activity of the carotid body as evidenced by the diminution of tonic vasoconstriction. The volume flow of blood through the leg was increased from 102 cc. to $175 \mathrm{cc}$. per minute. [Bernthal: Amer. J. Physiol, 121, 1 (1938).]

Fig. 140 B. Sodium carbonate acting locally at the carotid body reduces breathing as well as vascular activity. These resnlts conform with an expected decreased acidity of the chemoceptors. [BERNTHAL: Amer. J. Physiol. 121, 1 (1938). ]
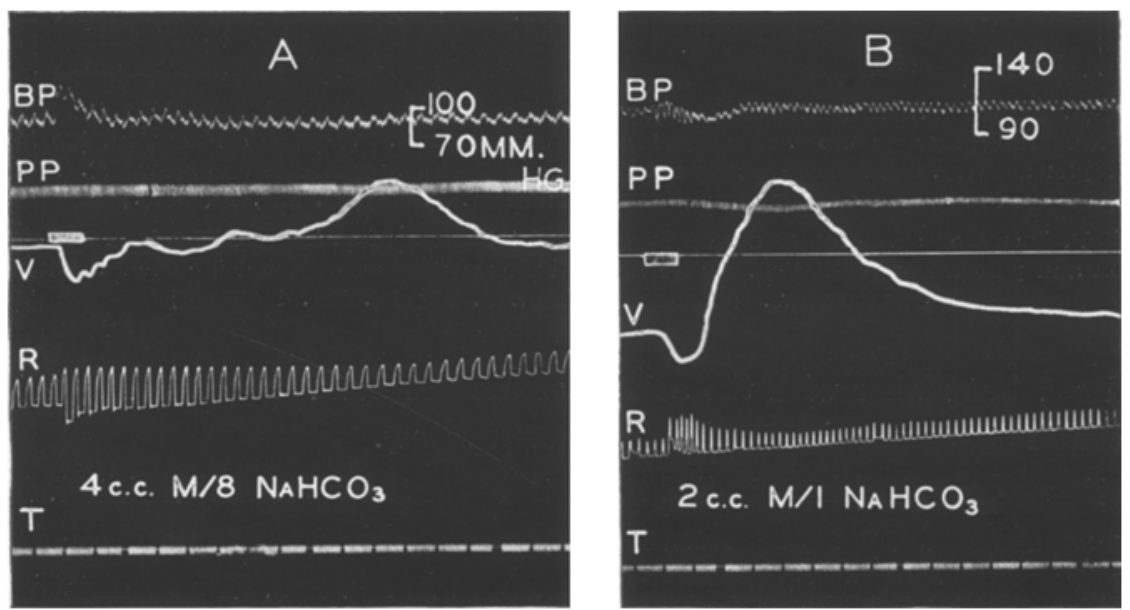

Fig. $141 \mathrm{~A}$ and $\mathrm{B}$. The temporarily increased vasoconstriction and pulmonary ventilation produced by a local circulation of sodium bicarbonated blood through the carotid body conforms with an expected temporary aeidified condition of the chemoceptors. [BERNTHaL: Amer. J. Physiol. 121, 1 (1838).]

Bicarbonate on the other hand produced a temporarily decreased volume flow of blood and increased pulmonary ventilation indicating increased carotid body activity (fig. $141 \mathrm{~A}$ and $\mathrm{B}$ ).

Brassfield and Hong (153) have supplied further data on intracellular acid base equilibrium by the studies on salivary $\mathrm{p}_{\mathrm{H}}$ in which they recorded 
continuous changes of blood and saliva with the aid of glass electrodes. The administration of carbon dioxide is seen to increase the hydrogen ion concentration of both the blood and saliva (fig. 142) and artificial overventilation of the lungs decreases the $\mathrm{c}_{\mathbf{H}}$ of both blood and saliva (fig. 143). Intravenous

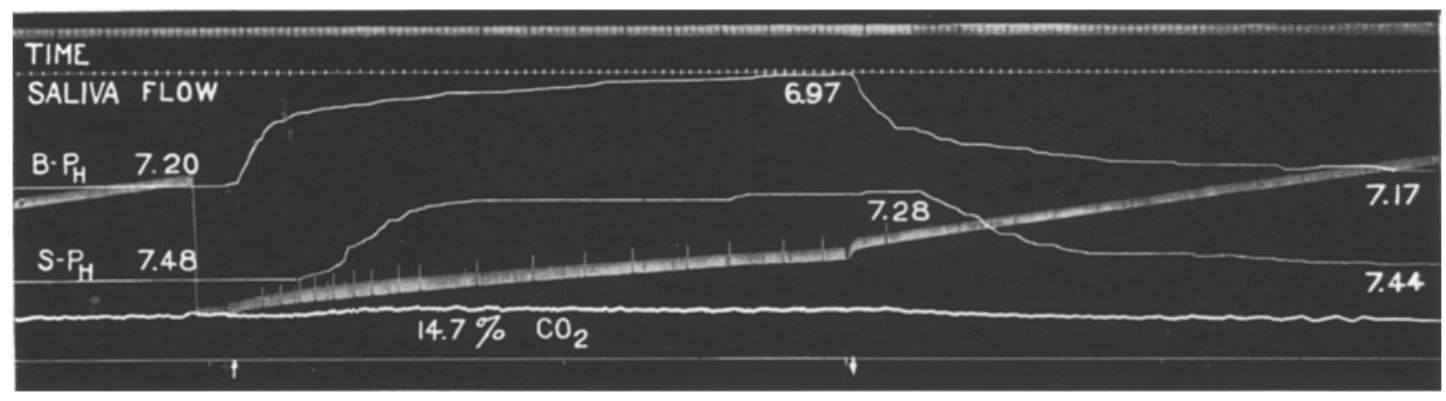

Fig. 142. The parallel increase of acidity of the blood and saliva produced by an administration of $\mathrm{CO}_{2}$ expected from the simple laws of diffusion suggest that salivary $c_{H}$ may be a fairly reliable index of acidity changes within the salivary glandeells during hypercapnia. $\mathrm{B} . \mathrm{P}_{\mathrm{H}}$ blood $\mathrm{p}_{\mathrm{H}}$. $\mathrm{S}$. $\mathrm{P}_{\mathrm{H}}$ saliva $\mathrm{p}_{\mathrm{H}}$. Both curves continuously recorded with the glass electrodes. [BRAssfieid and Behrman: Amer. J. Physiol. 122, 21 (1938).]

injection of lactic acids increases the $c_{\mathrm{H}}$ of the saliva (fig. 144) while sodium carbonate decreases it (fig. 145). In the light of these results it is most significant that sodium bicarbonate, which if it migrated freely across the salivary cell membrane would produce an invariable alkaline effect upon the

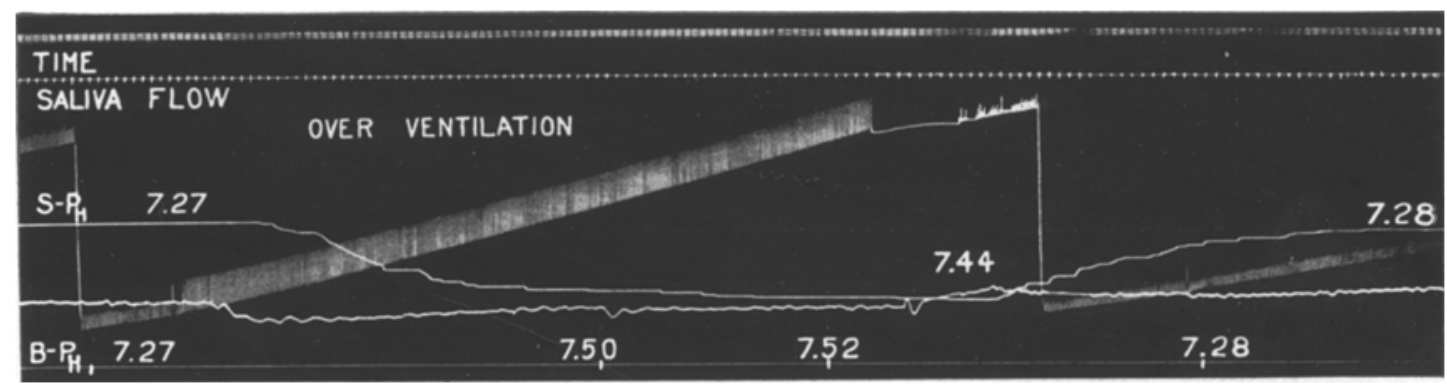

Fig, 143. Artificial over-ventilation increases saliva and blood $p_{H^{*}}$ The continuous saliva $p_{H}$ curve was recorded with the glass electrode. Blood $p_{\mathrm{HI}}$ was determined from blood samples. The parallel changes in $p_{H}$ are referable to a free diffusion of carbon dioxide from the saliva and blood. [BrassFresD and Behrman: Amer. J. Physiol. 122, 21 (1938).]

saliva nevertheless produces three effects 1 . an increased $c_{H}$ of the saliva (fig. 146) 2. no change (fig. 147) and 3. a decreased $c_{\mathbf{H}}$ (fig. 148). This variability of results agrees with the variability of effects of sodium bicarbonate on carotid body activity and central reflex activity and thus supports the acid interpretation of the stimulation of breathing by sodium bicarbonate.

When LundsGaARD $(154,155,156$ and 157$)$ demonstrated that monoiodoacetic acid prevents the usual formation of lactic acid during oxygen deficiency, he effered an opportunity of inquiring more specifically into the role 
of acidity as a stimulus in hypooxic hyperpnea. On the basis of his work it may be reasoned that if oxygen deficiency increases pulmonary ventilation in the absence of glycolysis, lactic acid may be dismissed as the normal source

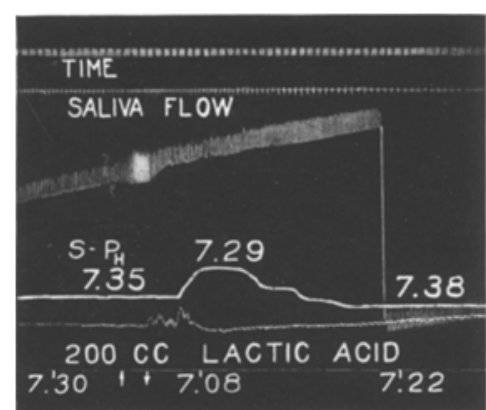

Fig. 144. Intravenous injection of lactic acid increases the acidity of the outflowing saliva and increases pulmonary ventilation. [BRAssfresd and Behrman: Amer. J. Physiol. 122, 21 (1938).]

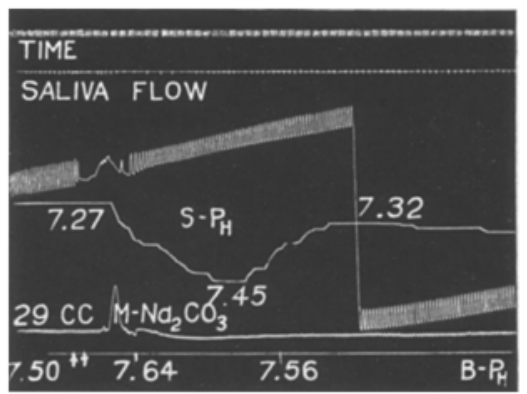

Fig. 145. Intravenous injection of sodium carbonate decreases the $c_{H}$ of the saliva. [BRASgFiELD and Behrman: Amer. J. Physiol. 122, 21 (1938).]

of stimulation. On the other hand should hyperpnea fail to appear during oxygen lack yet be elicitable by carbon dioxide excess, lactic acid may then
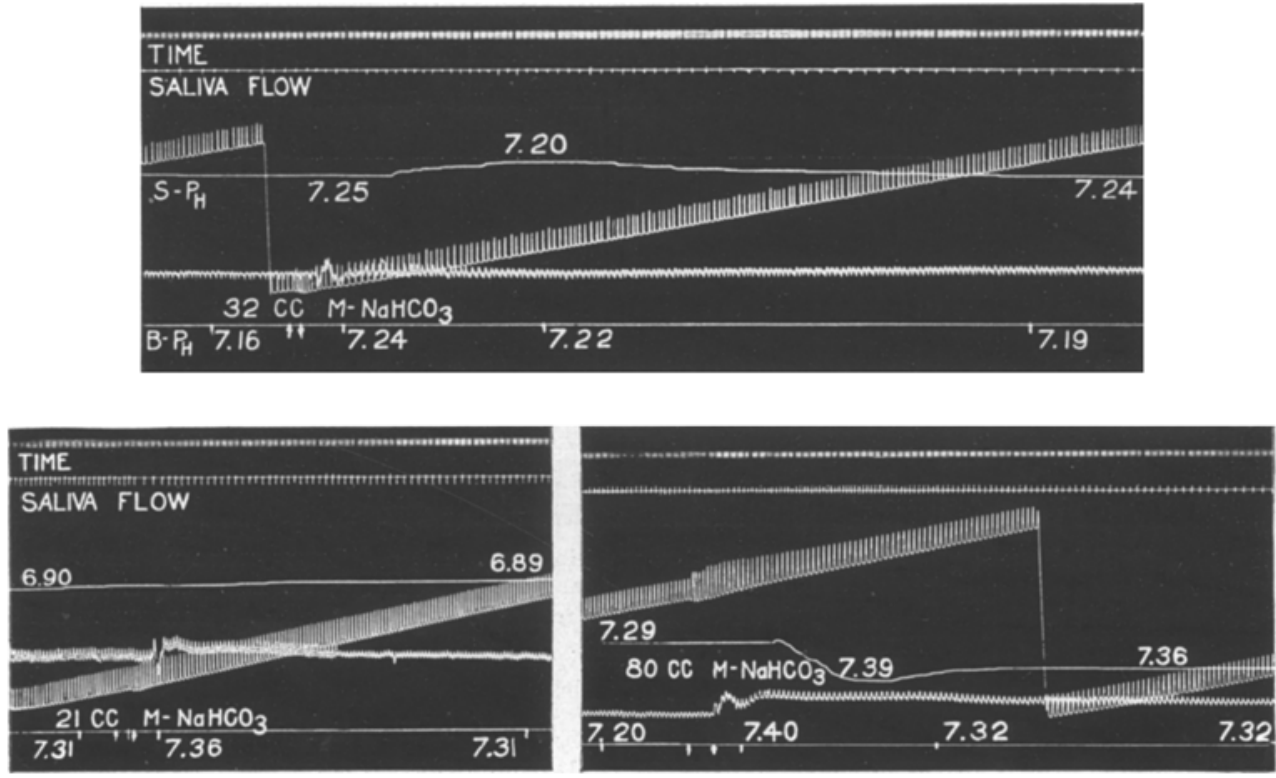

Fig. 146 to 148 . Intravenous injection of sodium bicarbonate increases saliva $c_{H}$ in fig. 146 , has no effect in fig. 147 and decreases it in fig. 148. These results agree with other findings on sodium bicarbonate. see text. [Brassfield and Bemrman: Amer. J. Physiol. 122, 21 (1938).]

be regarded as the probable stimulus. Henderson and Greminerg (158) attempted such experiments in dogs poisoned by intravenous injection but failed to obtain convincing results. WINDER (159) tried to overcome objection 
to their procedure by localizing the action of monoiodoacetic acid to the vascularly isolated carotid body. Among the numerous difficulties of his experiments was the gauging of the dose of monoiodoactic acid sufficient to prevent glycolysis without producing undue depression. To quote

" Unfortunately, in this series there were five out of seven experiments in which both the anoxic and hypercapnic, and also the asphyxic responses were abolished. In the remaining two experiments conditions were somewhat more, but not ideally, favorable for differential abolishment of the anoxic response. At stages of these two experiments where the normal anoxic stimulation was gone, the hypercapnic response persisted, though to be sure considerably weakened. More important, carbon dioxyde was definitely able to stimulate under ex. treme anoxia (asphyxial fluid), which by itself was unable to stimulate. This is considered evidence that glycolysis is an actual link in setting up the local excitatory process by anoxia, rather than merely a necessary source of energy during the short period of anoxia."

Bernthal (152), in his studies on the nature of the activating stimulus of the carotid body not only established to his satisfaction a correlation between intracellular acidity and carotid body activity associated with experimental variations in the bicarbonate system but also found, in agreement with theory, that local oxygen want or local cyanide poisoning produced a simultaneous vascular constriction and augmented pulmonary ventilation. He also demonstrated subnormal
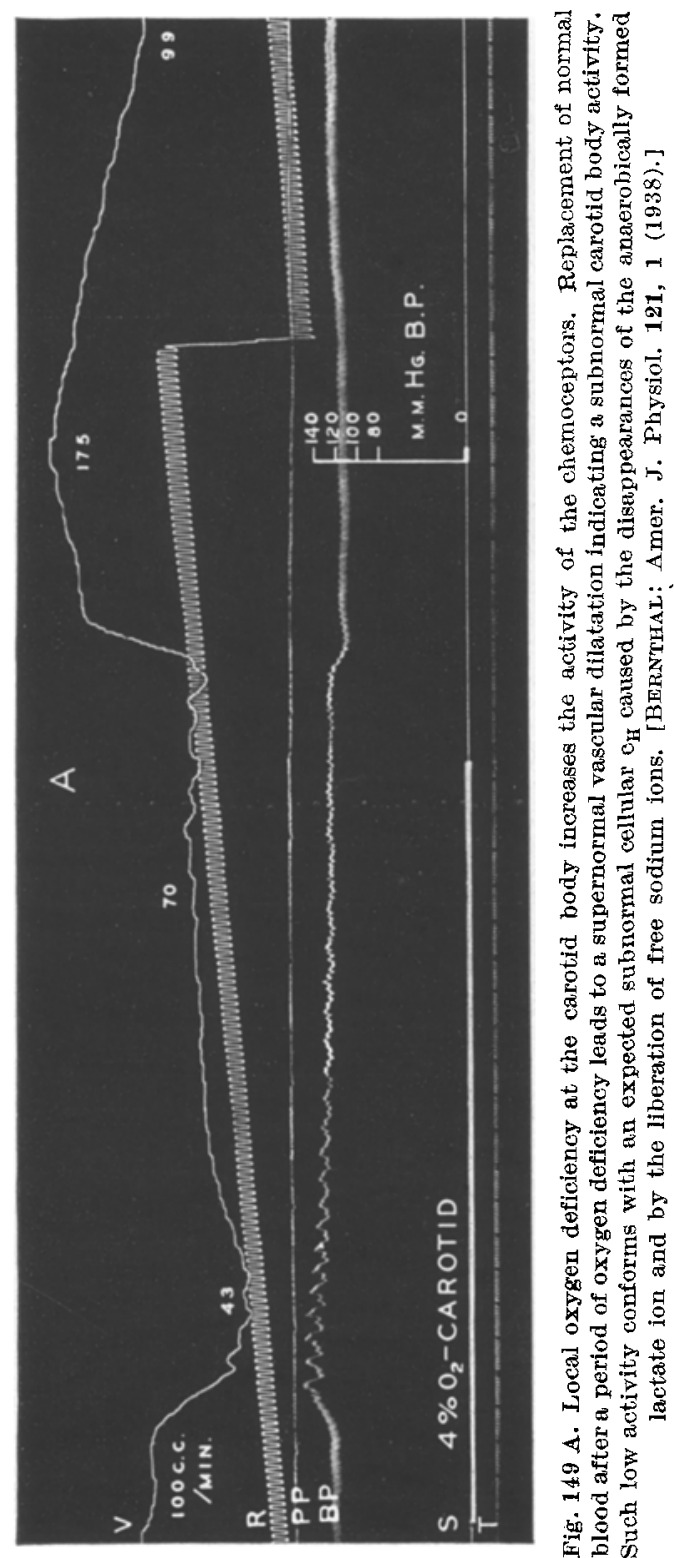
breathing on resupplying the carotid body with its normal quota of oxygen following a period of oxygen want (see fig. $149 \mathrm{~A}$ for vascular effects). This subnormal chemoceptor activity conforms with the original theories of MEYERHoF and Hru. As the lactate ion disappears free sodium is liberated which combines with carbon dioxide. This reaction turms the chemoceptors more alkaline and holds their activity in obeyance 
until their carbon dioxide content has returned again to normal. BernthaL concludes as follows

"Using reflex vasoconstriction and dilatation as indices respectively of increased and decreased chemoreceptor discharge, a close correspondence was demonstrated between changes in chemoceptor activity and theoretically co-existent changes in chemoceptor acidity. Thus the principles established by GEsEIT. in his theory of the chemical (acid) control of respiration and circulation may be applied to the carotid body as well as to the intra-cranial portions of the chemically sensitive control mechanism."

In the opening statement of Winder, Bernthal and WeEks (160) on Reflex Hyperpnea and Vasoconstriction due to Ischenico Excitation of the

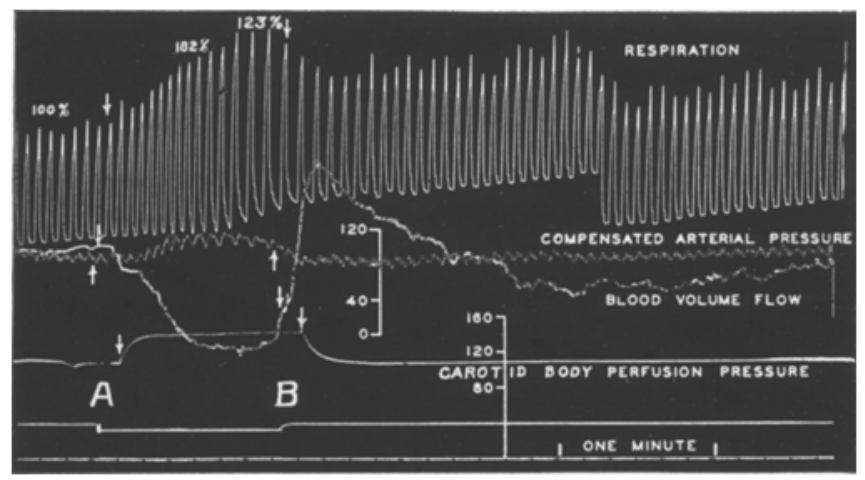

Fig. 149 B. Local ischemia of the vascularly isolated carotid body intensifies the activity of the chemoceptors as shown by increased pulmonary ventilation and the decreased rolume flow of blood or increased vascular constriction. Deocclusion of the carotid artery leads to a return of normal activity. The temporary overshooting of the volume flow of blood is probably due to the excessive washing out of carbon dioxide from the vasoconstrictor center during the preceding period of hyperpnea.

Carotid Body attention is called to a prediction in STARLINGs Physiology (1933) "that an extension of Geseurs (1925-1926) theory of chemical control of respiration to the recently discovered carotid chemoceptors would probably still explain known facts." They add

"The extension is receiving a growing body of factual support. The epithelioid cells of the carotid body, on anatomical bases presumably the specific receptor cells (DE CASTRO, 1927-1928), have an internal structure suggestive of an active metabolism (DE CASTRo, 1926). Since they have a relatively high volume to surface ratio, they may be more susceptible than the common cell to an inadequate environment in relation to their own metabolism."

Winder, Bernthal and Weeks reason

"If, as such evidence indicates, the state of excitation of the chemoreceptors is a product of interaction of their own acid metabolism with their immediate environment rather than primarily a function of the chemical quality of the environment per se, then failure of refreshment of the environment should lead to a cumulative disturbance resulting in excitation. That is, a purely quantitative anemia of the chemoreceptors without exogenous change in the quality of the blood should reflexly excite respiration and cardiovascular changes. The present experiments test whether this additional requirement of the hypothesis is to be satisfied."

Reference to fig. 149 B shows increased breathing and vascular constrietion during temporary ischemia brought about by occlusion of the carotid artery between $\mathrm{A}$ and $\mathrm{B}$ and recovery with overshootings on deocclusion of the 
carotid arteries at B. Pronounced apnea on deocclusion of the carotid arteries after a prolonged ischemic hyperpnea may also occur (fig. 150). When breathing is again resumed periodicity often occurs just as it does during recovery from anoxemia in the intact animal.

Instead of taxing the respiratory exchange of the chemoceptors of the carotid body by restricting the blood supply, the procedure may be changed

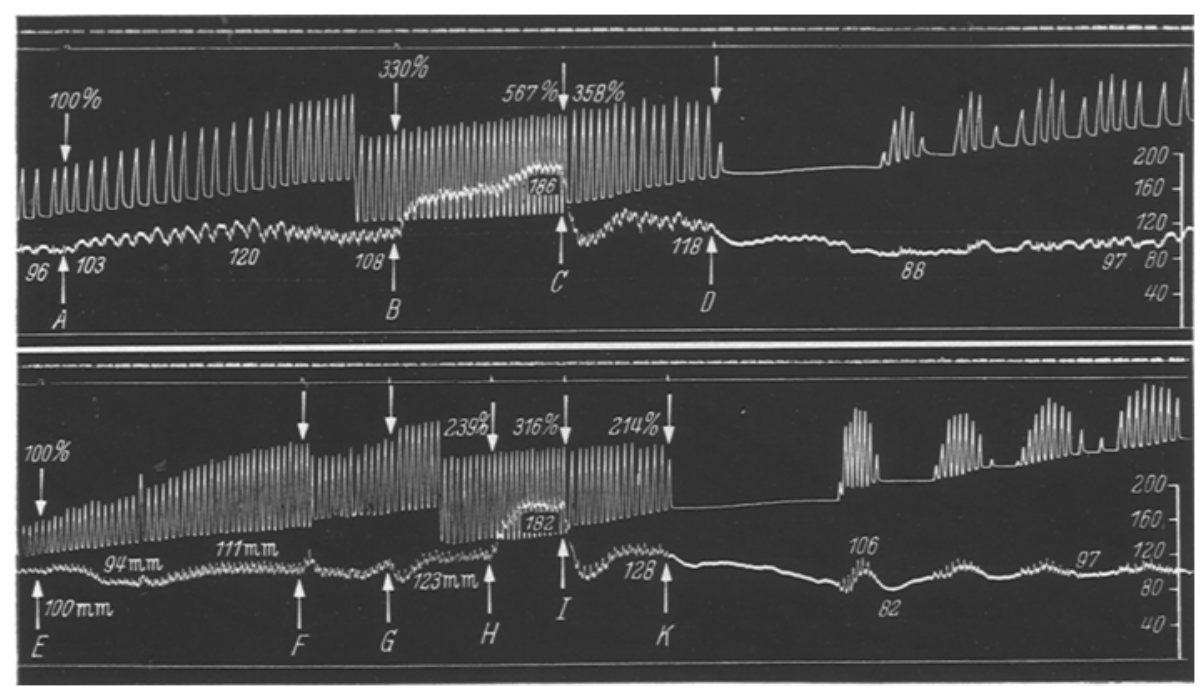

Fig. 150. Local ischemia of the carotid body produces the same intense hyperpnea as noted in fig. $149 \mathrm{~B}$. Arterial occlusion was started at $A$ and $E$ in the upper and lower records and terminated at $D$ and $K$. The intermediate procedure may be disregarded. Termination of ischemia will be seen to produce a long apnea followed by periodic breathing. The apnea is attributable to the preceding hyperventilation and its consequent central hypocapnia. These results are significant in their resemblance to those of hypooxic hyperpnea in which the increased acidity and activity of the chemoceptors also lowers the $c_{H}$ of the remainder of the body. [Winder, Bersthal and Weeks: Amer. J. Physiol. 124, 238 (1938).]

by simply increasing the metabolic demands, leaving the circulation unchanged. With that in mind Bernthas and WeEks (161) altered the temperature of the carotid body, perfusing it with blood at different temperatures, and recorded activity changes in terms of pulmonary ventilation and vasoconstriction. Cooling the glomus, in fig. 151, from $37^{\circ} \mathrm{C}$ to $15^{\circ} \mathrm{C}$ decreased the amplitude and frequency of breathing (see also fig. 152). Increasing the temperature above normal, to $43^{\circ} \mathrm{C}$, produced superventilation.

Glomus activity, as indicated by vasoconstriction, must be studied during uniform ventilation due to the interfering action of central hypocapnia. Fig. 153 shows the diminution of activity under such conditions produced by a lowered glomus temperature. Bernthal and WeExs conclude that their "findings demonstrate a further correspondence between changes in chemoceptor aetivity and theoretically concurrent changes in chemoceptor acidity and they are therefore inclined to support the hypothesis that the functional activity of chemoceptors is dependent upon the rate and 
quality of their own acid metabolism as well as upon the properties of their environment."

It is difficult to follow the arguments of Schmid and Comroe (171) on the cellular acidity theory of chemoceptor activity in their recent review
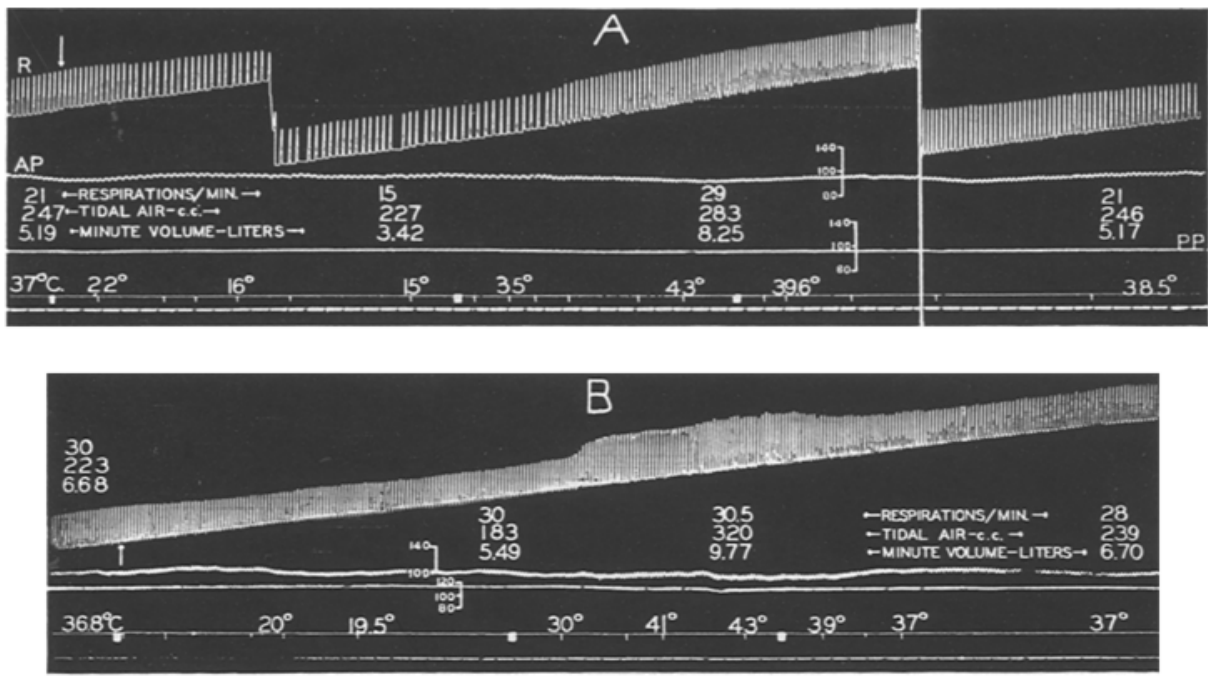

Fig. 151 and 152. Pulmonary ventilation is diminished by circulating blood at subnormal temperatures through the rascularly isolated carotid body. On increasing the temperature of the blood above normal. breathjng is also increased to supernormal values. Blood temperatures, freqnency of breathing, tidal air and minute volume of breathing are indicated on the records. The fact that cooling of the chemoceptors decreases breathing leads to the conclusion that they are tonically active under normal conditions of blood flow [Bernthal and Weeks: Amer. J. Physiol. 127, 94 (1939).]

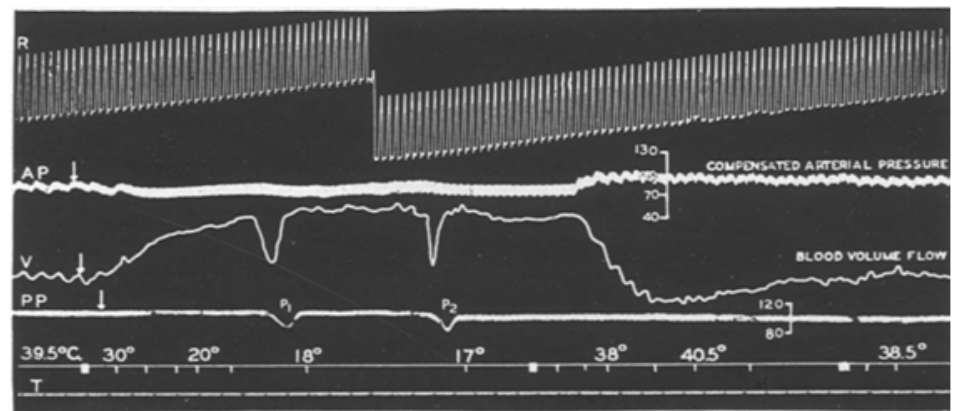

Fig. 133 . Cooling of the carotid body decreases the normal activity of the chemoceptors as indicated by the jnereased volume flow of blood or vascular relaxation. The dips in the volume flow curve are experimental artefracts and may be disregarded. Pulmonary ventilation is uniform. [BerNTHAL and Wenks: Amer. J. Physiol. 127, 94 (1939).]

on the "Function of the Carotid and Aortic Bodies." Factual evidence, pro or con, such as that coming from the distant laboratories of the Karolinska Institut at Stockholm seem to us far more significant. I refer to the experiments of von EuLER, Liljestrand and ZotTerman (162) on the activity of the carotid bodies registered electrically in HenINGs nerve. They describe a high sensitivity to changes in oxygen and carbon dioxide pressures diverging 
from normal and a linear relation of activity to the pressures of these gases, direct for $\mathrm{CO}_{2}$ and inverse for $\mathrm{O}_{2}$ (see fig. 154 and 155). $\mathrm{NH}_{4} \mathrm{OH}$ abolishes the activity of the carotid body during oxygen deficiency, but has no effect upon the stimulating action of nicotine and lobeline, so these drugs are thought to act central to the point at which oxygen lack and carbon dioxide stimulate. Reasons are given for assuming that these alkaloids act on the carotid body in a way analogous to their action on sympathetic ganglia. The authors con-

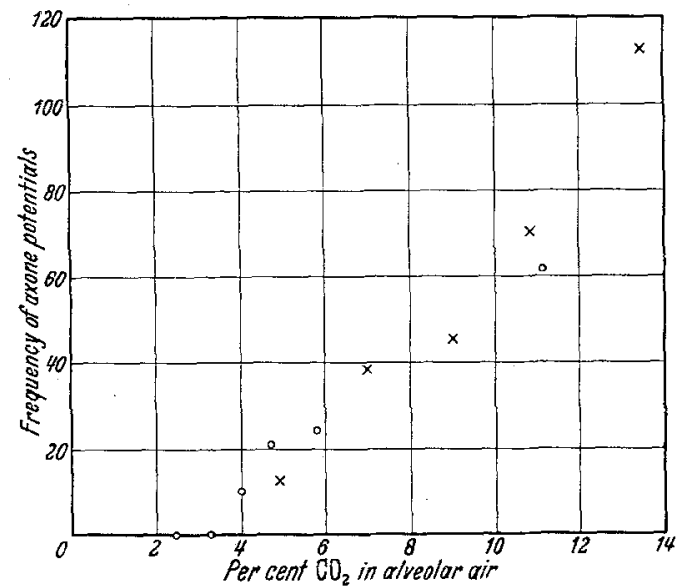

Fig. 154. clude that their observations "speak strongly for the interpretation that

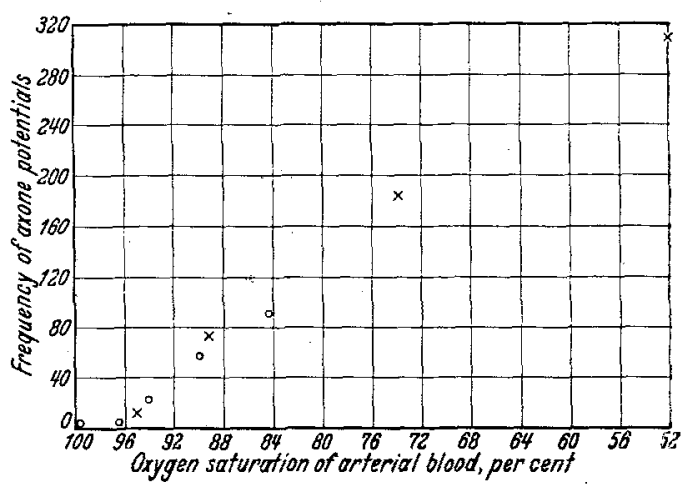

Fig. 155.

Fig. 154 and 155. Graphs showing the relation of the frequency of potentials in nerve fibers of HERINGs nerve to the oxygen saturation of the blood and to the percent of carbon dioxide in the alveolar air. [Etler von, LilJestrand and ZotTerman: Skand. Arch. Physiol. (Berl. u. Lpz.) 83, 132 (1939).]

both kinds of stimuli $\left(\mathrm{O}_{2}\right.$ lack and $\mathrm{CO}_{2}$ excess $)$ act by increasing the $\mathrm{c}_{\mathrm{H}}$ in the chemoceptors."

In the original development of the cellular acidity theory Geselu considered the additive effects of oxygen lack and carbon dioxide excess as a strong argument favoring the theory, for if the effects of oxygen lack are due to acid, the superposition of a second acid effect by the administration of carbon dioxide would perforce increase the original stimulation. GELLHORN and his associates (163) agree to the greater combined action but are not prepared to interpret this effect in terms of acid. They are also perturbed by the fact that the brain turns more alkaline than normal during oxygen deficiency and employ that argument against the cellular reaction theory of respiratory control. But if it be remembered that the cellular acidity theory actually insists upon an hypocapnic condition of the brain their argument no longer holds. In addition they are concerned about the failure of lactic acid to accumulate in the blood despite prolonged exposure to high altitudes [Dill, Talbott and Consolario (164)] but it must be recalled that a dearth of lactic acid in the blood stream at low oxygen pressures could be regarded as evidence for the perfection with which the theory is actually working. The limitation of anoxic stimulation to the chemoceptors, in our opinion, makes 
the concept of additive action of oxygen lack and carbon dioxide excess a most simple one.

\section{The Interaction of Central and Peripheral Chemical Control of Breathing.}

Before the important discoveries by Heymans (149) of the respiratory function of the carotid body it seemed necessary to consider only one chemically sensitive regulatory structure in the explanation of the chemical control of breathing. Since then the problem has taken on new aspects and new relations which have engaged the interests of many. Important among these problems still under investigation are the relative importance of the central and peripheral chemical control, the relative sensitivities of the center and of the chemoceptors to carbon dioxide, the relative sensitivities of the center and of the chemoceptors to oxygen, and the interaction between the central and peripheral controls.

A determination of the relative importance of central and peripheral chemical control of breathing is no easy matter. When Cromer and Ivy (166) studied dogs, trained to work on the tread mill, they found them apparently as efficient after denervation of the sinus regions as before, and DAUTREBANDE (167, 168) maintains that dogs, deprived of their peripheral chemoceptor support, apparently acclimate and adjust their breathing to high altitudes in a manner similar to that of the complete dog. Strong difference of opinion exists on the latter point, but even granting the validity of DaUTREBandes findings it seems precarious to conclude that the role of the chemoceptors is unimportant. A compensation for a lost function does not necessarily mean that the lost function is of relatively little value.

If sensitivity of control is helpful to the general well-being of the organism, statements concerning the unimportance of the reflexogenic control of breathing should be considered with great care. The increased action potentials of Herings nerve which are evoked by minor changes of carbon dioxide pressure within the physiological range [SAMaAN and Stelua (169)], the elevation of alveolar carbon dioxide by the denervation of the carotid bodies [VoN EULER, LilJestrand and ZotTERMaN (162)], the increased activity of the isolated carotid body (measured in terms of vasoconstriction and pulmonary ventilation) by small changes of oxygen and carbon dioxide pressures [BERNTHAL (152)], the increased activity resulting from small changes of temperature [Bernthal and WeErs (161)], the increased electrical activity of the sinus nerve to an oxygen desaturation of blood below $96 \%$ or to an increased alveolar carbon dioxide pressure above $30 \mathrm{~mm}$. Hg [yon Euler, Luldestrand and ZotTerman (162)], all speak for a nicety of regulation by the chemoceptors. Observations, such as these, could readily dismiss statements so commonly 
found in our standard textbooks of physiology that a reaction to oxygen deficiency does not set in until the oxygen percentage of the inspired air has fallen to 12 .

There can be no question whatever concerning the high sensitivity of the carotid body to oxygen deficiency as compared with the respiratory center. In the anaesthetized animal, at least, it is very much greater. Yet it cannot be held that the center is completely incapable of responding to either lack of oxygen or to the administration of cyanide. The threshold of stimulation to be sure is much higher, the incidence of stimulation to depression is low, but the observations of positive stimulation are too numerous to be ignored. Nor is this meant as an argument supporting a central stimulating action of oxygen lack in hypooxic hyperpnea. On the contrary, the center is regarded to be chemically apneic (hypocapnic) during intense hyperpnea of such origin. Nevertheless it must be realized that if oxygen lack exerts a central excitatory as well as a depressant action, in chemoceptively denervated animals, it must be capable of contributing such action at any time that oxidations are impaired. For that reason it seems advisable to retain the excitatory as well as the depressant potentialities of oxygen deficiency under all conditions. If of no other value, such central excitatory action as is demonstrable gives support to the cellular acidity theory according to which the activity of the carotid body may be evoked by oxygen deficiency. For further accounts on the relative sensitivity and importance of the central and peripheral mechanisms several other recent reviews are available [Heymans (149), Geseld (147), Schmidt and Comroe (171), Johnson, Forbes, Dilu and Henderson (172)].

Closely related to the question of relative sensitivities is the problem of joint action and interaction of the two chemical mechanisms of control. Recent studies of this phase of the problem [Geseli, Lapides and Levin (173)] have revealed some unsuspected relationships which seem to clarify certain aspects of the chemical control of breathing. While the results to be described are by no means as refined or as quantitative as many already described above, they have the advantage of simplicity and of revealing underlying principles missed by the more fundamental approaches in the individually isolated systems working by themselves.

Both vagi were cut to eliminate aortic presso and chemoceptor influences. Effective cold blocking devices, chilled or warmed with circulating alcohol, were kept in place on both sinus nerves. The sinuses were permanently collapsed to eliminate pressoreceptor effects. Blocking and deblocking Herings nerves thus served to abolish and reestablish at will all theoretically existing chemoceptive stimulation and allowed comparison of breathing of purely centrogenic chemical origin with breathing of combined centrogenic and reflexogenic origin. Such comparisons were made under several important modifications of respiratory conditions. 
The first experiments (fig. 156) show the role of the chemoceptors in cutting short the duration of apnea produced by standard artificial overventilation with room air. The periods of overventilation employed to produce hypocapnia and the periods of sinus nerve block are indicated by the horizontal bars. In fig. $156 \mathrm{~A}$ artificial overventilation producing an apnea of approximately one minute when the carotid bodies are out of function, is insufficient to produce apnea of any duration when the chemoceptors are normally functioning again. Fig. $156 \mathrm{C}$, in which Herings nerves are functioning, shows that the signals arising in the carotid bodies cut apnea from approximately 60 seconds (see fig. $156 \mathrm{~B}$ where the nerves are blocked), down to 20 seconds. Certainly this is a most striking effect. The chemoceptors are most decidely effective in this capacity of avoiding long pauses in breathing, particularly when this action is compared with the relatively small depression of breathing noted on blocking Herings nerve during eupnea (fig. $156 \mathrm{C}$ ). The chemical stimulation at the carotid bodies which assists the center in reinstating breathing is no doubt a combination of carbon dioxide excess. and oxygen lack of which the second is probably the most important.

The following observations concern carbon dioxide. Much evidence, not as quantitative as might be desired, has accumulated indicating that the center is as sensitive, if not more so, as the chemoceptors to carbon dioxide. If this is true, the central mechanism should respond to a smaller rise of carbon dioxide pressure than the chemoceptors. But even so, breathing still remains a resultant of the centrogenic and peripheral or reflexogenic action of carbon dioxide. (Refer back to fig. 22 which shows how the reflexogenic electrotonic current adds on to the centrogenic electrotonic current and increases the frequency of discharge.) A reduction of breathing such as occurs when Herings nerves are blocked, therefore, does not mean that the chemoceptors are more sensitive than the center to carbon dioxide. The same directional change should occur regardless of the relative sensitivities. According to our views, the results simply indicate that the reflexogenic component has been withdrawn from the centrogenic component upon which it builds.

To analyze the joint action of central and reflex response to carbon dioxide, dogs were allowed to rebreathe into a restricted volume of gas containing $40 \%$ oxygen. The high percentage of oxygen was intended to confine the response to the increasing carbon dioxide percentages which follow on rebreathing. It will be seen (fig. 157) that Herist nerve block produced an appreciable reduction in breathing so long as the carbon dioxide percentage of the inspired air was low $(1.5$ and $3.5 \%)$. At $5 \%$ the reduction was exceedingly small and at $6 \%$ it seemed entirely missing. Breathing however continues to increase with increasing hypercapnia indicating that the hyperpnea of the higher intensities is entirely centrogenic. The relative intensities of centrogenic and reflexogenic breathing of hypercapnic origin are indicated in the second 
and third schemas of fig. 159 . The solid black area J, K, H, I, L of the second schema shows the increasing volume of centrogenic hypercapnic breathing
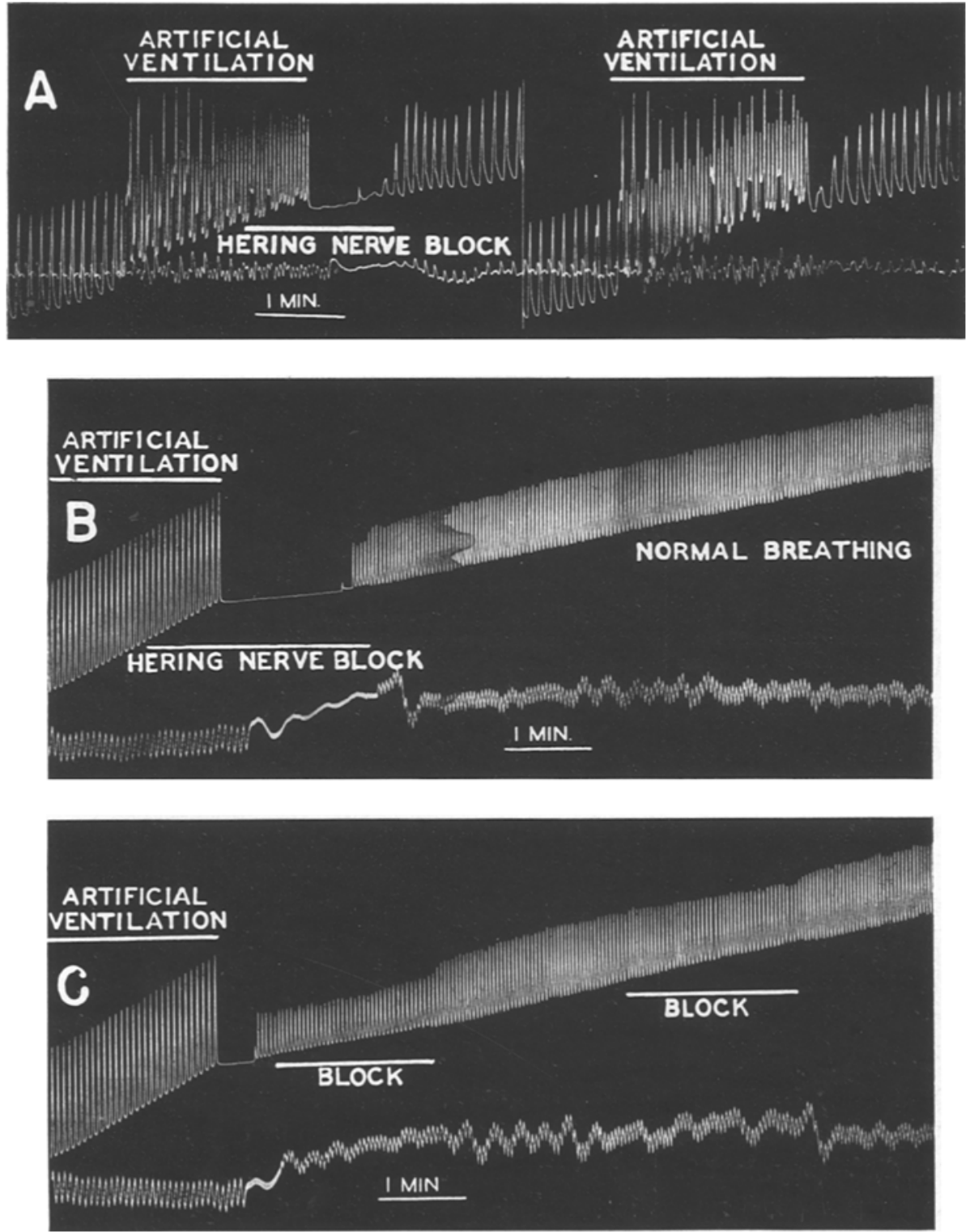

Fig. 156. The influence of the carotid bodies on the duration of apnea produced by artificial overventilation of the lungs after double vagotomy. The duration of apnea is much longer when HERINGs nerves are blocked indicating a high sensitivity of the chemoceptors to changes in oxygen and carbon dioxide pressures within the physiological range. The two observations in the upper record are to be compared with each other, and record $B$ is to be compared with record C. For further details see text. (Gesell, LAPIDEs and Levin: Amer. J. Physiol. In Press.)

and the cross hatched area $G, H, K$ shows the diminishing volume of reflexogenic breathing with increasing hypercapnia. On the basis of the findings of 
von Euler, Lulusastrand and Zotterman (162) demonstrating a linear relation of chemoceptor activity to concentration of carbon dioxide, we have assumed that the actual activity of the carotid bodies is represented by our cross hatched area $\mathrm{M}, \mathrm{Q}, \mathrm{N}, \mathrm{O}, \mathrm{P}$ of the lower figure. Consequently, if breathing is a resultant of centrogenic plus reflexogenic activity, if reflexogenic activity is a linear function of concentration of carbon dioxide, and if Herivg nerve block produces no reduction in breathing, it follows that the effects of chemoceptor activity must be abolished by carbon dioxide at some point central to the carotid bodies. We have seen how respiratory reflexes are weakened by the central action of carbon dioxide (see section 28). It is, therefore, suggested that carbon dioxide not only sets up chemoceptor reflexes in the periphery

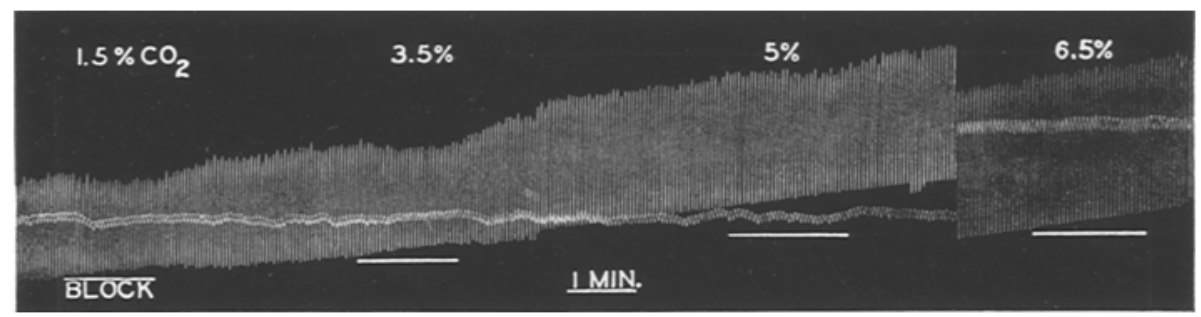

Fig. 157. Repeated cold block of HERINGs nerve during progressive hypercapnic byperpnea show decreasing reflexogenic breathing. When the inspired air contained approximately $6.5 \%$ carbon dioxide HERING nerve block produced no reduction in breathing, indicating that hyperpnea at sthis concentration is purely centrogenie (Grsec, Tapides and LFvis: Amer. J. Physiol. In Press.)

but at the same time weakens these very same reflexes by virtue of its central action. As reflexogenic activity is abolished centrogenic activity of increasing proportions steps in to take its place.

The picture is very different with increasing hyperpnea produced by an unbroken progressive reduction of oxygen in the inspired air (fig. 158). The dog is connected again with a rebreathing tank, containing at the very beginning a $40 \%$ oxygen mixture in nitrogen. Under these conditions HERING nerve block produces a slight reduction of breathing, explainable by the elimination of the peripheral stimulating action of carbon dioxide. Similar cold block with the oxygen of the inspired air at $19.7 \%$ produces a decidedly greater reduction of breathing thus indicating the highly sensitive response of the chemoceptors to oxygen deficiency. At $16.6 \% \mathrm{O}_{2}$, breathing is cut still more, not only below that of the hypooxic hyperpnea but distinctly below that of the eupnea when the oxygen stood of $40 \%$. This shows the graded response of chemoceptor activity to graded oxygen deficiency. At $12.5 \% \mathrm{O}_{2}$, breathing is reduced still more. In fact there is a momentary cassation which gives way to a periodic type of breathing. At $8.6 \%$, removal of chemoceptor support leads to a prolonged apnea, which only after a period of approximately two minutes gives way to centrogenic hyperpnea. This shows that the chemoceptors take complete charge of the chemical control of breathing even at relatively high oxygen 

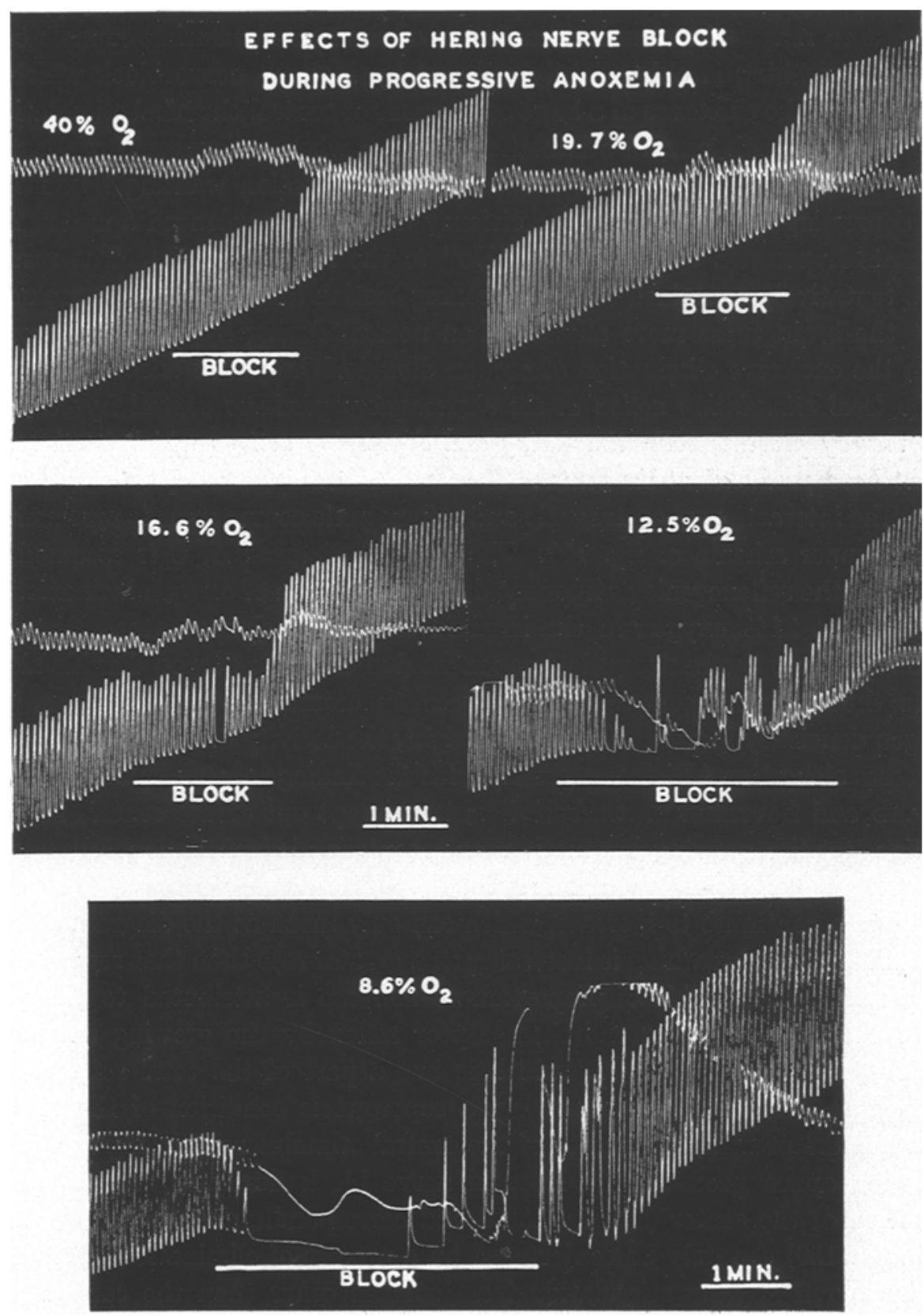

Fig. 158. Repeated HERING nerve block during progressive hypooxic hyperpnea in a vagotomized dog. The increasing reduction of breathing which occurs with each cold block indicates the importance of reflexogenic breathing during hypooxia. The occurrence of apnea when Herings nerves are blocked at $8.6 \% \mathrm{O}_{2}$ shows that breathing may become purely reflexogenic at relatively high oxygen pressures. The fact that breathing is markedly depressed. when the chemoceptor signals are abolished at $19.7 \% O_{2}$ in the inspired air shows the sensitive response of the chemoceptors in the physiological range of oxygen pressure. [Gesell, Lapides and Levin: Amer'. J. Physiol. In Press.) 
pressures. Now this apnea is a most important phenomenon because it is thought to reflect the chemical condition of the center. During eupneic conditions, breathing is only slightly reduced by Hearsa nerve block which is evidence for the existence of a large component of central chemical stimulation. Why then is such central stimulation missing when reflexogenic support is withdrawn during hypooxic hyperpnea? Is stimulation wanting because of the depressing action of oxygen deficiency during the prolonged apnea? The altimate hyperpnea would answer "No" to the latter question. The results were tentatively explained in the preceding section in which it was concluded that the brain turns more alkaline then normal during hypooxic hyperpnea from the excessive elimination of carbon dioxide. Oxygen deficiency by virtue of its local stimulating action at the chemoceptors produces central hypocapnia and thereby prevents both stimulating and depressing actions which it otherwise might have produced at the center.

The interaction of centrogenic and reflexogenic components is represented in the upper schema of fig. 159. The vertical distance $A, C$ is assumed to represent the reflexogenic component due to the action of oxygen lack existing normally at $21 \%$ oxygen in the inspired air at the chemoceptor. Distance $\mathrm{C}, \mathrm{E}$ represents the centrogenic component due either to carbon dioxide alone or to the combined central effects of carbon dioxide and oxygen lack. As oxygen deficiency increases from left to right, the activity of the carotid bodies increases along line $\mathrm{AB}$, in conformity with the findings of voN EuLER. LILJEstrand and Zotmerman. Carbon dioxide is swept out of the centers in proportion to the growing ventilation and centrogenic ventilation falls along line CD, in conformity to the growing hypocapnia. Nevertheless the total ventilation continues to increase, which can only mean as the diagram shows, that reflexogenic breathing is increasing at a greater rate than that represented by gradient A, B. This disproportionate increase is attributed to the progressive augmentation of the chemoceptor reflex by the growing alkalinity of the center. Triangle $\mathrm{DFD}^{\prime}$ which is but a part of the total reflexogenic component $\mathrm{ABD}^{\prime} \mathrm{C}$ simply represents a superapneic condition of the center.

For the present, the nature of the central stimulus reinitiating breathing following apnea produced by HenING nerve block during a "super apneic condition of the center (see fig. $158 \mathrm{C}$ and corresponding conditions at $8.6 \%$ $\mathrm{O}_{2}$ in fig. 159) can only be conjectured by a process of elimination. Provided unknown reflexogenic stimulation from sources other than the carotid and aortic bodies can be disregarded, the reinitiated breathing must be considered of centrogenic origin. Had the apnea been caused entirely by a paralyzing action, that action would have been expected to increase and to have terminated in death. Had the stimulation of breathing been one of direct action of $\mathrm{O}_{2}$ lack, there should have been supernormal rather than subnormal centrogenic breathing when Hering nerve block took effect. But if the apnea was due 
to acapnia, time was essential for a reaccumulation of acid and a rebuilding of the central stimulus. We suggest that this occurred partly as a result of
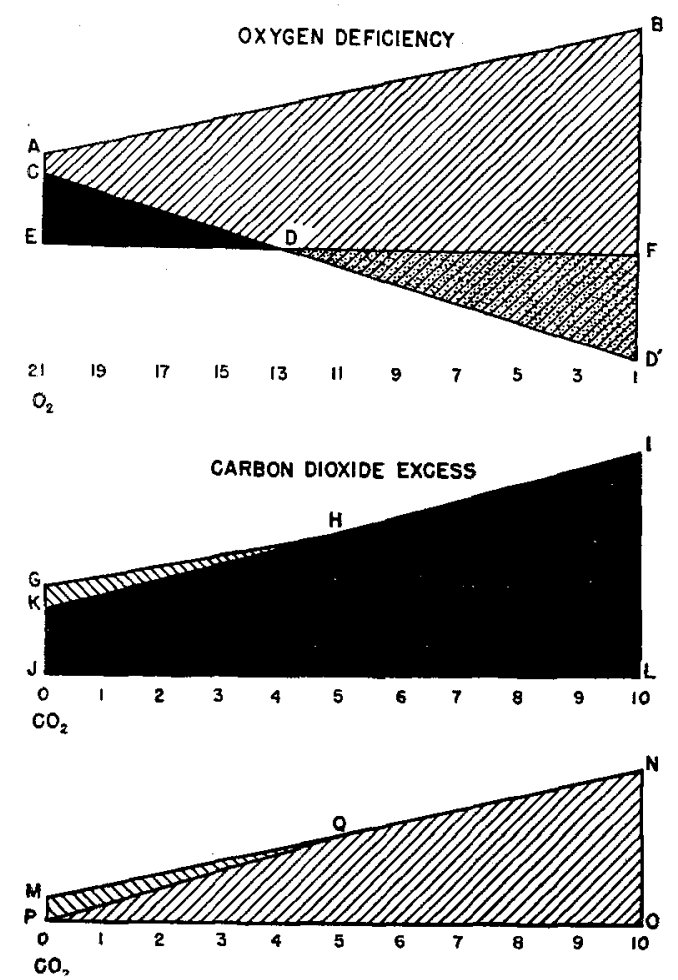

Fig. 159. A schematic representation of the changing proportions of centrogenic and reflexogenic breathing during progressive hypooxic and hy percapnic breathing. The relative intensities of centrogenic and reflexogenic breathing were determined by temporary bilateral blocking of HERINGs nerves in vagotomized dogs. Area $\mathrm{A}, \mathrm{B}, \mathrm{D}^{\prime}, \mathrm{D}, \mathrm{E}$ illustrates results obtained during progressive oxygen deficiency and areas $G, I$, $\mathrm{L}, \mathbf{J}$ and $\mathbf{M}, \mathrm{Q}, \mathrm{N}, \mathrm{O}, \mathrm{P}$ show findings during progressive hypercapnia. The solid black areas indicate the amount of centrogenic breathing and the cross hatched areas indicate the simultaneous degree of reflexogenic breathing. During oxygen deficiency centrogenic breathing diminishes with a decreasing amount of oxygen in the inspired air. Apnea comes on when HERINGs nerves are blocked with the per. centage of oxygen at approximately 13. (See point D.) The duration of apnea grows as the degrees o oxygen deficiency increases which suggests a progressively increasing hypocapnia of the center. The augmenting hypocapnia is represented by the stippled cross hatched area $\mathbf{D}, \mathbf{F}, \mathbf{D}^{\prime}$. It is apparent that reflexogenic breathing increases as a result of two changes: an augmenting chemoceptor activity and a decreasing centrogenic breathing. From point D reflexogenic breathing grows despite an apparently opposing hypocapnic condition of the center. A more logical interpretation seems to be that it increases through the aid of the intensification of reflexes resulting from an increasing alkalinity of the centers. During hypercapnia the proportions of centrogenic and reflexogenic breathing change in opposite directions. Reflexogenio breathing disappears with the carbon dioxide of the inspired air at approximately $6 \%$, while centrogenic breathing continues to increase. With chemoceptor activity inereasing along line $M, N$ area $M, Q, N, O, P$ represents the potential amount of peripheral chemical stimulation. of this amount of potential stimulation set up in the peripheral chemoceptors $P, N, O, P$ is abolished by the central hypercapnic action. The most obvious and striking conclusion reached on comparing the results of hypooxia and hypercapnia is that breathing tends to become purely reflexogenic during oxygen deficiency and purely centrogenic during hypercapnia. (Geseld, Lapides and Levin: Amer. J. Physiol. In Press.)

the high anaerobic acid metabolism in the brain [McGinty and Geseul (166)] and partly as an effect of the reaccumulating acid in the blood.

For completeness it must be mentioned that apneas frequently did terminate in death without outward signs of respiratory stimulation. It is, therefore, 
reasonable to assume that depression capable of completely counteracting stimulation can and does occur. Signs of such depression are visible in the falling blood pressure during the last two nerve blocks of figure 158 .

These experiments, we believe, support the evidence which is accumulating in favor of an all inclusive cellular acidity theory of respiratory control, for the center and for the chemoceptors alike. The peculiar interaction between chemoceptor and central activity offers such unified interpretation, e. g. the supernormal alkalinity of the brain can no longer be used as an objection to the acceptance of the cellular reaction theory. The acidity theory demands that such conditions should and do prevail for the well-being of the organism as a whole.

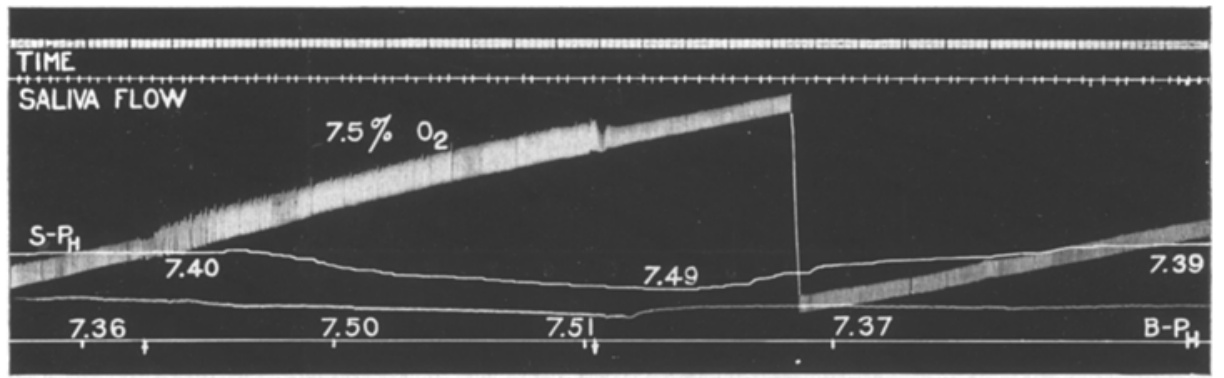

Fig. 160. The administration of a gaseous mixture low in oxygen decreases the hydrogen ion concentration of the saliva and arterial blood when compensatory pulmonary rentilation is allowed to occur. Changing $c_{H}$ is recorded with a glass electrode in the flow of saliva (see $S-P_{H}$ ). Blood $p_{H}$ determined on blood samples is indicated below (see $\mathrm{B} \cdot \mathrm{P}_{\mathrm{H}}$ ).

But Gellaorn and Lambert (163) maintain that the $c_{H}$ of the brain diminishes during the uniform artificial administration of a gaseous mixture low in oxygen. But what is meant by the $c_{\mathrm{H}}$ of the brain? Is it the $c_{\mathrm{H}}$ of the interior of a nerve cell, of the blood, or of the intervening tissue fluids? An electrode cannot at present be successfully placed within a brain cell and, therefore, direct measurements of intracellular $c_{H}$ are impossible. An electrode placed on the surface of the brain must be considered as lying outside the cell between the blood and the interior of the cell. It is, therefore, subjected to the dual influence of the blood and the cell. Actually the $c_{\mathrm{H}}$ of the blood diminishes during oxygen deficiency due to the reduction of the hemoglobin. The effect of that change should be to turn the electrode more alkaline. Theoretically the cell should turn acid as a result of the increased anaerobic metabolism. The effect of that change should be to turn the electrode more acid. The question then arises what will the resultant be and what does it signify?

The experiments of Brassfield and BerrrmanN on salivary $\mathrm{p}_{\mathrm{H}}$ indicated that the saliva, like the cerebrospinal fluid, like the chemoceptors and like the reflex centers, turned acid to the administration of carbon dioxide and sodium bicarbonate and alkaline to the injection of sodium carbonate. The electrode may, therefore, be regarded as figuratively within the salivary gland 
cells and possibly reflecting in general the $c_{\text {H }}$ changes occurring within cells during hypooxia. The alkaline change in the saliva during the administration

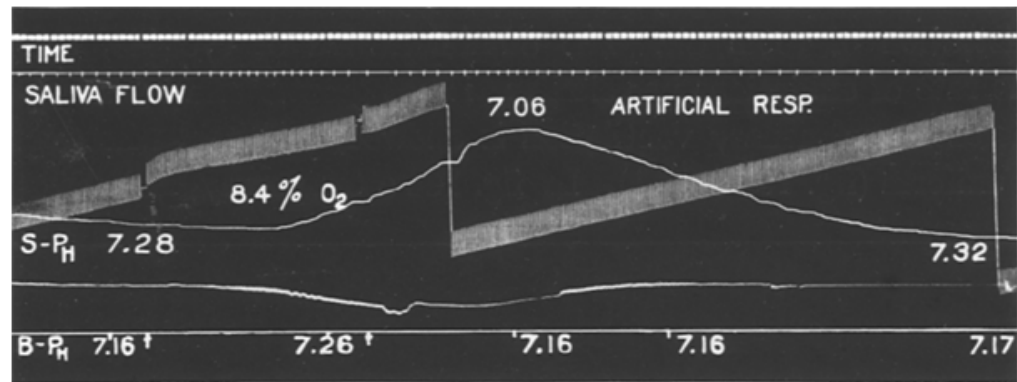

Fig. 161. When a gaseous mixture of even higher oxygen content is next administered by uniform artificial ventilation the saliva turned acid immediately despite a temporary decrease of blood $\mathrm{c}_{\mathrm{H}}$. It is, therefore, logical to conclude that oxygen lack tends to turn the glandular cells more acid than normal if the washing out of excess acid by increased pulmonary ventilation is prevented. [BRASSFIELD and Behrmann: Amer. J. Physiol. 122, 21 (1938).]

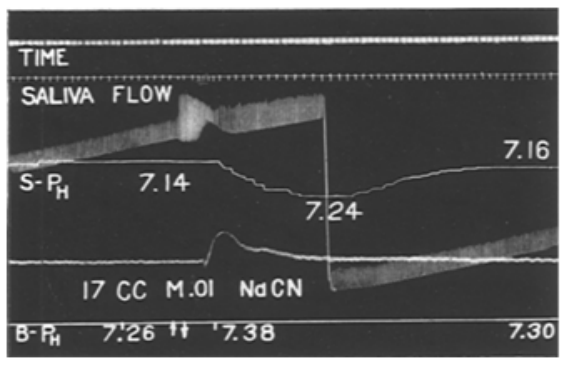

Fig. 162.

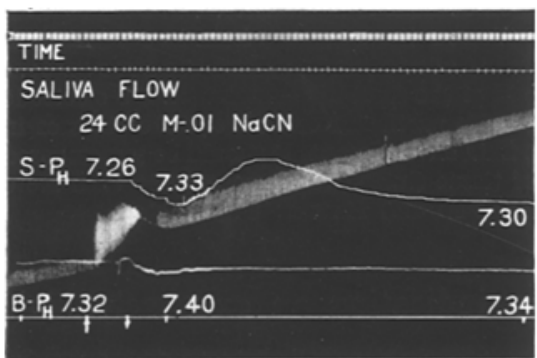

Fig. 164.

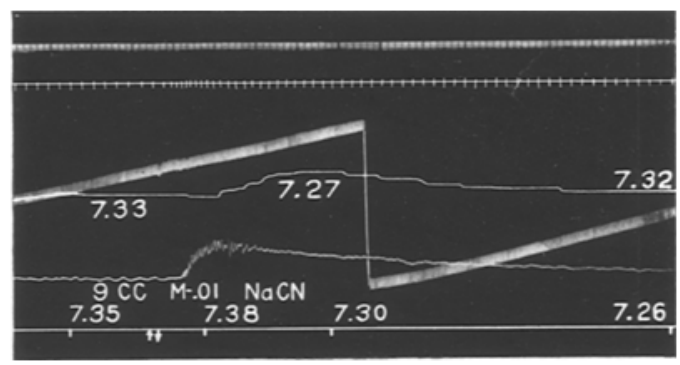

Fig. 163 .

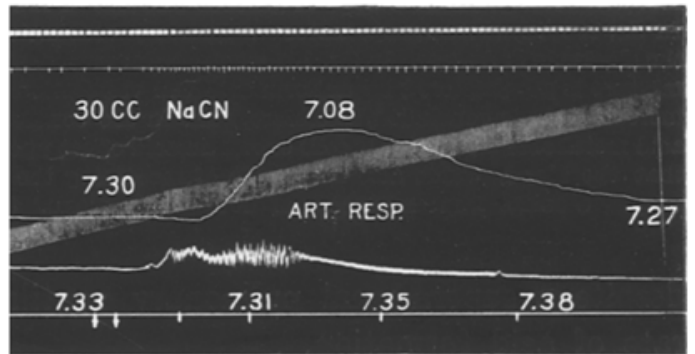

Fig. 165.

Fig. 162-165. When oxidations are impaired by the intravenous injection of cyanide the acidity of saliva and arterial blood change in a manner similar to that produced by oxygen deficiency. Compare the upper two fig. 162 and 163 and the lower two fig. 164 and 165 . When compensatory pulmonary ventilation was allowed in fig. 162 , both saliva and blood turned less acid. During uniform artificial pulmonary ventilation in fig. 163 both saliva and blood turned more acid. In fig. 164, in which a larger injection was given, and in which a greater anaerobic formation of acid must have occurred, the saliva first turns less acid and then more acid than normal. Eventually the augmented ventilation decreases salivary $c_{\mathrm{H}}$ below normal. When compensatory ventilation is not allowed the saliva turns highly acid for a long period of time. [Bragsmield and Behrmanv: Amer. J. Physiol. 1.22, 21 (1938).]

of a $7.5 \%$ oxygen mixture in fig. 160 in which the animal controls its breathing agrees with changes already described for blood and tissues. The hypocapnia produced by hyperventilation has turned the saliva alkaline. A slightly 
richer oxygen mixture administered in uniform volume, to avoid excessive elimination of carbon dioxide, leads to an opposite acid change (fig. 161). The saliva now turns distinctly acid. Recovery of normal values follow on the readministration of room air. The administration of cyanide, designed to lower the rate of oxidations has similar, if not greater effects, on $c_{\mathbb{H}^{-}}$-alkaline when increased pulmonary ventilation is allowed, and acid when ventilation is kept uniform (fig. 162 to 165). In fig. 164 a temporary acid change occurs
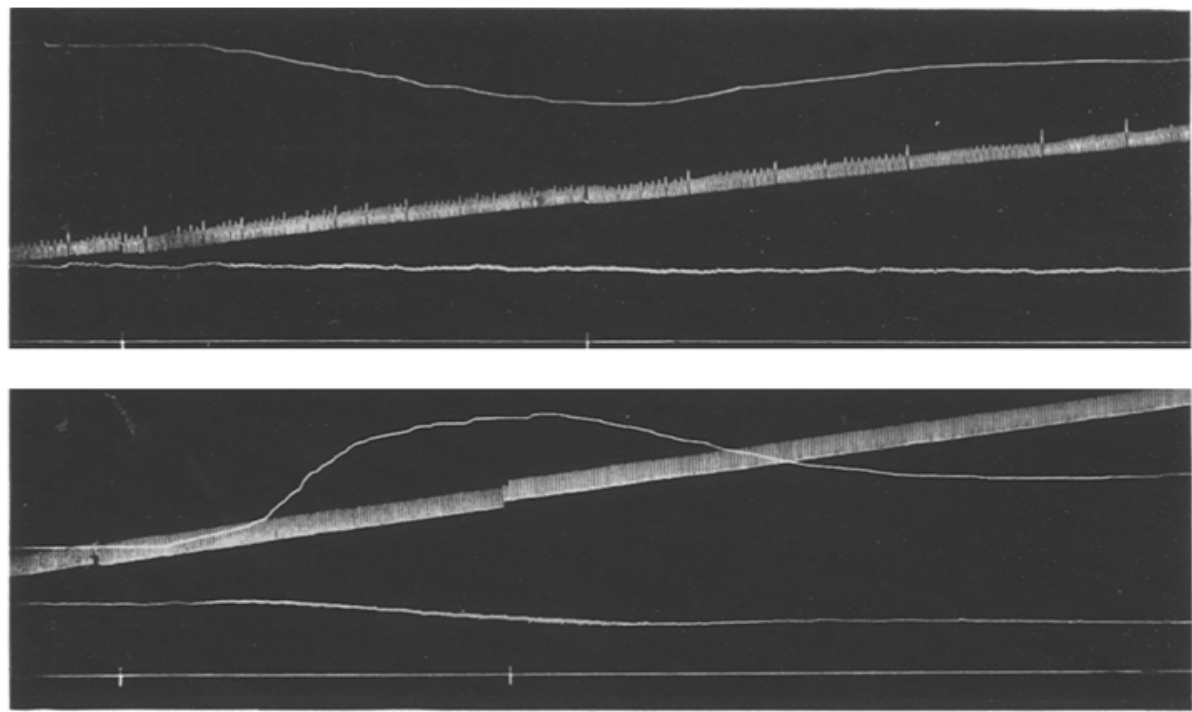

Fig. 166 and 167. Changes in $c_{H}$ recorded with a glass electrode resting lightly on the surface of the exposed. cerebral hemisphere of a dog. A gaseous mixture of low oxygen content is administered in both figures. In fig. 166 in which compensatory pulmonary ventilation is allowed the $c_{E}$ of the fluid in contact with the electrode decreases. In fig. 167 where such compensation is not allowed and in which the same gaseous mixture is administered by uniform artificial ventilation the $\mathrm{cH}$ of the fluid in contact with the electrode increases. It is concluded, therefore, that in the condition of this particular experiment the changes in acid metabolism of the brain cells exerts an acid effect upon its fluid environment. It should be remembered that the blood, which also is capable of modifying the $c_{\mathrm{H}}$ of tissue fluids, would necessarily turn more alkaline from reduction of hemoglobin alone. (BRAsGFIELD and HaNDs: Unpublished.)

despite a supernormal pulmonary ventilation indicating an extremely high rate of acid formation.

Electrodes placed on the surface of the brain show the same distinctly alkaline shift during oxygen deficiency when pulmonary ventilation increases (fig. 166) and an acid change when overventilation is prevented (fig. 167). Sometimes the electrodes indicate an initial alkaline swing which only later gives way to an acid shift. This may indicate that the effects exerted by the blood upon the electrode come on more quickly than those exerted by the brain cells. In other experiments the alkaline change is even more persistent, possibly indicating a compensatory increased volume flow of blood, for oxygen deficiency is known to markedly increase the volume flow of blood through the brain. Possibly later experiments will actually demonstrate that increased 
volume flow of blood can overcompensate for the cellular acid effects of hypooxia just as hyperventilation does. Even so the principles of the acidity theory of respiratory control would be unaffected.

A most interesting point of centrogenic and reflexogenic interaction is the opposite trends of hypercapnia and hypooxia in breathing. Hypercapnia tends to eliminate reflexogenic support of chemical origin in favor of complete centrogenic breathing of chemical origin. Hypooxia on the other hand tends to eliminate centrogenic breathing in favor of complete reflexogenic breathing. Of the two threats to a continuous flow of energy oxygen lack is the more dangerous. It is, therefore, well that a tough outlying structure, such as the carotid body would seem to be [Schmint and Comroe (171)] carries the brunt of respiratory control rather than the centers. Sensitive as the centers are to reduced oxidations, disaster might readily follow if they were subjected to the damaging effects required to produce stimulation. Hypercapnia on the other hand is much less injurious to tissue metabolism but its depressing effects upon chemical respiratory reflexes could on the other hand be most disastrious, had central chemical control of breathing not evolved. One need only picture an animal in violent combat to see the necessity of a central response to increased carbon dioxide. A sudden outpouring of carbon dioxide from the muscles and a nullification of peripheral chemical stimulation would put an end to breathing. Perhaps it might have been better so!?

\section{A Comparison of Hypooxic and Hypercapnic Hyperpnea.}

Oxygen deficiency and acid excess constitute the two primary physiological threats to a continuing supply of energy and there are reasons for believing that both are constantly at hand. It is their stimulating action which forces us to breathe for when removed by overventilation of the lungs breathing stops until the chemical stimulation returns. Assuming that oxygen deficiency or acid excess varies with changing physiological conditions, the type of breathing produced by a predominating action of either one or the other becomes a most interesting inquiry. Breathing, more or less characteristic for hypooxia and hypercapnia are illustrated in fig. 168 and 169. In general breathing during hypercapnia is slower and deeper than that of hypooxia and the chest and abdomen are compressed, rather than expanded, as in hypooxia.

These differences in hyperpnea now seem to have a simple explanation. For example the greater residual volume of the lungs during the expiratory phase in hypooxia is most probably a chemical reflex initiated in the chemoceptors. As we have already seen, the hyperpnea of hypooxia is entirely a reflexogenic phenomenon for the centrogenic breathing actually falls off to zero when chemoceptor activity is abolished by blocking Herings nerves in the vagotomized dog. Since the chemoceptors are connected with both respi- 
ratory half-centers some reason must be found for the selective tonic activity of the inspiratory half-center. This, we believe, is the preponderant impingement of chemoceptor signals, and consequently the preponderating after-

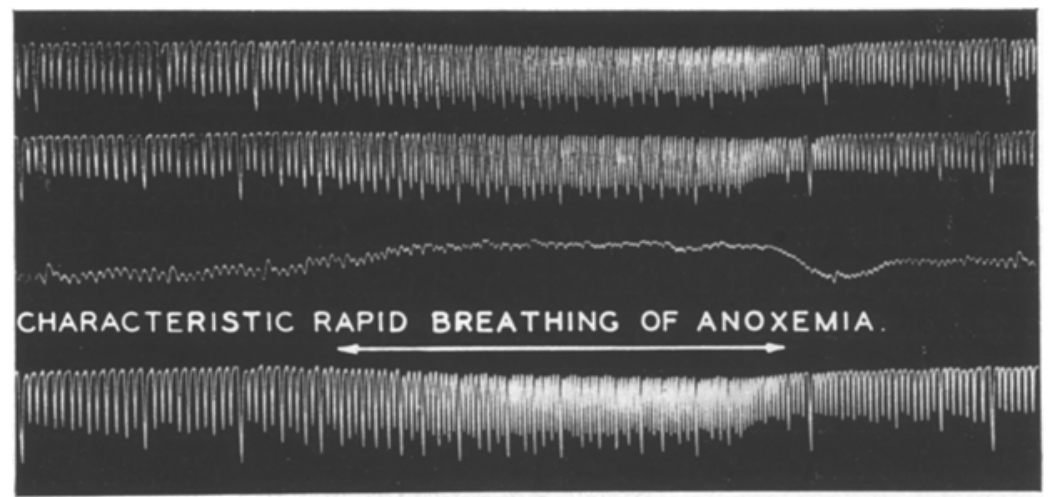

Fig. 168. Hypooxic hyperpnea produced by a temporary administration of a gaseous mixture low in oxygen content. The first and second tracings are respiratory excursions of the chest and abdomen recorded with encircling bands. The lower record is a spirometer tracing of pulmonary ventilation. Note a relatively small increase in the amplitude and a large increase in the frequency of breathing. The volume of the lungs at the end of expiration is greater during hyperpnea than during eupnea. Compare these results with those of hypercapnia in fig. 169. [Grselt and Moyfe: Quart. J. exper. Physiol. 24, 331 (1935).]

synaptic action in the inspiratory half-center. Fig. 170 showing the action of cyanide will illustrate, for the effects of cyanide are like those of oxygen deficiency. When hyperpnea is provoked by intravenous injection of cyanide,

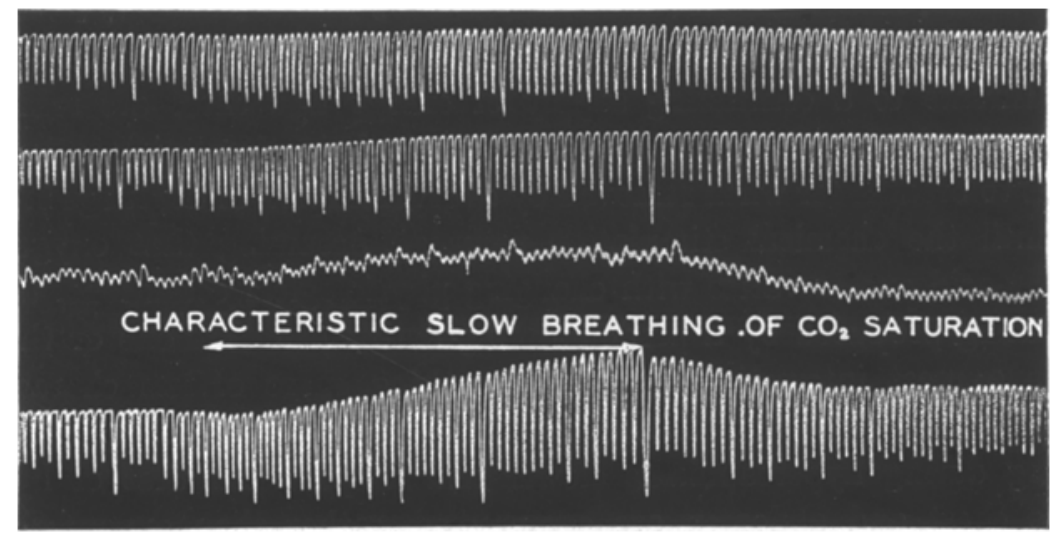

Fig. 169. Hypercapnie hyperpnea produced by a temporary administration of a gaseous mixture high in carbon dioxide content. See preceding figure for identification of tracings. Note the relatively small increase of rhythm and the large augmentation of amplitude of breathing. In contrast with the bypooxic hyperpnea of fig. 168 the chest and abdomen are seen to constrict during the expiratory phase of hyperpnea. [Grsel, and MoYkR: Quart. J. exper. Physiol. 94, 331 (1935).]

inspiratory activity, as reflected in the electrogram of the external intercostal muscle, is greatly strengthened and lengthened in record $B$. (The vagi are cut.) Expiratory activity, as indicated by the electrogram of the transverse sterni muscle, is shortened and only slightly strengthened. Later in 
hyperpnea (record C) inspiratory activity is strengthened still more and extended into the expiratory phase. This extension is no doubt a process of after-discharge resulting from the accumulative after action of the myriads of signals arising in the intensely stimulated chemoceptors. Expiratory activity has in the same time disappeared, no doubt from the reciprocal inhibiting action of the inspiratory after-discharge. This interpretation is strengthened by the return of the expiratory activity so soon as the inspiratory after-discharge disappears during recovery (see record $\mathrm{F}$ ).

Another striking example of reciprocal interaction of the inspiratory and expiratory halfcenters during long sustained effects of impaired oxidations is illustrated in fig. 171 . Initially cyanide produces powerful contractions of the expiratory muscles, as indicated"in the transterni response, which however diminish in intensity as inspiratory activity increases and inhibits the expiratory half

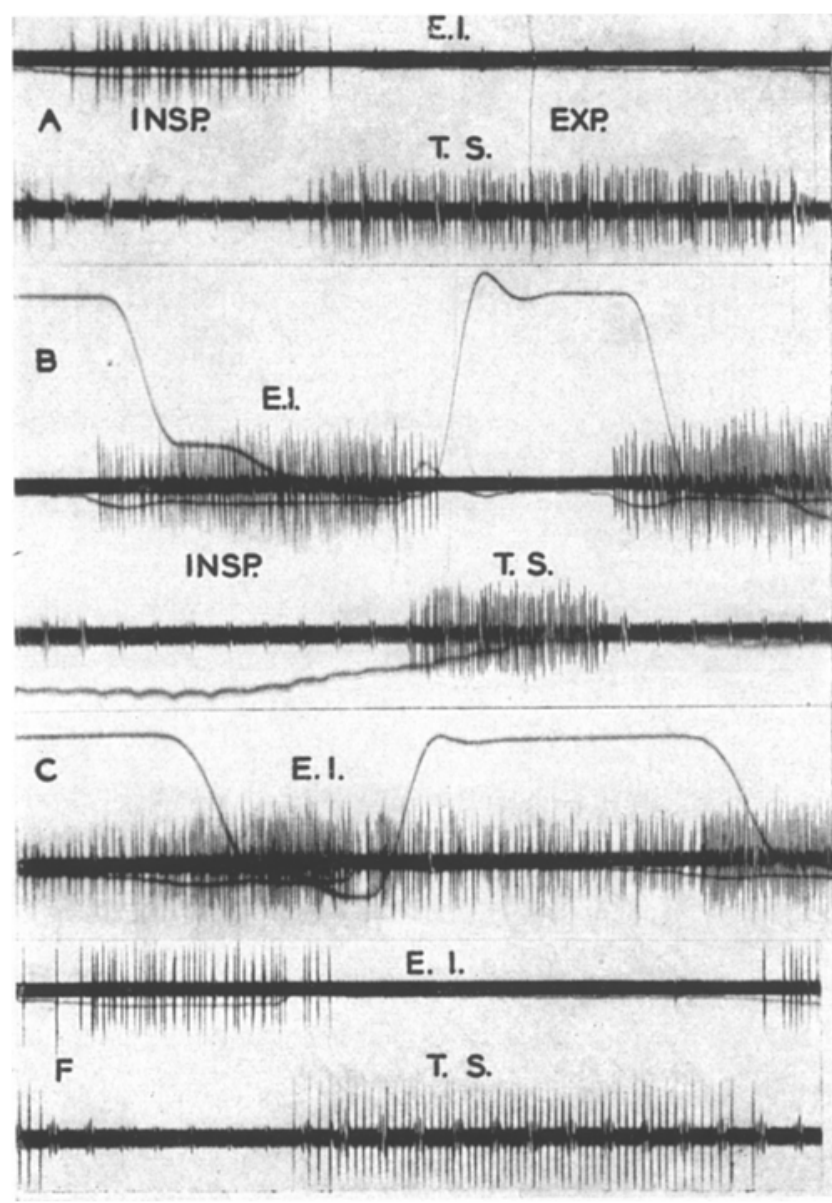

Fig. 170. Electrograms of inspiratory and expiratory muscles showing the interaction of these opposing groups during eupnea and hyperpnea produced by intravenous injection of cyanide, $\mathbb{E}$; I. External intercostal muscle and T, S. Transverse sterin müscle. Record A shows normal expiratory activity following upon normal inspiratory activity during euprea. In record $\mathrm{B}$ inspiratory activity is marked1y increased both in intensity and duration by cyanide. The effects in expiratory activity are less. As the action of cyanide continues the inspiratory discharge fails to end at the close of inspiration as shown by the powerful after-discharge extending throughout the plase of expiration. Duxing the period in which inspiratory after-discharges occur, expiratory activity is missing. This is explained by the reciprocating inhibition of expiratory activity exercised by the inspiratory afterdischarge, for as soon as this after-discharge disappears expiratory activity returns. See record F. [GESELI and WHITE: Amer. J. Physiol. 122, 48 (1938).] center. Increasing inspiratory activity is evidenced in two ways: by the electrogram of the internal intercostal muscle and by the increasing volume of the lungs during the expiratory phase (note downward trend of the tidal air record). 
In fig. 172 after-discharge develops in the inspiratory half-center without the slightest sign of stimulation on the expiratory side. But this record is of particular interest from another point of view, for it reveals the forces which are at work in the shifting of the respiratory phases. It will be seen that the first hyperpneic inspiratory discharges in record A cut off sharp and
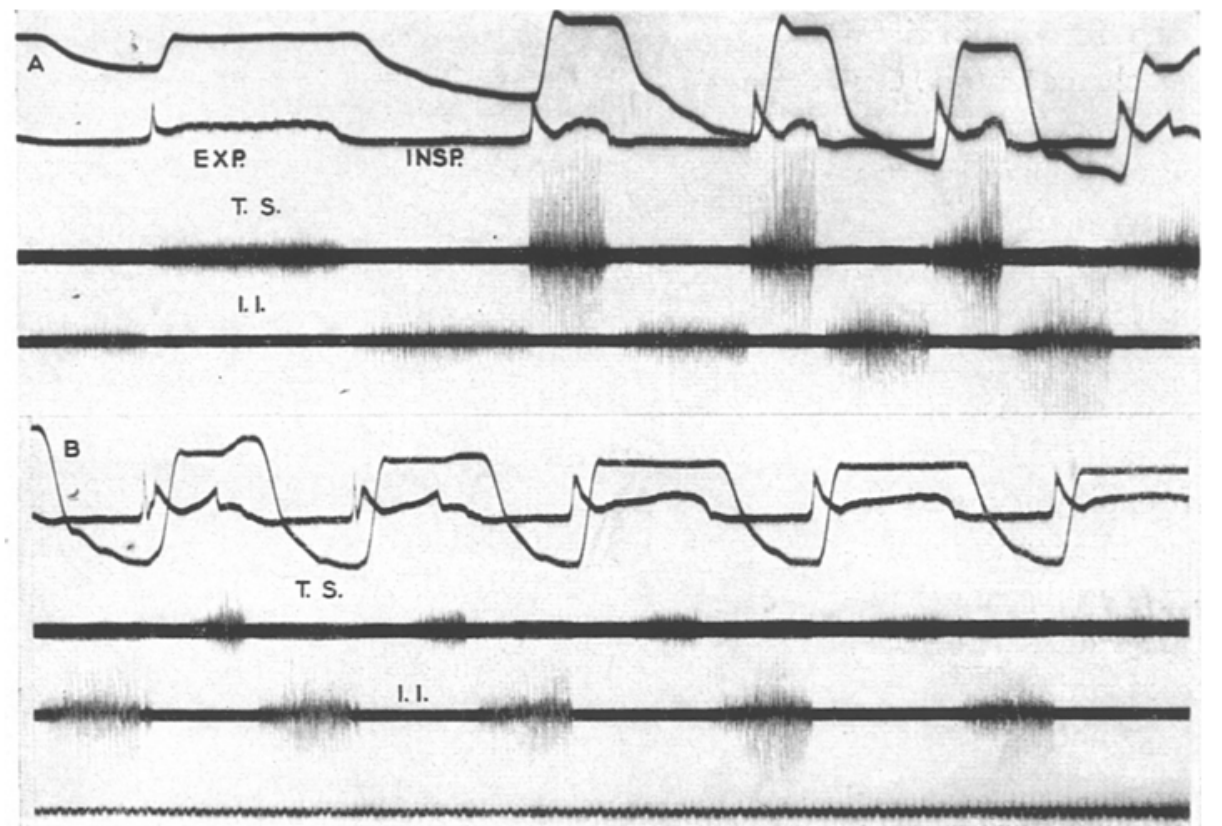

Fig. 171. Intravenous injection of cyanide produces a powerful stimulation of the expixatory muscles as indicated by the electrogram of the transverse sterni muscle. As hyperpnea continues inspiratory activity progressively augments and increases the volume of the lungs during the expiratory phase (see the falling expiratory level of the spirometer tracing). As this ocenrs, expiratory activity diminishes, suggesting that the expiratory half center is yielding to reciprocating inhibition coming from an increasingly active inspiratory center. [Geseld and WhITE: Amer. J. Physiol. 122, 48 (1938).]

clean at the end of inspiration. Momentarily they do the same in records $B$ and $\mathrm{C}$ but shortly after the end of inspiration a new set of discharges appear. Though separated by a short interval of time from the main inspiratory discharge they are no doubt the after-discharge of the normal inspiratory activity. If that is true, why do they not come on without the intervening silent period? We suggest that this most interesting phenomenon is due to a rebound effect caused by a sudden and normal weakening of the inspiratory discharge at the end of the inspiratory phase such as seen in record A. At that moment the inspiratory center suddenly withdraws its negative charges from the axon hillocks of the expiratory cells which are thereby stimulated. Their momentary activation in turn reciprocally inhibits the after-discharge of the inspiratory half-center thus producing the gap. Only after the rebound effect has disappeared is the inspiratory half-center free to after-discharge again. 
Why then is there no inspiratory after-discharge during hypercapnia? We have found none. This we believe is due to the fact that the hyperpnea of hypercapnia is a centrogenic activity, in which the signals initiated by carbon dioxide in the chemoceptors are counteracted by the action of carbon

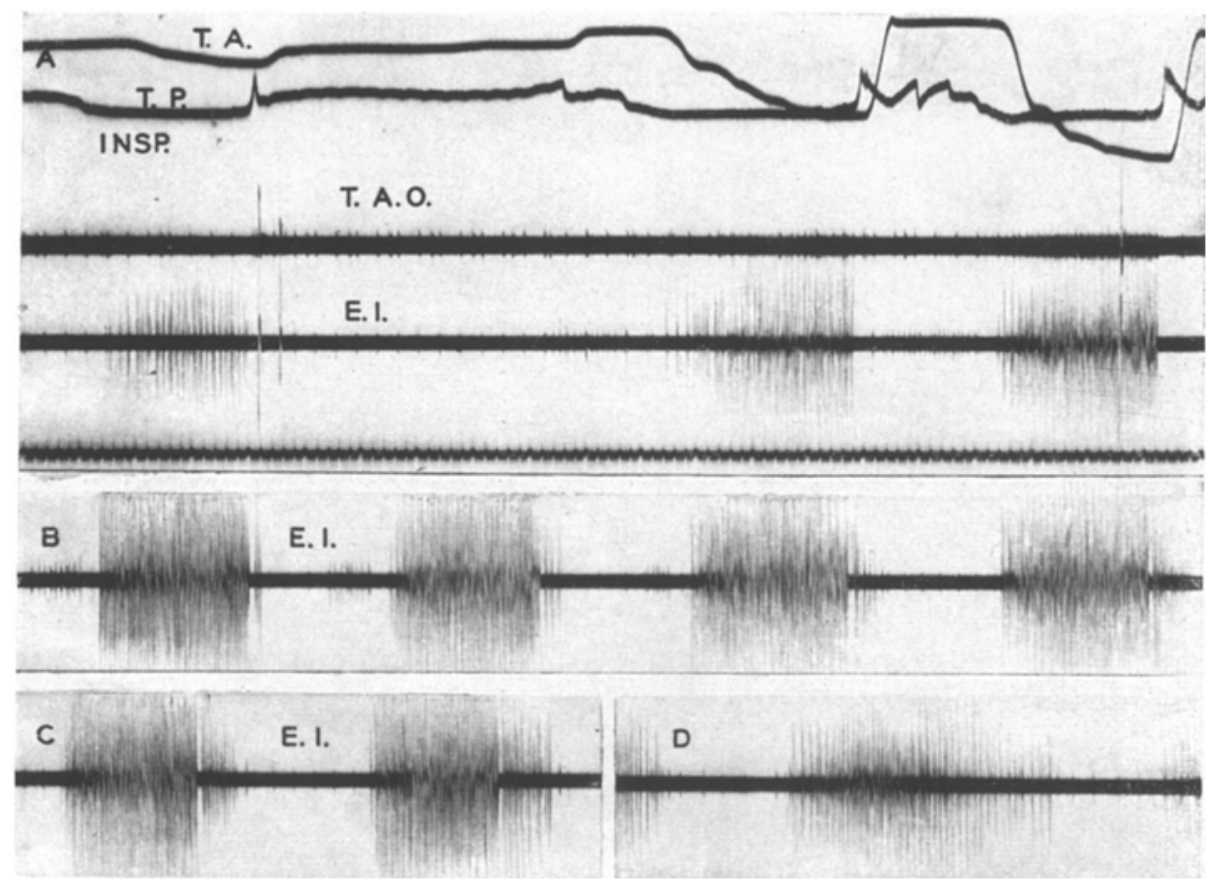

Fig. 172. Temporary inhibition of the inspiratory after-discharge. Cyanide increases the inspiratory activity of the external intercostal muscle but fails to increase the expiratory activity of the external abdominal muscle, (see record A). As hyperpnea progresses inspiratory after-discharges develop (see record A). As hyperpnea progresses inspiratory after-discharges develop (see records $B$ and $C$ ). Each after discharge is temporarily delayed. This is attributed to an hypothetically rebound effect produced by a sudden reduction of the inspiratory activity at the end of the inspiratory phase. This withdraws negative charges (inhibitory) from the axon hillock of the expiratory cells releasing them from their inhibition. The resulting activation of the expiratory half center momentarily inhibits the inspiratory after-discharge. In record $D$ in which the inspiratory after-discharge is virtually as strong as the original inspiratory discharge there can be no abrupt reduction of inspiratory activity at the end of the inspiratory phase and, therefore, rebound cannot develop. [Gesell and WhiTE: Amer. J. Physiol. 122, 48 (1938).]

dioxide at the center. After-synaptic action and its consequent after-discharge fail to develop.

The differences in frequency of breathing during hypooxia and hypercapnia are also related to the chemical conditions of the centers, particularly in relation to the vagal reflexes. The importance of the vagus nerves in frequency control has been discussed before in connection with the accelerated breathing produced by a combination of chemical and proprioceptive reflexes. For example, sulphide injected in the intact animal produces an enormous acceleration of breathing which is often missing when the vagi are blocked (see fig. 173). If we now bear in mind that carbon dioxide produces a chemical vagotomy by virtue of an increased $c_{\mathbf{H}}$ of the centers [GeserL and Moyer (79)]. 
and that hypooxic hyperpnea sensitizes the vagal reflexes by reducing the $c_{H}$ of the centers, the opposite effects of hypercapnia and hypooxia on fre-
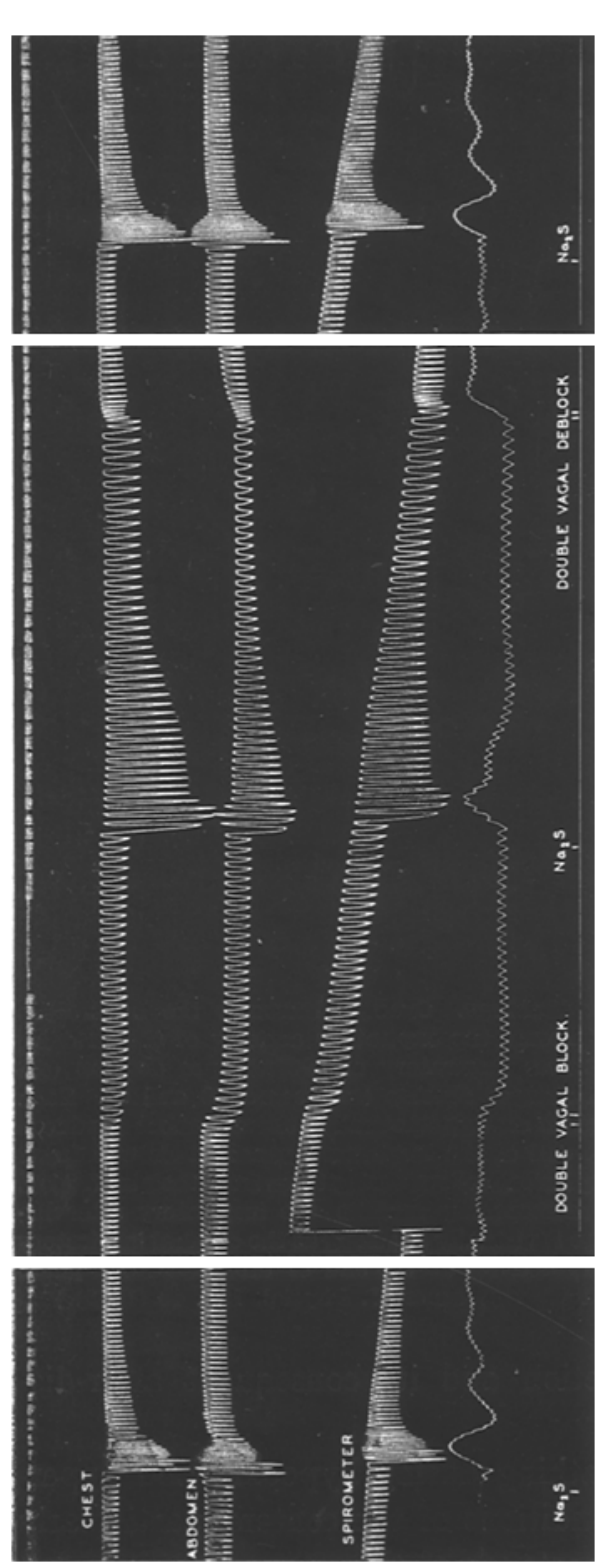

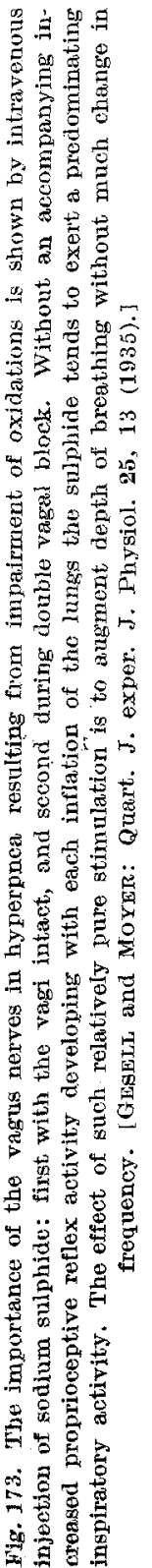
quency of breathing are readily understood.

One more factor explaining the frequency differences in breathing under these two conditions may be suggested and that is the greater expansion of the lungs during hypooxia. The increased residual discharge of the vagal stretch receptors might by itself decrease the frequency of breathing as suggested by Adrian (83) but it moust be remembered that a powerful inspiratory activity is added to an augmented expiratory activity, a combination which is capable of producing a high frequency of phase shift.

Fig. 174 will help to summarize this comparison of hypercapnia and hypooxia. For simplicity let us assume that hypercapnia abolishes all reflexes. The center then discharges by its own inherent rhythmic activity along the pattern lines ABC. But carbon dioxide increases the intensity of the discharge. This could accelerate or decrease the frequency of breathing as many experiments show. We have arbitrarily made the center discharge along the pattern lines A.D.C. Returning now to eupnea, by removal of hypercapnia we add the accelerating effects of the several proprioceptive reflex modifications of the original central discharge A.B.C. as indicated in the schema. The first modification is that of stretch reflex of the Golar endings. As a result of the intensification of the inspiratory activity the discharge comes 
to an earlier end. It grows and then expires along lines A.E.F. thus reducing the depth and increasing the frequency of breathing. Adding next the modifying effects of the hypothetical muscle spindle reflex the discharge now transpires along line A.G.H. which reduces the depth and increases the frequency of breathing still more. The vagal reflex modification produces the same
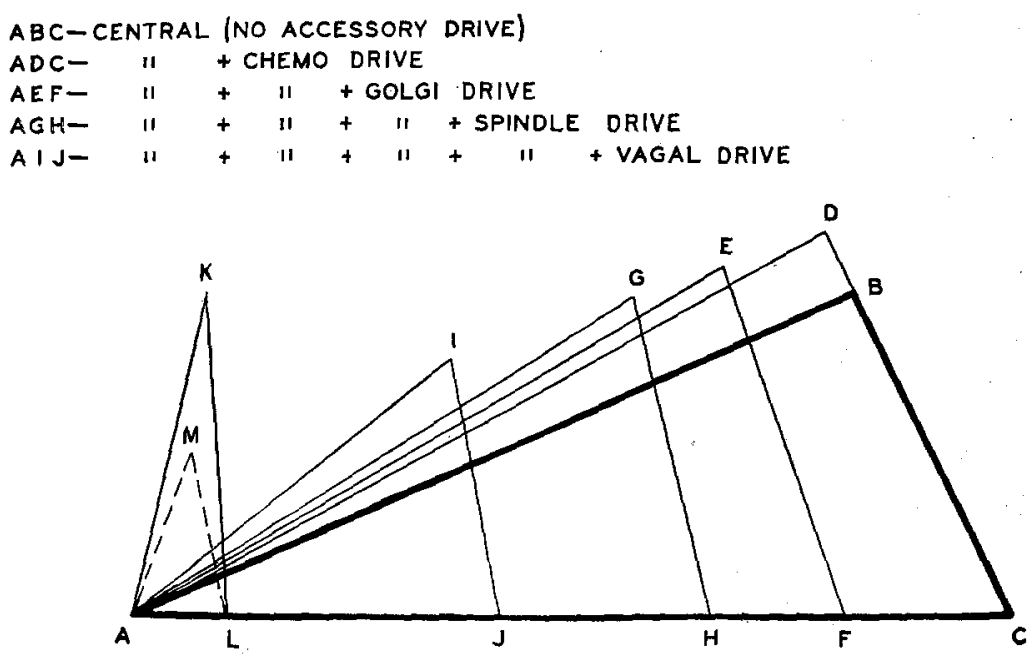

Fig. 174. A highly schematic diagram illustrating how the inherent discharge of the inspiratory center $A, B, C$ is modified by central and peripheral chemical stimulation and by the proprioceptive reflexes. Administration of carbon dioxide to a deafferented center increases the intensity of the central discharge and may either increase or decrease the rhythm of these discharges. That effect is schematically shown by triangle A, D, C. Removal of excess carbon dioxide and return of proprioceptive reflexes modifies the central discharge. Each proprioceptive reflex is believed to intensify and to cut the inspiratory discharge short thus decreasing the depth and increasing the frequency of breathing. These effects are shown for the GoLGI, the muscle spindle and the vagal stretch reflexes by triangles A, E, F, A. G. H., and A. I. J. Triangle A. I. J. represents normal eupneic breathing. Oxygen deficiency may modify eupnea by intensifying the discharge and by intensifying all modifying proprioceptive reflexes. If the intensification of the discharge predominates. breathing may be increased in depth as well as frequency. If the intensification of the modifying proprioceptive reflexes is excessive inspiration may be cut off prematurely. Breathing is accordingly shallow and exceedingly rapid. See triangles A. K. L. and K. M. L.

effects along limes A.I.J. which represents eupneic breathing. Hypooxia adds modifying effects to the eupneic discharge A.I.J. The increased activity of the chemoceptor endings tends to increase the depth of breathing. The increased intensification of the proprioceptive reflex modifications through alkalinization of the centers tends to cut inspiration shorter. Hyperoxic breathing thus becomes a resultant of these two effects, the balance of which determines the great variations which actually do occur during oxygen deficiency (see AML and AKL). 